九州大学学術情報リポジトリ

Kyushu University Institutional Repository

\title{
Sporen und Pollen der oberkretazeischen Hakobuchi Schichtengruppe, Hokkaido
}

Takahashi, Kiyoshi

Faculty of Science, Kyushu University

https://doi.org/10.5109/1543608

出版情報：九州大學理學部紀要：Series D, Geology. 14 (3)，pp.159-271，1964-01-20. Faculty of Science, Kyushu University バージョン:

権利関係 : 
Mem. Fac. Sci., Kyushu Univ., Ser. D, Geology, Vol. XIV, No. 3

pp. 159-271, tables 1-2, text-figs. 1-12, pls. 23-44, January 20, 1964

\title{
Sporen und Pollen der oberkretazeischen Hakobuchi- Schichtengruppe, Hokkaido*
}

\author{
Von \\ Kiyoshi TAKAHASHI
}

\section{Zusammenfassung}

Sechs Kohlenproben aus dem Kohlenflöz der unteren Hakobuchi-Schichtengruppe (Campan) von Hatsune-Sawa und zwei Proben aus den Feinsandsteinen der oberen Hakobuchi-Schichtengruppe (Maastricht) bei der Talspere von Ooyubari wurden pollenstratigraphisch und -paläontologisch untersucht. Ebenso wurde eine Kohlenprobe der Noborikawa-Schichten (Eozän), die die oberen Hakobuchi-Schichten diskordant überlagern, pollenanalytisch untersucht. Alle Sporomorphae wurden nach der binären Nomenklatur des Internationalen Codes benannt.

Die pollenstratigraphische Gliederung wurde hier einwandfrei festgelegt, nämlich 1) unteres Hakobuchi-Pollenbild, 2) oberes Hakobuchi-Pollenbild und 3) „Noborikawa“Pollenbild. Diese Pollenbilder wurden durch die Eigentümlichkeiten der Pollen- oder Sporengruppen und das Auftreten und Verschwinden der speziellen Arten bestimmt. Die meisten Sporen und Pollen kommen in allen Schichten vor, aber nur zweiundzwanzig spezielle Spezies treten in beiden Hakobuchi-Schichten gemeinsam auf und wenig davon in den Hakobuchi- und Noborikawa-Schichten gemeinsam. Man kann den größten Unterschied der Pflanzenwelt zwischen dem oberen Hakobuchi- und ,, Noborikawa“Pollenbild bemerken. Der Verfasser kann das untere und obere Hakobuchi-Pollenbild für den Typus des Pollenbildes von Campan und Maastricht in Nordjapan ansehen.

In Japan kann man die Normapolles-Pollengruppe, die im tiefen Alttertiär und in der Oberkreide von Europa und Ost-Amerika eine stratigraphisch sehr wichtige Pollengruppe ist, nicht finden. Das ist ein gemeinsames Phänomen in den Gebieten, die den Pazifik umgeben, z. B. in West-Amerika, West-Kanada, Japan, Neuseeland, Australien u. a. Man kann das als einen Unterschied der pflanzengeographischen Verbreitungsprovinz im tiefen Alttertiär und in der Oberkreide verstehen.

Viele neue Sporen- und Pollenformen wurden beschrieben und abgebildet. Sie wurden mit bereits bekannten ähnlichen Formen in der ganzen Welt verglichen.

\section{Inhalt}

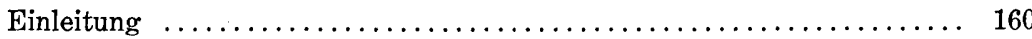

Stratigraphie der oberkretazeischen und alttertiären Formationen in der

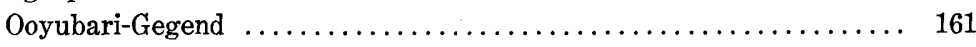

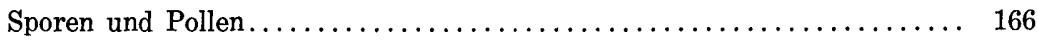

A) Sporen und Pollen der Hakobuchi-Schichtengruppe $\ldots \ldots \ldots \ldots 166$

B) Sporen und Pollen der Noborikawa-Schichten .............. 175

C) Oberkretazeische und alttertiäre Pollenbilder und ihr stratigraphisches Verhalten..................... 178

\footnotetext{
* Received June 29, 1963.

Diese Arbeit wurde am 24. Februar 1963 in der Jahrestagung der westiapanischen Zweigabteilung der japanischen geologischen Gesellschaft in Fukuoka vorläufig veröffentlicht.
} 
D) Geographische Verbreitung der wichtigen nachweisbaren Sporen

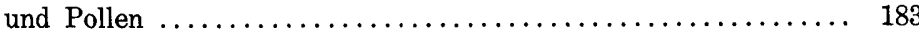

Beschreibung der Sporomorphae ....................... 190

A) Sporen und Pollen der Hakobuchi-Schichtengruppe ........ 190

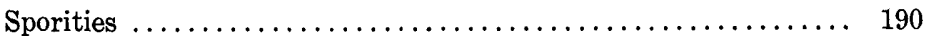

Triletes............................. 190

Zonales.............................. 212

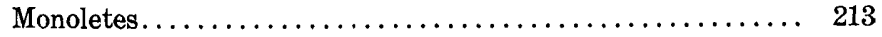

Incertae sedis . . . . . . . . . . . . . . . . . . . . . 221

Pollenites .................................... 221

Aletes $\ldots \ldots \ldots \ldots \ldots \ldots \ldots \ldots \ldots \ldots \ldots \ldots \ldots \ldots \ldots \ldots \ldots, 221$

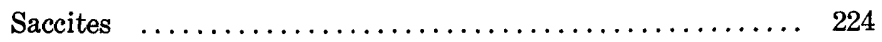

Poroses . . . . . . . . . . . . . . . . . . . . . . . . . . . . . 229

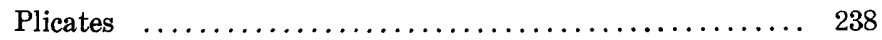

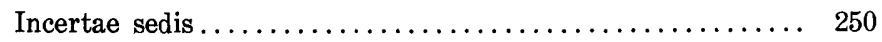

B) Sporen und Pollen der Noborikawa-Schichten............ 256

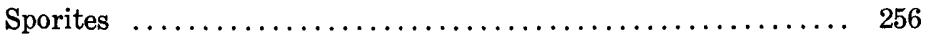

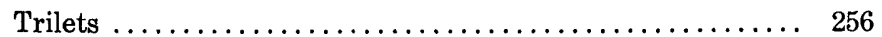

Pollenites. . . . . . . . . . . . . . . . . . . . . . . 257

Aletes $\ldots \ldots \ldots \ldots \ldots \ldots \ldots \ldots \ldots \ldots \ldots \ldots \ldots \ldots \ldots \ldots, 257$

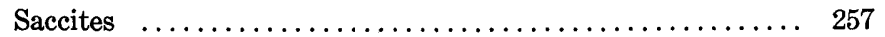

Poroses................................ 258

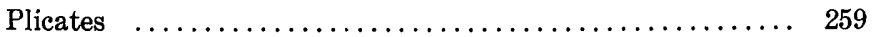

Jugates. ........................... 264

Literaturverzeichnis............................ 264

\section{Einleitung}

In Japan sind mesozoische Sporen und Pollen nur wenig untersucht worden. Bezüglich der mesozoischen Sporen und Pollen wurde bis heute nur die Arbeit von SATo (1961) veröffentlicht, trotzdem es über alttertiäre Sporen und Pollen viele Arbeiten gibt.

Der Verfasser hat hier die Sporen und Pollen der Hakobuchi-Schichtengruppe von Ooyubari (Campan und Maastricht) stratigraphisch und paläontologisch untersucht. Er hat dadurch das untere Hakobuchi-Pollenspektrum, als das typische Pollenbild des Campans und das obere Hakobuchi-Pollenspektrum als das typische Pollenbild des Maastrichts erkannt. Weiter hat er beide Pollenbilder mit einigen Pollenspektren der Oberkreide und des tiefen Paläogens in Kanada, USA, Australien, Neuseeland, Europa u. a. verglichen. In der Oberkreide und dem tiefen Paläogen von Europa und Ost-Amerika tritt die Normapolles-Pollengruppe als stratigraphisch wichtige Gruppe auf, aber in den Gebieten, die den Pazifik umgeben, kann man sie nicht bemerken. Das ist eine sehr wichtige Tatsache.

Viele Sporen- und Pollenformen aus der Hakobuchi-Schichtengruppe und den Noborikawa-Schichten wurden eingehend beschrieben und abgebildet. Sie wurden mit bereits bekannten ähnlichen Formen aus den verschiedenen Gegenden der ganzen Welt verglichen.

Bei dieser Arbeit möchte der Verfasser Herren Prof. Dr. Tatsuro MatsuMoto und Prof. Dr. Ryuzo TORIYAMA für ihre wertvolle Hinweise von ganzem 
Herzen danken. Ebenso muß er auch Herrn Prof. Dr. Hermann Weyland, Wuppertal-Elberfeld (Deutschland), für einige Hinweise und die Korrektur seines Schriftsatzes danken. Er ist auch Herrn Masaaki INouE an der Grube Ooyubari der Mitsubishi Kohlenbergwerk A. G. für ihre freundliche Hilfe beim Sammeln der Proben von ganzem Herzen dankbar.

\section{Stratigraphie der oberkretazeische und alttertiären Formationen in der Ooyubari-Gegend}

Zunächst werden die Hakobuchi-Schichtengruppe und die NoborikawaSchichten, deren Sporen und Pollen hier behandelt werden, kurz erläutert.

Die Hakobuchi-Schichtengruppe liegt in typischer Ausbildung bei der Talspere von Ooyubari. Der Verfasser hat dort zwei Sandsteine (Proben G
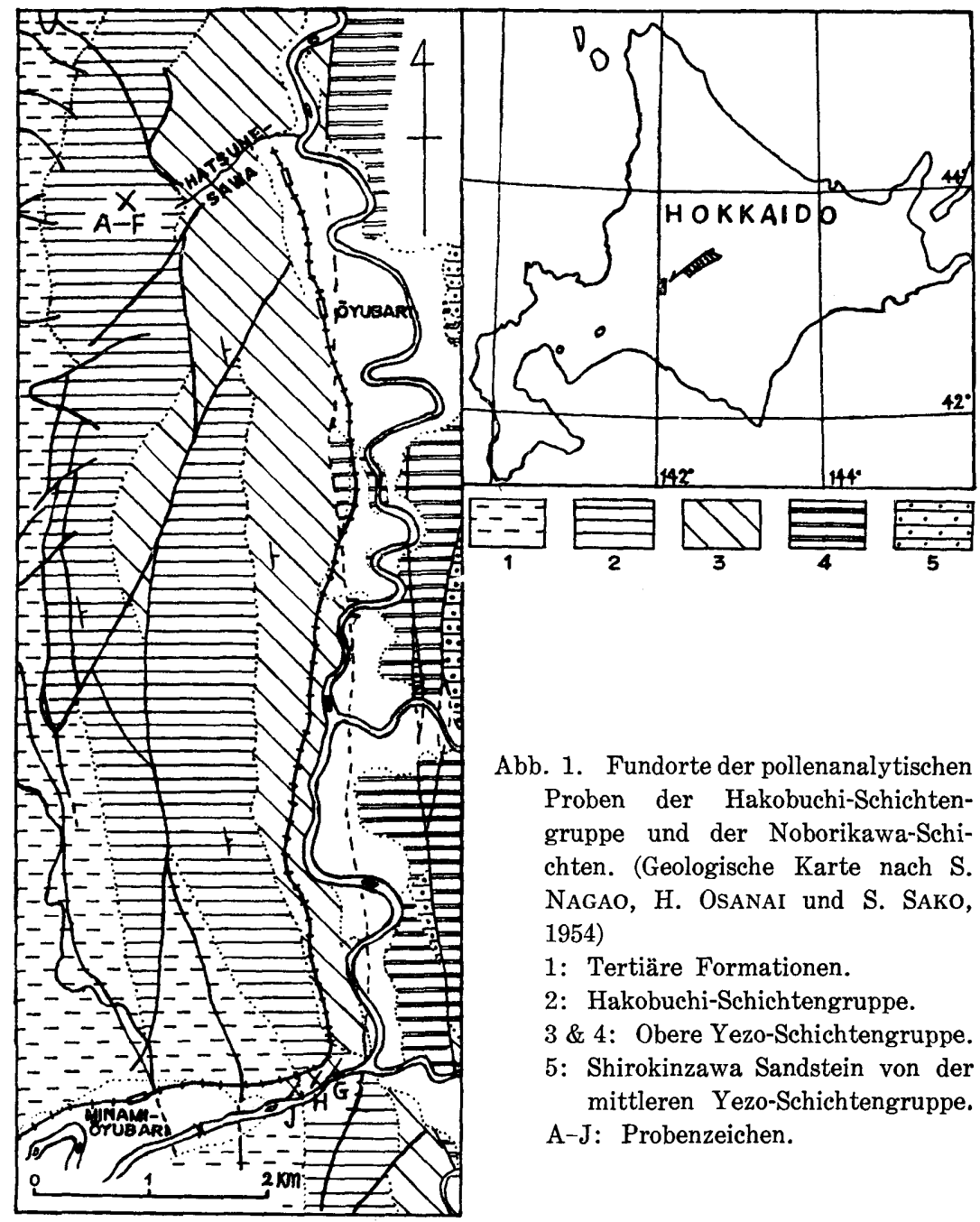

Abb. 1. Fundorte der pollenanalytischen Proben der Hakobuchi-Schichtengruppe und der Noborikawa-Schichten. (Geologische Karte nach S. NagaO, H. OSANAI und S. Sako, 1954)

1: Tertiäre Formationen.

2: Hakobuchi-Schichtengruppe.

3 \& 4: Obere Yezo-Schichtengruppe. 5: Shirokinzawa Sandstein von der mittleren Yezo-Schichtengruppe.

A-J: Probenzeichen. 
und $\mathrm{H}$ ) der oberen Hakobuchi-Schichtengruppe entnommen (s. Abb. 3). Er hat auch die Proben A-F aus dem Kohlenflöz der unteren Hakobuchi-Schichtengruppe von Hatsune-Sawa gesammelt.

Untersuchungen der Hakobuchi-Schichtengruppe und alttertiären Schichten im Ishikari-Kohlenfeld wurden schon früher von vielen Forschern durchgeführt. Die Hakobuchi-Schichtengruppe wurde von S. NAGAO, H. OsANAI und S. SAKo (1954) folgendermaßen eingeteilt:

Obere Hakobuchi-Schichtengruppe $250 \mathrm{~m}$

Sanushube Sandstein $(105 \mathrm{~m})$

Oberer Sandstein und Schieferton $(30 \mathrm{~m})$

Fukaushi Sandstein (35 m)

Fukaushi Konglomerat $(20 \mathrm{~m})$

Unterer Sandstein und Konglomerat $(60 \mathrm{~m})$

Untere Hakobuchi-Schichtengruppe $170 \mathrm{~m}$

Nilssonia-Schicht $(40 \mathrm{~m})$

Feinsandstein $(65 \mathrm{~m})$

Liparitischer Tuffstein $(25 \mathrm{~m})$

Sandstein mit dem konglomeratischen Teil $(40 \mathrm{~m})$

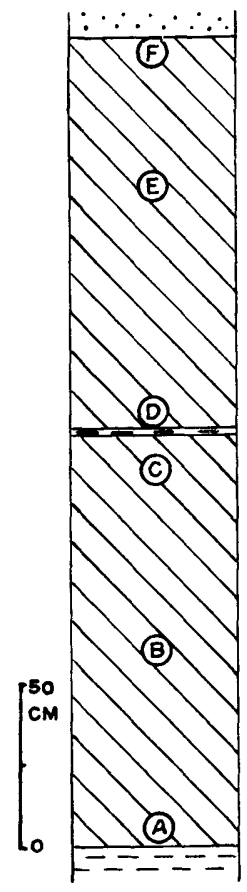

Abb. 2. Horizonte der Proben aus dem Kohlenflöz der unteren HakobuchiSchichtengruppe.

A-F: Probenzeichen.

Die Hakobuchi-Schichtengruppe überlagert die obere Yezo-Schichtengruppe konkordant In Hatsune-Sawa sind die unteren Teile der unteren HakobuchiSchichtengruppe marin und sie enthalten Muschelschalenreste von Inoceramus u. a. Die oberen Teile sind nicht-marin und enthalten das Kohlenflöz von etwa 
$2.4 \mathrm{~m}$ Mächtigkeit. Man findet die Nilssonia-Schicht etwa in den 7 oberen $\mathrm{m}$ dieses Kohlenflözes. S. ENDo (1925) hat die folgenden Spezies aus der NilssoniaSchicht beschrieben und abgebildet: Pteris frigida HEER, Asplenium dicksonianum HEER (?), Phyllites sp. (cf. Adiantum formosum HEER), Pecopteris torellii HeER (?), Glossozamites (?) imaii ENDo, Phyllites sp. (cf. Sphenozamites rogersianus FONTAINE), Cycadeoidea nipponica ENDO, Nilssonia cf. orientalis HEER, Nilssonia cf. johnstrupi HEER, Nilssonia serotina HEER, Nilssonia sp., Libocedrus sabiniana HEER, Sequoia heterophylla VELENovsky, Populus denticulata HEER, Populus arctica HEER (?), Rhamnites apiculatus LESQUEREUX und Protophyllum obovatum NEWBERRY.

In dem schwarzen Schieferton der Nilssonia-Schicht kann man nur sehr wenige Sporen und Pollen finden.

Die campanischen und maastrichtischen Inoceramus- und Ammoniten-Zonen wurden von T. MAтsumoto in der Hakobuchi-Schichtengruppe in der Hetonai Gegend festgelegt. In der Ooyubari-Gegend kann man keinen Inoceramus- und Ammoniten-Rest in der Hakobuchi-Schichtengruppe finden, abgesehen davon, daß man in unterestem Teil der Hakobuchi-Schichtengruppe nur wenigen Inoceramus-Rest finden kann. Der Hauptteil der unteren Hakobuchi-Schichtengruppe in der Hetonai Gegend gehört nach T. Matsumoto zu der Inoceramus

Tabelle 1. Biostratigraphische Zone der japanischen Oberkreide. (nach T. Matsumoto, 1959; ein Teil seiner Tabelle)

\begin{tabular}{|c|c|c|c|c|}
\hline \multirow[t]{2}{*}{$\begin{array}{l}\text { Ober- } \\
\text { Hetonai }\end{array}$} & \multirow[t]{2}{*}{$\mathrm{K}_{\beta} 6$} & $\begin{array}{l}\text { I. hetonaianus } \\
+ \text { I. (?) awajiensis }\end{array}$ & \multirow{2}{*}{$\begin{array}{l}\text { Pachydiscus subcompressus } \\
+ \text { Pach. (Neodesmoc.) } \\
\text { japonicus }\end{array}$} & \multirow[t]{2}{*}{ Maastricht } \\
\hline & & I. shikotanensis & & \\
\hline $\begin{array}{l}\text { Unter- } \\
\text { Hetonai }\end{array}$ & $\underset{\alpha}{\mathrm{K}} 6$ & I. schmidti & Canadoceras kossmati & \multirow{2}{*}{ Campan } \\
\hline $\begin{array}{l}\text { Infra- } \\
\text { Hetonai }\end{array}$ & $\underset{\gamma}{\mathbf{K}}$ & I. orientalis & A. (Neopachydiscus) & \\
\hline
\end{tabular}

schmidti-Zone und der Canadoceras kossmati-Zone. Die Hakobuchi-Schichtengruppe in der betreffenden Gegend ist von der Hakobuchi-Schichtengruppe in der Hetonai Gegend in der Litho- und Biofazies unterschieden, aber nach den bisherigen geologischen und stratigraphischen Arbeiten kommt es zu der Schlußfolgerung, daß die erstere zu dieser Zone gehöre.

Trigonia subovalis JIMBO var. minor YABE et NAGAO wurde aus der unteren Sandstein-Konglomerat-Schicht mitgeteilt. Das Fukaushi-Konglomerat ist sehr auffallend. Der Kies besteht aus Sandstein, Tonschiefer, Liparit u. a. Die Fukaushi-Sandstein-Schicht besteht im wesentlichen aus dem gaugrünen Feinsandstein. Es wurde Rhynchonella sp. (?) angegeben. Der Verfasser hat die Sandstein-Probe G aus dieser Schicht gesammelt. Die obere Sandstein-Schieferton-Schicht (Wechsellagerung) besteht aus hellgraugrünem mittel- bis feinkörnigem Sandstein und dunkelgrauem bis hellgrünem Schieferton. Die SanushubeSandstein-Schicht besteht aus dem graugrünen mittel- bis feinkörnigen Sandstein. 
In dieser Schicht haben S. NAGAO u. a. den Fischzahn, Lamna sp., gefunden. Der Verfasser hat die Sandstein-Probe H in dieser Sandstein-Schicht gesammelt. Nach T. Matsumoto entspricht die obere Hakobuchi-Schichtengruppe dem Ober-Hetonai (Maastricht). In der oberen Hakobuchi-Schichtengruppe in der betreffenden Gegend kann man die stratigraphisch wichtigen Fossilien noch

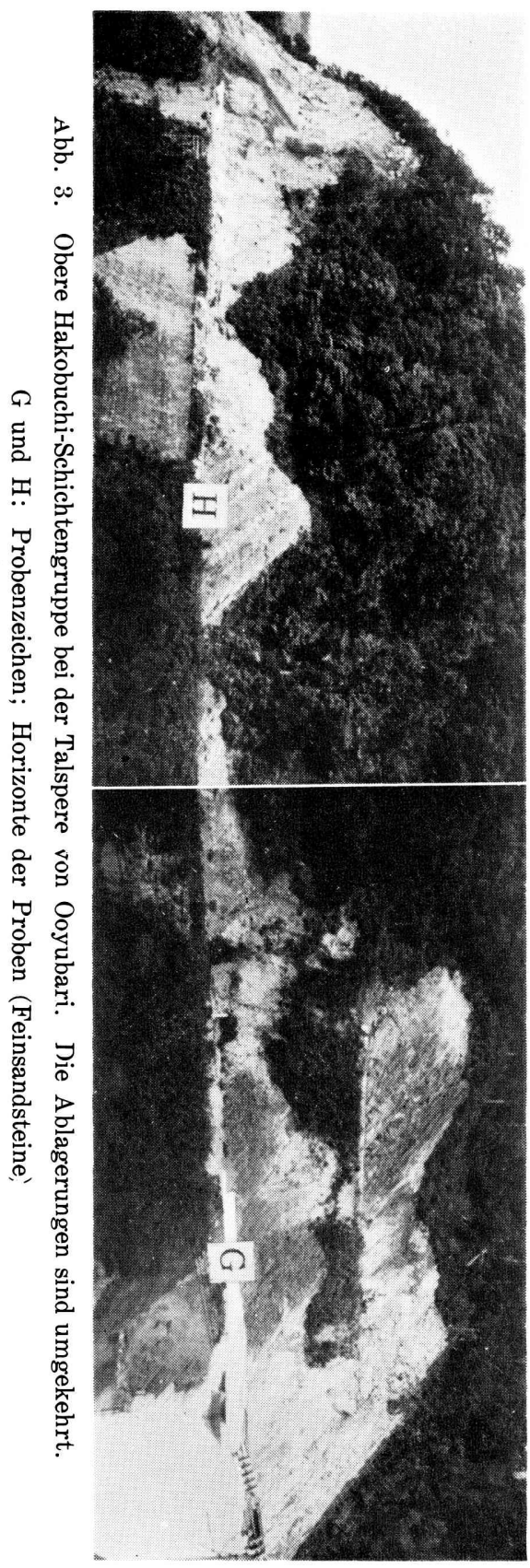


nicht finden, aber nach den bisherigen Berichten ist angenommen, daß diese Schichtengruppe jedoch zu der Inoceramus hetonaianus + Inoceramus (?) awajiensis Zone gehöre.

Die alttertiären Noborikawa-Schichten liegen auf der oberen HakobuchiSchichtengruppe bei der Talspere von Ooyubari parallel-diskordant auf und sind etwa $22 \mathrm{~m}$ mächtig. Sie bestehen aus Sandstein, Konglomerat, Schieferton und Kohlenfiözen (s. Abb. 4). Der Verfasser hat die Kohlenflöze I und J pollenanalytisch bearbeitet. Da aber das Kohlenflöz I gar keine Sporen und Pollen

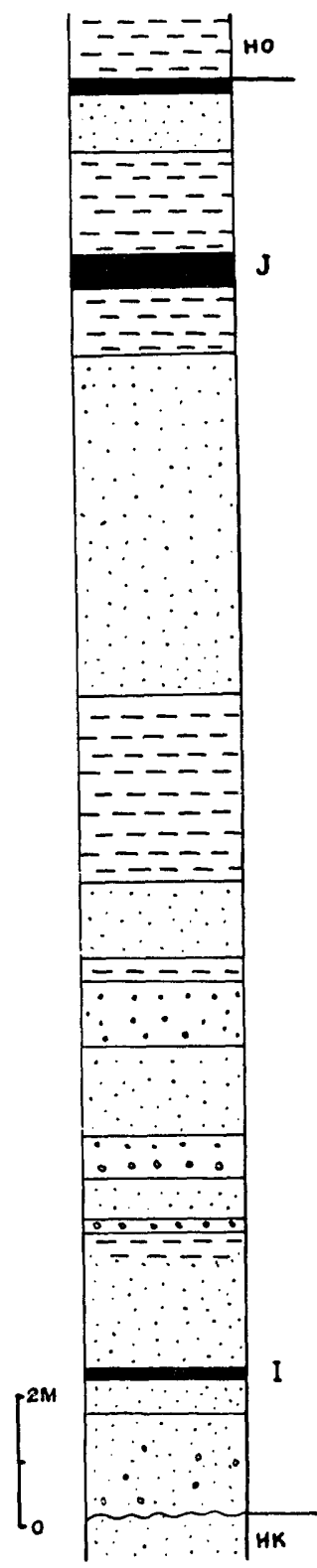

Abb. 4. Profil der Noborikawa-Schichten bei der Talspere von Ooyubari (nach INOUE \& MINAMI). HO: Horokabetsu-Schichten. HK: Hakobuchi-Schichtengruppe. $\mathrm{I}$ und J: Probenzeichen. 
enthält, muß er das Pollenspektrum des Kohlenflözes $J$ als vorläufigen Typus der Noborikawa-Schichten betrachten. Aus den Noborikawa-Schichten sind bisher nur Sequoia langsdorfii BRoNGN., Glyptostrobus europaeus HEER, Salix sp. u. a. bekannt.

Die palynologisch-stratigraphische Untersuchung der alttertiären Schichten im Ishikari-Kohlenfeld wird später ausführlich mitgeteilt werden.

\section{Sporen und Pollen}

Hier behandelt der Verfasser hauptsächlich die Sporomorphae der Hakobuchi-Schichtengruppe und beifügend die der Noborikawa-Schichten.

\section{A) Sporen und Pollen der Hakobuchi-Schichtengruppe.}

Bei der unteren Hakobuchi-Schichtengruppe handelt es sich um die Steinkohlen. Die Kohlenproben A-F wurden pollenanalytisch untersucht. Sie wurden zuerst im Eisenmörser zerkleinert und dann mit Schultzeschem Gemisch $\left(\mathrm{HNO}_{3}+\right.$ $\mathrm{KClO}_{3}$ ) und Fluorwasserstoffsäure $(\mathrm{HF})$ behandelt.

Die Sporen und Pollen aus dem Kohlenflöz der unteren Hakobuchi-Schichtengruppe findet man in der Tabelle 2. Von Sporen sind es 41 Spezies und von Pollen 62 Spezies. Von den Sporen- und Pollenarten sind die meisten Spezies neu, aber es gibt auch einige aus dem Ausland bekannte Spezies, z. B. Cicatricosisporites ef. dorogensis R. РОT. \& GELL., Laevigatosporites ovatus Wilson \& Webster und Inaperturopollenites magnus (R. Рот.) Thомson \& Pflug. Cicatricosisporites dorogensis R. Pot. \& Gell. (Schizaeaceae) wurde von R. РотоNiÉ und J. Gelletich (1933) aus den oberpaläozänen bis eozänen Schichten von Dorog, Ungarn, erstmalig beschrieben. Diese Spezies tritt im Alttertiär und Mesozoikum von Europa, Kanada, USA, Japan u. a. auf. Laevigatosporites ovatus WILsON \& WEBSTER (Polypodiaceae) ist eine amerikanische paläozäne Spezies. L. R. WILson und R. M. WeBsteR (1946) haben sie erstmalig aus den paläozänen Fort Union Schichten von Montana beschrieben. Sie tritt auch im Campan und Eozän von Westkanada auf. Inaperturopollenites magnus (R. Pot.) Thomson \& Pflug (Pseudotsuga, Larix u. a.) wurde von R. Potonié (1934) erstmalig aus den eozänen Schichten des Geiseltales (Deutschland) beschrieben.

Von den Sporenarten sind Gleicheniidites marginatus n. sp. (Gleicheniaceae), Stereisporites limbatus n. sp. (Sphagnum), Stereisporites pseurlostereoides n. sp. (Sphagnum), Punctatisporites punctulatus n. sp., Apiculatisporis inouei n. sp. (Osmundaceae), Baculatisporites papillosus n. sp. (Osmundaceae), Baculatisporites validus n. sp. (Osmundaceae), Laevigatosporites prominens n. sp. (Polypodiaceae), Laevigatosporites probatus n. sp. (Polypodiaceae), Laevigatosporites dehiscens TAKAHASHI (Polypodiaceae), Laevigatosporites senonicus n. sp. (Polypodiaceae) und Polypodiisporites repandus n. sp. (Polypodiaceae) in der Frequenz des Auftretens überwiegend. Stereisporites grossus n. sp. (Sphagnum) tritt nur im oberen Teil des Kohlenflözes der unteren Hakobuchi-Schichtengruppe auf. 
Tabelle 2. Sporen- und Pollenliste der unteren Hakobuchi-Schichtengruppe von Hatsune-Sawa.

Sporen und Pollen

Proben

Cicatricosisporites ef. dorogensis R. POT. \& GELL.

Gleicheniidites marginatus $\mathrm{n}$. sp.

Concavisporites macellus $\mathrm{n}$. sp.

Concavisporites sp. a

? Divisisporites sp.

Verrucosisporites permirus n. sp.

Stereisporites limbatus n. sp.

Stereisporites grossus n. sp.

Stereisporites pseudostereoides $\mathrm{n}$. sp.

Stereisporites ? tenuiculus n. sp.

Stereisporites sp.

Laevigatisporites magnus $\mathrm{n}$. sp.

Laevigatisporites hokkaidoensis $\mathrm{n}$. sp.

Punctatisporites punctulatus n. sp.

Punctatisporites hatsunesawaensis $\mathrm{n}$. $\mathrm{sp}$.

Punctatisporites sp.

Dictyophyllidites divergens (SATO) n. comb.

Corrugatisporites sp.

Rugulatisporites salebrosus n. sp.

Rugulatisporites parvirugulatus n. sp.

Microreticulatisporites sp.

? Microreticulatisporites sp.

Apiculatisporis inouei n. sp.

Apiculatisporis micracanthus n. sp.

Baculatisporites papillosus n. $\mathrm{sp}$.

Baculatisporites validus $\mathrm{n}$. sp.

Triplanosporites sinuatus $\mathrm{n}$. $\mathrm{sp}$.

Biretisporites ? minus n. sp.

Laevigatosporites prominens $\mathrm{n}$. $\mathrm{sp}$.

Laevigatosporites probatus n. sp.

Laevigatosporites dehiscens TAKAHASHI

Laevigatosporites senonicus $\mathrm{n}$. $\mathrm{sp}$.

Laevigatosporites ovatus WILSON \& WEBSTER

Laevigatosporites sp.

Polypodiisporites repandus n. $\mathrm{sp}$.

Polypodiisporites invisus n. sp.

Verrucatosporites sp. a

Undulatosporites rugulatus $\mathrm{n}$. $\mathrm{sp}$.

Cicatricososporites ? ellipsoideus n. sp.

? Cicatricososporites sp.

Schizosporis sp.

Weylandipollis retiformis $\mathrm{n}$. sp.

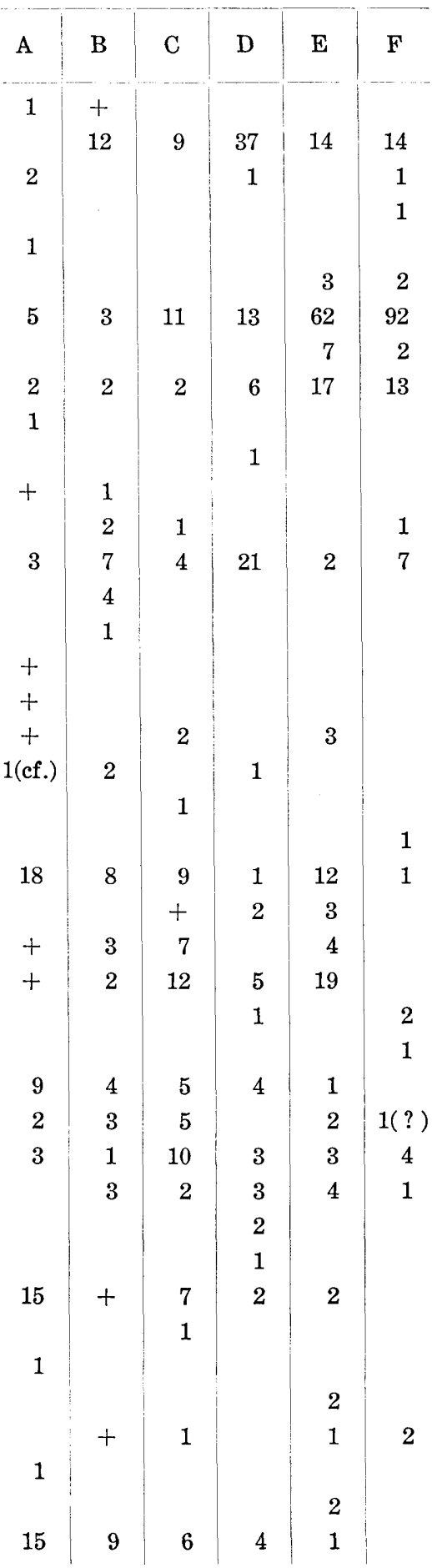




\begin{tabular}{|c|c|c|c|c|c|c|}
\hline Sporen und Pollen $\quad$ Proben & A & B & $\mathrm{C}$ & $\mathrm{D}$ & $\mathrm{E}$ & F \\
\hline Monocolpopollenites shiyuparoensis $\mathrm{n} . \mathrm{sp}$. & & 8 & 15 & 2 & & \\
\hline Monocolpopollenites kyushuensis TAKAHASHI & 1 & & 1 (cf.) & & 1 & \\
\hline Monocolpopollenites pfugii TAKAHASHI & & 1 (cf.) & 2 & 2 & & $4(\mathrm{cf})$. \\
\hline Monocolpopollenites asymmetricus $\mathrm{n} . \mathrm{sp}$. & & & & & 1 & \\
\hline Monocolpopollenites sp. a & 1 & & & & & \\
\hline Monocolpopollenites $\mathrm{sp} . \mathrm{b}$ & 1 & & & & & \\
\hline Monocolpopollenites sp. c & & & & 1 & & \\
\hline Inaperturopollenites pseudodubius TAKAHASHI & 67 & 43 & 17 & 27 & 15 & 10 \\
\hline $\begin{array}{l}\text { Inaperturopollenites magnus (R. Рот.) THомson } \\
\text { \& PFLUG }\end{array}$ & + & & & & & \\
\hline Pityosporites aliformis n. sp. & 3 & + & 1 & & 1 & 2 \\
\hline Pityosporites microaliformis n. sp. & + & & & 2 & & \\
\hline Pityosporites sp. & 1 & & & & & \\
\hline Piceaepollenites saccellus n. sp. & + & 2 & & & & \\
\hline Podocarpidites sp. a & 1 & & & & & \\
\hline Podocarpidites sp. $\mathrm{b}$ & & & 1 & & & \\
\hline Phyllocladidites brachypterus n. sp. & + & 1 & & & & \\
\hline Phyllocladidites mirandus n. sp. & & & 1 & 1 & & \\
\hline Alnipollenites eminens (TAKAHASHI) $\mathrm{n}$. comb. & 3 & 49 & 36 & 20 & 4 & 10 \\
\hline Momipites constatus (TAKAHASHI) n. comb. & & 1 & & & 1 & 2 \\
\hline Triporopollenites festatus TAKAHASHI & & & 1 & & 1 (cf.) & \\
\hline Triporopollenites kasuyaensis TAKAHASHI & & & & & & 1 \\
\hline Subtriporopollenites kyushuensis TAKAHASHI & & & 1 & & & \\
\hline Betulaepollenites normalis $\mathrm{n} . \mathrm{sp}$. & 3 & & + & 5 & 2 & 3 \\
\hline Graminidites microapertus n. sp. & 1 & & & 1 & & \\
\hline Tricolpopollenites umiensis TAKAHASHI & 1 & & & & & \\
\hline Tricolpopollenites cf. ditis TAKAHASHI & & & & 1 & & \\
\hline $\begin{array}{l}\text { Tricolpopollenites meinohamensis TAKAHASHI } \\
\text { subsp. rotundus TAKAHASHI }\end{array}$ & 3 & 1 & 1 & & & \\
\hline Tricolpopollenites cf. weylandii TAKAHASHI & 1 & & & & & \\
\hline Tricolpopollenites cf. microreticulatus TAKAHASHI & 3 & 3 & & & & \\
\hline Tricolpopollenites cf. subasper TAKAHASHI & & & 3 & & & \\
\hline Tricolpopollenites ef. striatellus TAKAHASH & & & & & & 1 \\
\hline Tricolpopollenites minutiretiformis n. sp. & 15 & 17 & 17 & 22 & 7 & 19 \\
\hline Tricolpopollenites conicus $\mathrm{n} . \mathrm{sp}$. & 1 & & 1 & & & \\
\hline Tricolpopollenites sp. a & 1 & & 1 & & & \\
\hline Tricolpopollenites sp. b & 1 & & & & & \\
\hline Tricolpopollenites sp. c & & & 1 & & & \\
\hline Tricolpopollenites sp. d & & & & 1 & & \\
\hline Tricolpopollenites sp. $\mathrm{g}$ & & 1 & & & & \\
\hline Tricolpopollenites sp. $\mathrm{h}$ & & & & 1 & & \\
\hline Tricolpopollenites sp. i & 1 & & & & & \\
\hline Tricolpopollenites $\mathrm{sp} . \mathrm{j}$ & & & & & 1 & \\
\hline Rhoipites ? minutireticulatus n. sp. & & 3 & 2 & 2 & & \\
\hline Tricolporopollenites minor TAKAHASHI & & 1 & & 1 & 1 (cf.) & \\
\hline
\end{tabular}




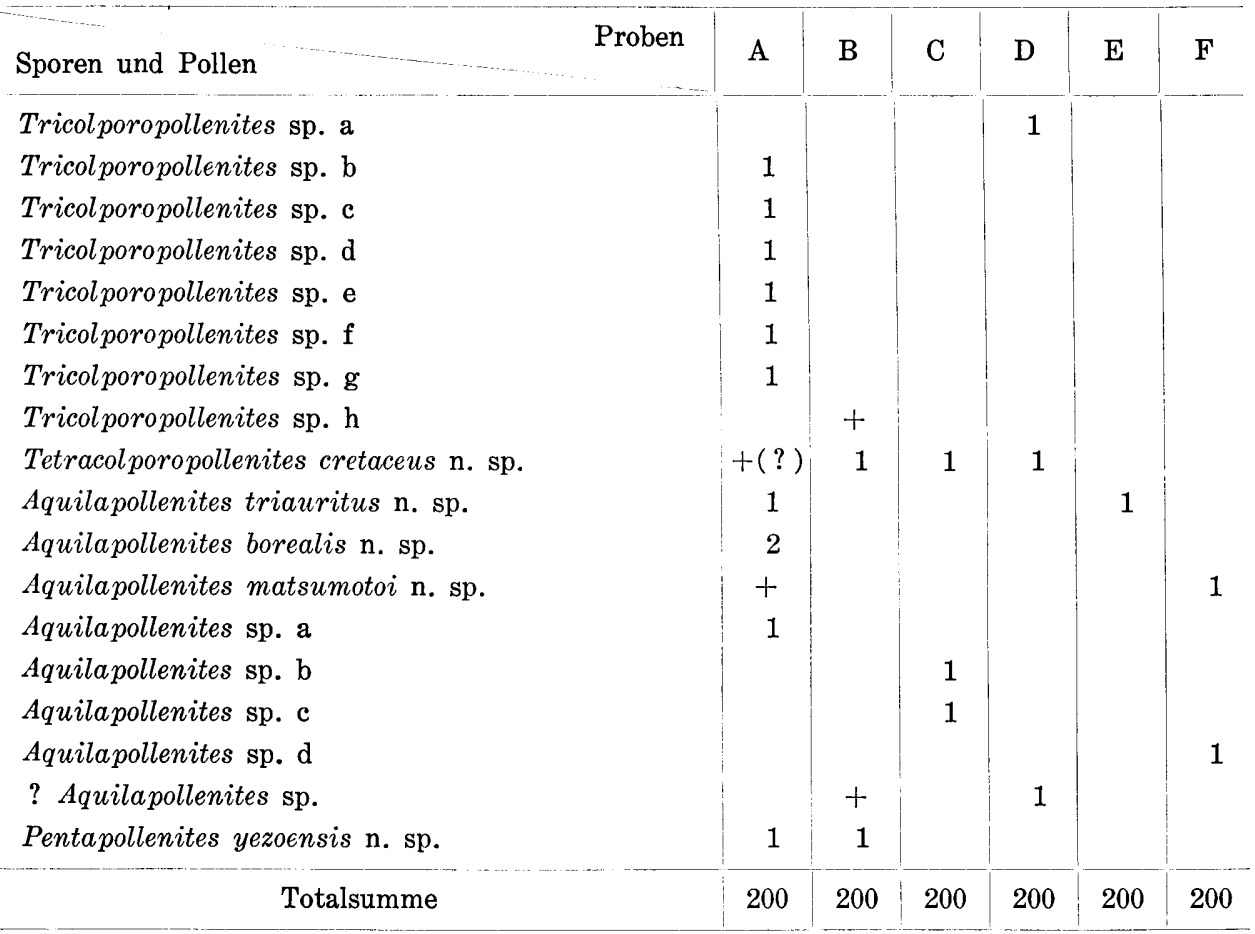

+ : Diese Bezeichnung zeigt das Vorhandensein des Pollens, der bei der Zählung nicht aufgetreten ist.

(Proben A-F siehe Abbildung 2)

Laevigatosporites dehiscens TAKAHASHI wurde vom Verfasser (1961) aus dem Alttertiär und Untermiozän von Westjapan beschrieben. Diese Form kommt hier auch verhältnismäßig häufig vor. Die Gattung Schizosporis wurde zuerst von I. C. Cookson und M. E. Dettmann (1959) aus den ober- bis unterkretazeischen Schichten (Apt-Cenoman) von Südaustralien beschrieben. S. A. J. Pocock (1962) hat einige Spezies von Schizosporis aus den jurassischen bis kretazeischen Schichten von Westkanada beschrieben. Zur Zeit ist Schizosporis nur in dem Oberjura und der Kreide von Westkanada, Nordjapan und Südaustralien bekannt.

Von den Pollenspezies sind Weylandipollis retiformis n. gen., n. sp., Monocolpopollenites shiyuparoensis n. sp., Inaperturopollenites pseudodubius TAKAHASHI (Taxodiaceae, Cupressaceae u. a.), Alnipollenites eminens (TAKAHASHI) (Alnus), Betulaepollenites normalis n. sp. (Betula-Typus), Tricolpopollenites minutiretiformis n. sp. (Salix ?, Platanus ?) u. a. häufig.

Folgende Spezies, die vom Verfasser $(1957,1961,1962)$ aus dem Alttertiär und Miozän von Westjapan beschrieben wurden, kann man in der HakobuchiSchichtengruppe bemerken: Monocolpopollenites kyushuensis TAKAHASHI (Palmae, Ginkgoinae), Inaperturopollenites pseudodubius TAKAHASHI, Alnipollenites eminens (TAKAHASHI), Momipites constatus (TAKAHASHI) (Betulaceae), Triporopollenites festatus TAKAHASHI (Betulaceae), Triporopollenites kasuyaensis 


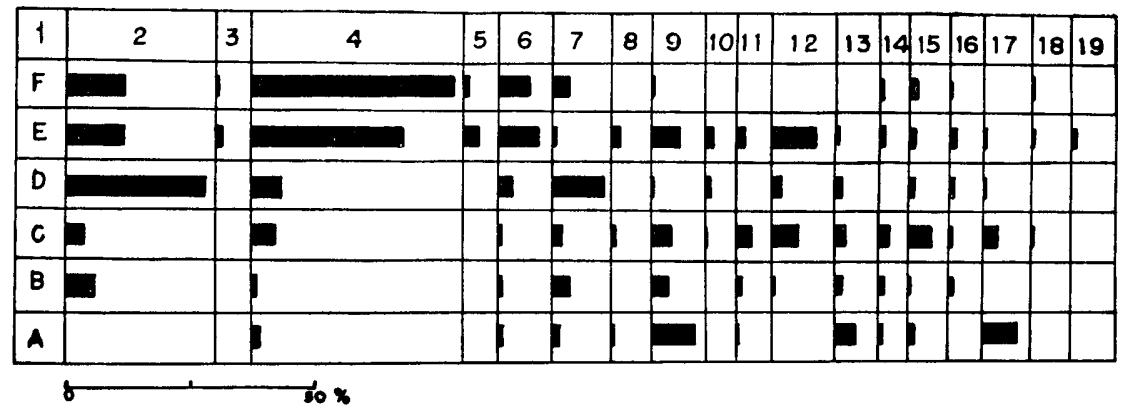

Abb. 5. Sporendiagramm der unteren Hakobuchi-Schichtengruppe.

1: Proben.

2: Gleicheniidites marginatus n. sp.

3: Verrucosisporites permirus n. sp.

4: Stereisporites limbatus n. sp.

5: Stereisporites grossus n. sp.

6: Stereisporites pseudostereoides n. sp.

7: Punctatisporites punctulatus n. sp.

8: Rugulatisporites salebrosus n. sp.

9: Apiculatisporis inouei n. sp.

10: Apiculatisporis micracanthus n. sp.

11: Baculatisporites papillosus n. sp.

12: Baculatisporites validus $\mathrm{n}$. $\mathrm{sp}$.

13: Laevigatosporites prominens $\mathrm{n}$. sp.

14: Laevigatosporites probatus $\mathrm{n}$. $\mathrm{sp}$.

15: Laevigatosporites dehiscens TAKAHASHI

16: Laevigatosporites senonicus $\mathrm{n}$. $\mathrm{sp}$.

17: Polypodiisporites repandus n. sp.

18: Cicatricososporites ? ellipsoideus n. sp.

19: Schizosporis sp.

TAKAHASHI (Betulaceae ?), Subtriporopollenites kyushuensis TAKAHASHI (Carya ?), Tricolpopollenites umiensis TAKAHASHI (Cupuliferae), Tricolpopollenites cf. ditis TaKahashi (Cupuliferae), Tricolpopollenites meinohamensis TAKaHashi subsp. rotundus TAKaHAshi, Tricolpopollenites cf. weylandii Takahashi, Tricolpopollenites cf. microreticulatus TAKAHASH (Salix, Platanus), Tricolpopollenites cf. subasper TAKAHASHI, Tricolpopollenites cf. striatellus TAKAHASHI und Tricolporopollenites minor TAKAHASHI.

Von diesen treten die meisten Spezies außer Inaperturopollenites pseudodubius TAKAHASHI und Alnipollenites eminens (TAKAHASHI) sehr selten auf (s. Tabelle 2).

Der Verfasser hat hier die neue Formgattung Weylandipollis vorgeschlagen. Die merkwürdige Form, Phyllocladidites, wurde bisher nur aus dem Alttertiär von Südaustralien, der Kerguelen Insel u. a. mitgeteilt. Die eigentümliche Form, Aquilapollenites, wurde zuerst von G. E. Rouse (1957) vorgeschlagen. Später hat J. W. Funk houser (1961) sie emendiert beschrieben. Diese Gattung ist bisher nur im Campan von Westkanada, im Campan bis Paläozän von Wyoming (USA) und in der Oberkreide vom Vilyusk-Becken (Sibirien) bekannt. In 


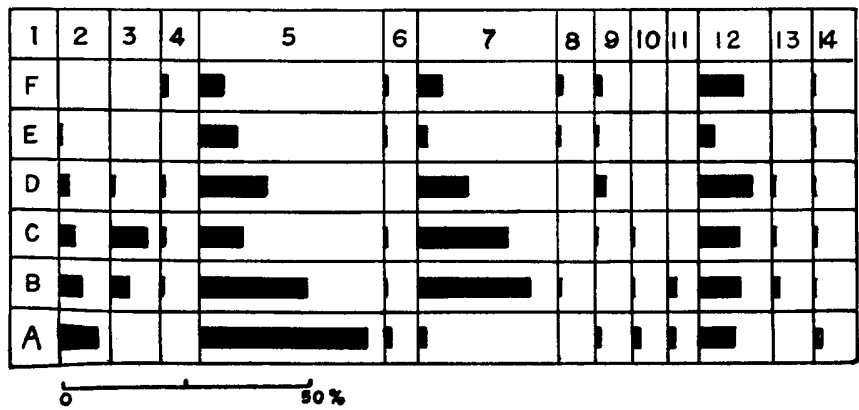

Abb. 6. Pollendiagramm der unteren Hakobuchi-Schichtengruppe.

1: Proben.

2: Weylandipollis retiformis $\mathrm{n}$. $\mathrm{sp}$.

3: Monocolpopollenites shiyuparoensis $\mathrm{n}$. sp.

4: Monocolpopollenites pflugii TAKaHASH

5: Inaperturopollenites pseudodubius TAKAHASHI

6: Pityosporites aliformis $\mathrm{n}$. sp.

7: Alnipollenites eminens (TAKAHASHI)

8: Momipites constatus (TAKAHASHI)

9: Betulaepollenites normalis $\mathrm{n}$. sp.

10: Tricolpopollenites meinohamensis TAKAHASHI subsp. rotundus TAKAHASHI

11: Tricolpopollenites microreticulatus TAKAHASH

12: Tricolpopollenites minutiretiformis $\mathrm{n}$. sp.

13: Rhoipites ? minutireticulatus $\mathrm{n}$. sp.

14: Aquilapollenites-Gruppe.

Australien und Neuseeland wird über diese Form nicht berichtet. Sie scheint auf die Oberkreide bis zum Paläozän der nordhemisphärischen Gebiete, die den pazifischen Ozean umgeben, beschränkt zu sein. Die Formgattung Pentapollenites wurde von W. KRUTZSCH (1957) vorgeschlagen. Sie ist der Form von Aquilapollenites ähnlich.

Der Verfasser möchte diese Sporen- und Pollengruppe als unteres Hakobuchi-Pollenbild auffassen.

Zwei Feinsandsteine aus der oberen Hakobuchi-Schichtengruppe wurden zuerst im Eisenmörser zerkleinert und dann mit Fluorwasserstoffsäure behandelt.

Die Sporen und Pollen aus dieser Schichtengruppe bei der Talspere von Ooyubari verteilen sich in folgender Weise:

Probe G:

Sporites

Triletes

Gleicheniidites marginatus n. sp. .................... 1\% Concavisporites sp. b ........................ $1 \%$

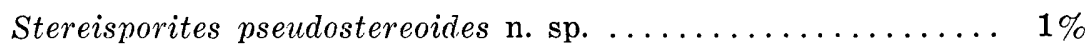

Corrugatisporites cf. solidus (R. Рот.) Tн. \& PF. ........ 1\%

Lycopodiumsporites yubariensis n. sp. ............. $2 \%$

Baculatisporites cf. validus n. sp. ................ 1\% 
Neoraistrickia sp. ........................ 1\%

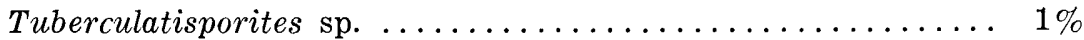

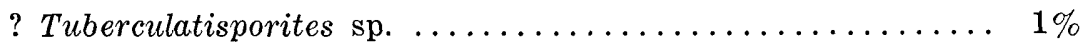

Zonales

Biretisporites ? minus n. sp. .................... $1 \%$ Monoletes

Laevigatosporites prominens n. sp. ............... $3 \%$

Laevigatosporites ? probatus n. sp. .............. 2\%

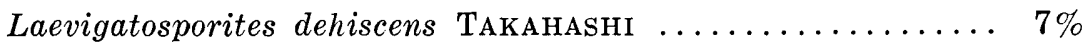

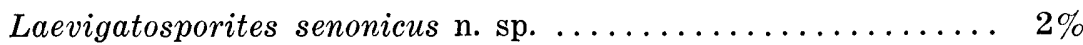

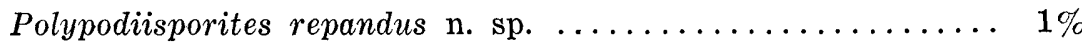

Polypodiisporites invisus n. sp. ................. 1\%

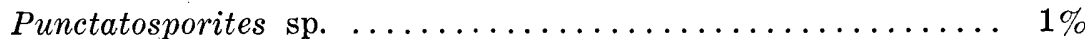
Pollenites

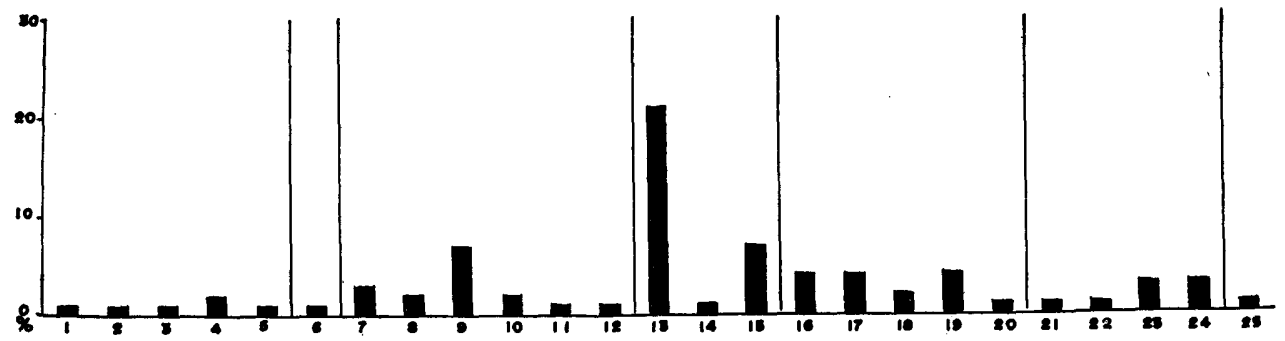

Abb. 7. Sporen- und Pollendiagramm der Probe G der oberen Hakobuchi-

Schichtengruppe.

1: Gleicheniidites marginatus n. sp.

2: Stereisporites pseudostereoides n. sp.

3: Corrugatisporites cf. solidus (R. Poт.) Thomson \& Pflug

4: Lycopodiumsporites yubariensis $\mathrm{n}$. sp.

5: Baculatisporites cf. validus n. sp.

6: Biretisporites ? minus n. sp.

7: Laevigatosporites prominens $\mathrm{n}$. sp.

8: Laevigatosporites ? probatus $\mathrm{n}$. sp.

9: Laevigatosporites dehiscens TAKAHASHI

10: Laevigatosporites senonicus $\mathrm{n}$. $\mathrm{sp}$.

11: Polypodiisporites repandus n. sp.

12: Polypodiisporites invisus n. sp.

13: Inaperturopollenites pseudodubius TAKAHASHI

14: Inaperturopollenites ? falsus n. sp.

15: Inaperturopollenites sp.

16: Betulaepollenites minutulus $\mathrm{n}$. sp.

17: Alnipollenites eminens (TAKAHASHI)

18: Polyporopollenites punctatus n. sp.

19: Ulmipollenites sp.

20: Graminidites microapertus n. sp.

21: Monocolpopollenites shiyuparoensis n. sp.

22: Tricolpopollenites cf. ditis TAKAHASHI

23: Rhoipites ? minutireticulatus n. sp.

24: Tricolporopollenites cf. minor TAKAHASHI

25: Aquilapollenites quadrinus n. sp. 
Aletes

Inaperturopollenites pseudodubius TAKAHASHI $\ldots \ldots \ldots \ldots 21 \%$

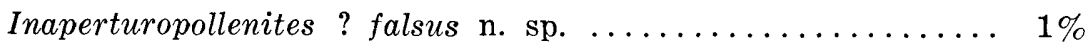

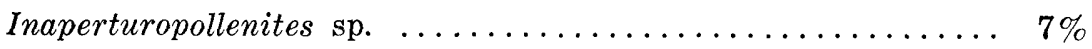

Poroses

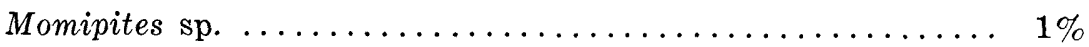

Betulaepollenites minutulus n. sp. .................. $4 \%$

Betulaepollenites sp. ....................... (+)

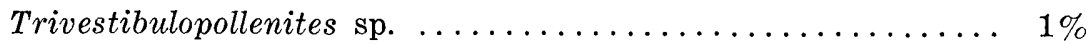

Alnipollenites eminens (TAKAHASHI) $\ldots \ldots \ldots \ldots \ldots \ldots \ldots \ldots$

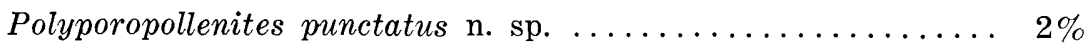

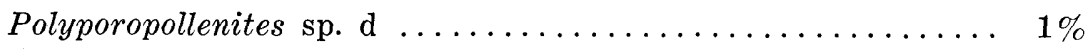

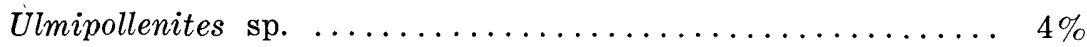

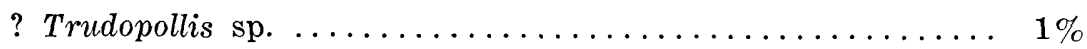

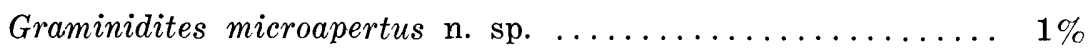

Plicates

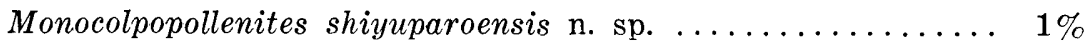

Tricolpopollenites $\mathrm{cf}$ ditis Takahashi $\ldots \ldots \ldots \ldots \ldots \ldots \ldots$

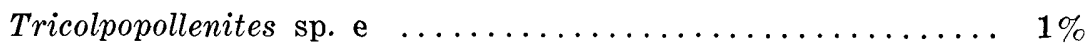

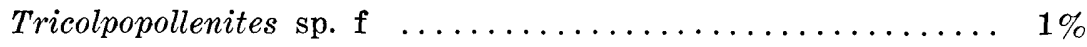

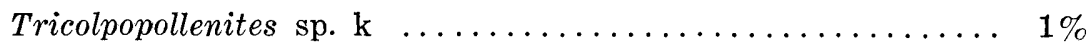

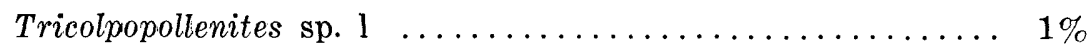

Rhoipites ? minutireticulatus n. sp. .................. $3 \%$

Tricolporopollenites cf. minor TAKAHASHI ............ $3 \%$

Incertae Sedis

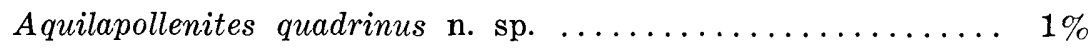

? Pistillipollenites sp. ....................... $1 \%$

Unbestimmbare Pollen $\ldots \ldots \ldots \ldots \ldots \ldots \ldots \ldots \ldots \ldots \ldots \ldots \ldots$

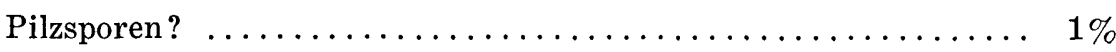

Probe $\mathrm{H}$ :

Sporites

Triletes

? Concavisporites sp. ........................ 1\%

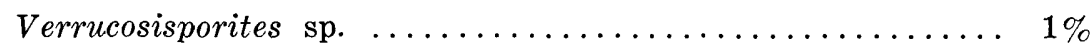

Rugulatisporites cf. salebrosus n. sp. ............. 1\%

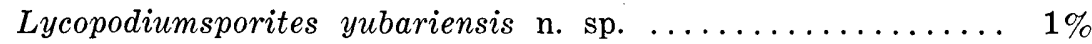

Apiculatisporis cf. inouei n. sp. ................... 1\%

Monoletes

Laevigatosporites dehiscens TAKAHASHI $\ldots \ldots \ldots \ldots \ldots \ldots 2 \%$

Laevigatosporites senonicus TAKAHASH $\ldots \ldots \ldots \ldots \ldots \ldots \ldots$

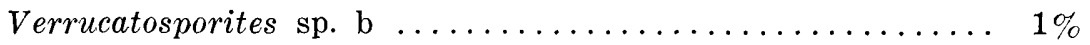

Pollenites

Aletes

Inaperturopollenites pseudorlubius TAKAHASHI $\ldots \ldots \ldots \ldots .9 \%$ 


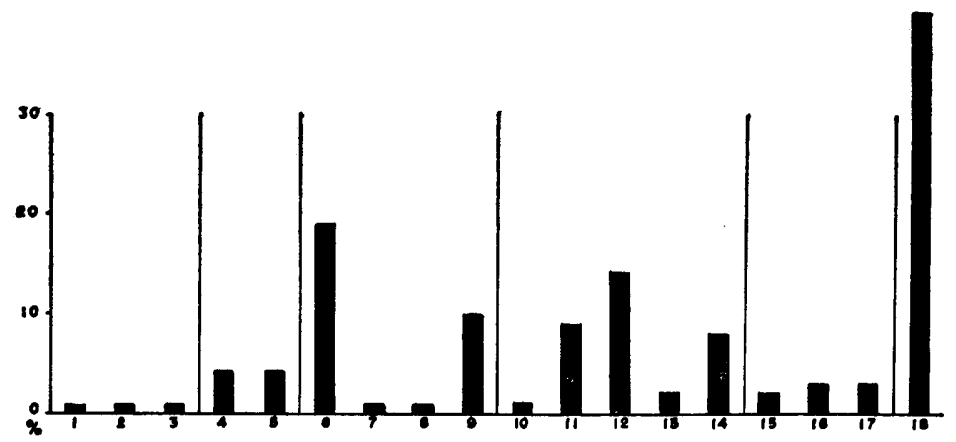

Abb. 8. Sporen- und Pollendiagramm der Probe $\mathrm{H}$ der oberen HakobuchiSchichtengruppe.

1: Rugulatisporites cf. salebrosus n. sp.

2: Lycopodiumsporites yubariensis $\mathrm{n}$. $\mathrm{sp}$.

3: Apiculatisporis cf. inouei n. sp.

4: Laevigatosporites dehiscens TAKAHASHI

5: Laevigatosporites senonicus $\mathrm{n}$. $\mathrm{sp}$.

6: Inaperturopollenites pseudodubius TAKAHASHI

7: Inaperturopollenites magnus (R. Рот.) THOMson \& PFLUG

8: Inaperturopollenites ? falsus $\mathrm{n}$. sp.

9: Inaperturopollenites parviundulatus $\mathrm{n}$. sp.

10: Betulaepollenites normalis $\mathrm{n}$. sp.

11: Betulaepollenites minutulus n. sp.

12: Alnipollenites eminens (TAKAHASHI)

13: Polyporopollenites punctatus n. sp.

14: Ulmipollenites sp.

15: Monocolpopollenites kyushuensis TAKAHASHI

16: Tricolpopollenites minutiretiformis $\mathrm{n}$. $\mathrm{sp}$.

17: Tricolporopollenites cf. minor TAKAHASHI

18: Pilzsporen?

Inaperturopollenites magnus (R. Pот.) THOMsON \& PFLUG .... $1 \%$ Inaperturopollenites ? falsus n. sp. ............... $1 \%$

Inaperturopollenites parviundulatus n. sp. ........... $5 \%$

Poroses

Betulaepollenites normalis $n$. sp. .............. $1 \%$

Betulaepollenites minutulus n. sp. ............... $4 \%$

? Triporopollenites sp. .................... $1 \%$

Alnipollenites eminens (TAKAHASHI) $\ldots \ldots \ldots \ldots \ldots \ldots \ldots$

Polyporopollenites punctatus n. sp. ............... $2 \%$

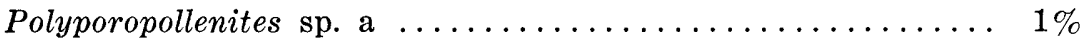

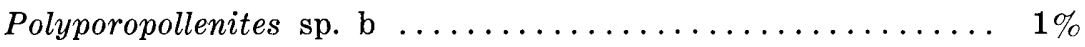

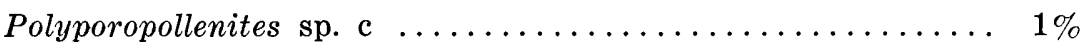

Polyporopollenites sp. e .................. $1 \%$

Polyporopollenites sp. f .................. 1\%

Ulmipollenites sp. ..................... $4 \%$

Plicates

Monocolpopollenites kyushuensis TAKAHASHI ......... $2 \%$ 
Tricolpopollenites minutiretiformis n. sp. ........... $3 \%$ Tricolporopollenites cf. minor TAKAHASHI ............ $3 \%$

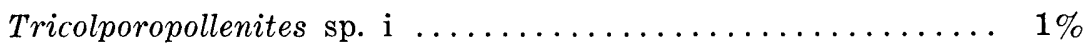

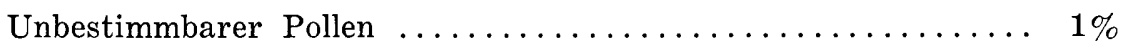

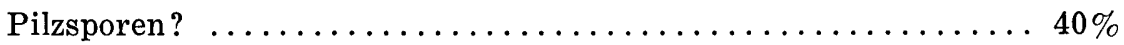

Im oberen Hakobuchi-Pollenspektrum treten besonders PolypodiaceenSporen, ungeflügelter Koniferen-Pollen (Inaperturopollenites pseudodubius), Betulaceae- und Ulmaceae-Pollen häufig auf. Es ist besonders bemerkenswert, daß die Artenzahl der Betulaceae- und Ulmaceae-Pollen höher ist als bei dem unteren Hakobuchi-Bild.

Man kann viele gemeinsame Spezies im unteren Hakobuchi-Pollenspektrum und im oberen Hakobuchi-Pollenspektrum finden; nämlich Gleicheniidites marginatus n. sp., Stereisporites pseudostereoides n. sp., Rugulatisporites salebrosus n. sp., Biretisporites ? minus n. sp., Apiculatisporis inouei n. sp., Baculatisporites validus n. sp., Laevigatosporites prominens n. sp., Laevigatosporites probatus n. sp., Laevigatosporites dehiscens TAKAHASHI, Laevigatosporites senonicus n. sp., Polypodiisporites repandus n. sp., Polypodiisporites invisus n. sp., Monocolpopollenites shiyuparoensis n. sp., Monocolpopollenites kyushuensis TAKAHASHI, Inaperturopollenites pseudodubius TAKAHASHI, Inaperturopollenites magnus (R. Pот.) Thomson \& Pflug, Alnipollenites eminens (TAKAhashi), Betulaepollenites normalis n. sp., Graminidites microapertus n. sp., Tricolpopollenites minutiretiformis n. sp., Rhoipites minutireticulatus n. sp., Tricolporopollenites minor TAKAHASHI.

Viele neue Spezies, die im unteren Hakobuchi-Pollenspektrum fehlen, kann man in diesem Spektrum bemerken.

Koniferen-Pollen mit Luftsack wurde hier gar nicht gefunden.

Aquilapollenites quadrinus TAKAHASHI ist die einzige Spezies von Aquilapollenites aus der oberen Hakobuchi-Schichtengruppe und sie scheint auf das obere Hakobuchi-Pollenspektrum beschränkt zu sein.

Der Verfasser möchte dieses oben beschriebene Pollenspektrum als oberes Hakobuchi-Pollenbild bezeichnen.

\section{B) Sporen und Pollen der Noborikawa-Schichten.}

Zwei Proben I und J der Steinkohlen der Noborikawa-Schichten wurden hier pollenanalytisch behandelt, aber der Verfasser hat gar keinen Pollen in der Probe I bemerkt. Deshalb kann er nur über den Befund der Probe J berichten.

Die Proben wurden zuerst im Eisenmörser zerkleinert und dann mit dem Schultzeschen Gemisch $\left(\mathrm{HNO}_{3}+\mathrm{KClO}_{3}\right)$, und nachfolgend mit Alkali behandelt. Um viele Minerale zu lösen, hat der Verfasser zuletzt Fluorwasserstoffsäure (HF) benutzt.

Die Sporen und Pollen der Noborikawa-Schichten sind in folgender Tabelle verzeichnet:

Probe J :

Sporites 


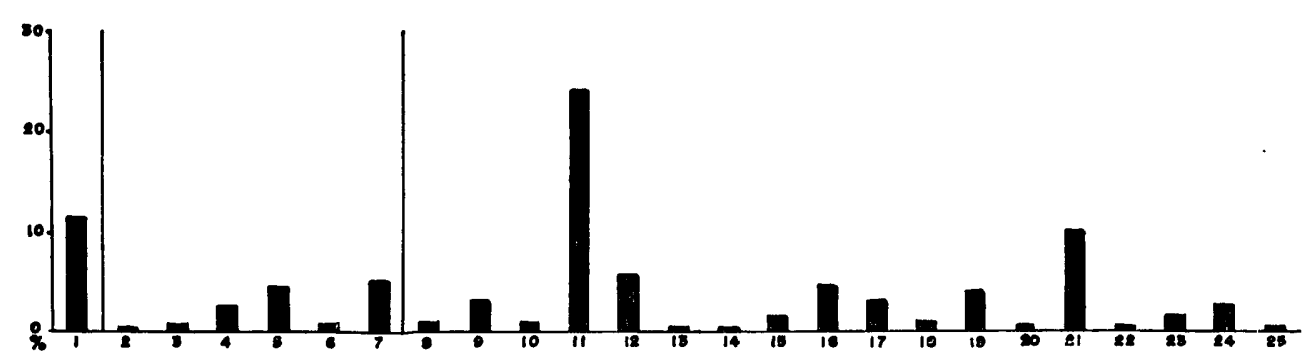

Abb. 9. Pollendiagramm der Noborikawa-Schichten bei der Talspere von Ooyubari.

1: Inaperturopollenites pseudodubius TAKAHASHI

2: Momipites constatus (TAKAHASHI)

3: Triporopollenites-Gruppe.

4: Subtriporopollenites kyushuensis TAKAHASHI

5: Alnipollenites eminens (TAKAHASHI)

6: Ulmipollenites undulosus WoLFF

7: Periporopollenites asiaticus TAKAHASHI

8: Monocolpopollenites universalis TAKAHASHI

9: Monocolpopollenites kyushuensis TAKAHASHI

10: Monocolpopollenites pflugii TAKAHASHI

11: Tricolpopollenites ditis TAKAHASHI

12: Tricolpopollenites vulgaris TAKAHASHI

13: Tricolpopollenites umiensis TAKAHASHI

14: Tricolpopollenites subasper TAKAHASHI

15: Tricolpopollenites inamoenus TAKAHASHI

16: Tricolpopollenites meinohamensis TAKAHASHI subsp. rotundus TAKAHASHI

17: Tricolpopollenites reticulatus TAKAHASHI

18: Tricolpopollenites microreticulatus TAKAHASHI

19: Tricolpopollenites rudis TAKAHASHI

20: Tricolpopollenites minutiretiformis $\mathrm{n}$. $\mathrm{sp}$.

21: Tricolpopollenites minutissmus n. sp.

22: Rhoipites ? hoshuyamaensis TAKAHASHI subsp. hoshuyamaensis TAKAHASHI $n$. emend.

23: Tricolporopollenites minor TAKAHASHI

24: Tricolporopollenites incertus TAKAHASHI

25: Ilexpollenites-Gruppe.

Triletes

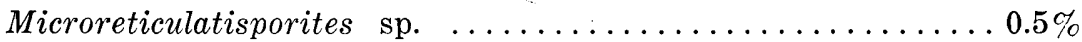

Pollenites

Aletes

Inaperturopollenites pseudodubius TAKAHASHI $\ldots \ldots \ldots \ldots \ldots 11.5 \%$

Inaperturopollenites shikokuensis TAKAHASHI ........... (+) Saccites

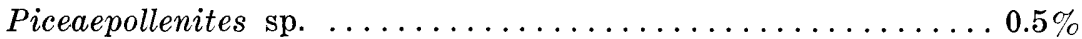

Poroces

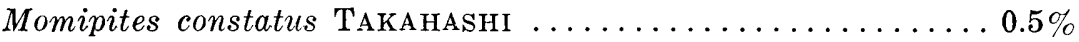

Triporopollenites cf. festatus TAKAHAsHi ........... $0.5 \%$ 
Triporopollenites shimensis TAKAHAsHr $\ldots \ldots \ldots \ldots \ldots \ldots \ldots$

Triporopollenites ? sp. .......................

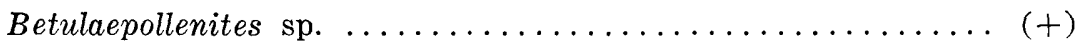

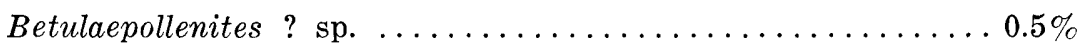

Subtriporopollenites kyushuensis TAKAHASHI ..........

Alnipollenites eminens (TAKAHASHI) n. comb. .......... $4.5 \%$

Ulmipollenites undulosus WOLFF ................ $1 \%$

Polyporopollenites grandis Takahashi ............ $0.5 \%$

Periporopollenites asiaticus Takahashi $\ldots \ldots \ldots \ldots \ldots \ldots \ldots$

Plicates

Monocolpopollenites universalis TAKAHASHI ........... 1\%

Monocolpopollenites kyushuensis TAKAHAsHI .......... $3 \%$

Monocolpopollenites pflugii TAKAHAshI ............ 1\%

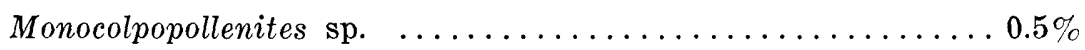

Tricolpopollenites ditis TAKAHASHI ............. 24\%

Tricolpopollenites vulgaris TAKAHASHI $\ldots \ldots \ldots \ldots \ldots \ldots . \ldots \ldots$

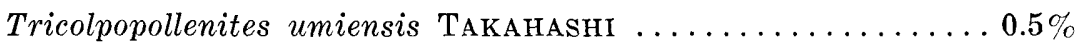

Tricolpopollenites subasper TAKAHASHI ............ $0.5 \%$

Tricolpopollenites inamoenus TAKAHASHI ............

Tricolpopollenites meinohamensis TAKAHASHI subsp.

rotundus TAKAHASHI ........... 4.5\%

Tricolpopollenites reticulatus TAKAHASHI ............ $3 \%$

Tricolpopollenites microreticulatus TAKAHASHI .......... 1\%

Tricolpopollenites rudis TAKAHASHI ............. $4 \%$

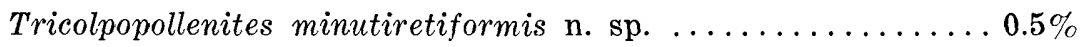

Tricolpopollenites minutissmus n. sp. ............. 10\%

Tricolpopollenites sp. ................... $0.5 \%$

Rhoipites ? hoshuyamaensis TAKAHAsHi n. comb. subsp.

hoshuyamaensis $\mathrm{n}$. emend. ........... $0.5 \%$

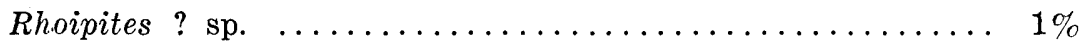

Tricolporopollenites minor TAKAHAsHI $\ldots \ldots \ldots \ldots \ldots \ldots \ldots$

Tricolporopollenites cf. cinglum (R. Pot.) Thomson \& PFLUG

subsp. fusus (R. Pot.) Thomson \& PFLUG .......... $2.5 \%$

Tricolporopollenites castaneoides TAKAHASHI ........... $1 \%$

Tricolporopollenites incertus TAKAHASHI ........... $2.5 \%$

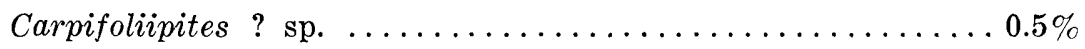

Ilexpollenites clavatus (TAKAHASHI) n. comb. .........5\%

Ilexpollenites excellens (TAKAHASHI) n. comb. ............. (t) Jugates

Ericipites sp. ...................... 1\%

Die meisten Pollen der Noborikawa-Schichten sind vom Verfasser (1957, 1961, 1962) aus dem Alttertiär und Miozän Westjapans beschrieben worden. $\mathrm{Zu}$ dieser Pollengruppe kann er auf folgende wichtige Tatsache hinweisen. Im Pollenspektrum der Noborikawa-Schichten sind zwei Pollengruppen vorherr- 
schend, nämlich der ungeflügelte Koniferen-Pollen (Inaperturopollenites pseudodubius TAKaHASHI) und die tricolpate Cupuliferen-Pollengruppe. Von der Cupuliferen-Pollengruppe ist Tricolpopollenites ditis TAKAHASHI am häufigsten. Untergeordnet folgt Tricolpopollenites vulgaris TAKAHASHI. Tricolpopollenites umiensis TAKAHASHI tritt hier sehr selten auf. Das Vorherrschen der zwei oben erwähnten Pollengruppen ist auch für das Alttertiär Westjapans charakteristisch. Das verhältnismäßig häufigere Auftreten der monocolpaten PalmaePollengruppe ist auch wichtig. Tricolpopollenites subasper TAKAHASHI hat der Verfasser bisher nur in den eozänen Schichten von Westjapan und den oberkretazeischen Schichten von Hokkaido bemerkt. Tricolpopollenites inamoenus TAKAHASHI hat er im Noogata-Pollenbild von Westjapan verhältnismäßig häufig und in den anderen alttertiären Pollenbildern selten gefunden. Tricolpopollenites rudis TaKaHashr wurde im Alttertiär von Westjapan und Nordjapan beschränkt gefunden. Tricolpopollenites minutiretiformis TAKAHASHI findet sich in den oberkretazeischen Schichten häufig. Hier ist diese Form sehr selten. Tricolpopollenites minutissmus $\mathrm{n}$. sp. ist eine sehr kleine Form. Sie scheint eine eigentümliche Form in den Noborikawa-Schichten zu sein, aber dies muß noch die Untersuchung der alttertiären Sporen und Pollen von Hokkaido stätigen. Rhoipites ? hoshuyamaensis TAKAHASHI subsp. hoshuyamaensis n. emend. (Tricolporopollenites hoshuyamaensis TAK. subsp. fossulatus TAK. 1961, S. 325, Taf. 25, Fig. 5-9) ist eine eozäne Form, die vom Verfasser im Ariake- und NoogataPollenbild von Westjapan festgestellt wurde. Es ist bemerkenswert, daß Periporopollenites asiaticus TAKAHASHI (Liquidambar) verhältnismäßig häufig auftritt. Inaperturopollenites shikokuensis TAKAHASHI (Salicaceae, Triuridaceae) ist der gleiche Pollen, der vom Verfasser (1962) aus den eozänen Schichten von Ishizuchi, Shikoku beschrieben wurde.

Sporen und geflügelte Koniferen-Pollen sind sehr selten.

Die Zusammensetzung der vorherrschend auftretenden Pollengruppen (Inaperturopollenites pseudodubius, Tricolpopollenites ditis und Tricolpopollenites vulgaris) und der altersmäßig wichtigen Pollen (Tricolpopollenites subasper, Tricolpopollenites inamoenus, Rhoipites ? hoshuyamaensis subsp. hoshuyamaensis, Inaperturopollenites shikokuensis u. a.) beweist dem Verfasser, daß das Pollenspektrum der Noborikawa-Schichten dem Noogata-Pollenbild von Westjapan sehr näher ähnlich ist. Aber der Vergleich mit den westjapanischen alttertiären Pollenbildern muß noch durch die eingehende Untersuchung der alttertiären Schichten im ganzen Ishikari-Kohlenfeld stätigt werden. Der Verfasser kann das vorliegende Pollenspektrum nur als vorläufiges „Noborikawa-Pollenbild“ bezeichnen.

\section{C) Oberkretazeische und alttertiäre Pollenbilder und ihr stratigraphisches Verhalten.}

Der Verfasser hat bisher über die Arten, Auftretensfrequenz und Eigentümlichkeiten der Sporen und Pollen der Hakobuchi-Schichtengruppe und der Noborikawa-Schichten berichtet. Infolgendessen hat er zwei Pollenbilder in der 
Hakobuchi-Schichtengruppe unterschieden und das „Noborikawa-Pollenbild“ für die Noborikawa-Schichten aufgestellt.

Im unteren $\mathrm{H}$ a kobuchi-Pollenbild kommen viele Sporen vor. Von ihnen sind Gleicheniaceae, Sphagnum-Sporen, Osmundaceae, Polypodiaceae u. a. vorherrschend. Von Pollen treten Weylandipollis retiformis, ungeflügelter Koniferen-Pollen, Alnus-Pollen, Tricolpopollenites minutiretiformis u. a. besonders hervor.

Concavisporites macellus n. sp., Stereisporites limbatus n. sp., Stereisporites grossus n. sp., Punctatisporites punctulatus n. sp., Apiculatisporis micracanthus n. sp., Baculatisporites papillosus n. sp., Laevigatisporites hokkaidoensis n. sp., Cicatricososporites ? ellipsoideus n. sp., Schizosporis sp. u. a. treten allein in diesem Pollenspektrum auf. Von Pollen kann man Weylandipollis retiformis $\mathrm{n}$. sp., Pityosporites aliformis (+microaliformis), Phyllocladidites brachypterus $\mathrm{n}$. sp. (+mirandus), Aquilapollenites triauritus n. sp., Aquilapollenites borealis n. sp., Aquilapollenites matsumotoi n. sp. u. a. nur in diesem Pollenbild finden.

Die Verteilung dieser stratigraphisch wichtigen Sporen und Pollen kann man aus der Abbildung 10 ersehen.

Der Verfasser konnte das obere Hakobuchi-Pollenbild von dem unteren Hakobuchi-Pollenbild sehr leicht unterscheiden.

Die vorherrschenden Sporen und Pollen in diesem Pollenspektrum sind Polypodiaceae-Sporen, ungeflügelter Koniferen-Pollen, Betulaceae- und UlmaceaePollen.

Die Hauptsporen und -pollen, die nur in diesem Spektrum auftreten, sind folgende Spezies: Corrugatisporites cf. solidus (R. PotoniÉ) THOMson \& PFLug, Lycopodiumsporites yubariensis n. sp., Inaperturopollenites ? falsus n. sp., Inaperturopollenites parviundulatus n. sp., Betulaepollenites minutulus n. sp., Polyporopollenites punctatus n. sp., Aquilapollenites quadrinus n. sp. u. a. (vgl. Abb. 10). Nach diesen Spezies kann man hauptsächlich das obere HakobuchiPollenbild von anderen Pollenbildern unterscheiden.

Es gibt viele gemeinsame Sporen- und Pollenarten in der unteren und oberen Hakobuchi-Schichtengruppe, aber von ihnen sind besonders Gleicheniidites marginatus n. sp., Stereisporites pseudostereoides n. sp., Apiculatisporis inouei n. sp., Baculatisporites validus n. sp., Polypodiisporites repandus n. sp., Monocolpopollenites shiyuparoensis n. sp., Betulaepollenites normalis n. sp. und Tricolpopollenites minutiretiformis $\mathrm{n}$. $\mathrm{sp}$. in dem unteren Hakobuchi-Pollenbild häufiger, in dem oberen Hakobuchi-Bild seltener.

Die merkwürdigen Neulinge der Sporen- und Pollengattungen Cicatricososporites, Schizosporis, Aquilapollenites, Phyllocladidites u. a. sind ihrer weltweiten Verbreitung nach und pollenfeinstratigraphisch sehr wichtig. Im nächsten Abschnitt schildert der Verfasser ihr stratigraphisches Auftreten und ihre geographische Verbreitung in einigen Gegenden der Welt.

Das Pollenspektrum aus den Noborikawa-Schichten vorläufig kann als „, Noborikawa-Polle n bild " bezeichnet werden.

Der Verfasser hat im Jahre 1961 die westjapanischen alttertiären und 
miozänen Pollenbilder festgestellt. Dabei hat er betont, daß es im Alttertiär von Westjapan zwei vorherrschende Pollengruppen gibt; nämlich ungeflügelte Koniferen-Pollen und die tricolpate Cupuliferen-Pollengruppe. Im NoborikawaPollenspektrum bemerkt er dieselben Eigentümlichkeiten. Das ist sehr wichtig. Ungeflügelter Koniferen-Pollen tritt mit $11.5 \%$ auf, während die tricolpate Cupuliferen-Pollengruppe vorherrscht. Von der letzteren ist Tricolpopollenites ditis TAKAHASHI am häufigsten. Untergeordnet kommt Tricolpopollenites vulgaris TAKAHASHI vor. Tricolpopollenites umiensis TAKAHASHI tritt nur wenig auf. Die meisten Pollen sind schon vom Verfasser aus dem Paläogen und Miozän von Westjapan beschrieben wurde. Außer diesen Pollengruppen sind vor allem Tricolpopollenites subasper TAKAHASHI, Tricolpopollenites inamoenus TAKAHASHI, Rhoipites ? hoshuyamaensis TAKAHASHI subsp. hoshuyamaensis TAKAHASHI und Inaperturopollenites shikokuensis TAKAHASHI bei der Vergleichung des Pollenbildes von Bedeutung. Tricolpopollenites subasper TAKAHASHI tritt im Ariake- und Noogata-Pollenbild von Westjapan beschränkt auf, häufiger besonders im Noogata-Pollenbild. Rhoipites ? hoshuyamaensis TAKAHASHI subsp. hoshuyamaensis TAKAHASHI kann man auch im Ariake- und NoogataPollenbild beschränkt finden. Er ist im Ariake-Pollenbild häufig. Tricolpopollenites inamoenus TAKAHASHI ist eine westjapanische alttertiäre Form, aber er tritt besonders im Noogata-Pollenbild häufiger auf. Inaperturopollenites shikokuensis TAKAHASHI hat man bisher nur in den obereozänen MyojinSchichten von Ishizuchi (Shikoku) gefunden.

Die monocolpate Palmae-Pollengruppe kann man verhältnismäßig öfter finden. Das ist wichtig. Monocolpopollenites universalis TAKAHASHI ist eine alttertiäre Form.

Tricolpopollenites rudis TAKAHASHI ist eine westjapanische paläogene Form. Er tritt verhältnismäßig häufig auf. Tricolpopollenites minutiretiformis TAKAHASHI n. sp. ist in der Oberkreide häufig, aber hier sehr selten. Die kleine Form, Tricolpopollenites minutissmus, ist ein Neuling. Sie kann man im Präparat oft finden.

. Die drei- bis vieleckige Pollengruppe umfaßt eine große Zahl von Arten. Davon tritt Alnipollenites eminens (TAKAHASHI) oft auf, andere Spezies nur mit einem geringen Prozentsatz.

Periporopollenites asiaticus TAKAHASHI (Liquidambar) kann man oft bemerken. Diese Form ist im Alttertiär und Miozän von Westjapan durchläufig vorhanden, aber sie ist im Eozän häufiger.

Sporen sind sehr selten. Es wurde hier nur ein Sporenkorn von Microreticulatisporites gefunden.

Nach den oben erwähnten Eigentümlichkeiten des Pollenauftretens möchte der Verfasser behaupten, daß das „Noborikawa-Pollenbild“ dem Noogata-Pollenbild sehr nahe steht. Dies muß aber noch durch die zusammenfassende Untersuchung der alttertiären Sporomorphae im Ishikari-Kohlenfeld bestätigt werden.

Die beide Pollenbilder der nordjapanischen Oberkreide können hier mit einigen ausländischen oberkretazeischen Pollenspektren verglichen werden. 


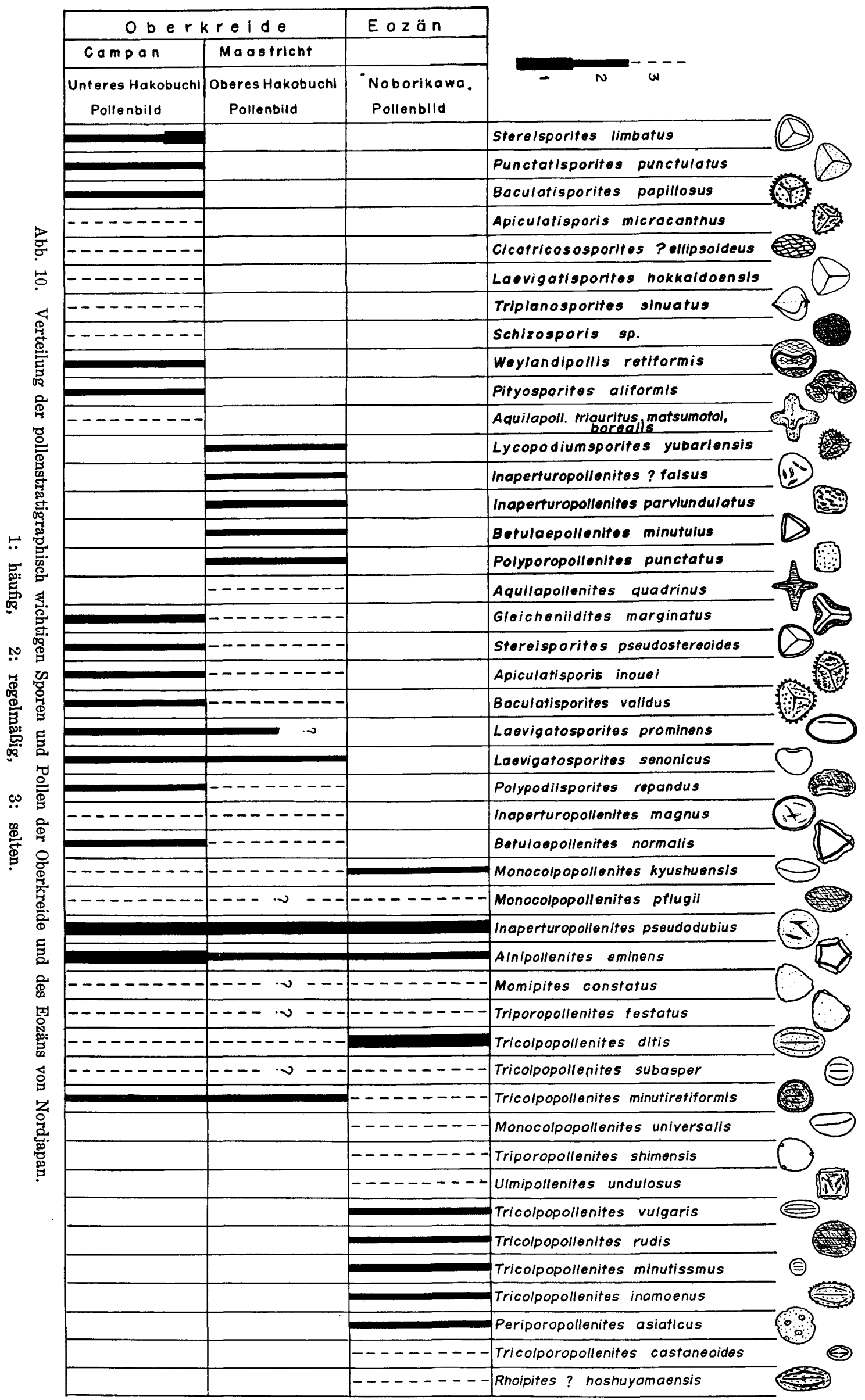


G. E. Rouse (1957) hat viele Sporen und Pollen aus den westkanadischen campanischen Comox- und Oldman-Schichten abgebildet und beschrieben. Der Vergleich der betreffenden und westkanadischen Sporomorphae ist sehr bedeutsam, weil die Comox- (oder Oldman-) und Hakobuchi-Schichten in ähnlicher Breite liegen.

Die Sporomorphae aus den campanischen Comox-Schichten in der Vancouver Insel sind folgende: Stereisporites (al. Sphagnum) antiquasporites (WILsoN \& WEBSTER), Stereisporites (al. Stenozonotriletes) simplex (NAUmova), Corrugatisporites (al. Sporites) solidus (R. Pот.) Thомson \& Pflug, Cibotiumsporites concavus Rouse, Laevigatosporites ovatus WILsON \& WEBSTER, Acanthotriletes typicus Naumova, Deltoidospora diaphana Wilson \& WeBster, Deltoidospora rhytisma Rouse, Verrucosisporites verrucosus IBRAHIM, Osmundacidites (al. Osmundasporites) sp., Laevigatisporites (al. Sporites) neddeni (R. Рот.), Perotrilites granulatus COUPER, Microreticulatisporites irregularis RoUsE, Pityosporites (al. Pinus haploxylon)-Typus, Beaupreadites elegansiformis Cookson, Tetracolporopollenites (al. Pollenites) manifestus (R. Pот.) THомson \& Pflug, Triporopollenites (al. Pollenites) megagranifer (R. Рот.), Triporopollenites (al. Engelhardtia) spackmanius (TRAVARSE), Tricolpopollenites (al. Pollenites) laesus (R. Рот.), Sparganiaceaepollenites (al. Sparganium) globipites (WILson \& WEBSTER), Tricolpopollenites minor ROUSE, Tricolpopollenites divergens RousE, Tricolpopollenites sp. u. a.

Von diesen Sporomorphae treten Corrugatisporites solidus (R. Рот.) Тном3ON \& Pflug und Laevigatosporites ovatus WILsON \& WeBsteR in der HakobuchiSchichtengruppe gemeinsam auf. Die Sporomorphae der Gattung Stereisporites (al. Sphagnum) sind den Formen derselben Gattung im unteren HakobuchiPollenbild sehr ähnlich. Die Spore, die von G. E. RousE als Verrucosisporites verrucosus IBRAHIM bezeichnet wurde, ist unserer Form Rúgulatisporites salebrosus TAKAHASHI ähnlich. Der morphologische Charakter von Acanthotriletes typicus Naumova steht dem von Tuberculatisporites sp. (Taf. 40, Fig. 9a, b) nahe. Microreticulatisporites nobilis (KNOX) PotoniÉ \& KREMP ist unserer Form Microreticulatisporites ? sp. (Taf. 26, Fig. 10) ähnlich. Die PerotrilitesForm wurde in der Hakobuchi-Schichtengruppe nicht gefunden.

Die Sporen und Pollen aus den campanischen Oldman-Schichten von Südalberta sind folgende Spezies: Stereisporites (al. Sphagnum) antiquasporites (WILSON \& WEBSTER), Stereisporites (al. Stenozonotriletes) simplex (NAUMOVA), Lycopodiumsporites (al. Lycopodium) papillaesporites (RoUsE), Lycopodiumsporites ? (al. Lycopodium) vermiculaesporites (RoUSE), Osmundacidites (al. Osmundasporites) primarius (WoLFF), Osmundacidites (al. Osmundasporites) elongatus (Rouse), Cicatricosisporites dorogensis R. Potonié \& Gelletich, Gleicheniidites (al. Gleichenia) concavisporites (RoUSE), Hymenophyllumsporites deltoida Rouse, Laevigatosporites albertensis Rouse, Laevigatosporites discordatus Thomson \& Pflug, Polypodiisporites (al. Polypodiaceaesporites) favus (R. Pot.), Verrucatosporites (al. Polypodiaceaesporites) alienus (R. PoT.), Deltoidospora diaphana WILSON \& WEBSTER, Microreticulatisporites microtuberosus 
(Loose) Potonié \& Kremp, Cf. Cyclogranisporites leopoldi (Kremp) PotoniÉ \& KRemp, Cf. Dictyotriletes densoreticulatus PotoniÉ \& KREMP, Cycadopites follicularis WiLson \& WeBster, Monocolpopollenites ? (al. Ginkgo) labiapollenites (Rouse), Inaperturopollenites (al. Taxodium) hiatipites (WodeHouse), Podocarpidites biformis Rouse, Pityosporites (al. Pinus) tuberculipites (WoDEHouse), Piceaepollenites (al. Picea) grandivescipites (WoDEHOUse), Heliotropium lobopollenites ROUSE, Sapindus circularopollenites ROUSE, Liliacidites variegatus CoUPER, Triporopollenites (al. Corylus) punctatipollenites (RoUsE), Triporopollenites (al. Engelhardtia) triletipollenites (RousE), Elytranthe. stratus CoUPER, Tricolporopollenites (al. Castanea) minutapollenites (RousE), Tricolpopollenites liblarensis (Thomson) Thomson \& PFLUG, Sporonites montanensis Miner, Aquilapollenites quadrilobus Rouse, Aquilapollenites trialatus Rouse, Trifossapollenites ellipticus Rouse u. a.

Von diesen Sporomorphae kann man Cicatricosisporites dorogensis R. Potoní́ \& Gelletich in der unteren Hakobuchi-Schichtengruppe finden. Die oben zitierten Speziesformen von Stereisporites, Lycopodiumsporites, Gleicheniidites, Laevigatisporites, Polypodiisporites, Verrucatosporites, Microreticulatisporites, Inaperturopollenites, Podocarpidites, Piceaepollenites und Aquilapollenites stehen denen aus der Hakobuchi-Schichtengruppe morphologisch nahe. Besonders ist des Auftreten von Aquilapollenites sehr wichtig (vgl. nächsten Abschnitt).

Von den Sporomorphae der Oberkreide von West-USA kennt der Verfasser fast nur die außergewöhnliche Pollenform Aquilapollenites von FunkHousER (1961) und StANLEY (1961).

J. J. Groot \& J. S. Penny (1960) und J. J. Groot, J. S. Penny \& C. R. GRоoт (1961) haben viele Sporen und Pollen der präcampanischen Kreide von Ost-USA (Maryland, Delaware, New Jersey, North Carolina, South Carolina und Georgia) beschrieben. J. J. Groot und C. R. Groot (1962) haben über die pflanzlichen Mikrofossilien aus den paläozänen Brightseat-Schichten von Maryland berichtet.

Leider hat der Verfasser selbst keine Kenntnis von den Sporen und Pollen des Campans und Maastrichts von Ost-USA. Er ist daher auf die Arbeiten von Groot und PENNy angewiesen. Von diesen präcampanischen und paläozänen Sporomorphae kann man einige Normapolles-Pollen, deren beschränktes Auftreten in der Oberkreide und dem tiefen Alttertiär von Europa von H. D. PFLuG (1953), W. KRUTzsch (1957) u. a. betont wurde, finden. Dagegen kann man sie in den Pollenspektren der Oberkreide von Nordjapan und Westkanada nicht feststellen. Das ist sehr wichtig. Die Normapolles-Pollen von Ost-USA sind folgende Spezies: Kreide-Turonipollis americanus J. Groot, PennY \& C. Groot, Plicapollis magnus J. Groot, Penny \& C. Groot, Plicapollis serta Pflug, Plicapollis vulgaris J. GROOT, PENNY \& C. GROOT, Sporopollis laqueaeformis Weyland \& Greifeld, Quedlinburgipollis minimus J. Groot, Penny \& C. Groot, Trudopollis convector PfLUG, Vacuopollis spp.; Paläozän-Latipollis conspicuus J. \& C. Groot, Extratriporopollenites audax PFLUG, Extratriporopollenites sp., 


\section{Plicapollis silicatus PfLUG.}

In den europäischen oberkretazeischen und tief-alttertiären Formationen kann man ausnahmslos die Normapolles-Pollengruppe finden. Von diesem Standpunkt aus kann man die Provinz der pflanzlichen Mikrofossilien der Oberkreide und des Paläozäns von Ost-USA zu der von Europa stellen. Dagegen möchte man West-USA, Westkanada, Japan u. a. in eine besondere Provinz einordnen. In der Oberkreide und dem tiefen Paläozän von Australien und Neuseeland kann man ebenfalls keinen Normapolles-Pollen bemerken.

\section{D) Geographische Verbreitung der wichtigen nachweisbaren Sporen und Pollen.}

In diesem Abschnitt erläutert der Verfasser die geographische Verbreitung der Sporen- und Pollenarten Cicatricosisporites dorogensis R. РотоNIÉ \& GeLLETICH, Corrugatisporites solidus (R. Рот.) Thomson \& PfLUG, Laevigatosporites ovatus Wilson \& Werster, Schizosporis, Phyllocladidites, Aquilapollenites und Pentapollenites.

Die Abbildung 11 illustriert die geographische Verbreitung jeder Art.

\section{1) Cicatricosisporites dorogensis R. Potoní \& Gelletich}

Diese trilete Form wurde zuerst von R. PotoniÉ und J. Gelletich aus den oberpaläozänen bis eozänen Schichten von Dorog, Ungarn beschrieben. Die Abbildung zeigt die sehr weite Verbreitung im Tertiär und Mesozoikum.

Europa:

R. Potonié und J. Gelletich, 1933, S. 522, Taf. 1, Fig. 1. Dorog, Ungarn; Oberes Paläozän bis Eozän.

R. Ротоní́, 1934, Taf. 1, Fig. 21. Geiseltal; Eozän.

F. Thiergart, 1940, S. 24, Taf. 6, Fig. 1,; Taf. 7, Fig. 25; Taf. 8, Fig. 1, 2, 5, 6, 9 . Ellenhausen; Wahrscheinlich Mittel-Unteroligozän.

Bohrung Ziegenhain bei Kassel; Unteroligozän. Westerwald; Unteroligozän.

P. W. Thomson und H. D. Pflug, 1953, S. 48-49, Taf. 1, Fig. 1-12. Antweiler Graben, Helmstedt, Borken, Kettig; Paläozän bis Oligozän.

A. Delcourt und G. Sprumont, 1955, S. 21, Abb. 3. Hainaut, Belgien; Wealden.

W. KRUtzSCH, 1957, S. 514, Taf. 1, Fig. 22-26. Pirna, Dorog, Zeitz, Deuben; Alttertiär.

R. A. Couper, 1958, S. 138, Taf. 17, Fig. 10-12. England; Purbeck-Schichten, Wealden.

West-Kanada:

G. E. Rouse, 1957, S. 362 , Taf. 2, Fig. $38,39$. Süd-Alberta; oldman-Schichten, Campan.

S. A. J. Рососк, 1962, S. 39, Taf. 2, Fig. 35-36; Taf. 3, Fig. 37-41. Süd-Alberta und Saskatchewan; Mannville und Colorado-Schichten, Neocom bis Senon.

Süd-USA :

W. KRUtZSCH, 1960, Taf. 5, Fig. 116. Austin, Texas; Wilcox-Stufe.

Ost-USA:

J. J. Groot und J. S. Penny, 1960, S. 230, Taf. 1, Fig. 2. Hawkins Point, Maryland; vielleicht Cenoman.

Tidewater Oil Co. well no. 12 


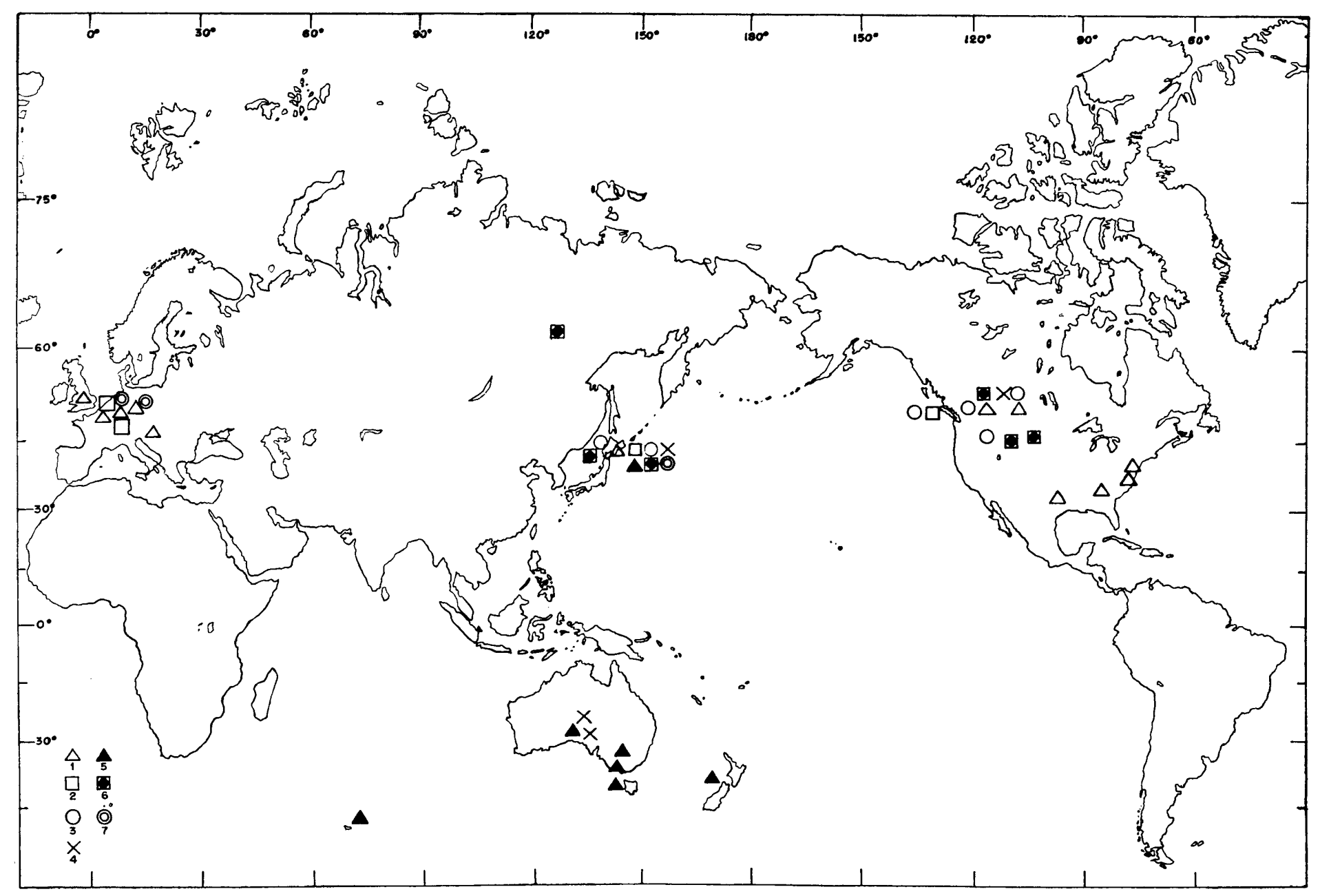

Abb. 11. Verbreitungskarte der wichtigen und merklichen Sporen und Pollen.
1: Cicatricosisporites dorogensis R. POTONIÉ \& GELLETICH
2: Corrugatisporites solidus (R. PotonIÉ) THOMSON \& PFLUG
3: Laevigatosporites ovatus WILSON \& WEBSTER 4: Schizosporis
5: Phyllocladidites 6: Aquilapollenites 7: Pentapollenites 
J. J. Groot, J. S. Penny und C. R. Groot, 1961, S. 128, Taf. 24, Fig. 8.

Alabama; Tuscaloosa-Schichten, vielleicht Cenoman.

SE von Milltown, New Jersey; Sayrenille, New Jersey; vielleicht Cenoman.

Georgia; Tuscaloosa-Schichten, etwas älter als Turon-Untersenon.

Ost-Riverton, New Jersey; Raritan-Magothy-Schichten.

Cliffwood Beach, New Jersey; Magothy-Schichten, Turon-Untersenon.

Chesapeake und Delaware Kanal, Maryland; Magothy-Schichten, Turon-Untersenon.

Für Australien gibt es keine Angabe über Cicatricosisporites dorogensis, wohl aber über eine sehr ähnliche Form Cicatricosisporites australiensis.

I. C. Cookson, 1954, S. 122, Fig. 2d.

Birregurra, Victoria; Kreide.

I. C. Cookson, 1953, S. 470, Taf. 2, Fig. 31-34.

Japan:

Comaum, S. Australien; Prätertiär.

K. Takahashi (in dieser Arbeit), S. 190, Taf. 23, Fig. 1-3.

Ooyubari, Yubari-Kohlenfeld, Hokkaido; Untere Hakobuchi-Schichtengruppe, Campan.

2) Corrugatisporites solidus (R. Potoní) Thomson \& Pflug

Die solidus-Form wurde von R. PотоNIÉ (1934) erstmalig aus den eozänen Braunkohlen des Geiseltals beschrieben. Ihre geographische Verbreitung ist verhältnismäßig weit.

Europa:

R. Potonié, 1934, S. 42, Tab. 1, Fig. 35.

Geiseltal; Eozän.

P. W. Thomson und H. D. Pflug, 1953, S. 55.

subsp. multivallatus Pflug, S. 56, Taf. 2, Fig. 37-40.

Köln, Ville, Wallensen; Pliozän bis Miozän.

subsp. paucivallatus PfLUG, S. 56, Taf. 2, Fig. 41-43.

Eschweiler, Wehmingen; Miozän, Paläozän.

W. Krutzsch, 1957, Taf. 2, Fig. 10-20.

Gröbitz, Spremberg, Sabroth; (Paläozän bis Miozän).

H. Weyland und K. Takahashi, 1961, subsp. multivallatus Pflug, Taf. 43, Fig. 1.

Heerlen, Holländisch Limburg; (?) unteres Miozän.

West-Kanada:

G. E. Rouse; 1957, S. 354, Taf. 1, Fig. 27, 28.

Vancouver Insel; Comox-Schichten, Campan.

Japan:

K. Takahashi (in dieser Arbeit), S. 203, Taf. 40, Fig. 5 .

Ooyubari, Yubari-Kohlenfeld, Hokkaido; Obere Hakobuchi-Schichtengruppe, Maastricht.

\section{3) Laevigatosporites ovatus WiLson \& WeBster}

Diese Form wurde zuerst von L. R. WiLson und R. M. Webster (1946) aus den paläozänen Fort Union-Schichten von Montana (USA) beschrieben. Sie wurde bisher nur aus den pazifischen Randgebieten mitgeteilt.

West-USA :

L. R. Wilson und R. M. Webster, 1946, S. 273, Fig. 5.

Kolarich Grube bei Red Lodge, Montana; Fort Union-Schichten, Paläozän.

West-Kanada:

G. E. Rouse, 1957, S. 355, 364, Taf. 1, Fig. 3; Taf. 2, Fig. 19-20.

Vancouver Insel; Comox-Schichten, Campan. 
Süd-Alberta; Oldman-Schichten, Campan.

G. E. Rouse, 1962, S. 198, Taf. 2, Fig. 1-2.

Third Beach, Terminal Dock und Brothers Creek; Burrard-Schichten, Mittel- bis Obereozän (und Oberkreide).

S. A. J. Рососк, 1962, S. 58, Taf. 8, Fig. 130-132.

Süd-Alberta, Saskatchewan; Oberjura und Kreide. Japan:

S. SATo, 1961, Taf. 2, Fig. 20-21.

Enbetsu, Nordhokkaido; Osoushinai-Schichten, Oberkreide.

K. Takahashi (in dieser Arbeit), S. 216, Taf. 29, Fig. 20.

Ooyubari, Yubari-Kohlenfeld, Hokkaido; Untere Hakobuchi-Schichtengruppe, Campan.

\section{4) Schizosporis}

Die Gattung Schizosporis wurde zuerst von I. C. Cookson und M. E. DETTMANN (1959) vorgeschlagen. Diese Form wurde bisher nur aus den pazifischen Randgebieten beschrieben.

Australien:

Schizosporis reticulatus Cookson \& DETTMANN.

I. C. Cookson und M. E. Dettmann, 1959, S. 216, Taf. 1, Fig. 1-4.

S. Australien; Winton-Schichten, Ober-Kreide (Cenoman?)

Schizosporis parvus CoOKSON \& DETTMANN

I. C. Cookson und M. E. Detrmann, 1959, S. 216, Taf. 1, Fig. 15-20.

S. Australien; Winton-Schichten, Oberkreide (Cenoman?)

Schizosporis rugulatus CoOKSON \& DETTMANN

I. C. Cookson und M. E. Dettmann, 1959, S. 216, Taf. 1, Fig. 5-9.

S. Australien; Unterkreide (Alb).

Schizosporis spriggi CooKson \& DetTManN

S. Australien; Tambo-Schichten, Unterkreide (Alb).

West-Kanada:

Schizosporis cooksoni Pocock

S. A. J. Рососк, 1962, S. 76, Taf. 13, Fig. 197-198.

Süd-Alberta, Saskatchewan; Oberjura und Basiskreide (Neokom).

Schizosporis grandis Pocock

S. A. J. Рocock, 1962, S. 76, Taf. 13, Fig. 199.

Süd-Alberta, Saskatchewan; Unter-Mannville-Schichten, Barrem.

Schizosporis parvus CooKson \& DETTMANN

S. A. J. Рососк, 1962, S. 76, Taf. 13, Fig. 200-201.

Süd-Alberta, Saskatchewan; Ober-Neokom.

Schizosporis reticulatus Cookson \& DeTTMANN

S. A. J. Рососк, 1962, S. 76, Taf. 13, Fig. 202.

Süd-Alberta, Saskatchewan; Ober-Neokom.

Schizosporis rugulatus CoOKSN \& DetTMANN

S. A. J. Рососк, 1962, S. 76-77, Taf. 13, Fig. 203-204.

Süd-Alberta, Saskatchewan; Ober-Neokom.

Schizosporis spriggii Cookson \& DetTMANN

S. A. J. Pocock, 1962, S. 77, Taf. 13, Fig. 205.

Süd-Alberta, Saskatchewan; Unter-Mannville bis Unter-

Colorado-Schichten, Neokom bis Alb.

Japan :

Schizosporis sp.

K. TAKahashi (in dieser Arbeit), S. 221, Taf. 31, Fig. 11a, b.

Ooyubari, Yubari-Kohlenfeld, Hokkaido; Untere Hakobuchi-Schichtengruppe, Campan. 


\section{5) Phyllocladidites}

Kerguelen Insel :

Phyllocladidites (al. Disaccites) mawsoni (Cookson)

I. C. Cookson, 1947, S. 133, Taf. 14, Fig. 22-28.

Kerguelen Insel; Tertiär.

Phyllocladidites (al. Disaccites) Rüei (Cookson)

I. C. Cookson, 1947, S. 133, Taf. 13, Fig. 31-37.

Kerguelen Insel; Tertiär.

Australien :

Phyllocladidites (al. Dacrydiumites) mawsonii (CooKson)

I. C. Cookson, 1953, S. 66, Taf. 1, Fig. 9-26.

NSW, Vic., SA, Tas.; Tertiär.

I. C. Cookson, 1953, S. 465, Taf. 1, Fig. 10.

S. Australien, Comaum; Alttertiär.

Phyllocladidites (al. Dacrydiumites) rüei (Cookson)

I. C. Cookson, 1953, S. 66, Taf. 1, Fig. 27.

NSW, Vic., SA, Tas.; Tertiär.

Neuseeland :

Phyllocladidites mawsoni (Cookson)

R. A. Couper, 1953, S. 38, Taf. 9, Fig. 135.

Neuseeland; Unterkreide bis Unteroligozän.

Japan :

Phyllocladidites brachypterus n. sp.

K. TaKahashi (in dieser Arbeit), S. 227-228, Taf. 35, Fig. 9, 10, 14.

Ooyubari, Yubari-Kohlenfeld, Hokkaido; Untere Hakobuchi-Schichtengruppe, Campan.

Phyllocladidites mirandus $\mathrm{n}$. sp.

K. TAKAhashi (in dieser Arbeit), S. 228, Taf. 35, Fig. 11-13.

Ooyubari, Yubari-Kohlenfeld, Hokkaido; Untere Hakobuchi-Schichtengruppe, Campan.

\section{6) Aquilapollenites}

West-Kanada:

Aquilapollenites quadrilobus Rouse

G. E. Rouse, 1957, S. 371, Taf. 2, Fig. 8-9.

Alberta; Brazeau-Schichten, Oberkreide.

Aquilapollenites trialatus Rouse

G. E. Rouse, 1957, S. 371, Taf. 2, Fig. 14-15.

Alberta; Oberkreide.

West-USA :

Aquilapollenites spinulosus FUNKHOUSER

J. W. FunkhousR, 1961, S. 194, Taf. 1, Fig. 4-6.

Rawlins, Wyoming; Fort Union-Schichten, Paläozän.

Aquilapollenites attenuatus FUNKHOUSER

J. W. Funkhouser, 1961, S. 194, 196, Taf. 2, Fig. 1a-c.

Rawlins, Wyoming; Lance-Schichten, Maastricht.

Aquilapollenites novacolpites FUNHOUSER

J. W. Funkhouser, 1961, S. 196, Taf. 2, Fig. 2-3.

Rawlins, Wyoming; Mesa Verde-Schichten, Campan.

Aquilapollenites bertillonites FUNKHOUSER

J. W. Funkhouser, 1961, S. 196, Taf. 2, Fig. 5a-c.

Rawlins, Wyoming; Lance-Schichten, Maastricht.

Aquilapollenites striatus FunKHOUSER

J. W. Funkhouser, 1961, S. 196, 198, Taf. 2, Fig. 4.

Rawlins, Wyoming; Lance-Schichten, Maastricht. 
Aquilapollenites polaris FUNKHOUSER

J. W. Funkhouser, 1961, S. 198, Taf. 1, Fig. 1-2.

Rawlins, Wyoming; Lanee-Schichten, Maastricht.

Aquilapollenites amplus STANLEY

E. A. Stanley, 1961, S. 342, 344, Taf. 1, Fig. 1-6; Taf. 2, Fig. 1-4; Taf. 3, Fig.

$1-5$.

Crow Butte, S-Dakota; Hell Creek-Schichten, Maastricht. Aquilapollenites delicatus STANLEY

E. A. Stanley, 1961, S. 346, Taf. 4, Fig. 1-12.

Crow Butte, S-Dakota; Hell Creek-Schichten, Maastricht. Aquilapollenites murus STANLEY

E. A. Stanley, 1961, S. 347, Taf. 5, Fig. 1-8; Taf. 6, Fig. 1-9.

Crow Butte, S-Dakota; Hell Creek-Schichten, Mastricht.

Aquilapollenites pulvinus STANLEY

E. A. Stanley, 1961, S. 347-348, Taf. 7, Fig. 1-12.

Crow Butte, S-Dakota; Hell Creek-Schichten, Maastricht. Aquilapollenites reticulatus STANLEY

E. A. Stanley 1961, S. 348, Taf. 8, Fig. 1-12.

Crow Butte, S-Dakota; Hell Creek-Schichten, Maastricht.

Sibirien :

Aquilapollenites trialatus Rouse

N. A. Bolkhovitina, 1959.

Yakutsk SSR, Vilyuy und Tyung; Oberkreide.

Aquilapollenites quadrilobus Rouse

N. A. Bolkhovitina, 1959.

Yakutsk SSR, Vilyuy; Oberkreide.

Aquilapollenites bullatus BolKHovitina

N. A. Bolkhovitina, 1959.

Yakutsk SSR, Vilyuy und Tyung; Oberkreide.

Aquilapollenites aequus BoLKHovitiNA

N. A. Bolkhovitina, 1959.

Yakutsk SSR, Vilyuy; Oberkreide.

Japan :

Aquilapollenites quadrilobus Rouse

S. SATo, 1961, Taf. 1, Fig. 19.

Enbetsu, Nord-Hokkaido; Osoushinai-Schichten, Oberkreide.

Aquilapollenites hakobuchiensis SATo

S. SATo, 1961, S. 91, Taf. 1, Fig. 13-14.

Enbetsu, Nord-Hokkaido; Osoushinai-Schichten, Oberkreide. Aquilapollenites sp.

S. SATo, 1961, S. 92, Taf. 1, Fig. 20.

Enbetsu, Nord-Hokkaido; Osoushinai-Schichten, Oberkreide.

Aquilapollenites triauritus $\mathrm{n}$. sp.

K. TAKahashi (in dieser Arbeit), S. 250-251, Taf. 37, Fig. 1a-c, 2a-c.

Ooyubari, Yubari-Kohlenfeld, Hokkaido; Untere-Hakobuchi-Schichtengruppe, Campan. Aquilapollenites borealis $\mathrm{n}$. sp.

K. Takahashi (in dieser Arbeit), S. 251-252, Taf. 37, Fig. 3a-d, 4a-b.

Ooyubari, Yubari-Kohlenfeld, Hokkaido; Untere Hakobuchi-Schichtengruppe, Campan.

Aquilapollenites matsumotoi n. sp.

K. TAKAhashi (in dieser Arbeit), S. 252-253, Taf. 38, Fig. 1a-b, 3a-b.

Ooyubari, Yubari-Kohlenfeld, Hokkaido; Untere Hakobuchi-Schichtengruppe, Campan. 
Aquilapollenites quadrinus n. sp.

K. Takahashi (in dieser Arbeit) S. 253-254, Taf. 41, Fig. 31a-c.

Ooyubari, Yubari-Kohlenfeld, Hokkaido; Obere Hakobuchi-Schichtengruppe, Maastricht.

Aquilapollenites sp. a.

K. TAkahashI (in dieser Arbeit) S. 254, Taf. 37, Fig. 5a-b.

Ooyubari, Yubari-Kohlenfeld, Hokkaido; Untere Hakobuchi-Schichtengruppe, Campan.

Aquilapollenites sp. b.

K. Takahashi (in dieser Arbeit) S. 254, Taf. 37, Fig. 7a-c.

Ooyubari, Yubari-Kohlenfeld, Hokkaido; Untere Hakobuchi-Schichtengruppe, Campan.

Aquilapollenites sp. c.

K. Takahashi (in dieser Arbeit) S. 254-255, Taf. 37, Fig. 8a-c.

Ooyubari, Yubari-Kohlenfeld, Hokkaido; Untere Hakobuchi-Schichtengruppe, Campan.

Aquilapollenites sp. d.

K. Takahashi (in dieser Arbeit) S. 255, Taf. 38, Fig. 2a-b.

Ooyubari, Yubari-Kohlenfeld, Hokkaido; Untere Hakobuchi-Schichtengruppe, Campan.

? Aquilapollenites sp.

K. Takahashi (in dieser Arbeit) S. 255, Taf. 37, Fig. 6.

Ooyubari, Yubari-Kohlenfeld, Hokkaido; Untere Hakobuchi-Schichtengruppe, Campan.

\section{7) Pentapollenites}

Die Gattung Pentapollenites wurde zuerst von W. KRUTzsch (1957) vorgeschlagen.

Europa :

Pentapollenites (al. Periporopollenites) pentangulus (PfLUG)

P. W. Thomson und H. D. Pflug, 1953, S. 112, Taf. 15, Fig. 62-64.

Borken, Helmstedt, Messel; Eozän.

W. Krutzsch, 1957, S. 520, Taf. 10, Fig. 1-13.

Geiseltal; Eozän ?

Japan :

Pentapollenites yezoensis n. sp.

K. Takahashi (in dieser Arbeit) S. 256, Taf. 36, Fig. 28-31.

Ooyubari, Yubari-Kohlenfeld, Hokkaido; Untere Hakobuchi-Schichtengruppe, Campan.

Laevigatosporites ovatus WILson und WEBSTER, Schizosporis und Aquilapollenites treten nur in den pazifischen Randgebieten, die sich südlich bis nach Australien hin erstrecken, auf und nie in der Oberkreide und dem tieferen Paläogen von Ost-USA und Europa. Von ihnen ist besonders Aquilapollenites sehr interessant. Aquilapollenites wurde nur aus der Oberkreide und dem Paläozän von West-Kanada, West-USA, Sibirien und Nordjapan beschrieben. Im Maastricht von West-USA ist er am häufigsten. Er scheint pollenstratigraphisch sehr wichtig zu sein. Man kann vermuten, daß verhältnismäßige Vorkommen der Sporomorphae in der Provinz der Normapolles-Pollen und in den pazifischen Randgebieten, in denen Normapolles-Pollen nicht auftreten, wahrscheinlich in Mittel-USA erforscht werden könnte.

Die Gattung Phyllocladidites wurde bisher nur in Australien, Neuseeland, 
Nordjapan und der Kerguelen Insel gefunden.

\section{Beschreibung der Sporomorphae}

\section{A. Sporen und Pollen der Hakobuchi-Schichtengruppe.}

\section{Sporites}

Triletes

\section{Formgattung: Cicatricosisporites R. PotoniÉ \& Gelletich}

1933. Cicatricosisporites R. PotoniÉ \& Gelletich, S. 522.

1956. Cicatricosisporites R. Potoníe \& Gelletich, in R. Potonié, S. 47.

Cicatricosisporites cf. dorogensis R. PоT. \& GELL.

Taf. 23, Fig. 1-3.

Die vorliegenden Formen, von denen die Figuren 1 und 2 ungenügend erhalten wurden, sind ca. 45 bis $52 \mu$ groß. Trilete Sporen mit der canaliculat skulpturierten Exine. Muri verlaufen parallel, 1.5 bis $2 \mu$ breit und 3 Stucke in der 7 bis $9.7 \mu$ Breite vorhanden. Die Muri der beiden Oberflächen kreuzen dann einander mehr oder weniger. Umrißlinie gleichmäßig gekerbt. Y-Marke schmal.

Diese Spezies wurde im Kohlenflöz der unteren Hakobuchi-Schichtengruppe von Hatsune-Sawa, Yubari-Kohlenfeld, Hokkaido gefunden.

Cicatricosisporites dorogensis R. Рот. \& GeLl. (1933, S. 522, Taf. 1, Fig. 1; P. W. Thomson \& H. D. Pflug, 1953, S. 48-49, Taf. 1, Fig. 1-12) wurde aus den mitteleuropäischen Paläozän bis Oligozän beschrieben, aber die Grenze der Größe wurde gar nicht gezeigt. Man muß über die Größe durch die Abbildungen urteilen.

F. Thiergart (1940, S. 24, Taf. 6, Fig. 1; Taf. 7, Fig. 25; Taf. 8, Fig. 1, 2, $5,6,9)$ hat dieselben Formen als Mohria-Typ beschrieben und abgebildet.

W. KRUTZSCH (1957) hat einige Abbildungen der dorogensis-Gruppe gezeigt und er (1960) hat Cicatricosisporites cf. cicatricosoides KRUTZSCH und Cicatricosisporites cf. dorogensis R. POT. \& GELL. aus den Schichten der Wilcox-Stufe von Austin, Texas, USA abgebildet.

Morphologisch ähnliche kretazeische Formen, Cicatricosisporites hallei DELCOURT \& SPRUMONT, Cicatricosisporites sewardi DelcourT \& SPRUMONT und Cicatricosisporites mohrioides Delcourt \& SPRumont haben A. Delcourt und G. Sprumont (1955, S. 17, Taf. 1, Fig. E1, Abb. 1; S. 19, Abb. 2; S. 20, Taf. 1, Fig. 2) aus der unteren Kreide von Hainaut, Belgien beschrieben.

G. E. Rouse (1957) hat europäische Spezies Cicatricosisporites dorogensis R. Poт. \& Gell. (S. 362, Taf. 2, Fig. 38, 39) aus den oberkretazeischen OldmanSchichten von Südalberta, Westkanada mitgeteilt und abgebildet. Weiter hat er (1962) Cicatricosisporites intersectus Rouse (S. 197, Taf. 3, Fig. 30, 31) aus 
den eozänen Burrard-Schichten von Third Beach und Terminal Dock (Burrard Insel) und Cicatricosisporites striatus Rouse (S. 197-198, Taf. 4, Fig. 1-2) und Cicatricosisporites sp. (S. 198, Taf. 3, Fig. 29) aus den eozänen BurrardSchichten von Brothers Creek (Burrard Insel) beschrieben.

R. A. Couper (1958) hat Cicatricosisporites dorogensis R. Рот. \& GELL. (S. 138, Taf. 17, Fig. 10-12) aus den Purbeck-Schichten (Wealden), England, Cicatricosisporites brevilaesuratus Couper (S. 136, Taf. 18, Fig. 1-3) aus den kretazeischen Schichten (Wealden), England und Cicatricosisporites dunrobinensis Couper (S. 137, Taf. 17, Fig. 13-15) aus den jurrassischen Schichten (unteres Lias), England beschrieben.

I. C. Cookson (1953) hat Mohrioisporites australiensis Cookson (S. 470, Taf. 2, Fig. 31-34) aus den prätertiären Schichten von Südaustralien beschrieben und weiter hat sie 1954 denselben als Mohriosporites gezeigt. Mohrioisporites und Mohriosporites sind Synonym von Cicatricosisporites.

N. A. Bolkhovitina (1961) hat die fertilen Wedelreste, Pelletieria valdensis SEWARD, die von SEWARD (1913) genannt wurde, als Genotypus der vorliegenden trileten Sporen mit der canaliculaten oder cicatricosen Exinenskulptur angenommen. Aber der Gattungsname Pelletieria ist für Sporae dispersae nicht gültig. Pelletieria mediostriata Bolkhovitina (S. 66, Taf. 19, Fig. 3a, b; Taf. 21, Fig. 1a-c), Pelletieria tersa (KARA-Mursa) (S. 66-67, Taf. 19, Fig. 4a-e; Taf. 21, Fig. 4a-d; Taf. 22, Fig. 1a-s), Pelletieria multabila (Bolк нovitina) (S. 67, Taf. 19, Fig. 5), Pelletieria clara (Bolk hovitina) (S. 67-68, Taf. 19, Fig. 6a, b), Pelletieria volgensis Bolkhovitina (S. 68, Taf. 19, Fig. 7a, b), Pelletieria minutaestriata Bolkhovitina (S. 68, Taf. 20, Fig. 1a-f; Taf. 21, Fig. 3a-d), Pelletieria minor (Bolkhovitina) (S. 68, Taf. 19, Fig. 8; Taf. 22, Fig. 3a-c) und Pelletieria pacifica Bolkhovitina (S. 69, Taf. 22, Fig. 2a-g).

Der Verfasser (1961) hat eine japanische eozäne Art Cicatricosisporites sp. aus den Tooka-Schichten des Miike-Kohlenfeldes, Nordkyushu beschrieben.

Botanische Zugehörigkeit: Schizaeaceae.

Formgattung: Gleicheniidites (Ross) Delcourt \& SPRumonT

1949. Gleicheniidites Ross, S. 31 .

1956. Gleicheniidites (Ross) Delcourt \& Sprumont 1955 in R. Potonié, S. 14, 15.

Gleicheniidites senonicus wurde von N.-E. Ross (1949) aus den oberkretazeischen Schichten (Obersanton oder Untercampan), NE-Bucht des Jvösjon-Sees, Südschweden beschrieben. Er hat keine Beschreibung der Formgattung Gleicheniidites gegeben, aber diese Formgattung ist als monotypisch gültig.

Gleicheniidites marginatus n. sp.

Taf. 23, Fig. 4-17; Taf. 40, Fig. 1.

D i a g n o s e : Trilete Sporen. Ca. $24-37 \mu$ groß. Konkave Äquatorkontur des Exospors. Y-Leisten verlaufen meistens mehr oder weniger wellig oder geradlinig und erreichen den Äquator. Exospor chagrenat, 2 bis $3 \mu$ dick. 
H o l ot y p u s : $28.3 \mu$ groß; Taf. 23, Fig. 7; Präparat GK-V 3142.

Locus typicus: Hatsune-Sawa, Ooyubari, Yubari-Kohlenfeld, Hokkaido.

Stratigraphisches Verhalten: Diese Spezies wurde in den oberkretazeischen Unter-Hakobuchi-Schiten (Campan) von Hatsune-Sawa gefunden.

Ve r g le i c h : N.-E. Ross (1949) hat sehr ähnliche Form, Gleicheniidites senonicus Ross (S. 31, Taf. 1, Fig. 3), aus den oberkretazeischen Tonen von Åsen, Scania, Schweden, beschrieben. Die Exine dieser europäischen Art ist dünner als die der japanischen marginatus-Form. Die Y-Leisten der ersteren verlaufen geradlinig, aber die der letzteren meistens wellig.

Die japanische Spezies ist der mitteleuropäischen tiefsttertiären Spezies, Concavisporites rugulatus Pflug (P. W. Thomson \& H. D. PFlug, 1953, S. 49, Taf. 1, Fig. 19-23), ähnlich, aber die erstere ist chagrenat und die letztere ist glatt.

I. C. Cookson (1953) hat morphologisch ähnliche Form, Gleichenia eircinidites Cookson (S. 464, Taf. 1, Fig. 5-6), aus den tiefsttertiären Kohlen, Südaustralien beschrieben. Die Y-Leisten dieser circinidites-Form sind breiter und stärker als die der betreffenden japanischen Art.

Die von H. Weyland und W. KrIEgER (1953) abgebildeten Formen mit Torus und Disken (Taf. 3, Fig. 19-23) gehören zu Concavisporites, wenn es den Photos nach urteilt. Diese Spezies ist auch der vorliegenden japanischen Form ähnlich.

N. A. Bolkhovitina hat morphologisch ähnliche Spezies, Gleichenia stellata Bolk hovitina (1953, S. 23, Taf. 2, Fig. 8-10) aus den unterkretazeischen Tonen von West-Kuzakhstan an der Nordküste von Aral-See beschrieben.

Botanische Zugehörigkeit: Gleicheniaceae.

\section{Formgattung: Concavisporites PFLUG}

1953. Concavisporites Pflug in P. W. Thomson \& H. D. Pflug, S. 49.

\section{Concavisporites macellus $\mathrm{n}$. $\mathrm{sp}$.}

Taf. 23, Fig. 18, 19.

D i a g nos e : Trilete Sporen. $30-31.3 \mu$ groß. Exospor mit konkaver Äquatorkontur, sehr dünn, chagrenat bis fein rugulat. Torus flächig, gegen die Y-Marke nicht abgesetzt. Y-Leisten stark, geradlinig verlaufend, den Äquator nicht erreichend.

H o loty pus : $31.3 \mu$ groß; Taf. 23, Fig. 18; Präparat GK-V 3131.

L ocus typicus: Hatsune-Sawa, Ooyubari, Yubari-Kohlenfeld, Hokkaido.

Stratigraphisches Verhalten: Diese Spezies wurde bisher nur in den oberkretazeischen Unter-Hakobuchi-Schichten (Campan) von HatsuneSawa, Yubari-Kohlenfeld, Hokkaido gefunden. 
Verglei ch : Die macellus-Form ist der mitteleuropäischen oberkretazeischen bis unterpaläogenen Spezies, Concavisporites exiguus PFLUG (P. W. Thomson \& H. D. Pflug, 1953, S. 50, Taf. 1, Fig. 44-46), ähnlich. Die mitteleuropäische Spezies hat intragranulate bis intrareticulate Struktur und der Torus ist wulstig und scharf abgesetzt.

G. E. Rouse (1957) hat morphologisch ähnliche Formen, Cibotiumsporites concavus Rouse (S. 354, Taf. 1, Fig. 36-37) aus den oberkretazeischen ComoxSchichten (Campan) von der Vancouver Insel und Gleichenia concavisporites Rouse (S. 363, Taf. 2, Fig. 36, 48; Taf. 3, Fig. 49) aus den oberkretazeischen Oldman-Schichten (Campan) von Südalberta beschrieben. Er hat neue Gattung Cibotiumsporites festgesetzt. Die concavisporites-Form ist glatt.

R. A. Couper $(1953,1958)$ hat Cyathidites australis Couper aus den jurassischen Ohika-Schichten von Buller George, Neuseeland und Cyathidites minor Couper aus den jurassischen und unterkretazeischen Schichten von England beschrieben. Die Gattung Cyathidites ist morphologisch der anderen Gattung Concavisporites ähnlich, aber die letztere besitzt zusätzlich Kyrtome (=Tori). Nach R. A. COUPER ist Cyathidites australis der Spore von Cladophlebis lobifolia und Cyathidites minor der von Coniopteris hymenophylloides ähnlich.

Botanische Zugehörigkeit: Vieleicht Gleicheniaceae.

\section{Concavisporites sp. a}

Taf. 23, Fig. 20.

Die vorliegende Form wurde bisher nur im oberkretazeischen Kohlenflöz (Campan) von Hatsune-Sawa, Yubari-Kohlenfeld, Hokkaido gefunden. Nur ein Korn wurde gefunden.

Die Figur 20 ist trilete Spore und $37.5 \mu$ groß. Konkave Äquatorkontur des Exospors. Exospor dünn, schwach glatt. Torus wulstig, gegen die Y-Marke scharf abgesetzt, mit Disken. Y-Leisten verlaufen geradlinig und erreichen den Äquator.

Diese betreffende Spezies ist der mitteleuropäischen tieftertiären Art, Concavisporites rugulatus Pflug (P. W. Thomson \& H. D. Pflug, 1953, S. 49, Taf. 1, Fig. 19-23), ähnlich.

Botanische Zugehörigkeit: Vielleicht Gleicheniaceae.

\section{Concavisporites $\mathrm{sp} . \mathrm{b}$ \\ Taf. 40, Fig. 3a, b.}

Die vorliegende trilete Spore ist klein, ca. $21 \mu$ groß. Konkave Äquatorkontur des Exospors. Exospor dünn, chagrenat. Torus wulstig, gegen die Y-Marke scharf abgesetzt, mit Disken(?). Y-Leisten verlaufen geradlinig und erreichen keinen Äquator.

Die Spezies b wurde nur in der oberen Hakobuchi-Schichtengruppe (Feinsandstein, Maastricht), bei der Talspere von Ooyubari, Yubari-Kohlenfeld, Hokkaido gefunden. 
Botanische Zugehörigkeit: Vielleicht Gleicheniaceae.

\section{? Concavisporites sp.}

Taf. 40, Fig. 2.

Diese trilete Spore gehört zu Concavisporites zweifelhaft, da sie nicht so gut erhalten ist. Sie ist ca. $47 \mu$ groß. Es ist unklar, ob der Torus vorhanden ist oder nicht. Y-Leisten verlaufen geradlinig und erreichen keinen Äquator.

Die betreffende Art wurde bisher nur in der oberen Hakobuchi-Schichtengruppe (Feinsandstein, Maastricht), bei der Talspere von Ooyubari, YubariKohlenfeld, Hokkaido gefunden.

Botanische Zugehörigkeit: fraglich.

\section{Formgattung: Divisisporites PFLUG}

1953. Divisisporites Pflug, S. 51.

? Divisisporites sp.

Taf. 23, Fig. 21a, b.

Die vorliegende Form wurde bisher nur im oberkretazeischen Kohlenflöz (Campan) von Hatsune-Sawa, Yubari-Kohlenfeld, Hokkaido gefunden. Ein Teil dieses Korns ist zerstört.

Diese Form ist $30 \mu$ groß. Exospor $0.8 \mu$ dick, chagrenat. Äquatorkontur rundlich. Y-Spaltmarke schließt einen stumpfen Spaltwinkel ein. Y-Leisten erreichen den $\ddot{\text { Aquator nicht. }}$

Botanische Zugehörigkeit: fraglich.

\section{Formgattung: Verrucosisporites (IBRAHIM) R. PotoniÉ \& KREMP}

1933. Verrucosisporites IBRAHIM, S. 24.

1955. Verrucosisporites (IBRAhim) Potoní́ \& KREMP, S. 65.

Verrucosisporites permirus n. sp.

Taf. 23, Fig. 22-24.

Di a g n o s e : Trilete Sporen. 25.4-30 $\mu$ groß. Äquatorkontur dreieckig mit stark konvexen Seiten und abgerundeten Ecken. Y-Leisten schmal, den Äquator nicht erreichend, ohne Tori, Disken, selten aufgespalten. Exospor $1.8 \mu$ dick, groß rugulat oder papillat skulpturiert.

H o l o ty p u s : $30 \mu$ groß; Taf. 23, Fig. 22 ; Präparat GK-V 3172.

Locus typicus: Hatsune-Sawa, Ooyubari, Yubari-Kohlenfeld, Hokkaido.

Stratigraphisches Verhalten: Man konnte bisher diese neue Spezies nur in den oberkretazeischen Unter-Hakobuchi-Schichten (Campan) finden. 
Vergle i c h: I. C. Cookson (1953) hat Sphagnites australis (Cookson) forma crassa Cookson (S. 464, Taf. 1, Fig. 2-4; Taf. 2, Fig. 24) aus den unterpaläogenen Kohlen von Comaum, Südaustralien beschrieben. Diese australische Form ist etwas größer als die permirus-Form und ihr Exospor ist glatt oder schwach uneben und ca. $3-\mathbf{5} \mu$ dick.

N. A. BoLk hovitina (1961) hat scheinbar morphologisch ähnliche Form, Lygodium gibberulum KARA-MURSA var. gibberula KARA-MURSA (S. 90, Taf. 28, Fig. 4a, b; Taf. 36, Fig. 3a-k) aus den unterkretazeischen Schichten in den verschiedenen Gegenden von Rußland abgebildet und beschrieben. In Rußland wurde Lygodium gibberulum KARA-MURSA bisher in den oberjurassischen bis bis oberkretazeischen Schichten gefunden. Die vorliegende permirus-Form ist vor allen den Figuren 3a und 3d der Tafel 36 von N. A. Bolk hovitina (1961) morphologisch ähnlich. Lygodium gibberulum KARA-MURSA var. gibberula KARAMURSA ist meistens schwach konvex-dreieckig und das Exospor ist gröber als das der permirus-Form skulpturiert.

S. MANUM (1962) hat morphologisch sehr ähnliche Formen, Verrucosisporites pulvinulatoides MANUM (S. 28, Taf. 3, Fig. 5, 6) und Verrucosisporites pulvinulatus MANUM (S. 27-28, Taf. 3, Fig. 7-10), aus den tertiären Schichten von Spitzbergen beschrieben. Besonders ist die pulvinulatus-Form der japanischen Spezies sehr ähnlich, aber die rundlichen Flecke der ersteren sind größer als die der letzteren und die Exine der ersteren ist etwas dünner als die der letzteren.

Botanische Zugehörigkeit: Lygodium ? oder Sphagnum ?

\section{Verrucosisporites sp. \\ Taf. 40, Fig. 10.}

Die vorliegende Form ist trilete Spore und ca. $27 \mu$ groß. Äquatorkontur dreieckig mit stark konvexen Seiten und abgerundeten Ecken. Y-Leisten wulstig, $1.8 \mu$ breit, den Äquator fast erreichend. Umrißlinie des Exospors unduliert, $\pm 1 \mu$ hoch.

Sie wurde im Sandstein der oberen Hakobuchi-Schichtengruppe (Maastricht) bei der Talspere von Ooyubari, Yubari-Kohlenfeld, Hokkaido gefunden.

Botanische Zugehörigkeit: fraglich.

\section{Formgattung: Stereisporites PFLUG}

1953. Stereisporites Pflug in P. W. Thomson \& H. D. Pflug, S. 53. 1956. Sphagnumsporites RAATZ ex R. PotoniE, S. 17.

R. PotoniÉ (1956, S. 17) hat die Gattung Sphagnumsporites von RAATZ (1937, S. 9) als gültig behandelt und die Gattung Stereisporites von PfLUG (P. W. Thomson \& H. D. Pflug, 1953, S. 53) für ein Synonym angesehen. G. V. RAATZ (1937) hat die neue Gattung Sphagnumsporites für die Art, Sporites stereoides, die von R. POTONIÉ \& H. Venitz (1934) erstmals beschrieben wurde, gegeben, aber dabei hat er keine Beschreibung der Gattung Sphagnumsporites 
beigefügt, sondern nur die Beschreibung der Spezies gegeben. Deshalb ist die Gattung Sphagnumsporites ein Synonym von Stereisporites, wie S. MANUM (1962, S. 26) behauptet hat.

Stereisporites limbatus n. sp.

Taf. 24, Fig. 1-12.

D i a $\mathrm{g}$ n o s e : Trilete Sporen. 20.6-34.3 $\mu$ groß. Äquatorkontur dreieckig mit konvexen Seiten und abgerundeten Ecken. Y-Leisten schwach, schmal den Äquator nicht erreichend, ohne Tori, Disken und Aufspaltung. Exospor 1.3 bis $2.3 \mu$ dick, meistens mit unregelmäßiger brauner Umrißlinie (2 bis $3.4 \mu$ dick), schwach chagrenat bis schwach glatt.

H o loty pus : $26.6 \mu$ groß; Taf. 24, Fig. 4a, b; Präparat GK-V 3132.

Locus typicus: Hatsune-Sawa, Ooyubari, Yubari-Kohlenfeld, Hokkaido.

Stratigraphisches Verhalten : Diese Spezies tritt bisher nur in den oberkretazeischen Unter-Hakobuchi-Schichten (Campan) von HatsuneSawa, Yubari-Kohlenfeld auf.

V e r g l e i c h : Die limbatus-Form ist den mitteleuropäischen tertiären bis oberkretazeischen Spezies, Stereisporites psilatus (Ross) PFLUG (P. W. THoMson \& H. D. Pflug, 1953, S. 53, Taf. 1, Fig. 75-80; H. WeYland \& W. Krieger, 1953, S. 10, Taf. 3, Fig. 29-30), Stereisporites stereoides (R. PoT. \& Ven.) Pflug (Sporites stereoides R. Ротоní́ \& Venitz, 1934, S. 11, Taf. 1, Fig. 4-5; P. W. Thomson \& H. D. Pflug, 1953, S. 53, Taf. 1, Fig. 64-73) und Streisporites megastereoides Pflug (P. W. Thomson \& H. D. Pflug, 1953, S. 53, Taf. 1, Fig. 74), ähnlich. Die Exine der psilatus-Form ist etwas dicker als die der limbatus-Form. Die Y-Marke der stereoides-Form erreicht den Äquator und die Exine ist etwas dünner als die der limbatus-Form. Die megastereoides-Form ist dicker und größer. Ihre Y-Leisten erreichen den Äquator.

Sphagnum antiquasporites WILSON und WEBSTER wurde von L. R. WILSON und R. M. Webster (1946, S. 273, Fig. 2) aus den paläozänen Fort Union Schichten von Montana, USA, erstmals beschrieben und später von G. E. RousE (1957, S. 353, Taf. 1, Fig. 32, 33) aus den oberkretazeischen Comox-Schichten (Campan) von der Vancouver Insel abgebildet. Das Exospor dieser Form ist dünner als das der limbatus-Form. G. E. Rouse (1957) hat Stenozonotriletes simplex Naumova (G. E. Rouse, 1957, S. 354, Taf. 1, Fig. 40-41; Taf. 2, Fig. 46-47) aus den oberkretazeischen Comox- und Oldman-Schichten mitgeteilt.

I. C. Cookson hat Trilites australis Cookson (1947, S. 136, Taf. 15, Fig. 58-59) aus den tertiären Braunkohlen und Sandsteinen von der Kerguelen Insel, Sphagnites australis (Cookson) forma parva Cookson (1953, S. 464, Taf. 1, Fig. 1; Taf. 2, Fig. 25-26) und Sphagnites australis (Cookson) forma crassa Cookson (1953, S. 464, Taf. 1, Fig. 2-4; Taf. 2, Fig. 24) aus den tiefsttertiären Kohlen von Südaustralien beschrieben. Die australis forma crassa Form ist morphologisch der limbatus-Form mehr ähnlich, aber das Exospor der ersteren ist dicker als das der letzteren. 
Botanische Zugehörigkeit: Wahrscheinlich Sphagnum.

Stereisporites grossus n. sp.

Taf. 24, Fig. 13-15.

D i a g n o s e : Trilete Sporen. Ca. 40-47.4 $\mu$ groß. Äquatorkontur dreieckig mit konvexen Seiten und abgerundeten Ecken (viel mehr elliptisch). YLeisten schmal, den Äquator nicht erreichend, ohne Tori, Disken, Aufspaltung. Exospor 3.8 bis $4.3 \mu$ dick, mit brauner Umrißlinie, schwach changrenat oder zuweilen papillat skulpturiert.

H o l o ty p u s : $42.5 \mu$ groß; Taf. 24, Fig. 13; Präparat GK-V 3172.

Locus typicus: Hatsune-Sawa, Ooyubari, Yubari-Kohlenfeld, Hokkaido.

Stratigraphisches Verhalten: Diese Spezies wurde bisher nur in den oberkretazeischen Unter-Hakobuchi-Schichten (Campan) von Hatsune-Sawa, Yubari-Kohlenfeld gefunden.

Vergle i ch: Die große Spezies von Mitteleuropa, Stereisporites megastereoides Pflug (P. W. Thomson \& H. D. Pflug, 1953, S. 53, Taf. 1, Fig. 74), ist über $30 \mu$ groß. Ihre Y-Leisten erreichen den Äquator und ihre Äquatorkontur ist dreieckig.

I. C. Cookson (1947) hat morphologisch ähnliche Spezies, Trilites (Cyatheacidites) annulata CooksoN (S. 136, Taf. 15, Fig. 53-55), aus den tertiären Braunkohlen und Sandsteinen von „Waterfall Gorge“, Kerguelen Insel beschrieben. Diese Art ist größer als die vorliegende Spezies und hat viel dickeren Ring am Rand.

Botanische Zugehörigkeit: Wahrscheinlich Sphagnum.

Stereisporites pseurlostereoides $\mathrm{n}$. sp.

Taf. 24, Fig. 16-23.

D i a g n o s e : Trilete Sporen. 26.7-34.5 $\mu$ groß. Äquatorkontur dreieckig mit konvexen Seiten und abgerundeten Ecken. Y-Leisten verhältnismäßig stark, schmal, bis die Nähe des Äquators erreichend, ohne Tori, Disken und Aufspaltung. Exospor unter $2 \mu(0.7$ bis $1.9 \mu)$ dick, oft mit unregelmäßiger schwacher Umrißlinie, schwach chagrenat bis schwach glatt.

H o l o t y p u s : $31.4 \mu$ groß; Taf. 24, Fig. 16; Präparat GK-V 3132.

Locus typicus: Hatsune-Sawa, Ooyubari, Yubari-Kohlenfeld, Hokkaido.

Stratigraphisches Verhalten: Die Spezies wurde bisher in den oberkretazeischen Unter-Hakobuchi-Schichten (Campan) von Hatsune-Sawa, Yubari-Kohlenfeld gefunden.

Ve r g l e i c h : Die betreffende Spezies ist der limbatus-Form ähnlich, aber die letztere besitzt die braune dicke Umrißlinie und die Y-Leisten der ersteren ist länger und etwas stärker als die der letzteren.

Die pseurlostereoides-Form ist den mitteleuropäischen tertiären bis ober- 
kretazeischen Spezies, Stereisporites psilatus (Ross) PfLug (P. W. Thomson \& H. D. Pflug, 1953, S. 53, Taf. 1, Fig. 75-80 ; H. Weyland \& W. Krieger, 1953, S. 10, Taf. 3, Fig. 29-30), Stereisporites stereoides (R. Pot. \& Ven.) Pfeug (Sporites stereoides R. PotoniÉ \& Venitz, 1934, S. 11, Fig. 4-5; P. W. Thomson \& H. D. Pflug, 1953, Taf. 1, Fig. 64-73) und Stereisporites megastereoides Pflug (P. W. Thomson \& H. D. Pflug, 1953, S. 53, Taf. 1, Fig. 74), ähnlich. Die Y-Leisten der stereoides-Form erreichen den Äquator, aber die der pseudostereoirles-Form nicht. Das Exospor der megastereoides-Form ist dicker als das der pseudostereoides-Form.

L. R. Wilson \& R. M. Webster (1946) und G. E. Rouse (1957) haben Sphagnum antiquasporites Wilson \& WebsteR (L. R. Wilson \& R. M. Webster, 1946, S. 273, Fig. 2; G. E. Rouse, 1957, S. 353, Taf. 1, Fig. 32-33) aus den Fort Union Schichten von Montana und aus den Comox-Schichten von der Vancouver. Insel beschrieben und abgebildet. Diese Spezies ist kleiner als die betreffende japanische Form und ihre Y-Leisten sind kürzer und erreichen den Äquator.

Morphologisch nahestehende Form Trilites australis Cookson (1947, S. 136, Taf. 15, Fig. 58-59), die später (1953) als Sphagnites australis emendiert wurde, hat I. C. Cookson aus den tertiären Braunkohlen und Sandsteinen von der Kerguelen Insel beschrieben. Die australis-Form ist 34-45 $\mu$ groß und ihr Exospor ist dicker als das der pseudostereoides-Form.

A. F. Flonova (1960) hat Stenozonotriletes pumilus Naumova (S. 35, Taf. 4, Fig. 16-17) aus den unterpaläogenen Schichten von Prov. Moskau beschrieben. Diese Spezies ist kleiner als die pseudostereoides-Form und ihr Exospor ist dicker als das der japanischen Form.

Botanische Zugehörigkeit: Wahrscheinlich Sphagnum.

Stereisporites ? tenuiculus $\mathrm{n} . \mathrm{sp}$.

Taf. 24, Fig. 24, 25.

D i a g no s e : Trilete Sporen. $37-40 \mu$ groß. Äquatorkontur dreieckig mit konvexen Seiten und mehr oder weniger abgerundeten Ecken. Y-Leisten verhältnismäßig stark, den Äquator nicht erreichend, ohne Tori, Disken und Aufspaltung. Exospor dünn, schwach glatt. Oft verfaltet.

H o loty p u s : $37 \mu$ groß; Taf. 24, Fig. 25; Präparat GK-V 3132.

L ocus typicus: Hatsune-Sawa, Ooyubari, Yubari-Kohlenfeld, Hokkaido.

Stratigraphisches Verhalten: Diese Spezies wurde bisher nur in den oberkretazeischen Unter-Hakobuchi-Schichten (Campan) von HatsuneSawa, Yubari-Kohlenfeld gefunden.

V e r g l e i c h: Die betreffende Spezies ist den japanischen tertiären Arten, Stereisporites ? sp. (Kasuya-Form) (K. TAKAHASHI, 1961, S. 281-282, Taf. 13, Fig. 4) und Stereisporites sp. (Ideyama-Form) (K. TAKAHASHI, 1961, S. 282, Taf. 13, Fig. 5) scheinbar ähnlich. Zwei letztere Formen haben dickere Exinen als die tenuiculus-Form. 
I. C. Cookson (1947) hat morphographisch ähnliche Art, Trilites australis Cookson (S. 136, Taf. 15, Fig. 58-59), die später (1953) als Sphagnites australis emendiert wurde, beschrieben. Diese Form besitzt ca. $2.5 \mu$ dicke Wand, aber die tenuiculus-Form hat nur dünne Wand.

Morphologisch ähnliche Spezies Sphagnum antiquasporites WiLsoN \& Webster haben L. R. Wilson und R. M. Webster (1946, S. 273, Fig. 2) aus den paläogenen Fort Union Schichten von Montana, USA, erstmals beschrieben und später hat G. E. Rouse (1957, S. 353, Taf. 1, Fig. 32-33) dieselbe aus den oberkretazeischen Comox-Schichten (Campan) von der Vancouver Insel (WestKanada) mitgeteilt. Die antiquasporites-Form ist viel kleiner.

Botanische Zugehörigkeit: Sphagnum ?

Formgattung: Laevigatisporites (IBRAhim) R. Potonié \& KREmP

1933. Laevigatisporites IBRAHIM

1953. Laevigatisporites (BenNie \& Kidston) Ibrahim in Thomson \& PFlug, S. 53.

1955. Laevigatisporites (IBRAhim, non BenNie \& Kidston) ex Potonié \& KRemp, S. 51-52.

Laevigatisporites magnus $\mathrm{n} . \mathrm{sp}$.

Taf. 25, Fig. 5a-b, 6.

D i a g n o s e : Trilete Sporen. 53.6-61.5 $\mu$ groß. Äquatorkontur dreieckig mit geradlinigen oder sehr schwach konvexen Seiten und abgerundeten Ecken. Y-Leisten legen ungefähr $3 / 4$ des Weges zum Äquator zurück, spalten oft spitzwinkelig auf. Exospor an den Ecken 2.1 bis $3.7 \mu$ dick, an den Seitenmitten 1.3 bis $2.7 \mu$ dick, glatt.

H o loty p u s : $53.6 \mu$ groß; Taf. 25, Fig. 5a, b; Präparat GK-V 3133.

Locus typicus: Hatsune-Sawa, Ooyubari, Yubari-Kohlenfeld, Hokkaido.

Stratigraphisches Verhalten: Diese Spezies wurde bisher nur in den oberkretazeischen Unter-Hakobuchi-Schichten (Campan) von HatsuneSawa, Yubari-Kohlenfeld gefunden.

Vergleich: Die magnus-Form ist den mitteleuropäischen tertiären Sporen, Laevigatisporites neddeni R. Рот. regularis PfLUG (P. W. Thomson \& H. D. Pflug, 1953, S. 54, Taf. 1, Fig. 85-92; Taf. 2, Fig. 1), Laevigatisporites neddeni R. Pot. irregularis Pflug (P. W. Thomson \& H. D. Pflug, 1953, S. 54, Taf. 2, Fig. 2-7), Laevigatisporites neddeni R. Pot. torus PfLUG (P. W. Thomson \& H. D. Pflug, 1953, S. 54, Taf. 2, Fig. 8-17) und Laevigatisporites pseudomaximus Thomson \& Pflug (1953, S. 54, Taf. 2, Fig. 18-23), ähnlich. Die neddeni-Form ist kleiner als die magnus-Form. Die pseudomaximus-Form hat zweischichtiges Exospor und mehr rundlich als die magnus-Form.

H. D. Pflug (1959) hat ähnliche Spore, Laevigatisporites pseudoadriennis Pflug (S. 149, Taf. 13, Fig. 2, 4) aus den jungtertiären und altquartären Schichten von Heidarspordsá (Island) beschrieben. Das Exospor dieser Spezies ist dünner als das der magnus-Form. Die pseudoadriennis-Form ist mehr 
rundlich.

Trilites (Alsophilidites) kerguelensis Cookson (1947, S. 136, Taf. 16, Fig. 69) ist der magnus-Form scheinbar ähnlich. Die Y-Leisten der ersteren erreichen den Äquator, aber die der letzteren nicht.

G. E. Rouse (1957) hat die ähnlichen Arten Sporites nerldeni R. PotoniÉ (G. E. Rouse, 1957, S. 356-357, Taf. 1, Fig. 38) aus den Comox-Schichten (Campan) von der Vancouver Insel und Hymenophyllumsporites deltoida Rouse (1957, S. 363, Taf. 3, Fig. 54-56) aus den Oldman-Schichten (Campan) von Südalberta beschrieben. Er hat die neue Formgattung Hymenophyllumsporites wegen der Vereinigung der Sporen, die mit Hymenophyllum in engerer Beziehung stehen, geschaffen. Später hat er Deltoidospora psilostoma RousE (1959, S. 311, Taf. 2, Fig. 7-8) aus den Kootenay-Schichten von Südost British Columbia beschrieben. Die Formgattung Deltoidospora wurde von E. L. MiNer (1935) geschaffen, aber die Beschreibung ist sehr einfach und ungenügend.

Botanische Zugehörigkeit: Wahrscheinlich Lygodium.

\section{Laevigatisporites hokkaidoensis n. sp.}

Taf. 25, Fig. 1-4.

D i a g n o s e : Trilete Sporen. 34.7-38.4 $\mu$ groß. Äquatorkontur dreieckig mit konvexen Seiten und mehr oder weniger abgerundeten Ecken. Y-Leisten schmal, zuweilen wulstig $(0.7 \mu \pm$ breit), den Äquator erreichend. Exospor 1.5 bis $2.3 \mu$ dick (einschichtig), glatt. Häufig sekundär verfaltet.

H o l o ty p u s : $38.4 \mu$ groß; Taf. 25, Fig. 2; Präparat GK-V 3143.

Locus typicus: Hatsune-Sawa, Ooyubari, Yubari-Kohlenfeld, Hokkaido.

Stratigraphisches Verhalten: Diese Art wurde bisher nur in den oberkretazeischen Unter-Hakobuchi-Schichten (Campan) von HatsuneSawa, Yubari-Kohlenfeld gefunden.

Vergleich: R. PotoniÉ hat früher Sporonites neddeni R. PotoniÉ (1931, S. 332, Tab. 1, Fig. 5) und Sporites neddeni R. Potoní́ (1934, S. 36, Taf. 1, Fig. 12) abgebildet und beschrieben. Später haben P. W. THomson und H. D. Pflug die neddeni-Form von R. Potoní́ emendiert und weiter sie in die drei Subspezies geteilt, nämlich Laevigatisporites neddeni (R. Рот.) THomson \& Pflug regularis Pflug (1953, S. 54, Taf. 1, Fig. 85-92; Taf. 2, Fig. 1), Laevigatisporites neddeni (R. Pот.) Thomson \& Pflug irregularis Pflug (1953, S. 54, Taf. 2, Fig. 2-7) und Laevigatisporites neddeni (R. Рот.) THomson \& Pflug torus Pflug (1953, S. 54, Taf. 2, Fig. 8-17). Die hokkaidoensis-Form ist dieser neddeni-Form sehr ähnlich, aber die letztere ist deutlich zweischichtig.

H. Weyland und W. KrIEger haben Laevigatisporites neddeni (R. РоT.) (1953, S. 10, Taf. 4, Fig. 10, 15) aus den mittleren senonischen Basistonen von Aachen (Deutschland) abgebildet.

E. L. MiNeR (1935) hat Deltoidospora cascadensis Miner (S. 618, Taf. 24, Fig. 9-12) aus den unterkretazeischen Kootenai-Schichten im Great Falls Kohlen- 
feld, Montana, USA beschrieben. Die Y-Leisten der cascadensis-Form erreichen nicht den Äquator.

Botanische Zugehörigkeit: Cyatheaceae ? oder Schizaeaceae ?

\author{
Formgattung: Punctatisporites (IBRAHIM) R. PotoniÉ \& KREMP \\ 1933. Punctatisporites IBRAHIM, S. 21. \\ 1953. Punctatisporites IBRAhim in Thomson \& Pflug, S. 57. \\ 1955. Punctatisporites (IBRAHIM) ex Potonit \& KrEMP, S. 41-42.
}

Punctatisporites punctulatus n. sp.

Taf. 25, Fig. 7-15; Taf. 27, Fig. 1.

D i a g n o s e : Trilete Sporen. Ca. 23.6-42 $\mu$ groß. Äquatorkontur dreieckig mit schwach konvexen Seiten und abgerundeten Ecken. Y-Strahlen deutlich, den Äquator ganz oder fast erreichend. Exospor sehr dünn, meistens punctat. Oft sekundär gefältet.

H ol o ty pu s : $36 \mu$ groß; Taf. 25, Fig. 7; Präparat GK-V 3131.

Locus typicus: Hatsune-Sawa, Ooyubari, Yubari-Kohlenfeld, Hokkaido.

Stratigraphisches Verhalten : Man konnte diese Spezies nur in den oberkretazeischen Unter-Hakobuchi-Schichten von Hatsune-Sawa, YubariKohlenfeld finden.

Vergle i ch: Diese vorliegende Spezies ist der mitteleuropäischen paläogenen Art, Punctatisporites punctatus Pflug (P. W. Thomson \& H. D. Pflug, 1953, S. 57, Taf. 2, Fig. 63-70), morphologisch ähnlich, aber die Exine der letzteren ist deutlich zweischichtig.

Morphologisch ähnliche Form, Deltoidospora rhytisma Rouse (1957, S. 355356, Taf. 1, Fig. 17, 34-35) hat G. E. Rouse aus den oberkretazeischen ComoxSchichten (Campan) von der Vancouver Insel beschrieben. Besonders ist die Figur 35 der Tafel 1 der punctulatus-Form näher ähnlich.

Die betreffende Spezies ist der japanischen anderen neuen Art Rugulatisporites parvirugulatus (in dieser Arbeit, Taf. 26, Fig. 6-8) morphologisch ähnlich. Die Exine der letzteren ist fein rugulat.

Botanische Zugehörigkeit: fraglich.

Punctatisporites hatsunesawaensis n. sp.

Taf. 25, Fig. 16, 17.

D i a g n os e : Trilete Sporen. Ca. 31-33.5 $\mu$ groß. Äquatorkontur dreieckig mit konvexen Seiten und abgerundeten Ecken. Y-Leisten schmal, den Äquator ganz oder nahezu erreichend, selten wulstig (1.2 $\mu$ breit). Exospor 1 bis $2 \mu$ dick, chagrenat.

H o l o ty pus : $33.5 \mu$ groß; Taf. 25, Fig. 17; Präparat GK-V 3142.

Locus typicus: Hatsune-Sawa, Ooyubari, Yubari-Kohlenfeld, Hokkaido. 
Stratigraphisches Verhalten: Die hatsunesawaensis-Form wurde bisher nur in den oberkretazeischen Unter-Hakobuchi-Schichten (Campan) von Hatsune-Sawa, Yubari-Kohlenfeld gefunden.

Vergle i ch: Die betreffende Spezies ist der Art, Punctatisporites punctulatus TAKahashi (In dieser Arbeit, Taf. 25, Fig. 7-15) ähnlich. Die letztere ist fein punctat skulpturiert und ihr Exospor ist sehr dünn.

Die hatsunesawaensis-Form ist den Spezies, Stereisporites ? tenuiculus TAKahashi (Taf. 24, Fig. 24-25) und Laevigatisporites hokkaidoensis TAKAHASHI (Taf. 25, Fig. 1-4) ähnlich, aber die erstere weicht von zwei letzteren in einigen Zügen ab.

Botanische Zugehörigkeit: fraglich.

\section{Punctatisporites sp.}

Taf. 26, Fig. 1.

Trilete Spore. $43 \mu$ groß. Äquatorkontur rundlich. Y-Leisten schmal, verhältnismäßig kurz. Exospor dünn, punctat bis chagrenat, verfaltet.

Nur ein Korn dieser Form wurde im oberkretazeischen Kohlenflöz der unteren Hakobuchi-Schichtengruppe (Campan) von Hatsune-Sawa, Yubari-Kohlenfeld gefunden.

Botanische Zugehörigkeit: fraglich.

\section{Formgattung: Dictyophyllinites COUPER}

1958. Dictyophyllidites Couper, S. 140.

1961. Fasciatisporites SATO, S. 85 .

S. SAto (1961) hat die neue Formgattung Fasciatisporites geschaffen, aber sie besitzt gleiche Eigentümlichkeiten mit der Formgattung Dictyophyllidites, die schon von R. A. Couper (1958) gegeben wurde. Deshalb verschwindet, entsprechend dem Internationalen Code, der Name Fasciatisporites in der Gattung Dictyophyllidites.

Dictyophyllidites divergens (SATo) n. comb.

Taf. 25, Fig. 18, 19.

1961. Fasciatisporites divergens, S. SATo, S. 85-86, Taf. 1, Fig. 6-10.

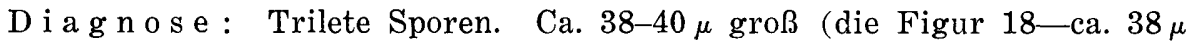
groß; die Figur 19-40 $\mu$ groß). Äquatorkontur dreieckig mit konvexen Seiten und mehr oder weniger abgerundeten Ecken. Y-Leisten oft wulstig ( $1 \mu$ breit),

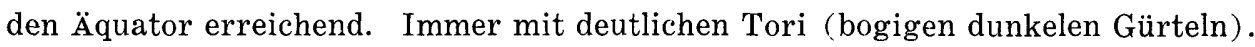
Exospor dünn, $\pm 0.5 \mu$ dick, schwach chagrenat bis fein punctat.

Verg l e i c h : R. A. Couper (1958) hat die ähnliche Art, Dictyophyllidites harrisii Couper (S. 140, Taf. 21, Fig. 5-6), aus den jurassischen Schichten von Yorkshire (England) beschrieben. Die Exine der harrisii-Form ist glatt und 
zwei- bis viermal dicker als die der japanischen Form.

Die paläozoische Art, Leiotriletes adnatoides Pot. \& KREMP (1955, S. 38, Taf. 11, Fig. 112-115), ist der vorliegenden Spezies ähnlich, aber bei der ersteren erscheint Infrapunktierung im näheren Bereich der Y-Strahlen deutlicher als in den übrigen Bereichen der Exinen und bei der letzteren erscheinen dunkele Zonen auf den Flächen der pyramidenförmigen Gestalt bogig.

Vorkommen: Hatsune-Sawa (untere Hakobuchi-Schichtengruppe, Campan); bei Enbetsu (Osoushinai-Schichten).

Botanische Zugehörigkeit: Nach R. A. Couper ist die Spore Dictyophyllidites der Spore von Dictyophyllum ähnlich.

\section{Formgattung: Corrugatisporites (THomson \& PFLUG) WEYLAND \& GREIFELD}

1953. Corrugatisporites Ibrahim ex Thomson \& Pflug, S. 55.

1956. Corrugatisporites (Thomson \& Pflug 1953, non Ibrahim) Weyland \& GReifeld in R. Potoní, S. 41.

Corrugatisporites cf. solidus (R. Рот.) Thomson \& PFLUG

Taf. 40, Fig. 5.

Die vorliegende Form ist nicht gut erhalten. Sie ist trilete Spore und $35.6 \mu$ groß. Äquatorkontur dreieckig mit abgerundeten Ecken. Über $2 \mu$ hohe und an der Basis über $2 \mu$ breite Warzen, z. T. im Grundriß verlängert, sind rugulat angeordnet. Y-Leisten schmal, den Äquator nicht erreichend.

Sie wurde im Sandstein der oberen Hakobuchi-Schichtengruppe (Maastricht), bei der Talspere von Ooyubari, Yubari-Kohlenfeld, Hokkaido gefunden.

R. PotoniÉ (1934) hat Sporites solidus R. PотоNiÉ (S. 42, Tab. 1, Fig. 35) aus den eozänen Braunkohlen, Geiseltal (Deutschland), erstmals beschrieben. Später haben P. W. Thomson \& H. D. Pflug (1953, S. 55) und H. WeYland \& G. GREIFELd (1953, S. 42) IbRAHIM als Autor der Gattung Corrugatisporites bezeichnet. Dabei haben H. WeYland und G. GReIfeld in die Gattung Corrugatisporites zwei neue Formen gebracht. R. PotoniÉ (1956, S. 41) hat geschrieben, daß als Genotyp der Gattung Corrugatisporites die Spezies toratus WEYLAND \& GREIFELD in Frage komme.

Botanische Zugehörigkeit: Lygodium-Spore.

\section{Corrugatisporites sp.}

Taf. 26, Fig. 2a, b.

Trilete Spore. Größter Durchmesser $50 \mu$. Figura kugelig. Äquatorkontur rundlich. Y-Marke nicht erkennbar. Tori sind nicht zu erkennen. Dünnwandiges Exospor vereinigt sehr eng. Warzen sind teils isoliert, teils zu unregelmäßig angeordneten Muri verschmolzen, diese rugulat angeordnet. Warzen $\pm 1.5 \mu$ hoch.

Diese Form ist der japanischen tertiären Spezies Corrugatisporites haradae 
TAKahashi (1961, S. 283-284, Taf. 14, Fig. 1-3, 5-9), ähnlich. Die Warzen der ersteren sind feiner als die der letzteren skulpturiert.

H. D. Pflug (1959) hat zwei morphologisch ähnliche Formen, Corrugatisporites sextarius Pflug (1959, S. 146, Taf. 12, Fig. 1, 5, 6) und Corrugatisporites tindarensis Pflug (1959, S. 146-147, Taf. 12, Fig. 2), aus dem eozänen Tröllatunga-Bild von Husavik-urkleif und Tindar Islands beschrieben. Die vorliegende Spezies ist diesen beiden nordeuropäischen Arten sehr ähnlich. Aber die beiden besitzen dickwandiges Exopsor.

Botanische Zugehörigkeit: Osmundaceae.

Formgattung: Rugulatisporites Thomson \& PfLug

1953. Rugulatisporites Thomson \& PfLUg, S. 56.

Rugulatisporites salebrosus $\mathrm{n} . \mathrm{sp}$.

Taf. 26, Fig. 3-5; Taf. 40, Fig. 11a, b.

D i a g n o s e : Trilete Sporen. Ca. 43-62 $\mu$ groß. Figura kugelig. Äquatorkontur rundlich. Y-Leisten schmal, verhältnismäßig kurz, oft nicht erkennbar. Tori sind nicht zu erkennen. Exospor bis $2 \mu$ dick, grob und dicht rugulat. Muri bis $1.5 \mu$ hoch. Sekundäre Deformationen häufig.

H o l o ty p u s : $62 \mu$ groß; Taf. 26, Fig. 4a, b; Präparat GK-V 3133.

L ocus typicus: Hatsune-Sawa, Ooyubari, Yubari-Kohlenfeld, Hokkaido.

Stratigraphisches V erhalten: Diese Spezies wurde bisher nur in den oberkretazeischen Unter-Hakobuchi-Schichten von Hatsune-Sawa, Yubari-Kohlenfeld gefunden.

Vergle i c h: Die salebrosus-Form ist der mitteleuropäischen mittel- und jungtertiären Spezies, Rugulatisporites quintus Thomson \& PFLUG (P. W. ThomSoN \& H. D. Pflug, 1953, S. 56, Taf. 2, Fig. 44-47) morphologisch ähnlich. Die mitteleuropäische Spezies ist feiner als bei der salebrosus-Form skulpturiert.

Die Figur 6 der Tafel 12 von Corrugatisporites sextarius PFLUG (1959) ist der vorliegenden salebrosus-Form sehr ähnlich.

A. C. Ibrahim (1933) bildet morphologisch ähnliche Formen, Verrucosisporites microverrucosus IBRAHIM (S. 25, Taf. 7, Fig. 10), Verrucosisporites papillosus IBRAHIM (S. 25, Taf. 5, Fig. 44) und Verrucosisporites verrucosus Ibrahim (S. 25, Taf. 2, Fig. 17; R. Potonié \& G. Kremp, 1955, S. 69, Taf. 13, Fig. 196-199), aus den oberkarbonischen Schichten im Ruhr-Gebiet (Deutschland) ab.

G. E. Rouse (1957) hat Verrucosisporites verrucosus IBRAhim (S. 356, Taf. 1, Fig. 25, 26) und Sporites solirlus R. Pot. (in Rouse, Taf. 1, Fig. 27-28; in P. W. Thomson \& H. D. Pflug, Corrugatisporites solidus) aus den oberkretazeischen Comox-Schichten (Campan) von der Vancouver Insel (W-Kanada) abgebildet.

I. C. Cookson (1947) hat ähnliche Form Trilites grandis Cookson (S. 137, 
Taf. 16, Fig. 66-67) aus den tertiären Braunkohlen und Sandsteinen von Waterfall Gorge, Kerguelen Insel, mitgeteilt.

Die salebrosus-Form ist den Figuren $2 \mathrm{a}$ und $2 \mathrm{~b}$ der Tafel 26 in dieser Arbeit, Corrugatisporites sp., ähnlich, aber die erstere kann nach Skulpturelementen von der letzteren unterschieden werden.

Botanische Zugehörigkeit: Osmundaceae.

Rugulatisporites parvirugulatus $\mathrm{n}$. sp.

Taf. 26, Fig. 6-8.

D i a g n o s e : Trilete Sporen. Ca. $32-36 \mu$ groß. Äquatorkontur dreieckig mit konvexen Seiten und abgerundeten Ecken. Y-Leisten erreichen fast den Äquator oder nicht. Exospor dünn, unter $1 \mu$ dick, fein rugulat. Spore reißt leicht in der Y-Marke auf.

H ol oty pus: $33.5 \mu$ groß; Taf. 26, Fig. 6; Präparat GK-V 3143.

Locus typicus: Hatsune-Sawa, Ooyubari, Yubari-Kohlenfeld, Hokkaido.

Stratigraphisches Verhalten: Diese Spezies wurde bisher nur in den oberkretazeischen Unter-Hakobuchi-Schichten (Campan) von HatsuneSawa, Yubari-Kohlenfeld gefunden.

Vergle ich: Die parvirugulatus-Form ist der anderen Art, Punctatisporites punctulatus (in dieser Arbeit, Taf. 25, Fig. 7-15), ähnlich, aber die letztere ist punctat skulpturiert und die Y-Leisten der letzteren sind stärker als die der ersteren.

Die mitteleuropäische tertiäre Spezies, Rugulatisporites quintus THомsoN \& Pflug (P. W. Thomson \& H. D. Pflug, 1953, S. 56, Taf. 2, Fig. 44-47), ist größer als die parvirugulatus-Form und grob rugulat skulpturiert.

G. LESCHIK (1955) hat eine mesozoische Spezies, Rugulatisporites artimuralis LeschIK (R. KRÄUSEL \& G. LeSCHIK, 1955, S. 14, Taf. 1, Fig. 13), aus dem oberen triassischen (Mittelkeuper) sandigen Ton von Neuwelt bei Basel der Schweiz beschrieben. Die Y-Leisten dieser Spezies erreichen den Äquator, aber die der parvirugulatus-Form nicht.

Botanische Zugehörigkeit: Osmundaceae.

\section{Formgattung: Microreticulatisporites (KNOx) R. PoToNIÉ \& KREMP}

1950. Microreticulatisporites KNox, S. 320.

1955. Microreticulatisporites (KNox) PotoniÉ \& KREMP, S. 96.

\section{? Microreticulatisporites sp.}

Taf. 26, Fig. 10.

Die vorliegende Form ist trilete Spore (?) und $22.3 \mu$ groß. Äquatorkontur dreieckig mit konvexen Seiten und abgerundeten Ecken. Y-Leisten (?) schmal, den Äquator erreichend. Exine extrareticulat mit kleinen Lumina, deren Durchmesser unter $0.5 \mu$. 
Sie wurde nur im Kohlenflöz der unteren Hakobuchi-Schichten (Campan) von Hatsune-Sawa, Ooyubari, Yubari-Kohlenfeld, Hokkaido gefunden.

G. E. RousE (1957) hat Microreticulatisporites nobilis (WICHTER) KNOX aus den Comox-Schichten (Campan) von der Vancouver Insel, Westkanada mitgeteilt. Diese Spezies ist der des Karbons im Ruhrgebiet (Deutschland). Die betreffende Form ist kleiner als die nobilis-Form.

Botanische Zugehörigkeit: fraglich.

\section{Microreticulatisporites sp.}

Taf. 26, Fig. 11.

Die rundliche Form ist trilete Spore und $40 \mu$ groß. Äquatorkontur kreisförmig. Y-Leisten schwach, schmal, verhältnismäßig kurz, den Äquator nicht erreichend. Exine extrareticulat mit kleinen Lumen der verschiedenen Formen, deren Durchmesser bis $3 \mu$.

Sie wurde nur im Kohlenflöz der unteren Hakobuchi-Schichtengruppe (Campan) von Hatsune-Sawa, Ooyubari, Yubari-Kohlenfeld, Hokkaido gefunden.

Einige paläozoische Formen (Microreticulatisporites microtuberosus, Mieroreticulatisporites reticuloides, Microreticulatisporites sifati, Microreticulatisporites verus usw.) sind der vorliegenden Form morphologisch ähnlich, aber die ersteren sind im allgemeinen viel größer als die letztere.

Botanische Zugehörigkeit: fraglich.

Formgattung: Lycopodiumsporites (ThIERGART) DELCoURT \& SPRUMONT

1956. Lycopodiumsporites (ThIergarT) Delcourt \& SPRUmont in Potonie, S. 4546.

\section{Lycopodiumsporites yubariensis n. sp. \\ Taf. 40, Fig. 6, 7a, b.}

D i a $\mathrm{g}$ n o s e : Trilete Sporen. Ca. $30-35.5 \mu$ groß. Äquatorkontur subtriangulär. Y-Leisten geradlinig verlaufend, den Äquator erreichend. Exine reticulat. Netzlumen ca. 4 bis $10 \mu$ breit. Muri 1 bis $2.5 \mu$ hoch.

H o loty pus : Ca. $35.5 \mu$ groß; Taf. 40, Fig. 7a, b; Präparat GK-V 3212.

Locus typicus: Bei der Talspere von Ooyubari, Yubari-Kohlenfeld, Hokkaido.

Stratigraphisches Verhalten: Diese Spezies wurde bisher nur in den Sandsteinen der oberen Hakobuchi-Schichtengruppe (Maastricht) bei der Talspere von Ooyubari, Yubari-Kohlenfeld, Hokkaido gefunden.

Vergle i ch: R. Potonié (1934) hat Sporites agathoecus (S. 43, Tab. 1, Fig. 25) aus den eozänen Braunkohlen von Geiseltal (Deutschland) beschrieben. Später hat F. THIERGART (1938) diese Spezies als Lycopodiumsporites agathoecus beschrieben, aber nach R. PотоNiÉ (1956) wurde der Genotyp von DelcourT \& SPRUMONT (1955) gewählt.

P. W. Thomson und H. D. Pflug (1953) hat Reticulatisporites potoniei 
Thomson \& Pflug (S. 55, Taf. 2, Fig. 34; H. Weyland \& G. Greifeld, 1953, S. 41, Taf. 10, Fig. 38-39, 41, 43, 47; cf. Taf. 11, Fig. 61) aus den tertiären und untersenonischen Schichten von Deutschland beschrieben und später hat $R$. PotoniÉ (1956, S. 46) diese Spezies mit der Gattung Lycopodiumsporites neu kombiniert. Die Y-Leisten von Lycopodiumsporites potoniéi (THomson \& PFLUG) Potonié verlaufen strichförmig schmal und erreichen nicht den Äquator.

Lycopodiumsporites triarcuatus Delcourt \& SPRUmont wurde von A. DeLCOURT und G. Sprumont (1955, S. 32, Taf. 3, Fig. 1) aus den unterkretazeischen Schichten (Wealden) von Hainaut, Belgien beschrieben. Diese Spezies besitzt die Äquatorkontur des Dreiecks mit leicht konkaven Seiten.

I. C. Cookson (1953) hat Lycopodium austroclavatidites Cookson (S. 469, Taf. 2, Fig. 35) aus den prätertiären Tonen, Südaustralien beschrieben. Diese Spezies ist größer als die yubariensis-Form. I. C. Cookson und M. E. DetTMANN (1958) haben Lycopodiumsporites circolumenus Cookson \& DetTMaNN (S. 105, Taf. 15, Fig. 10-11) aus den unterkretazeischen Schichten (Neokom-Apt) von Victoria, Australien beschrieben. Diese Form ist größer als die yubariensisForm und die Muri der ersteren sind 1-2 $\mu$ breit und bilden fast kreisförmig oder polygonale Lumina (4-7 $\mu$ im Durchmesser).

Botanische Zugehörigkeit: cf. Lycopodium.

Formgattung: Apiculatisporis R. PoTonié \& KREMP

1956. Apiculatisporis R. Potoní \& KREMP, S. 94.

R. Potonié und Kremp (1956, Teil III, S. 94) haben die neue Gattung Apiculatisporis eingeführt, um die Forderung des Internationalen Codes zu genügen, da die Gattung Apiculatisporites, die von IBRAHIm (1933) geschaffen wurde, in der Gattung Tuberculatisporites verschwindet.

\section{Apiculatisporis inouei n. sp.}

Taf. 27, Fig. 2-9.

Diagnose : Trilete Sporen. Größter Durchmesser 36-62 $\mu$. Figura kugelig bis dick linsenförmig. Äquatorkontur rundlich bis etwas elliptisch. Y-Leisten schmal, verhältnismäßig lang, den Äquator fast oder nicht erreichend, oft nicht erkennen. Dünnwandiges Exospor mit kleinen Zierelementen, die verhältnismäßig licht angeordnet. Coni sind kegelförmig, aber gelegentlich auch terminal abgestumpft; diese bis $1.5 \mu$ hoch. Warzen wenig. Spore reißt oft leicht in der Y-Marke auf. Häufig stark verfaltet.

H o lotypus : Ca. $54 \mu$ groß; Taf. 27, Fig. 6; Präparat GK-V 3152.

Locus typicus: Hatsune-Sawa, Ooyubari, Yubari-Kohlenfeld, Hokkaido.

Stratigraphisches Verhalten: Man kann diese Spezies in der oberen und unteren Hakobuchi-Schichtengruppe (Campan und Maastricht) von Ooyubari, Yubari-Kohlenfeld finden. 
Vergle i ch : I. C. Cookson und M. E. Detrmann (1958) haben Apiculatisporis asymmetricus Cookson \& DetTmann (S. 100, Taf. 14, Fig. 11-12) aus den albischen Schichten von Victoria, Australien und Apiculatisporis wonthaggiensis Cookson \& DetTmanN (S. 100, Taf. 14, Fig. 7-10) aus den aptischen Schichten von Victoria, Australien beschrieben. Die wonthaggiensis-Form ist der inouei-Form mehr ähnlich als die asymmetricus-Form. Die Exine der wonthaggiensis-Form ist viel dicker als die der inouei-Form.

G. LESCHIK (1955) hat Apiculatisporites parvispinosus LESCHIK (in KRÄUSEL \& Leschik, S. 17, Taf. 2, Fig. 1-4), Apiculatisporites spiniger LESCHIK, (S. 18, Taf. 2, Fig. 6-7) und Apiculatisporites globosus LESCHIK (in KRÄUSEL \& Leschik, S. 18, Taf. 2, Fig. 8) aus den oberen triassischen feinsandigen Tonen (Mittelkeuper) von Neuwelt bei Basel der Schweiz beschrieben. Die parvispinosus-Form ist klein und besitzt sehr kleine Stacheln. Das Exospor der spiniger-Form ist zweischichtig ( $2 \mu$ dick) und auf ihm sitzen Dornen von $2.5 \mu$ Höhe. Diese spiniger-Art mag zu der Gattung Tuberculatisporites gehören könn€n. Das Exospor der globosus-Form ist zweischichtig (3.5 $\mu$ dick) und ihre Y-Leisten begleiten die $3 \mu$ breiten bandartigen Wülsten zu beiden Seiten.

G. E. Rouse (1957) hat Osmundasporites primarius WolfF (S. 362, Taf. 3, Fig. 57-58) und Osmundasporites elongatus Rouse (S. 362, Taf. 3, Fig. 59-60) aus den oberkretazeischen Oldman und Brauzeau Schichten von Südalberta, Westkanada abgebildet und beschrieben. Die Gattung Osmundasporites ist nomenklatorisch nicht gültig (vgl. R. РотоNiÉ, 1956, S. 34). Osmundasporites primarius (Sporites primarius WoLfF, 1934, S. 66, Taf. 5, Fig. 8) gehört zu Baculatisporites THOMson \& PFLUG, 1953. Der Verfasser kann hier nicht bestimmen, Osmundasporites elongatus Rouse zu Baculatisporites THOMSON \& Pflug (1953) oder zu Osmundacidites CouPER (1953) zu gehören.

R. PotoniÉ und G. KRemP (1955) haben einige ähnliche paläozoische Spezies von Apiculatisporites (Apiculatisporis, 1956) abgetildet und beschrieben: Apiculatisporis (al. Apiculatisporites) aculeatus (IвRAнim) Рот. \& КremP, Apiculatisporis (al. Apiculatisporites) cf. setulosus (Kosanke) Рот. \& KREMP, Apiculatisporis (al. Apiculatisporites) triangularis (Kosanke) Рот. \& KRemp.

Botanische Zugehörigkeit: Osmundaceae kommen in Frage.

\section{Apiculatisporis micracanthus $\mathrm{n}$. $\mathrm{sp}$.} Taf. 28, Fig. 4a-b, 5.

D i a $\mathrm{g}$ n o s e : Trilete Sporen. Ca. 36-42 $\mu$ groß. Äquatorkontur dreieckig mit konvexen Seiten und mehr oder weniger abgerundeten Ecken. Y-Leisten schmal oder wulstig (1.4 $\mu$ breit), den Äquator ganz oder fast erreichend. Exospor bis $1.2 \mu$ dick, mit kleinen Coni oder selten Stäbchen. Coni (oder selten Stäbchen) bis $2 \mu$ hoch in verhältnismäßig dichter Anordnung. Spore reißt leicht in der Y-Marke auf.

H o l ot y p u s : Ca. $36 \mu$ groß; Taf. 28, Fig. 4a, b; Präparat GK-V 3155.

Locus typicus: Hatsune-Sawa, Ooyubari, Yubari-Kohlenfeld, Hok- 
kaido.

Stratigraphisches Verhalten: Diese Spezies tritt bisher nur im Kohlenflöz der oberkretazeischen Unter-Hakobuchi-Schichten (Campan) von Hatsune-Sawa, Yubari-Kohlenfeld auf.

Vergle i ch: R. Potonié und G. KREmP (1955) haben die paläozoische dreieckige Spezies, Apiculatisporis (al. Apiculatisporites) cf. setulosus (KoSANKe) PoT. \& KRemP (S. 79-80, Taf. 14, Fig. 246-248), aus dem Karbon im Ruhrgebiet (Deutschland) mitgeteilt. Die Äquatorkontur dieser Spezies ist der micracanthus-Form sehr ähnlich, aber die Größe der ersteren ist zweimal größer als die der letzteren.

Botanische Zugehörigkeit: fraglich.

Formgattung: Baculatisporites THomson \& PfLUG

1953. Baculatisporites Thomson \& Pflug, S. 56.

Baculatisporites papillosus n. sp.

Taf. 27, Fig. 10-15; Taf. 28, Fig. 1-3.

Diagnose: Trilete Sporen Größter Durchmesser 41-70 $\mu$. Figura ursprünglich kugelig bis dick linsenförmig. Äquatorkontur rundlich bis schwach dreieckig. Y-Leisten schmal, verhältnismäßig lang, den Äquator nicht erreichend, oft nicht erkennbar. Exospor 1 bis $1.2 \mu$ dick, mit baculaten, verrucaten oder sehr selten echinaten Zierelementen in verhältnismäßig dichter Anordnung. Stäbchen bis $4.2 \mu$ hoch (häufigst unter $2 \mu$ hoch). Spore reißt oft leicht in der Y-Marke auf. Häufig stark verfaltet.

H o loty p u s : $55.3 \mu$ groß; Taf. 27, Fig. 12; Präparat GK-V 3132.

Locus typicus: Hatsune-Sawa, Ooyubari, Yubari-Kohlenfeld, Hokkaido.

Stratigraphisches Verhalten: Diese Spezies kann man bisher nur im Kohlenflöz der unteren Hakobuchi-Schichtengruppe (Campan) von Hatsune-Sawa, Yubari-Kohlenfeld finden.

Vergleich: Die vorliegende Form ist der validus-Form (in dieser Arbeit, Taf. 28, Fig. 6-11) nähest verwandtschaftlich. Das Exospor der ersteren ist dicker als das der letzteren; die erstere kann hauptsächlich nach Dicke der Exine von der letzteren unterschieden werden.

Die papillosus-Form ist weiter der mitteleuropäischen tertiären Spezies, Baculatisporites primarius (WoLFF) THOMSON \& PFLUG (Sporites primarius WolfF, 1934, S. 66, Taf. 5, Fig. 8; P. W. Thomson \& H. D. Pflug, 1953, S. 56, Taf. 2, Fig. 49-53), ähnlich, aber die erstere kann nach Höhe und Anordnung der Stäbchen von der letzteren unterschieden werden.

I. C. Cookson (1953) hat morphologisch ähnliche Spezies, Triletes comaumensis Cookson (S. 470, Taf. 2, Fig. 27-28), aus den prätertiären Tonen von Comaum, Südaustralien beschrieben. Diese Art gehört zu der Gattung Baculatisporites. Die comaumensis-Form kann nach Dicke der Exine und Höhe der 
Skulpturelemente von der papillosus-Form unterschieden werden.

S. MANUM (1962) hat morphologisch sehr ähnliche Form, Baculatisporites cf. gemmatus KrutzSCH (in S. MANUM, 1962, S. 22, Taf. 1, Fig. 8-9), aus den tertiären Schichten von Spitzbergen abgebildet und beschrieben. Die gemmatusForm ist etwas größer als die papillosus-Form und die Exine der ersteren ist etwas dünner als die der letzteren.

Botanische Zugehörigkeit: Osmundaceae.

Baculatisporites validus $\mathrm{n}$. sp.

Taf. 28, Fig. 6-11.

D i a g n o s e : Trilete Sporen. Größter Durchmesser ca. 30.5-65 $\mu$. Figura ursprünglich kugelig bis dick-linsenförmig. Äquatorkontur rundlich bis schwach dreieckig. Y-Leisten schmal (selten wulstig, $1.4 \mu$ breit), lang, den Äquator fast oder nicht erreichend, oft nicht erkennbar. Exine sehr dünn, mit baculaten, verrucaten oder selten kegelförmigen Zierelementen in verhältnismäßig dichter Anordnung. Stäbchen bis $2.5 \mu$ hoch und an der Basis bis $2.7 \mu$ breit. Spore reißt leicht in der Y-Marke auf. Oft verfaltet.

H o lotypus : $54.4 \mu$ groß; Taf. 28, Fig. 9; Präparat GK-V 3143.

Locus typicus: Hatsune-Sawa, Ooyubari, Yubari-Kohlenfeld, Hokkaido.

Stratigraphisches Verhalten: Man kann diese Spezies in der oberen (Maastricht) und unteren (Campan) Hakobuchi-Schichtengruppe von Ooyubari, Yubari-Kohlenfeld finden.

Vergle i ch: Diese Form ist der Art, Baculatisporites papillosus TAKAHASHI (in dieser Arbeit, Taf. 27, Fig. 10-15; Taf. 28, Fig. 1-3), nähest ähnlich, aber die erstere kann nach Dicke der Exine von der letzteren unterschieden werden.

I. C. Cookson (1953) hat Triletes comaumensis Cookson (S. 470, Taf. 2, Fig. 27-28) beschrieben. Diese australische Form kann nach Größe der Stäbchen von der validus-Form unterschieden werden.

S. MANum (1962) hat die sehr ähnliche Spezies, Baculatisporites cf. gemmatus KRUTZSCH, aus den tertiären Schichten von Spitzbergen mitgeteilt.

Botanische Zugehörigkeit: Osmundaceae.

Formgattung: Neoraistrickia R. PoToNiÉ

1956. Neoraistrickia R. PotonIE, S. 34.

Neoraistrickia sp.

Taf. 40, Fig. 8a, b.

Trilete Spore. Größter Durchmesser $26.7 \mu$. Äquatorkontur dreieckig mit schwach konvexen Seiten und abgerundeten Ecken. Y-Leisten wulstig?, den Äquator erreichend. Exine dünn mit baculaten und selten kegelförmigen Zier- 
elementen in lichter Anordnung. Stäbchen bis $1.5 \mu$ hoch.

Diese Form wurde nur im Feinsandstein der oberen Hakobuchi-Schichtengruppe (Maastricht) bei der Talspere von Ooyubari, Yubari-Kohlenfeld, Hokkaido gefunden.

I. C. Cookson (1953) hat Neoraistrickia (al. Trilites) truncatus (Cookson) R. PotoniÉ aus den prätertiären Schichten von Südaustralien beschrieben.

R. A. COUPER (1953) hat Neoraistrickia (al. Raistrickia) neozealandica (CoUPER) R. PotoniÉ aus den jurassischen Ohika-Schichten von Buller Gorge, Neuseeland beschrieben.

Botanische Zugehörigkeit: fraglich.

Formgattung: Tuberculatisporites (IBRAhIm) R. PotoniÉ \& KREMP

1933. Tuberculatisporites IвRAHIM, S. 22.

1956. Tuberculatisporites (IBRAHIM) ex Potonit \& KREMP, S. 88.

Tuberculatisporites sp.

Taf. 40, Fig. 9a, b.

Trilete Spore (?). Ca. $32 \mu$ groß. Figura ursprünglich kugelig bis dick linsenförmig. Äquatorkontur ursprünglich rundlich. Y-Leisten sind nicht zu erkennen. Exospor sehr dünn, einschichtig. Echinat. Stacheln bis $1.6 \mu$ lang in lichter Anordnung.

Diese Form wurde bisher nur im Feinsandstein der oberen HakobuchiSchichtengruppe (Maastricht) bei der Talspere von Ooyubari, Yubari-Kohlenfeld, Hokkaido gefunden.

Botanische Zugehörigkeit: fraglich.

\section{? Tuberculatisporites $\mathrm{sp}$. \\ Taf. 41, Fig. 4.}

Trilete Spore (?). Ca. $16 \mu$ groß. Figura ursprünglich kugelig. Äquatorkontur rundlich. Y-Marke ist nicht zu erkennen. Exospor dünn, glatt. Echinat. Stacheln fein, unter $1 \mu$ lang.

Diese Spezies wurde bisher nur im Feinsandstein der oberen HakobuchiSchichtengruppe (Maastricht) bei der Talspere von Ooyubari, Yubari-Kohlenfeld, Hokkaido gefunden.

Botanische Zugehörigkeit: fraglich.

Formgattung: Triplanosporites PFLUG

1953. Triplanosporites Pflug in Thomson \& Pflug, S. 58.

Triplanosporites sinuatus $\mathrm{n}$. $\mathrm{sp}$.

Taf. 28, Fig. 12-14.

D i a g n o s e : Ca. 31-33 (Länge der Polachse). Äquatorkontur dreiec- 
kig mit extrem konkaven Seiten. Y-Leisten schmal, verhältnismäßig kurz, den Äquator nicht erreichend. Exospor dünn (unter $1 \mu$ ), chagrenat bis fein rugulat oder schwach glatt.

H o l o t y p u s : $31.2 \mu$ groß; Taf. 28, Fig. 14; Präparat GK-V 3161.

Locus typicus: Hatsune-Sawa, Ooyubari, Yubari-Kohlenfeld, Hokkaido.

Stratigraphisches Verhalten: Man konnte diese Form nur im Kohlenflöz der unteren Hakobuchi-Schichtengruppe (Campan) von HatsuneSawa, Yubari-Kohlenfeld finden.

Vergleich: Die betreffende Spezies ist der mitteleuropäischen danischen (?) bis paläozänischen Spezies, Triplanosporites sinuosus Pflug (P. W. Thomson \& H. D. Pflug, 1953, S. 58, Taf. 3, Fig. 5-16), ähnlich. Die europäische Spezies ist im allgemeinen größer und ihre Exine ist glatt.

Die oligozäne Form, Triplanosporites sp. (Kasuya-Form) (K. TaKaHASHI, 1961, S. 288, Taf. 14, Fig. 12), die von dem Verfasser aus dem Itsue-Flöz der Shinbaru-Schichten im Kasuya-Kohlenfeld, Kyushu mitgeteilt wurde, besitzt morphologisch ähnliche Form. Ihre Exospor ist glatt und ca. $1 \mu$ dick. Die Y-Marke ist deutlich.

S. SAto (1961) hat Deltoidospora enbetsuensis SATo (S. 88, Taf. 2, Fig. 1-5) aus den oberkretazeischen Osoushinai-Schichten von Nordhokkaido beschrieben. Besonders sind die Figuren 2 und 4 davon der sinuatus-Form ähnlich, aber das Exospor der ersteren ist punctat oder fein granulat.

Botanische Zugehörigkeit: fraglich.

\section{Zonales}

Formgattung: Biretisporites DeLCoURT \& SPRUmonT

1955. Biretisporites Delcourt \& SpRumont, S. 40-41.

1956. Biretisporites Delcourt \& SPRUMont in PotoniÉ, S. 54.

Biretisporites ? minus n. sp.

Taf. 26, Fig. 9; Taf. 40, Fig. 4a, b.

D i a g nos e : Trilete Sporen. Ca. $23-27 \mu$ groß. Äquatorkontur mit konvexen Seiten und mehr oder weniger gespitzten Ecken. Y-Strahlen kräftig (wulstig?), 1.4 bis $1.6 \mu$ breit, mehr oder weniger bogig verlaufend, den Äquator erreichend. Exospor sehr dünn, punctat bis schwach rugulat.

H o l oty p u s : Ca. $27 \mu$ groß; Taf. 26, Fig. 9 ; Präparat GK-V 3181.

Locus typicus: Hatsune-Sawa, Ooyubari, Yubari-Kohlenfeld, Hokkaido.

Stratigraphisches Verhalten: Diese kleine Form wurde in der oberen und unteren Hakobuchi-Schichtengruppe (Maastricht und Campan) von Ooyubari, Yubari-Kohlenfeld gefunden.

Vergleich: A. Delcourt und G. Sprumont (1955) haben die neue Gattung Biretisporites geschaffen. Biretisporites potoniaei DeLCoURT \& SPRU- 
MONT vertritt die Gattung Biretisporites.

Die japanische kleine Form gehört noch etwas zweifelhaft zu Biretisporites. Botanische Zugehörigkeit: fraglich.

\section{Monoletes}

Formgattung: Laevigatosporites (IBRAhIm) R. Potonié \& KREMP

1933. Laevigatosporites IBRAhIM, S. 39.

1937. Polypodiumsporites RAATZ, S. 10.

1956. Laevigatosporites (IBRAhim) ex Potoní́ \& KremP, S. 137.

1956. Polypodiaceaesporites Thiergart ex R. PotoniE, S. 76.

R. Рото̃IÉ (1956) hat Laevigatosporites für die paläozoischen Formen und Polypodiaceaesporites für die jüngeren Formen benutzt. Aber er gibt keine morphologische Eigentümlichkeit, die Laevigatosporites von Polypodiaceaesporites unterscheiden kann. Deshalb hat die Gattung Laevigatosporites eine Priorität.

Laevigatosporites prominens $\mathrm{n}$. sp.

Taf. 29, Fig. 1-7; Taf. 40, Fig. 12, 18.

D i a g n o s e : Monolete Sporen. 37.5-50 $\mu$ groß. Figura ellipsoidisch bis bohnenförmig. Kontur an der Dehiszenz schwach konkav. Dehiszenz verhältnismäßig kurz, etwas wulstig, den Äquator nicht erreichend. Exospor 1 bis $2 \mu$ dick, chagrenat.

H o lo ty pus : $43.3 \mu$ groß; Taf. 29, Fig. 5 ; Präparat GK-V 3141.

Locus typicus: Hatsune-Sawa Ooyubari, Yubari-Kohlenfeld, Hokkaido.

Stratigraphisches Verhalten: Diese Form wurde bisher nur in der oberen (Maastricht) und unteren (Campan) Hakobuchi-Schichtengruppe von Ooyubari, Yubari-Kohlenfeld gefunden.

Vergleich: In Japan wurde schon fünf neue Spezies von Laevigatosporites aus den alttertiären und miozänen Schichten von Westjapan von dem Verfasser beschrieben: Laevigatosporites dehiscens TAKAHASH (1961, S. 290, Taf. 16, Fig. 4-8), Laevigatosporites mikawaensis TAKAHASHI (1961, S. 289-290, Taf. 15, Fig. 16), Laevigatosporites gigantiformis TAKAHASHI (1961, S. 189, Taf. 16, Fig. 3), Laevigatosporites ovoideus TAKAhashi (1961, S. 288-289, Taf. 16, Fig. 9-14) und Laevigatosporites eogigantiformis TAKAHASHI (1962, S. 11, Taf. 1, Fig. 19-21; Taf. 2, Fig. 1-4). Die vorliegende Spezies ist Laevigatosporites mikawaensis TAKAHASHI und Laevigatosporites dehiscens TAKAHASHI morphologisch ähnlich, aber die erstere kann nach Struktur der Exine von der mikawaensis-Form und nach Dicke der Exine von der dehiseens-Form unterschieden werden. S. SATo (1961) hat den amerikanische Spezies Laevigatosporites gracilis WILSON \& WEBSTER und Laevigatosporites ovatus WILSON \& WEBSTER aus den oberkretazeischen Osoushinai-Schichten von Nordhokkaido 
abgebildet.

In Kanada hat G. E. Rouse Laevigatosporites ovatus WILsON \& WEBSTER (1957, S. 355, Taf. 1, Fig. 3) aus den campanischen Comox-Schichten, Laevigatosporites albertensis Rouse (1957, S. 363-364, Taf. 2, Fig. 17, 18; 1962, S. 198, Taf. 1, Fig. 25-26), Laevigatosporites ovatus WILson \& WEBSTER (1957, S. 364, Taf. 2, Fig. 19, 20; 1962, S. 198, Taf. 2, Fig. 1-2) und Laevigatosporites discordatus Thomson \& Pflug (1957, S. 364, Taf. 2, Fig. 21, 22 ; 1962, S. 198, Taf. 2, Fig. 3) aus den campanischen Oldman-Schichten und aus den eozänen BurrardSchichten abgebildet und beschrieben.

In USA haben L. R. WILSON und R. M. WeBSTER (1946) Laevigatosporites gracilis WILsON \& WeBsTER (S. 273, Fig. 4) und Laevigatosporites ovatus WiLsoN \& WEBSTER (S. 273, Fig. 5) aus den paläozänen Fort Union Schichten (Montana) beschrieben. Die prominens-Form ist größer als die gracilis- und ovatus-Form.

I. C. Cookson (1947) hat Monolites major Cookson (S. 135, Taf. 15, Fig. 56) und Monolites minor Cookson (S. 135, Taf. 15, Fig. 57) aus den tertiären Braunkohlen und Sandsteinen von der Kerguelen Insel beschrieben. Monolites ist Synonym von Laevigatosporites nach dem Urteil der Beschreibungen und Photos. Die Exine der minor-Form ist glatt und zart.

Die betreffende Spezies ist auch der mitteleuropäischen Art, Laevigatosporites haardti (R. PoT. \& Ven.) Thomson \& PFLUg (Sporites haardti R. Рот. \& Ven., 1934, S. 13, Taf. 1, Fig. 13; P. W. Thomson \& H. D. Pflug, 1953, S. 59, Taf. 3, Fig. 27-38) ähnlich.

Botanische Zugehörigkeit: Polypodiaceae.

\section{Laevigatosporites probatus n. sp. \\ Taf. 29, Fig. 8.}

D i a $\mathrm{g}$ n o s e : Monolete Sporen. Ca. 41-52.5 $\mu$ groß. Figura breit-ellipsoidisch bis breit-bohnenförmig. Kontur an der Dehiszenz konvex oder konkav. Dehiszens nicht wulstig, verhältnismäßig kurz, beiderseits den Äquator nicht erreichend. Exospor sehr dünn, schwach chagrenat.

H o loty p u s : Ca. $43 \mu$ groß; Taf. 29, Fig. 8; Präparat GK-V 3131.

Locus typicus: Hatsune-Sawa, Ooyubari, Yubari-Kohlenfeld, Hokkaido.

Stratigraphisches Verhalten: Man kann diese Spezies in der oberen (Maastricht) und unteren (Campan) Hakobuchi-Schichtengruppe von Ooyubari, Yubari-Kohlenfeld finden.

Vergleich : Diese Spezies ist der amerikanischen paläozänen Spezies, Laevigatosporites ovatus WILsON \& WEBSTER (1946, S. 273, Fig. 5), aus den Fort Union Schichten ähnlich. Die erstere ist größer als die letztere und die Exine der ersteren ist dünner als die der letzteren.

I. C. Cookson (1947) hat morphologisch ähnliche Spezies, Monolites major Cookson (S. 135, Taf. 15, Fig. 56), aus den tertiären Braunkohlen und Sandsteinen von der Kerguelen Insel beschrieben. Die probatus-Form ist viel kleiner 
als die major-Form.

Die europäische tertiäre Spezies, Laevigatosporites discordatus PFLUG ( $\mathrm{P}$. W. Thomson \& H. D. Pflug, 1953, S. 59, Taf. 3, Fig. 39-44), ist viel größer als die vorliegende Spezies.

Botanische Zugehörigkeit: Polypodiaceae.

Laevigatosporites dehiscens TAKAHASHI

Taf. 29, Fig. 9-13; Taf. 40, Fig. 13-15.

1961. Laevigatosporites dehiscens, K. TaKahashi, S. 290, Taf. 16, Fig. 4-8.

Diese Spezies wurde vom Verfasser (1961) erstmals beschrieben. Dabei hat er viele diese Körner aus den paläogenen und miozänen Schichten von Westjapan beobachtet. Er hat auch dieselbe Spezies aus den obern und unteren Schichten von Ooyubari im Yubari-Kohlenfeld (Hokkaido) beobachtet.

Die Exine der dehiscens-Form ist dünner (unter $1 \mu$ dick) als die der prominens-Form.

Botanische Zugehörigkeit: Polypodiaceae.

Laevigatosporites senonicus n. sp.

Taf. 29, Fig. 14-19; Taf. 40, Fig. 16, 17.

D i a g n o s e : Monolete Sporen. Ca. 24-42 $\mu$ lang und ca. 18-31.3 $\mu$ breit. Breitenlängenindex 0.7 bis 0.8 . Figura breit-bohnenförmig (nierenförmig) bis breit-ellipsoidisch. Kontur an der Dehiszenz konkav bis schwach konkav oder schwach konvex. Dehiszenz verhältnismäßig kurz, etwas wulstig, beiderseits den Äquator nicht erreichend. Exospor bis $1.5 \mu$ dick, schwach chagrenat.

H o l o ty pu s : Ca. $33 \mu$ groß; Taf. 29, Fig. 14; Präparat GK-V 3141.

Locus typicus: Hatsune-Sawa, Ooyubari, Yubari-Kohlenfeld, Hokkaido.

Stratigraphisches Verhalten: Diese Spezies wurde bisher in der oberen (Maastricht) und unteren (Campan) Hakobuchi-Schichtengruppe von Ooyubari, Yubari-Kohlenfeld gefunden.

Vergleich : Morphologisch sehr ähnliche Spezies Laevigatosporites albertensis Rouse (1957, S. 363-364, Taf. 2, Fig. 17, 18) wurde von G. E. RousE aus den campanischen Oldman-Schichten von Südalberta beschrieben. Diese Form ist schwach punctat skulpturiert. Deshalb kann die senonicus-Form nach Skulptur der Exine von der albertensis-Form unterschieden werden.

Von den Figuren der Tafel 3, die von P. W. Thomson und H. D. Pflug (1953) als Laevigatosporites haardti abgebildet wurden, sind besonders die Figuren 28, 34 und 39 der senonicus-Form sehr ähnlich.

Die betreffende Spezies ist der rezenten Spezies Schizaea bifida Sw. (in N. A. Bolk hovitina, 1961, S. 22-23, Taf. 3, Fig. 8a-c) von Australien, Tasmanien, Neuseeland und Neu-Kaledonien morphologisch ähnlich, aber die Exine der letzteren ist über zweimal dicker als die der ersteren. 
Botanische Zugehörigkeit: Polypodiaceae.

Laevigatosporites ovatus WILSON \& WEBSTER

Taf. 29, Fig. 20.

1946. Laevigatosporites ovatus, L. R. WILSON \& R. M. WeBSTER, S. 273, Fig. 5.

1957. Laevigatosporites ovatus, G. E. Rouse, S. 355, 364; Taf. 1, Fig. 3; Taf. 2, Fig. $19,20$.

Monolete Spore. Ca. $38 \mu$ groß. Figura breit-ellipsoidisch. Dehiszenz verkältnismäßig kurz, beiderseits den Äquator nicht erreichend. Exospor $1.7 \mu$ dick, glatt.

Die betreffende Form gehört sicher zu der amerikanischen paläozänen Form Laevigatosporites ovatus WiLson \& WeBster. G. E. Rouse (1957) hat dieselbe Spezies aus den campanischen Comox- und Oldman-Schichten und den eozänen Burrard-Schichten von Westkanada mitgeteilt. Diese westkanadische Form ist größer als die amerikanische Form. S. SATo (1961) hat die Formen aus den Osoushinai-Schichten als Laevigatosporites ovatus mitgeteilt. Die Form von Sato ist auch größer als die amerikanische originale Form.

Botanische Zugehörigkeit: Polypodiaceae.

Laevigatosporites sp.

Taf. 29, Fig. 21.

Monolete Spore. $70 \mu$ groß. Figura bohnenförmig. Kontur an der Dehiszenz vielleicht geradlinig. Dehiszenz lang, wulstig, bei dieser Figur aufgeplatzt. Exospor $1.7 \mu$ dick, schwach chagrenat.

Diese Form wurde bisher nur im Kohlenflöz der unteren Hakobuchi-Schichten von Hatsune-Sawa, Yubari-Kohlenfeld gefunden. Sie ist Laevigatosporites prominens Takahashi (in dieser Arbeit, Taf. 29, Fig. 1-7; Taf. 40, Fig. 12, 19) und Laevigatosporites eogigantiformis TAKAHASHI (1962, S. 11, Taf. 1, Fig. 19-21; Taf. 2, Fig. 1-4) morphologisch ähnlich. Die erstere ist viel größer als die prominens-Form und die Exine der ersteren ist viel dünner als die der eogigantiformis-Form.

Botanische Zugehörigkeit: Polypodiaceae.

\section{Formgattung: Polypodiisporites R. PotoniÉ}

1933. Polypodii-sporites Pot. in Pot. \& Gell., S. 524 (1962, in Manum, S. 21).

1934. Polypodii-sporites Pot., S. 38.

1956. Polypodiisporites PoT., S. 78.

R. Potonié (1931) hat zuerst Polypodii(?)-sporites favus R. Pot. (S. 556, Fig. 3) mitgeteilt, aber er hat dabei keine Beschreibung und nur einige Bezeichnungen gegeben. Daher ist die Beschreibung von Polypodii-sporites favus Рот. in Potonié \& Gelletich (1933), wie S. MANum (1962, S. 21) behauptet hat, gültig. Später hat R. PotoniÉ (1956, S. 78) die genügende Diagnose für die 
Gattung Polypodiisporites gegeben.

Polypodiisporites, Polypodiidites und Reticuloidosporites besitzen je morphologisch sehr ähnliche Eigentümlichkeiten.

Polypodiisporites repandus n. sp.

Taf. 30, Fig. 1-11; Taf. 31, Fig. 1; Taf. 40, Fig. 22a, b.

D i a g no s e : Monolete Sporen. $40-52 \mu$ groß. Figura ellipsoidisch bis bohnenförmig. Kontur an der Dehiszenz schwach konvex bis schwach konkav. Dehiszenz schmal bis etwas wulstig, verhältnismäßig lang, den Äquator beiderseits nicht erreichend. Exine mit Warzen meistens unter $2 \mu$ hoch, selten bis $3 \mu$ hoch, zwischen ihnen nur ein schmalrinniges negatives Reticulum verbleibt. Netzlumen polygonal, bis $6 \mu$ breit.

H o l o t y p u s : $44.6 \mu$ groß; Taf. 30, Fig. 5a, b; Präparat GK-V 3133.

L ocus typicus: Hatsune-Sawa, Ooyubari, Yubari-Kohlenfeld, Hokkaido.

Stratigraphisches Verhalten: Diese Spezies wurde in der oberen (Maastricht) und unteren (Campan) Hakobuchi-Schichtengruppe von Ooyubari, Yubari-Kohlenfeld (Hokkaido) gefunden.

Vergleich: Die betreffende Spezies ist den mitteleuropäischen tertiären Spezies, Polypodiisporites favus R. Pот. (РотоNIÉ \& Gelletich, 1933, S. 524, Taf. 2, Fig. 14; Verrucatosporites favus in Thomson \& Pflug, 1953, S. 60, Taf. 3, Fig. 52-55) und Reticuloidosporites clathriformis (MÜRRIGER \& Pflug) (P. W. Thomson \& H. D. Pflug, 1953, S. 61, Taf. 4, Fig. 5-8; Polypodiidites clathriformis, R. PотоNIÉ, 1956, S. 79), ähnlich. Besonders ist Polypodiisporites favus R. Рот. sehr ähnlich.

Die repandus-Form ist der westjapanischen eozänen Spezies, Polypodiisporites (al. Reticuloidosporites) nagaii (TAKAHASHI) (K. TAKAHASHI, 1962, S. 13, Taf. 2, Fig. 13-19) ähnlich. Die letztere besitzt etwas flache kuppelförmige Platten.

Botanische Zugehörigkeit: Polypodiaceae.

Polyporliisporites invisus n. sp.

Taf. 31, Fig. 2; Taf. 40, Fig. 20.

D i a g n o s e : Monolete Sporen Ca. 33-47 $\mu$ groß. Figura bohnenförmig. Kontur an der Dehiszenz schwach konkav. Dehiszenz schmal?. Sie ist nicht gut zu erkennen. Etwas flache Warzen in dichter Anordnung unregelmäßig auf der Oberfläche verteilt, bis $3 \mu$ hoch. Grundriß der Warzen verschieden, zum Teil auch verlängert, zwischen ihnen nur ein schmalrinniges negatives Reticulum verbleibt.

H o l o ty pu s : $47 \mu$ groß; Taf. 31, Fig. 2; Präparat GK-V 3152.

Locus typicus: Hatsune-Sawa, Ooyubari, Yubari-Kohlenfeld, Hokkaido.

Stratigraphisches Verhalten: Diese Spezies wurde in der 
oberen und unteren Hakobuchi-Schichtengruppe gefunden.

Ve r g l e i c h : Die invisus-Form ist der westjapanischen eozänen Spezies, Polypodiisporites (al. Reticuloidosporites) nagaii (TAKAнASHI) (1962, S. 13, Taf. 2, Fig. 13-19) ähnlich, aber die flache warzenartige Skulptur der letzteren ist bis $1.5 \mu$ hoch.

Die betreffende Spezies ist der mitteleuropäischen tertiären Spezies, Polypodiisporites favus R. Рот. (РотоNí \& Gelletich, 1933, S. 524, Taf. 2, Fig. 18; R. PotoniÉ, 1934, S. 38, Taf. 1, Fig. 19, 20 ; Verrucatosporites favus in ThomSON \& Pflug, 1953, S. 60, Taf. 3, Fig. 52-55), morphographisch ähnlich. Die Warzen der ersteren ist größer und gröber als die der letzteren.

Botanische Zugehörigkeit: Polypodiaceae.

Formgattung: Verrucatosporites (Thomson \& PfLUg) R. РотоNiÉ

1953. Verrucatosporites Thomson \& Pflug, S. 59.

1956. Verrucatosporites (Thomson \& PfLuG) ex R. PotoniE, S. 79-80.

Verrucatosporites sp. a

Taf. 31, Fig. 3.

Das vorliegende Sporenkorn ist nur halb erhalten.

Monolete Spore. Ca. $60 \mu$ lang? und $22.3 \mu$ breit. Figura bohnenförmig. Kontur an der Dehiszenz geradlinig oder schwach konkav. Dehiszenz schmal (?). Warzen in verhältnismäßig lichter Anordnung unregelmäßig aus der Oberfläche verteilt, bis $1.3 \mu$ hoch. Grundriß der Warzen verschieden, zum Teil verlängert.

Diese Form wurde nur im Kohlenflöz der unteren Hakobuchi Schichtengruppe (Campan) von Hatsune-Sawa, Ooyubari, Yubari-Kohlenfeld, Hokkaido gefunden.

Botanische Zugehörigkeit: Polypodiaceae.

$$
\begin{aligned}
& \text { Verrucatosporites sp. b } \\
& \text { Taf. } 40 \text {, Fig. 21a, b. }
\end{aligned}
$$

Monolete Spore. Ca. $40 \mu$ groß. Figura bohnenförmig. Kontur an der Dehiszenz stark konkav. Dehiszenz schmal. Warzen in sehr lichter Anordnung unregelmäßig auf der Oberfläche verteilt, bis $1.7 \mu$ hoch.

Diese Spore wurde bisher nur im Feinsandstein der oberen HakobuchiSchichtengruppe bei der Talspere von Ooyubari, Yubari-Kohlenfeld (Hokkaido) gefunden.

Botanische Zugehörigkeit: Polypodiaceae?

\section{Formgattung: Undulatosporites LESCHIK}

1955. Undulatosporites LESCHIK in KRÄUSEL \& LESCHIK, S. 28.

1958. Undulatosporites LESCHIK in R. Potoní, S. 32. 


\section{Undulatosporites rugulatus $\mathrm{n}$. sp. \\ Taf. 31, Fig. 4, 5.}

D i g $\mathrm{g}$ o s e : Monolete Sporen. Ca. $40-43 \mu$ groß. Figura breit-ellipsoidisch. Kontur breit-elliptisch oder kreisförmig. Dehiszenz etwas wellig oder geradlinig verlaufend, wulstig $(3.8 \mu$ breit) oder schmal. Exine bedeckt mit grob rugulater Skulptur.

H o l o ty p u s : Ca. $43 \mu$ groß; Taf. 31, Fig. 4; Präparat GK-V 3173.

Locus typicus: Hatsune-Sawa, Ooyubari, Yubari-Kohlenfeld, Hokkaido.

Stratigraphisches Verhalten: Diese Spore wurde bisher nur im Kohlenflöz der unteren Hakobuchi-Schichten (Campan) von HatsuneSawa, Yubari-Kohlenfeld gefunden.

Vergle i ch: G. LeschiK (1955) hat Undulatosporites lucens LESCHIK (in KräUsel \& LeschiK, S. 28, Taf. 3, Fig. 18) aus den obertriassischen Schichten (Mittelkeuper) von Neuewelt bei Basel, Schweiz beschrieben. Dabei hat er die neue Formgattung Undulatosporites vorgeschlagen. Das Exospor der lucens-Form ist $1 \mu$ dick und mit einem Netzwerk von kurzen gebogenen Leistchen überzogen.

Botanische Zugehörigkeit: fraglich.

\section{Formgattung: Punctatosporites IBRAHIM}

1933. Punctatosporites IBRAhIM, S. 40.

1953. Punctatosporites Ibrahim in Thomson \& Pflug, S. 60.

1956. Punctatosporites IBRAHIM in Potoní \& KREMP, S. 141.

1956. Punctatosporites IBRAHIM in R. Potoní, S. 77-78.

\section{Punctatosporites sp.}

Taf. 40, Fig. 19.

Monolete Spore. $36.7 \mu$ groß. Figura bohnenförmig, Kontur an der Dehiszenz schwach konkav. Dehiszenz schmal. Exospor verhältnismäßig dicht punctat (unter $0.5 \mu$ hoch), selten baculat (bis $1.5 \mu$ hoch).

Diese Art wurde bisher nur im Sandstein der oberen Hakobuchi-Schichtengruppe (Maastricht) bei der Talspere von Ooyubari, Yubari-Kohlenfeld (Hokkaido) gefunden.

Botanische Zugehörigkeit: fraglich.

\section{Formgattung: Cicatricososporites THOMsON \& PFLUG}

1953. Cicatricososporites Thomson \& Pflug, S. 61.

1956. Schizaeoisporites R. PotoniÉ, S. 81-82.

R. Potonié (1956, S. 81-82) hat Schizaeoisporites R. PotoniÉ (1951) als gültig behauptet, aber er hat 1951 keine Diagnose von Schizaeoisporites pseudodorogensis R. Рот. gegeben. Deshalb kann man eine Priorität der Gattung 
Cicatricososporites Thomson \& Pflug (1953) behaupten. R. PотоNiÉ hat 1956 eine genügende Beschreibung der Gattung Schizaeoisporites gegeben.

Cicatricososporites ? ellipsoideus n. sp.

Taf. 31, Fig. 6-9.

Di a g n o s e : Monolete Sporen (?) Ca. $32-39 \mu$ groß. Figura ellipsoidisch. Dehiszenz ist nicht $\mathrm{zu}$ erkennen. Exine mit einander parallelen, schmalen, etwas welligen Leisten, d. h. cicatricos. Leisten (Bastionen) 0.5 bis $0.6 \mu$ breit, in der 5.5 bis $8 \mu$ Breite 3 Stücke vorhanden, der Polachse schief verlaufend. Sie konvergieren \pm in Richtung der Schmalseiten des Äquators.

H ol o ty p u s : Ca. $39 \mu$ lang, $17 \mu$ breit; Taf. 31, Fig. 7; Präparat GK-V 3143.

Locus typicus: Hatsune-Sawa, Ooyubari, Yubari-Kohlenfeld, Hokkaido.

Stratigraphisches Verhalten: Diese Spezies wurde bisher nur im Kohlenflöz der unteren Hakobuchi-Schichtengruppe (Campan) von Hatsune-Sawa, Yubari-Kohlenfeld gefunden.

Vergleich: Cicatricososporites pseudodorogensis (R. Рот.) Thоmson \& Pflug (1953, S. 61, Taf. 4, Fig. 13; H. Weyland \& G. Greifeld, 1953, S. 43, Taf. 11, Fig. 48-50, 53) ist große Form. Die Bastionen sind ca. $1 \mu$ breit. Cicatricososporites virgatus Pflug (P. W. Thomson \& H. D. Pflug, 1953, S. 61, Taf. 4, Fig. 14) ist groß und besitzt feine canaliculate Skulptur.

I. C. Cookson (1957) hat Ephedra notensis Cookson (S. 45, Taf. 9, Fig. 6-10) aus den eozänen Schichten bei Frome-See, Südaustralien und Schizaea digitatoides Cookson (S. 44, Taf. 9, Fig. 1-2) aus den pliozänen Kohlen von Papua beschrieben.

N. A. Bolkhovitina hat Ephedripites mediolobatus Bolkhovitina (1953, S. 60, Taf. 9, Fig. 15-17) aus den unterkretazeischen Tonen (Hauteriv) von West-Karakhstan beschrieben. Später hat sie (1961) diese Spezies als Schizaea certa (Bolkhovitina) Bolk. mitgeteilt. Dabei hat sie drei folgende ähnliche Spezies beschrieben: Schizaea laevigataeformis BolkhovitiNa (1961, S. 29-30, Taf. 6, Fig. 1a-e; Alb-Cenoman, Senon), Schizaea evidens Bolkhovitina (1961, S. 30-31, Taf. 6, Fig. 2a-d; Neokom-Apt) und Schizaea kulandyensis Bolkноvitina (1961, S. 31, Taf. 6, Fig. 3a-k; Alb, Cenoman, Turon). Die laevigataeformis-Form ist etwas größer als die der japanischen Form und die Rippen der ersteren sind $3 \mu$ breit und 5 bis 6 mal breiter als die der letzteren. Die Rippen der evidens-Form ist etwas zweimal breiter als die der ellipsoideus-Form. Die kulandyensis-Form ist kleiner als die der ellipsoideus-Form und die Rippen der ersteren sind 3 bis 4 mal breiter als die der letzteren.

Botanische Zugehörigkeit: Schizaeaceae?

\section{? Cicatricososporites sp.}

Taf. 31, Fig. 10.

Monolete Spore (?). $24.2 \mu$ lang und $10 \mu$ breit. Figura lang-ellipsoidisch. 
Dehiszenz ist nicht zu erkennen. Exine mit einander parallelen, schmalen, etwas welligen Rippen, d. h. cicatricos. Rippen konvergieren \pm in Richtung der Schmalseiten des Äquators.

Diese kleine Form wurde nur im Kohlenflöz der unteren Hakobuchi-Schichten (Campan) von Hatsune-Sawa, Yubari-Kohlenfeld gefunden. Sie ist Schizaea kulandyensis Bolkhovitina (1961, S. 31, Taf. 6, Fig. 3a-k; Alb, Cenoman, Turon) ähnlich, aber sie kann nach Breite der Rippen von der letzteren unterschieden werden.

Botanische Zugehöorigkeit: Schizaeaceae?

Incertae Sedis

Formgattung: Schizosporis Cookson \& DeTtManN

1959. Schizosporis Cookson \& Dettmann, S. 213.

Schizosporis sp.

Taf. 31, Fig. 11a, b.

Ưber $100 \mu$ groß. Biconvex. Figura ursprünglich ellipsoidisch. Äquatorkontur elliptisch. Polkappenkontur mehr oder weniger zugespitzt. Exine $2 \mu$ dick, etwas rundliches bis polygonales Netz. Netzlumen bis $3 \mu$ breit. Spore reißt leicht in zwei Teile ein.

Nur zwei Stücke dieser Spore wurde bisher im Kohlenflöz der unteren Hakobuchi-Schichtengruppe (Campan) von Hatsune-Sawa, Yubari-Kohlenfeld gefunden.

I. C. Cookson und M. E. Dettmann (1959) haben die neue Formgattung Schizosporis (S. 213) vorgeschlagen. Dabei haben sie morphologisch ähnliche Form, Schizosporis reticulatus Cookson \& DetTmann (1959, S. 213-214, Taf. 1, Fig. 1-4), aus den ober- bis unterkretazeischen Schichten (Apt-Cenoman), Südaustralien beschrieben. Die Exine dieser Spezies ist viel dicker als die der japanischen Form und zweischichtig.

Botanische Zugehörigkeit: fraglich.

\section{Pollenites}

Aletes

Formgattung: Inaperturopollenites THomson \& PFLUG

1953. Inaperturopollenites Thomson \& PfLUG, S. 64.

Wie S. MANUM (1962) geschrieben hat, hat die Gattung Inaperturopollenites Thomson \& Pflug die Priorität. Laricoidites und Taxodiaceaepollenites waren ungültig gewesen, bis R. PotoniÉ (1958) beschrieben hat. 


\section{Inaperturopollenites pseudodubius TAKAHASHI}

Taf. 33, Fig. 16-24; Taf. 41, Fig. 5.

1957. Inaperturopollenites pseudodubius, K. TAAHASHI, S. 216, Taf. 38, Fig. 11-17; Taf. 39, Fig. 13-14.

1961. Inaperturopollenites pseudodubius, K. Takahashi, S. 295, Taf. 17, Fig. 1-7.

Die vorliegenden Figuren wurden in der oberen und unteren HakobuchiSchichtengruppe von Ooyubari, Yubari-Kohlenfeld (Hokkaido) gefunden.

Sie sind der mitteleuropäischen tertiären Spezies, Inaperturopollenites dubius (R. POT. \& VEN.) THOMSON \& PFLUG (Pollenites magnus forma dubius R. Pot. \& Ven., 1934, S. 17, Taf. 2, Fig. 20-21; P. W. Thomson \& H. D. Pflug, 1953, S. 65, Taf 4, Fig. 89; Taf. 5, Fig. 1-13), sehr ähnlich. Die pseudodubiusForm kann nach Größe, Exinenstärke u. a. von der dubius-Form unterschieden werden.

Die vorliegende Form wurde bisher am häufigsten im Paläogen und Miozän von Westjapan gefunden. Sie wird wahrscheinlich mit dem Kieselholzrest Taxodioxylon sequoianum GoTHAN in enger Beziehung stehen.

Botanische Zugehörigkeit: Taxodiaceae, Cupressaceae u. a.

\section{Inaperturopollenites magnus (R. Рот.) THOMsoN \& PFLUG \\ Taf. 31, Fig. 12 ; Taf. 41, Fig. 1.}

1931. Sporonites (?) magnus, R. Potonit, S. 556, Fig. 6.

1934. Pollenites magnus, R. Potonit, S. 48, Taf. 6, Fig. 5.

1937. Larix-pollenites magnus, RAATZ, S. 15.

1950. Laricoidites magnus, Potonie, Thomson \& Thiergart, S. 48, Taf. C, Fig. 9, 10.

1951. Laricoipollenites magnus, R. PотоNIE, Taf. 20, Fig. 26.

1953. Inaperturopollenites magnus, Thомson \& PfLUG, S. 64-65, Taf. 4, Fig. 83-88.

1955. Laevigatasporites magnus, DeLCOURT \& SPRUMONT, S. 49.

Die betreffenden Figuren wurden in der oberen und unteren HakobuchiSchichtengruppe von Ooyubari, Yubari-Kohlenfeld gefunden. Die magnusForm wurde von R. PotoNiÉ (1934) aus den eozänen Schichten von dem Geiseltal (Deutschland) erstmals beschrieben. Im westjapanischen Tertiär wurde sie bisher nie mitgeteilt.

64-82 $\mu$ groß. Figura ursprünglich kugelig. Exine zweischichtig, $1.4 \mu$ dick, sekundär verfaltet, glatt bis schwach chagrenat.

Die magnus-Form wurde bisher in den mitteleuropäischen tertiären Schichten gefunden und H. WeYland \& W. KRIEgER (1953, S. 13, Taf. 3, Fig. 12) haben dieselbe aus dem Aachener Sand (Mittelsenon) mitgeteilt und abgebildet.

G. E. Rouse (1962) hat ähnliche Form, Magnosporites staplinii RousE (S. 210, Taf. 5, Fig. 23-24), aus den eozänen Burrard-Schichten von WestBritish Columbia (Westkanada) beschrieben. Diese staplinii-Form ist größer $(100-170 \mu)$ als die magnus-Form.

Botanische Zugehörigkeit: Larix und Pseudotsuga kommen in Frage. 


\section{Inaperturopollenites (?) falsus $\mathrm{n}$. $\mathrm{sp}$.}

Taf. 41, Fig. 2, 3.

D i a g n o s e : Ca. 52-53.5 $\mu$ groß. Figura ursprünglich kugelig (?). Kontur dreieckig mit abgerundeten Ecken. Exine dünn, sekundär stark verfaltet, glatt.

H o l o ty p u s : Ca. $52 \mu$ groß; Taf. 41, Fig. 3 ; Präparat GK-V 3249.

Locus typicus: Bei der Talspere, Ooyubari, Yubari-Kohlenfeld, Hokkaido.

Stratgraphisches Verhalten: Diese Form wurde bisher nur in den Sandsteinen der oberen Hakobuchi-Schichtengruppe (Maastricht) bei der Talspere von Ooyubari, Yubari-Kohlenfeld (Hokkaido) gefunden.

Vergleich: Die betreffende Form ist Inaperturopollenites magnus (R. Potonié) Thomson \& Pflug (Pollenites magnus R. Potoní́, S. 48, Taf. 6, Fig. 5; P. W. Thomson \& H. D. Pflug, 1953, S. 64-65, Taf. 4, Fig. 83-88) ähnlich, aber die Exine der ersteren ist dünner als die der magnus-Form.

Botanische Zugehörigkeit: fraglich.

\section{Inaperturopollenites sp.}

Taf. 41, Fig. 6, 7.

Pollen im Tetradenverband, ohne Germinalapparat. Ein Pollenkorn ist 19 bis $20.2 \mu$ groß. Figura kugelig. Exine dünn, chagrenat.

Diese Pollenform im Tetradenverband wurde bisher nur im Sandstein der oberen Hakobuchi-Schichten bei der Talspere von Ooyubari, Yubari-Kohlenfeld (Hokkaido) gefunden.

Sie hat gleiche Eigentümlichkeit mit Inaperturopollenites pseudodubius TAKAHASHI, aber der Verfasser teilt wegen des Tetradenverbandes sie als Inaperturopollenites sp. mit.

Botanische Zugehörigkeit: Taxodiaceae (?)

$$
\begin{gathered}
\text { Inaperturopollenites parviundulatus n. sp. } \\
\text { Taf. 41, Fig. 9-12. }
\end{gathered}
$$

D i a g n o s e : 20-25 $\mu$ groß. Kontur vier- bis fünfeckig mit abgerundeten Ecken. Exine dünn, unduliert (d. h. rugulat), mehr oder weniger sekundär verfaltet. Ohne Germinalapparat.

H o loty pus : Ca. $22 \mu$ groß; Taf. 41, Fig. 11a, b; Präparat GK-V 3247.

Locus typicus: Bei der Talspere von Ooyubari, Yubari-Kohlenfeld, Hokkaido.

Stratigraphisches Verhalten: Diese Form wurde bisher nur im Sandstein der oberen Hakobuchi-Schichtengruppe bei der Talspere von Ooyubari, Yubari-Kohlenfeld (Hokkaido) gefunden.

Vergleich: H. Weyland und G. Greifeld (1953) haben morphologisch ähnliche Formen, Inaperturopollenites globulus H. WEYLAND \& G. GREI- 
FELD (S. 44, Taf. 13, Fig. 83-84), Inaperturopollenites parvoglobulus H. WEYLAND \& G. GReifeld (S. 44, Taf. 13, Fig. 85-86) und Inaperturopollenites undulatus H. Weyland \& G. GReifeld (S. 44, Taf. 13, Fig. 89-90), aus den untersenonen Tonen von Quedlinburg (Deutschland) beschrieben. Die globulus-Form ist größer als die parviundulatus-Form und die Exine der ersteren ist etwas unregelmäßig warzig. Die Randkrause der parvoglobulus-Form ist etwa $2 \mu$ breit. Die undulatus-Form ist rundlich und größer als die parviundulatus Form.

Botanische Zugehörigkeit: fraglich.

\section{Saccites \\ Formgattung: Pityosporites (SEWARd) MANUM}

1914. Pityosporites SEWARD, S. 23, pro parte.

1954. Pityosporites (SEW.) Potoní \& KLAUS, S. 534.

1958. Pinuspollenites RaAtz ex R. Potonit, S. 62.

1960. Pityosporites (SEw.) ex Manum, S. 14-15.

\section{Pityosporites aliformis n. sp.}

Taf. 34, Fig. 1, 2, 4.

D i a g n o s e : $73-85 \mu$ groß. Luftsäcke unterhalbkugelig, kleiner als das Mittelkorn. Längster Durchmesser der Luftsäcke 40 bis $50 \mu$. Kein oder schmaler (unter $2 \mu$ ) Kamm, intrarugulat. Stäbchen in den Luftsäcken chagrenat bis reticulat geordnet. Stäbchen im Korn chagrenat bis rugulat angeordnet.

H o l o t y p u s : Ca. $85 \mu$ groß; Taf. 34, Fig. 4; Präparat GK-V 3143.

L ocus typicus: Hatsune-Sawa, Ooyubari, Yubari-Kohlenfeld, Hokkaido.

Stratigraphisches Verhalten: Diese Spezies wurde bisher im Kohlenflöz der unteren Hakobuchi-Schichtengruppe (Campan) von HatsuneSawa, Yubari-Kohlenfeld (Hokkaido) gefunden.

Vergleich: Diese Form ist der mitteleuropäischen jungtertiären Spezies, Pityosporites labdacus (R. Рот.) Thomson \& PFLUG (Pollenites labdacus PotoniÉ, 1931, S. 5, Fig. 32; Abietineae-pollenites labdacus Potoní́, 1951, S. 144, Taf. 20, Fig. 22, 23; Pinuspollenites labdacus Ротоní́, S. 62; Thomson \& Pflug, 1953, S. 68, Taf. 5, Fig. 60-62) ähnlich. Die Netzlumen der Luftsäcke von labdacus-Form sind größer als die der aliformis-Form.

E. D. Zaklinskaja (1957) hat morphologisch ähnliche Form, Pinus taedaeformis ZAKLINSKAJA (S. 156, Taf. 14, Fig. 5-11), aus den eozänen Schichten im Nord-Aralgebiet, Rußland beschreiben. Die betreffende aliformis-Form kann nach Stärke des Kamms von der taedaeformis-Form unterschieden werden.

Botanische Zugehörigkeit: Pinus.

Pityosporites microaliformis n. sp.

Taf. 35, Fig. 3, 5, 6 .

D i a g n o s e : Ca. 54-61 $\mu$ groß. Luftsäcke unterhalbkugelig. Längster 
Durchmesser der Luftsäcke 30 bis $37 \mu$. Kein oder sehr schmaler Kamm. Stäbchen in den Luftsäcken chagrenat geordnet und Stäbchen im Mittelkorn chagrenat geordnet.

H o l o ty p u s : $61 \mu$ groß; Taf. 35, Fig. 3 ; Präparat GK-V 3132.

L ocus typicus: Hatsune-Sawa, Ooyubari, Yubari-Kohlenfeld, Hokkaido.

Stratigraphisches Verhalten: Diese Spezies fand sich bisher im Kohlenflöz der unteren Hakobuchi-Schichtengruppe (Campan) von Hatsune-Sawa, Yubari-Kohlenfeld (Hokkaido).

Vergle ich: Die vorliegende Form ist Pityosporites aliformis TAKAHASHI (in dieser Arbeit, Taf. 34, Fig. 1, 2, 4) ähnlich, aber die erstere ist kleiner als die letztere. Die microaliformis-Form ist auch der mitteleuropäischen tertiären Spezies, Pityosporites labdacus (R. Рот.) Thомson \& Pflug, ähnlich. Die Exine der ersteren Luftsäcke ist chagrenat, aber die der letzteren reticulat.

Botanische Zugehörigkeit: Wahrscheinlich Pinus.

Pityosporites sp.

Taf. 34, Fig. 5a, b.

Über $110 \mu$ groß. Mittelkorn ca. $84 \mu$ lang. Längster Durchmesser der Luftsäcke ca. $56 \mu$. Luftsäcke unterhalbkugelig. Kamm stark, $3.2 \mu$ dick, intrarugulat. Stäbchen in den Luftsäcken fein reticulat geordnet. Stäbchen im Korn chagrenat bis rugulat geordnet.

Diese große Spezies fand sich bisher nur im Kohlenflöz der unteren Hakobuchi-Schichtengruppe (Campan) von Hatsune-Sawa im Yubari-Kohlenfeld (Hokkaido).

Botanische Zugehörigkeit: Pinus.

\section{Formgattung: Piceaepollenites R. PotoniÉ}

1958. Piceaepollenites R. Potonit, S. 64.

Der Gattungsname wurde erst von R. РотоNiÉ (1931, S. 28, Taf. 2, Fig. V70a; 1931, S. 5, Fig. 31) benutzt. Dabei hat er keine Beschreibung der Gattung oder Spezies, sondern nur einige Bezeichnungen gegeben. Später hat er in R. PotoniÉ \& H. Venitz (1934, S. 18, Taf. 2, Fig. 22) beschrieben, aber er hat dabei den Gattungsnamen Piceae?-pollenites benutzt. Der Name mit einem Fragezeichen ist nach I. C. nicht gültig. Deshalb wurde der Gattung Piceaepollenites im Jahre 1958 von R. PотоNí́ zum ersten Mal gültig beschrieben.

Piceaepollenites saccellus n. sp.

Taf. 34, Fig. 3; Taf. 35; Fig. 1, 2, 7.

D i a g n os e : Ca. 49.5-69 $\mu$ groß. Luftsäcke halbkugelig. Längster 
Durchmesser der Luftsäcke 27 bis $30 \mu$. Kamm schmal, bis $2 \mu$ dick, intrarugulat bis intrabaculat. Stäbchen in den Luftsäcken chagrenat bis reticulat. Stäbchen im Korn chagrenat bis fein reticulat. Die Umrißlinie des Zentralkörpers geht ohne deutlichen Knick in die Umrißlinie der Sacci über.

H olotypus : Ca. $65 \mu$ groß; Taf. 35; Fig. 1; Präparat GK-V 3142.

Locus typicus: Hatsune-Sawa, Ooyubari, Yubari-Kohlenfeld, Hokkaido.

Stratigraphisches Verhalten: Diese Spezies fand sich bisher im Kohlenflöz der unteren Hakobuchi-Schichtengruppe (Campan) von HatsuneSawa, Yubari-Kohlenfeld, Hokkaido.

Vergle ich: Die betreffende Spezies ist der mitteleuropäischen tertiären Art, Piceaepollenites alatus R. Рот. (Piceae?-Pollenites alatus R. Рот. \& H. Ven., 1934, S. 18, Taf. 2, Fig. 12; Pityospor. alatus (R. PoT.) Thomson \& Pflug, 1953, S. 68, Taf. 5, Fig 63, 65, 66) ähnlich. Das Stäbchen im Korn der letzteren ist fein rugulat.

G. E. Rouse (1957) hat Picea grandivescipites WoDEhouse (S. 368, Taf. 2, Fig. 11, 12) aus den oberkretazeischen Oldman-Schichten (Campan) von Südalberta, Westkanada mitgeteilt.

N. A. Bolkhovitina (1956) hat ähnliche Spezies, Picea complanatiformis Bolkhovitina (S. 103, Taf. 17, Fig. 187) aus den unterkretazeischen Schichten von Yakutsk, A. S. S. R., Rußland beschrieben. Diese Form ist größer als die saccellus-Form.

Botanische Zugehörigkeit: Picea.

\section{Formgattung: Podocarpidites (CoOKsON) R. POTONIÉ}

1947. Podocarpidites Cookson, S. 131.

1958. Podocarpidites (Cookson) R. Potonik, S. 68.

\section{Podocarpidites sp. a}

Taf. 35, Fig. 4.

$80 \mu$ groß. Längster Durchmesser des Korns $30 \mu$. Längster Durchmesser der Luftsäcke $45 \mu$. Kamm ca. $2.5 \mu$ dick. Kontur der Luftsäcke elliptisch. Exine im Korn und in den Luftsäcken chagrenat.

Diese Form wurde bisher nur im Kohlenflöz der unteren HakobuchiSchichtengruppe (Campan) von Hatsune-Sawa, Yubari-Kohlenfeld (Hokkaido) gefunden.

Botanische Zugehörigkeit: Podocarpaceae?

\section{Podocarpidites sp. $\mathrm{b}$}

Taf. 35, Fig. 8.

Über $50 \mu$ groß. Längster Durchmesser der Luftsäcke ca. $40 \mu$. Das Korn ist kleiner als der Luftsack. Kontur der Luftsäcke rundlich. Stäbchen der Luftsäcke reticulat (?) geordnet. 
Diese Form fand sich bisher nur im Kohlenflöz der unteren HakobuchiSchichtengruppe (Campan) von Hatsune-Sawa, Yubari-Kohlenfeld, Hokkaido.

S. SAto (1961) hat Podocarpidites (al. Podocarpus) ezoensis (SATo) (S. 89, Taf. 1, Fig. 29-30) aus den oberkretazeischen Hakobuchi-Schichten in Hokkaido beschrieben.

I. C. Cookson (1947) hat Podocarpidites elliptica Cookson (S. 131, Taf. 13, Fig. 5-7) und Podocarpidites microreticuloidata Cookson (S. 131, Taf. 13, Fig. 8-11) aus den tertiären Braunkohlen und karbonreichen Sandsteinen von der Kerguelen Insel beschrieben.

R. A. Couper (1953) hat Podocarpidites marwickii Couper (S. 36, Taf. 4, Fig. 39; Kreide), Podocarpidites major Couper (S. 36, Taf. 4, Fig. 40; Jura), Podocarpidites otagoensis Couper (S. 36, Taf. 4, Fig. 41; Oberkreide) u. a. aus Neuseeland beschrieben

G. E. Rouse (1957) hat Podocarpidites biformis Rouse (S. 367, Taf. 2, Fig. 13) aus den oberkretazeischen Oldman-Schichten von Südalberta (Westkanada) beschrieben.

E. D. Zaklinskaja (1957) hat Podocarpidites (al. Porlocarpus) sellowiformis (Zaklinskaja) (S. 104, Taf. 2, Fig. 2; Oberkreide, Pavlodar Irtysh Becken), Podocarpilites (al. Podocarpus) andiniformis (ZAKLINSKAJA) (S. 105, Taf. 2, Fig. 3-7; Oberkreide, Pavlodar Irtysh Becken), Podocarpidites (al. Podocarpus) nageiaformis (Zaklinskaja) (S. 106, Taf. 2, Fig. 8-11; Unteroligozän, Pavlodar Irtysh Becken), Podocarpidites (al. Podocarpites) kasakhstanica (ZaklinskaJA) (S. 108, Taf. 2, Fig. 12-15; Mitteloligozän, Pavlodar Irtysh Becken) und Podocarpidites (al. Podocarpites) gigantea (ZAKLinskaja) (S. 109, Taf. 3, Fig. 1-4; Oberoligozän, Pavlodar Irtysh Becken) beschrieben.

Botanische Zugehörigkeit: Podocarpus.

Formgattung: Phyllocladidites (COOKSON) COUPER

1958. Phyllocladidites (CooKson) CouPER 1953, in R. PoToní́, S. 69-70.

Phylloclaridites brachypterus n. sp.

Taf. 35, Fig. 9, 10, 14.

D i a g n o s e : Ca. 36-36.5 $\mu$ groß. Kontur in der Polansicht kreisförmig. Zwei Luftsäcke (Sacci) klein, halbkreisförmig, wohl weit distal vom Äquator stehend (?). Exine chagrenat.

Die Figur 14 gehört zu dieser Spezies sehr zweifelhaft.

H o loty pu s : Ca. $36 \mu$ groß; Taf. 35, Fig. 9; Präparat GK-V 3132.

Locus typicus: Hatsune-Sawa, Ooyubari, Yubari-Kohlenfeld, Hokkaido.

Stratigraphisches Verhalten: Diese Spezies wurde bisher nur im Kohlenflöz der unteren Hakobuchi-Schichtengruppe (Campan) von Hatsune-Sawa, Yubari-Kohlenfeld, Hokkaido gefunden. 
Vergleich: I. C. Cookson hat ähnliche Formen, Phyllocladidites mawsoni (CooKsoN) [Disaccites (Phyllocladidites) mawsoni CooKsoN 1947, S. 133, Taf. 14, Fig. 22-28; Tertiär, Kerguelen Insel; Dacrydiumites mawsoni Cookson, 1953, S. 465, Taf. 1, Fig. 10; Alttertiär, Comaum, S. Australien] und Phyllocladidites rüei (CooKson) [Disaccites (Phyllocladidites) rüei Cookson, 1947, S. 133, Taf. 13, Fig. 31-37; Tertiär, Kerguelen Insel] beschrieben.

R. A. COUPER (1958) hat die neue Gattung Parvisaccites für die ähnliche Form mit den kleinen Luftsäcken vorgeschlagen. Dabei hat er Parvisaccites radiatus Couper (S. 154, Taf. 29, Fig. 5-8; Taf. 30, Fig. 1, 2) aus den Wealden von England und Parvisaccites euigmatus Couper (S. 154-155, Taf. 30, Fig. 3-5) aus dem Mitteljura von Yorkshire beschrieben.

S. Sato (1961) hat morphologisch ähnliche Formen, Dacrydium ? transiens SAto (S. 89-90, Taf. 1, Fig. 23-24) und Macrozamia? hakobuchiensis SATo (S. 90, Taf. 1, Fig. 22), aus den oberkretazeischen Osoushinai-Schichten in Nordhokkaido beschrieben. Besonders ist Macrozamia ? hakobuchiensis SATo der vorliegenden Form sehr ähnlich.

Botanische Zugehörigkeit: Dacrydium?

\section{Phyllocladidites mirandus n. sp. Taf. 35, Fig. 11-13.}

D i a g n o s e : Ca. 35.5-38 $\mu$ groß. Kontur der Polansicht breit-elliptisch. Zwei Luftsäcke klein, halskrauseartig (3.5 bis $4.5 \mu$ breit), gegeneinander in weiten Abstand stehend. Exine bis $1.5 \mu$ dick, chagrenat.

Die Figuren 11a und $11 \mathrm{~b}$ gehören zu dieser Spezies zweifelhaft.

H o loty p u s : $38 \mu$ groß; Taf. 35, Fig. 13; Präparat GK-V 3152.

Locus typicus: Hatsune-Sawa, Ooyubari, Yubari-Kohlenfeld, Hokkaido.

Stratigraphisches Verhalten: Die vorliegende Form fand sich nur im Kohlenflöz der unteren Hakobuchi-Schichtengruppe (Campan) von Hatsune-Sawa, Yubari-Kohlenfeld, Hokkaido.

Vergle i ch: Die betreffende Form ist Phyllocladidites mawsoni (CookSON) und Phyllocladidites rïei (CoOKsoN) ähnlich. Die erstere kann nach Form des Luftsacks und Dicke der Exine von der mawsoni-Form und nach Größe des Korns und Skulptur der Exine von der rïei-Form unterschieden werden.

Dacrydium ? transiens SATo (1961) ist der vorliegenden mirandus-Form morphologisch ähnlich, aber die letztere kann nach Form des Korns, Skulptur der Exine und Breite des Luftsacks von der transiens-Form unterschieden werden.

Botanische Zugehörigkeit: Dacrydium? 
Poroses

Normapolles

Tirporines

Formgattung: Trudopollis PfLUG

1953. Trudopollis Pflug, S. 98.

? Trudopollis sp.

Taf. 42, Fig. 15.

Ca. $33 \mu$ groß. Kontur gebrochen konkav, Innenkontur konvex dreieckig. Germinalien bilden etwas lange Schnäbel. Porenkanalindex ca. 0.16. Poren äquatorial. Exine verhältnismäßig dünn. Interloculum?

Es ist sehr zweifelhaft, daß diese Form zu Trudopollis gehört, da das Interloculum fraglich vorhanden ist.

Botanische Zugehörigkeit: fraglich.

\section{Postnormapolles}

Triporines

Formgattung: Momipites WoDEHouse

1933. Momipites WoDEHOUSE, S. 511.

P. W. Thomson und H. D. Pflug (1953, S. 82) haben die Formgattung Triporopollenites für dreieckige Pollen mit dreieckigem oder rundlichem Äquator und eckenständigen, äquatorial gelegenen Poren vorgeschlagen. Die Formgattung Momipites ist nur für den kleinen Teil davon gültig.

Momipites constatus (TAKAHASHI) n. comb.

Taf. 36, Fig. 15, 16.

1961. Triporopollenites constatus, K. TAKAHASHI, S. 300-301, Taf. 19, Fig. 19-28.

Ca. 18-37 $\mu$ groß. Kontur dreieckig mit konvexen Seiten. Keinerlei Anulusbildung. Keine Tumeszens. Zuweilen sehr schwaches Labrum. Poren kreisrund. Exoporus größer als der Endoporus. Exine sehr dünn, unter $1 \mu$ dick, allseits fast gleich dick, chagrenat bis fein punctat.

Fig. 15 ist ca. $37 \mu$ groß. Fig. 16 ist ca. $31 \mu$ groß. Die constatus-Form wurde bisher nur im Tertiär von Japan gefunden, aber sie tritt in der Oberkreide von Hokkaido wenig auf.

Botanische Zugehörigkeit: Betulaceae. 
Ca. $29 \mu$ groß. Kontur dreieckig mit konvexen Seiten. Keine Anulusbildung. Keine Tumeszenz. Schwaches Labrum. Exine sehr dünn, chagrenat.

Diese Form wurde im Sandstein der oberen Hakobuchi-Schichten bei der Talspere von Ooyubari im Yubari-Kohlenfeld, Hokkaido gefunden. Sie kann hier nur als Momipites sp. bezeichnet werden. Sie ist Momipites constatus (TAKAHASHI) morphologisch näherstehend.

Botanische Zugehörigkeit: Betulaceae.

Formgattung: Triporopollenites Thomson \& PFLUG

1953. Triporopollenites Thomson \& Pflug, S. 82.

\section{Triporopollenites festatus TAKAHASHI}

Taf. 36, Fig. 17.

1961. Triporopollenites festatus, K. TAKahashI, S. 301, Taf. 19, Fig. 29-37.

Fig. 17 ist ca. $32 \mu$ groß. Kontur dreieckig mit konvexen Seiten. Tropfenförmige Anulusbildung. Schwaches Labrum. Exine dünn, chagrenat.

Die festatus-Form wurde bisher im Tertiär von Japan gefunden. In der Oberkreide von Hokkaido tritt sie selten auf.

Die betreffende Figur wurde im Kohlenfiöz der unteren Hakobuchi-Schichten von Hatsune-Sawa im Yubari-Kohlenfeld gefunden.

Botanische Zugehörigkeit: Betulaceae.

\section{Triporopollenites kasuyaensis TAKAHASHI}

Fig. 36, Fig. 18.

1961. Triporopollenites kasuyaensis, K. TAKahashI, S. 303-304, Taf. 19. Fig. 40, 45; Taf. 20, Fig. 1.

Fig. 18 ist ca. $25 \mu$ groß. Kontur dreieckig mit konvexen Seiten. Großes Labrum. Keine Anulusbildung. Keine Tumeszenz. Exine sehr dünn, chagrenat.

Die kasuyaensis-Form wurde bisher in den Sanjaku-Goshaku- und OngaSchichten des Chikuho-Kohlenfeldes, in den Onga-Schichten des Kokura-Kohlenfeldes, in den Takada- und Shinbaru-Schichten des Kasuya-Kohlenfeldes, in den Yoshinotani- und Kyuragi-Schichten des Karatsu-Kohlenfeldes und in den Yunoki-Schichten des Sasebo-Kohlenfeldes gefunden, nämlich sie trat im Alttertiär und Miozän von Westjapan selten auf. Sehr selten wurde sie auch in der Oberkreide von Hokkaido gefunden.

Botanische Zugehörigkeit: Vielleicht Betulaceae.

\section{? Triporopollenites sp. \\ Taf. 42, Fig. 16.}

$25.5 \mu$ groß. Kontur elliptisch. Keine Anulusbildung. Keine Tumeszenz. Kein Labrum. Pore liegt subäquator (?). Exine dünn, chagrenat. 
Diese Form wurde im Sandstein der oberen Hakobuchi-Schichtengruppe bei der Talspere von Ooyubari im Yubari-Kohlenfeld, Hokkaido gefunden. Da sie nicht gut erhalten ist, kann der Verfasser nur als ? Triporopollenites sp. bezeichen.

Botanische Zugehorigkeit: Betulaceae?

\section{Formgattung: Trivestibulopollenites PFLUG}

1953. Trivestibulopollenites Pflug in Thomson \& Pflug, S. 84.

\section{Trivestibulopollenites sp. \\ Taf. 42, Fig. 9a, b.}

Ca. $26 \mu$ groß. Kontur dreieckig mit konvexen Seiten und prominentem Labrum. Kleines Vestibulum. Exine schwach glatt, $0.7 \mu$ dick.

Nur ein Korn dieser Form wurde bisher im Sandstein der oberen Hakobuchi-Schichtengruppe bei der Talspere von Ooyubari im Yubari-Kohlenfeld, Hokkaido gefunden.

Im Alttertiär von Westjapan wurde nur Trivestibulopollenites sp. vom Verfasser (1961) aus den Ube-Schichten (Eozän) mitgeteilt. Die betreffende Form steht Triporates, Typus A aus dem Tertiär von Spitzbergen (S. MANUM, 1962) morphologisch am nächsten.

Botanische Zugehörigkeit: Betulaceae.

Formgattung: Betulaepollenites R. PotoniÉ

1934. Betulaepollenites R. Potoní́, S. 58.

1960. Betulaepollenites R. Potoní, S. 115.

R. Potoní́ (1931) hat Pollenites microexcelsus mit einigen Bezeichnungen des morphologischen Charakters mitgeteilt. Später hat er (1934) denselben als Betulaepollenites microexcelsus monotypisch beschriebon.

Betulaepollenites normalis $\mathrm{n}$. sp.

Taf. 36, Fig. 20-27; Taf. 42, Fig. 14.

D i a g n o s e : Ca. $22-34 \mu$ groß. Kontur dreieckig mit konvexen Seiten. Poren groß, kreisrund, meridional nicht tief. Keine Anulusbildung. Großes Labrum. Arci stark ausgeprägt; an der Polansicht verlaufen die Arci etwas bogenlinig von Exitus zu Exitus. Exine bis $1.5 \mu$ dick, chagrenat.

H o l o t y p u s : $32.5 \mu$ groß; Taf. 36, Fig. 22a, b; Präparat GK-V 3131.

Locus typicus: Hatsune-Sawa, Ooyubari, Yubari-Kohlenfeld, Hokkaido.

Stratigraphisches Verhalten: Diese neue Spezies wurde im Kohlenflöz der unteren Hakobuchi-Schichtengruppe von Hatsune-Sawa und im Sandstein der oberen Hakobuchi-Schichten bei der Talspere von Ooyubari, Hokkaido gefunden.

Vergleich: Die normalis-Form steht Betulaepollenites microexcelsus 
Potonié (Pollenites microexcelsus R. Potonié, 1931, S. 3, Fig. 8; R. Potonié, 1934, S. 58, Taf. 2, Fig. 22, 27 ; R. Potonié \& H. Venitz, 1934, S. 25, Taf. 2, Fig. 50) aus dem miozänen und eozänen Humodil der niederrheinischen Bucht und des Geiseltals morphologisch am nächsten. Die erstere kann nach Größe von der letzteren unterschieden werden.

S. MANUM (1962) hat morphologisch ähnliche Form als Triporates, Typus E (S. 60-61, Taf. 15, Fig. 18-21) aus dem Tertiär von Spitzbergen beschrieben.

Botanische Zugehörigkeit: Betula-Typus.

\section{Betulaepollenites minutulus n. sp. \\ Taf. 42, Fig. 1-7.}

D i a $\mathrm{g}$ o s e : $16.8-21.7 \mu$ groß. Kontur dreieckig mit konvexen Seiten. Poren kreisrund, meridional nicht tief. Meistens keine Anulusbildung, selten schwach tropfenförmig. Meistens kein Labrum, selten sehr schwaches. Arci stark ausgeprägt; an der Polansicht verlaufen die Arci etwas geradlinig von Exitus zu Exitus. Exine dünn, chagrenat.

H o l o ty p us : $16.8 \mu$ groß; Taf. 42, Fig. 1a, b; Präparat GK-V 3215.

Locus typicus: Bei der Talspere von Ooyubari, Yubari-Kohlenfeld, Hokkaido.

Stratigraphisches Verhalten: Die betreffende Form wurde in den Sandsteinen der oberen Hakobuchi-Schichtengruppe (Maastricht) beschränkt gefunden.

Vergle i ch: Die minutulus-Form ist der mitteleuropäischen tertiären Spezies Betulaepollenites microexcelsus R. PоTоNIÉ (Pollenites microexcelsus R. Potonié, 1931, S. 3, Fig. 8; R. РотоNiÉ, 1934, S. 58, Taf. 2, Fig. 22, 27 ; R. Potonié \& H. Venitz, 1934, S. 25, Taf. 2, Fig. 50) und der oberkretazeischen Form Betulaepollenites normalis Takahashi (in dieser Arbeit, S. 231, Taf. 36, Fig. 20-27; Taf. 42, Fig. 14) morphologisch ähnlich. Die erstere kann nach Labrum von der microexcelsus-Form und nach Größe und Labrum von der normalis-Form unterschieden werden.

Botanische Zugehörigkeit: Betula-Typus.

\section{Betulaepollenites sp.}

Taf. 42, Fig. 8.

Ca. $25 \mu$ groß. Kontur dreieckig mit konvexen Seiten und abgerundeten Ecken. Poren kreisrund, meridional nicht tief. Keine Anulusbildung. Kein Labrum. Keine Tumeszenz. Atrium? Arci verlaufen geradlinig von Exitus zu Exitus. Exine dünn, chagrenat.

Diese Form wurde im Sandstein der oberen Hakobuchi-Schichten bei der Talspere von Ooyubari im Yubari-Kohlenfeld, Hokkaido gefunden.

Sie kann nach Größe von der minutulus-Form und nach Labrum und Form von der normalis-Form unterschieden werden. Der Verfasser kann sie nur als Betulaepollenites sp. bezeichnen. 
Botanische Zugehörigkeit: Betula-Typus.

Formgattung: Subtriporopollenites Thomson \& PFLUG

1953. Subtriporopollenites Thomson \& Pflug, S. 85.

R. PotoniÉ und H. Venitz (1934) hat die Formgattung Caryae?-pollenites vorgeschlagen und beschrieben. Nach I. C. ist der Gattungsname mit dem Fragezeichen nicht gültig. G. V. RAATz (1937) hat den Formgattungsnamen Caryapollenites benutzt, aber er hat ihn nicht difiniert. Deshalb ist die Formgattung Subtriporopollenites THOMSON \& PFLUG nomenklatorisch gültig.

Subtriporopollenites kyushuensis TAKAHASHI

Taf. 36, Fig. 19.

1961. Subtriporopollenites kyushuensis, K. TAKahashi, S. 305-306, Taf. 20, Fig. 3537; Taf. 21, Fig. 1-10.

Ca. $34.5 \mu$ groß. Kontur kreisrund. Poren rundlich bis elliptisch; zwei Poren subäquatorial, eine Pore äquatorial. Exine sehr dünn, chagrenat. Ohne Labrum und Anulus. Sekundär verfaltet.

Die vorliegende Form fand sich im Kohlenflöz der unteren HakobuchiSchichtengruppe von Hatsune-Sawa im Yubari-Kohlenfeld, Hokkaido.

Subtriporopollenites kyushuensis TAKAHASHI wurde bisher im Alttertiär und Miozän von Westjapan und Nordostjapan gefunden. In der Oberkreide von Hokkaido tritt die kyushuensis-Form sehr selten auf.

Botanische Zugehörigkeit: Carya?

\section{Polyporines}

Formgattung: Alnipollenites R. PotoniÉ

1934. Alnipollenites R. POTONIÉ, S. 58.

1937. Alnuspollenites RAATZ, S. 20.

1953. Polyvestibulopollenites Pflug in Thomson \& Pflug, S. 90.

1960. Alnipollenites R. Potoní, S. 129-130.

R. Potoní (1931) hat Pollenites verus mitgeteilt, aber er hat keine Beschreibung gegeben. Später hat er (1934) denselben als Alnipollenites verus beschrieben. Alnuspollenites und Polyvestibulopollenites sind Synonym von Alnipollenites.

Alnipollenites eminens (TAKAHASHI) n. comb.

Taf. 36, Fig. 1-14; Taf. 41, Fig. 28-30.

1961. Polyvestibulopollenites eminens, K. Takahashi, S. 308, Taf. 20, Fig. 21-34.

1962. Alnus eminens, K. Takahashi, S. 18, Taf. 3, Fig. 33-34.

24.5-27 $\mu$ groß. Vier- bis sechsporig, meist vier- bis fünfporig. Kontur vier- bis sechseckig mit geraden bis schwach konvexen oder schwach konkaven 
Seiten. Mit Vestibulum und schwachem oder großem Labrum. Benachbarte Vestibulen kommunizieren durch paarige Schläuche (Arci), die symmetrisch zum Äquator liegen. Diese verlaufen in der Polansicht girlandenförmig und gelten als bestes Merkmal dieser Gruppe. Exine dünn, bis $1.3 \mu$ dick, chagrenat oder schwach rugulat bis fein punctat.

Die eminens-Form tritt im Tertiär von Japan sehr häufig auf. In der Oberkreide von Hokkaido tritt sie auch sehr oft auf.

Vergleichsmaterial:

Europa

Alnipollenites verus R. PotoniÉ

R. Potonié, 1934, S. 58, Taf. 2, Fig. 13, 17, 18; Eozän, Geiseltal.

R. Potonié \& H. Venitz, 1934, S. 25, Taf. 2, Fig. 51; Miozän; niederrheinische Bucht.

P. W. Thomson \& H. D. Pflug, 1953, S. 90, Taf. 10, Fig. 62-76.

Alnipollenites sp. Typus A, Typus B und Typus C.

S. Manum, 1962, S. 62-63, Typus A-Taf. 16, Fig. 1-4; Typus B-

Taf. 16, Fig. 5-9; Typus C-Taf. 16, Fig. 10; Tertiär; Spitzbergen. U. S. A.

Alnipollenites (al. Alnus) speciipites (WodEHouse)

R. P. Wodehouse, 1933, S. 508, Fig. 40; Eozän, Green River Schichten.

Kanada

Alnipollenites (al. Alnus) quinquepollenites (RoUSE)

G. E. Rouse, 1962, S. 202, Taf. 2, Fig. 7, 8; Eozän; BurrardSchichten.

Alnipollenites (al. Alnus) quadrapollenites (RoUSE)

G. E. Rouse, 1962, S. 202, Taf. 2, Fig. 9, 36; Eozän; BurrardSchichten.

Botanische Zugehörigkeit: Alnus.

Formgattung: Ulmipollenites WoLFF

1934. Ulmipollenites WoLFF, S. 75.

H. WoLfF (1934) hat Ulmipollenites undulosus WoLFF (S. 75, Taf. 5, Fig. 25) monotypisch beschrieben. H. D. Pflug (1953) hat denselben als Polyporopollenites undulosus (WOLFF) beschrieben, aber die Formgattung Ulmipollenites ist nomenklatorisch gültig.

Ulmipollenites sp.

Taf. 42, Fig. 21, 22.

25-35.5 $\mu$ groß. Vier- bis fünfporig. Kontur eckig bis rundlich. Exine sehr dünn. Feine rugulate Skulptur.

Diese Art fand sich nur in den Sandsteinen der oberen Hakobuchi-Schichten bei der Talspere von Ooyubari im Yubari-Kohlenfeld, Hokkaido. 
Der Verfasser kann hier nur sie als Ulmipollenites sp. mitteilen, da sie nicht gut erhalten ist. Sie scheint in den oberen Hakobuchi-Schichtengruppe beschränkt aufzutreten. Sie kann nach Skulptur der Exine von Ulmipollenites undulosus WoLFF leicht unterschieden werden.

Botanische Zugehörigkeit: Ulmaceae.

Polyporopollenites (PFLug, 1953) n. emend.

Ty pus: Polyporopollenites carpinoides Pflug in Thomson \& Pflug, 1953, S. 92, Taf. 10, Fig. 79, Ca. $34 \mu$.

D i a g n o s e : Gestalt oblat, Äquator polygonal. Porenzahl wechselt innerhalb einer Gruppe, normalerweise vier bis viele Poren vorhanden. Diese eckenständig, äquatorial oder subäquatorial gelegen; zuweilen Anulus, Labrum oder Atrium; ohne Vestibulum. Exine glatt bis chagrenat oder sonstig skulpturiert oder strukturiert, nie rugulat. Polachse ist Symmetrieachse entsprechend der Porenzahl. Äquatorebene ist nur Symmetrieebene, wenn die Poren äquatorial liegen.

H. D. Pflug (1953) hat die neue Formgattung Polyporopollenites vorgeschlagen, dabei hat er als Genotyp Polyporopollenites undulosus (WoLFF) festgesetzt. Aber H. WoLfF (1934) hat Ulmipollenites undulosus WoLFF als morotypisch gültig beschrieben. Deshaib muß die Formgattung Polyporopollenites emendiert beschrieben werden und ihr Geotypus muß neu festgesetzt werden, um die Formgattung Polyporopollenites nomenklatorisch gelten zu lassen. Der Verfasser verfährt das nach dem I. C.

\section{Polyporopollenites punctatus n. sp.}

Taf. 42, Fig. 19, 23, 24.

D i a g n o s e : Ca. 22-30.5 $\mu$ groß. Vier- bis fünfporig. Kontur vier- bis fünfeckig mit abgerundeten Ecken. Exine dünn, bis $1 \mu$ dick, grob punktat. Poren klein. Selten sehr schwacher Anulus. Schwaches Labrum.

H o l o ty p u s : Ca. $22 \mu$ groß; Taf. 42, Fig. 24a, b; Präparat GK-V 3249.

Locus typicus: Bei der Talspere von Ooyubari, Yubari-Kohlenfeld, Hokkaido.

Stratigraphisches Verhalten: Diese Form fand sich bisher nur in den Sandsteinen der oberen Hakobuchi-Schichtengruppe bei der Talspere von Ooyubari im Yubari-Kohlenfeld, Hokkaido. Sie scheint in den oberen Hakobuchi-Schichten beschränkt aufzutreten.

Vergleich: Die punctatus-Form ist den mitteleuropäischen ältesttertiären Spezies, Polyporopollenites validus Pflug (P. W. THomson \& H. D. Pflug, 1953, S. 91, Taf. 10, Fig. 44-51) und Polyporopollenites polyangulus Pflug (P. W. Thomson \& H. D. Pflug, 1953, S 91, Taf. 10, Fig. 59-61) ähnlich, aber die erstere kann nach Skulptur oder Struktur der Exine von den zwei letzteren unterschieden werden.

Botanische Zugehörigkeit: Ulmaceae? 


\section{Polyporopollenites sp. a. \\ Taf. 42, Fig. 13.}

Ca. $28 \mu$ groß. Vierporig. Kontur viereckig mit geraden oder schwach konvexen Seiten. Poren klein. Keine Anulusbildung. Kein Labrum. Keine Tumeszenz. Exine dünn, chagrenat.

Der betreeffnde Pollen ist nicht gut erhalten. Er wurde im Sandsteine der oberen Hakobuchi-Schichtengruppe bei der Talspere von Ooyubari im YubariKohlenfeld, Hokkaido gefunden.

Botanische Zugehörigkeit: Betulaceae?

\section{Polyporopollenites sp. b.}

Taf. 42, Fig. 17.

$28.5 \mu$ groß. Fünfporig. Kontur fünfeckig mit geraden Seiten. Poren äquatorial. Dünnwandige Exine. Schwaches Labrum. Anulus von tropfenförmigem Querschnitt. Exine schwach chagrenat.

Nur ein Pollenkorn wurde im Sandstein der oberen Hakobuchi-Schichtengruppe bei der Talspere von Ooyubari im Yubari-Kohlenfeld, Hokkaido gefunden.

Die betreffende Form kann nach Anulus- und Labrumbildung von der Polyporopollenites sp. a unterschieden werden.

\section{Polyporopollenites sp. c}

Taf. 42, Fig. 18.

Ca. $24 \mu$ groß. Vierporig(?). Kontur viereckig mit geraden oder schwach konvexen Seiten und abgerundeten Ecken. Poren klein (?) und subäquatorial (?). Keine Anulusbildung. Keine Tumeszenz. Kein Labrum. Exine dünn, chagrenat.

Diese Form wurde im Sandstein der oberen Hakobuchi-Schichten bei der Talspere von Ooyubari im Yubari-Kohlenfeld, Hokkaido gefunden. Sie ist nicht gut erhalten.

Botanische Zugehörigkeit: fraglich.

\section{Polyporopollenites sp. d.}

Taf. 42, Fig. 20.

$16.4 \mu$ groß. Vierporig. Kontur viereckig mit geraden und konvexen Seiten und abgerundeten Ecken. Keinerlei Anulusbildung. Keine Tumeszenz. Kein Labrum. Exine dünn, chagrenat. Poren klein, subäquatorial.

Nur ein Pollenkorn wurde im Sandstein der oberen Hakobuchi-Schichengruppe bei der Talspere von Ooyubari im Yubari-Kohlenfeld, Hokkaido gefunden.

Botanische Zugehörigkeit: fraglich. 


\section{Polyporopollenites sp. e. \\ Taf. 42, Fig. 25a, b.}

$30.8 \mu$ groß. Vierporig. Kontur viereckig mit konvexen Seiten und abgerundeten Ecken. Poren äquatorial. Schwaches Labrum. Keine Anulus- und Tumeszenzbildung. Exine dünn, chagrenat.

Nur ein Pollenkorn fand sich im Sandstein der oberen Hakobuchi-Schichtengruppe bei der Talspere von Ooyubari im Yubari-Kohlenfeld, Hokkaido.

Die betreffende Figur ist schlecht erhalten. Deshalb kann der Verfasser nur sie als Polyporopollenites sp. e. beschreiben.

Botanische Zugehörigkeit: fraglich.

\section{Polyporopollenites sp. f.}

Taf. 42, Fig. 26.

Ca. $27 \mu$ groß. Vierporig. Kontur viereckig mit schwach konvexen Seiten und abgerundeten Ecken. Poren klein, subäquatorial. Exine dünn, unter $1 \mu$ dick. Schwache Tumeszenz. Keine Anulusbildung. Kein Labrum.

Nur ein Pollenkorn wurde im Sandstein der oberen Hakobuchi-Schichtengruppe bei der Talspere von Ooyubari im Yubari-Kohlenfeld, Hokkaido gefunden.

Der Verfasser kann es nur als Polypóropollenites sp. f. beschreiben.

Botanische Zugehörigkeit: fraglich.

\section{Monoporines}

\section{Formgattung: Graminidites (CoOKsoN) R. POTONIÉ}

1947. Graminidites CooKson, S. 134.

1960. Graminidites (Cookson) ex R. PotoniE, S. 111.

Graminidites microapertus $\mathrm{n}$. sp.

Taf. 33, Fig. 25; Taf. 42, Fig. 12.

D i a g no s e : Ca. 23-24 $\mu$ groß. Figura kugelig, sekundär verfaltet. Äquator, wenn nicht verfaltet, circulär. Nur eine Pore ist vorhanden, $\pm 1.5 \mu$ im Durchmesser. Keine Anulusbildung. Keine Tumeszenz. Exine dünn, $\pm 0.5 \mu$ dick, intrapunctat bis chagrenat.

H o l o ty pus : Ca. $23 \mu$ groß; Taf. 33, Fig. 25; Präparat GK-V 3131.

Locus typicus: Hatsune-Sawa, Ooyubari, Yubari-Kohlenfeld, Hokkaido.

Stratigraphisches Verhalten: Diese Spezies fand sich bisher im Kohlenflöz der unteren Hakobuchi-Schichtengruppe (Campan) von HatsuneSawa und im Sandstein der oberen Hakobuchi-Schichtengruppe (Maastricht) bei der Talspere von Ooyubari im Yubari-Kohlenfeld, Hokkaido. Sie tritt sehr selten auf. 
Vergleich: I. C. Cookson (1947) hat die ähnlichen Pollen, Monoporites (Graminidites) media Cookson (S. 134, Taf. 15, Fig. 41-42) und Monoporites subreticulata Cookson (S, 134. Taf. 15, Fig. 43) aus den tertiären Braunkohlen und Sandsteinen des Kerguelen Archipels beschrieben. Die erstere besitzt granulate Exine und die letztere reticulate Exine.

B. L. MEYER (1956) hat die ähnlichen Formen, Monoporopollenites gramineoides MEYER (S. 111, Taf. 4, Abb. 29) und Monoporopollenites sparganioides MEYeR (S. 111, Taf. 4, Abb. 28) aus den jungtertiären Braunkohlen im östlichen Bayern (Süddeutschland) beschrieben. Dabei hat er keine Beschreibung der Formgattung Monoporopollenites gegeben. R. PoToniÉ (1960) hat die Diagnose für Monoporopollenites emendiert gegeben. Die gramineoides-Form besitzt glatte Exine und die sparganioides-Form baculate Exine.

H. Weyland und H. D. Pflug (1957) haben zwei neue Pollen mit einer Pore, Monoporopollenites solaris WeYland \& PFLUG (S. 103, Taf. 22, Fig. 36-37) und Monoporopollenites gramineus Weyland \& Pflug (S. 104, Taf. 22, Fig. 12-13) aus der pliozänen Braunkohle von Ptolemais in Nordgriechenland beschrieben. Die Exine der solaris-Form ist deutlich zweischichtig und reticulat. Die gramineus-Form besitzt Porenränder mit deutlichem Vestibulum. Ihre Exine ist deutlich zweischichtig.

R. Potonié (1960) hat Monoporopollenites sp. (S. 470, Taf. 1, Fig. 26, Abb. 8) aus der eozänen Kohle von Kalewa in Burma beschrieben. Diese Form besitzt eine Pore mit Anulus. Ihre Exine ist glatt.

Botanische Zugehörigkeit: Gramineae?

\section{Plicates}

Triptyches

\section{Formgattung: Tricolpopollenites THOMSON \& PFLUG}

1953. Tricolpopollenites Thomson \& PFLUG, S. 95.

1960. Cornaceoipollenites R. PotoniE, S. 93.

Tricolpopollenites minutiretiformis $\mathrm{n}$. sp.

Taf. 38, Fig. 4-7; Taf. 41, Fig. 16, 17.

D i a g n os e : $19.3-29 \mu$ groß. Figura spindelförmig oder kugelförmig. Polkappenkontur zugespitzt bis halbkugelig oder unterhalbkugelig. Breitenlängenindex 0.7 bis über 1 . Drei Colpi verlaufen von Pol zu Pol. Schmale \pm parallele Cavernae, die polwärts kaum konvergieren. Exine intrabaculat, 1 bis $1.5 \mu$ dick; Oberfläche der Exine fein reticulat; ihr Netztlumen unter $0.5 \mu$ (rundlich).

H ol o ty pus : Ca. $23 \mu$ groß; Taf. 38, Fig. 17; Präparat GK-V 3142.

Locus typicus: Hatsune-Sawa, Ooyubari, Yubari-Kohlenfeld, Hokkaido. 
Stratigraphisches Verhalten: Diese neue Spezies wurde bisher im Kohlenflöz der unteren Hakobuchi-Schichtengruppe (Campan) von Hatsune-Sawa und im Feinsandstein der oberen Hakobuchi-Schichtengruppe (Maastricht) bei der Talspere von Ooyubari, Yubari-Kohlenfeld, Hokkaido gefunden.

Vergleich: Morphologisch ähnliche Form, Tricolpopollenites microreticulatus TAKAHASHI (1961, S. 318, Taf. 23, Fig. 37-43) hat der Verfasser (1961) aus den miozänen und paläogenen Schichten von Westjapan beschrieben. Die microreticulatus-Form besitzt die rundlichen Netzlumen der bis $1 \mu$ Breite. Man kann die minutiretiformis Form nach Größe der Netzlumen und Skulptur der Exine von der microreticulatus-Form unterschieden werden.

Botanische Zugehörigkeit: Salix (?), Platanus (?).

Tricolpopollenites conicus $\mathrm{n}$. sp.

Taf. 39, Fig. 10, 11.

D i a g nos e : Ca. 26-27 $\mu$ groß. Figura breit-ellipsoidisch bis kugelig. Polkappenkontur zugespitzt bis unterhalbkugelig. Breitenlängenindex der Figur ist 0.74. Drei Colpi verlaufen von Pol zu Pol. Exine in lichter Anordnung mit kleinen Stacheln besetzt (Stacheln $\pm 0.5 \mu$ hoch). Exine dünn, unter $1 \mu$ dick.

H o l oty pus : Ca. $26 \mu$ groß; Taf. 39, Fig. 10; Präparat GK-V 3131.

Locus typicus: Hatsune-Sawa, Ooyubari, Yubari-Kohlenfeld, Hokkaido.

Stratigraphisches Verhalten: Diese neue Form trat bisher nur im Kohlenflöz der unteren Hakobuchi-Schichtengruppe (Campan) von Hatsune-Sawa im Yubari-Kohlenfeld, Hokkaido auf.

Vergleich: Die mitteleuropäische alttertiäre Spezies, Tricolpopollenites spinosus (R. Рот.) Thomson \& Pflug (1953, S. 98, Taf. 11, Fig. 165-172; Taf. 12, Fig. 1-4), besitzt Stacheln in dichter Anordnung. Ihre Stacheln sind ca. 2 bis $5 \mu$ lang. Deshalb kann die conicus-Form nach Größe und Anordnung der Stacheln von der spinosus-Form unterschieden werden.

Botanische Zugehörigkeit: fraglich.

\section{Tricolpopollenites umiensis TAKAHASHI \\ Taf. 39, Fig. 1.}

1957. Tricolpopollenites umiensis, K. TAKAHAShI, S. 217, Taf. 38, Fig. 37-39; Taf. 39, Fig. 31-32.

1961. Tricolpopollenites umiensis, K. Takahashi, S. 312, Taf. 22, Fig. 12-23.

Ca. $31.5 \mu$ groß. Äquatorkontur elliptisch. Figura spindelförmig. Breitenlängenindex 0.63. Struktur der Exine intrarugulat. Exine ca. $1.3 \mu$ dick. Drei schmale, \pm parallele Colpi verlaufen von Pol zu Pol. Cavernae schmal. Polkappenkontur zugespitzt.

Das vorliegende Pollenkorn ist der umiensis-Form, die vom Verfasser (1957, 
1961) aus den tertiären Schichten von Westjapan beschrieben und abgebildet wurde, gleich.

Die Art ist den mitteleuropäischen tertiären Spezies, Tricolpopollenites henrici (R. Рот.) (Pollenites henrici R. РотоNiÉ, 1931a, S. 332, Tab. 2, Fig. 19; P. W. Thomson \& H. D. Pflug, 1953, S. 95, Taf. 11, Fig. 30-42) und Tricolpopollenites microhenrici (R. Рот.) (P. W. Thomson \& H. D. PFLUG, 1953, S. 96, Taf. 11, Fig 62-110), ähnlich. Die erstere kann nach Größe, Exinenstärke und Breitenlängenindex von der henrici-Form und nach Struktur der Exine und Breitenlängenindex von der microhenrici-Form unterschieden werden. Die umiensis-Form ist auch der japanischen tertiären Spezies, Tricolpopollenites ditis Takahashi (1957, S. 218-219, Taf. 38, Fig. 46-49; Taf. 39, Fig. 39-44; 1961, S. 312, Taf. 22, Fig. 24-31), morphologisch ähnlich. Die erstere kann nach Struktur der Exine von der letzteren unterschieden werden.

Botanische Zugehörigkeit: Cupuliferae.

\section{Tricolpopollenites ef. ditis TAKAHASHI \\ Taf. 39, Fig. 2.}

1957. Tricolpopollenites ditis, K. TAKAhASHI, S. 218-219, Taf. 38, Fig. 46-49; Taf. 39, Fig. 39-44.

1961. Tricolpopollenites ditis, K. TAKahashi, S. 312, Taf. 22, Fig. 24-31.

Ca. $26 \mu$ groß. Äquatorkontur elliptisch. Figura spindelförmig. Breitenlängenindex ca. 0.7. Polkappenkontur zugespitzt. Drei Colpi verlaufen von Pol zu Pol. Cavernae schmal. Exine sehr eng vereinigt, sehr dünn, chagrenat.

Das betreffende Pollenkorn kann man von der ditis-Form, die vom Verfasser (1957, 1961) aus den tertiären Schichten von Westjapan beschieben und abgebildet wurde, fast nicht unterscheiden. Deshalb zeigt der Verfasser hier das als Tricolpopollenites cf. ditis TAKAHASHI.

Die ditis-Form ist der mitteleuropäischen tertiären Spezies Tricolpopollenites henrici (R. Рот.) (Pollenites henrici R. PotoniÉ, 1931a, S. 332, Tab. 2, Fig. 19; P. W. Thomson \& H. D. Pflug, 1953, S. 95, Taf. 11, Fig. 30-42) und der japanischen tertiären Spezies Tricolpopollenites umiensis TAKAHASHI (1957, S. 217, Taf. 38, Fig. 37-39; Taf. 39, Fig. 31-32; 1961, S. 312, Taf. 22, Fig. 12-23) morphologisch ähnlich. Die erstere kann nach Exinenstärke und -struktur von den zwei letzteren unterschieden werden.

Botanische Zugehörigkeit: Cupuliferae.

Tricolpopollenites meinohamensis TAKAHASHI subsp. rotundus TAKAHASHI Taf. 39, Fig. 3, 6 .

1961. Tricolpopollenites meinohamensis subsp. rotundus, K. TAKAHASHI, S. 315, Taf. 23, Fig. 16-18.

Ca. $20 \mu$ groß. Figura kugelig oder breit-ellipsoidisch. Breitenlängenindex ca. 0.8. Polkappenkontur etwas halbkugelig. Drei Cavernae schmal. Sie kon- 
vergieren polwärts, kommunizieren an den Polen. Exine sehr dünn, chagrenat.

Die rotundus-Form wurde im Kohlenflöz der unteren Hakobuchi-Schichtengruppe (Campan) von Hatsune-Sawa im Yubari-Kohlenfeld gefunden. Der Verfasser hat schon sie im Paläogen und Miozän von Westjapan durchläufig bemerkt. Sie ist der japanischen tertiären Spezies Tricolpopollenites chikushiensis Takahashi subsp. globulosus Takahashi (1961, S. 316-317, Taf. 23, Fig. 26-31) ähnlich. Die Exine der globulosus-Form ist intrabaculat bis intrarugulat. Deshalb kann man die rotundus-Form nach Exinenstruktur von der globulosusForm unterscheiden.

Botanische Zugehörigkeit: fraglich.

\section{Tricolpopollenites ef. microreticulatus TAKAHASHI \\ Taf. 39, Fig. 4.}

1961. Tricolpopollenites microreticulatus, K. TAKAhaShI, S. 318, Taf. 23, Fig. 37-43.

Ca. $21 \mu$ groß. Figura kugelig. Breitenlängenindex ca. 0.85. Polkappenkontur halbkugelig bis unterhalbkugelig. Drei schmale Cavernae konvergieren polwärts. Exine sehr dünn, fein reticulat; Netzlumen $\pm 1 \mu$ breit.

Die Figur 4 kann der Verfasser hier als Tricolpopollenites cf. microreticulatus Takahashi bezeichnen. Sie fand sich im Kohlenflöz der unteren HakobuchiSchichten (Campan) von Hatsune-Sawa, Ooyubari, Hokkaido.

Diese Spezies wurde bisher im Paläogen und Miozän von West- und Nordostjapan und durchläufig gefunden.

Botanische Zugehörigkeit: Salix, Platanus u. a. (?).

\section{Tricolpopollenites cf. subasper TAKAHASHI \\ Taf. 39, Fig. 7.}

1957. Tricolpopollenites subasper, K. TAKAHASHI, S. 217-218, Taf. 38, Fig. 42-43.

1961. Tricolpopollenites subasper, K. TAKAHASHI, S. 314, Taf. 23, Fig. 12-14.

Ca. $21 \mu$ groß. Figura kugelig, nach der Polachse etwas gestreckt. Breitenlängenindex 0.8. Exine chagrenat, $1 \mu$ dick. Drei schmale Colpi verlaufen fast parallel polwärts. Polkappenkontur unterhalbkugelig.

Die hier bestehende Figur ist der tiefpaläogenen Art von Westjapan, Tricolpopollenites subasper TAKAHASHI (1957, S. 217-218, Taf. 38, Fig. 42-43), fast identisch, aber der Verfasser kann sie hier als Tricolpopollenites cf. subasper TAKaHashi nennen. Sie wurde nur im Kohlenflöz der unteren HakobuchiSchichtengruppe von Hatsune-Sawa, Ooyubari, Hokkaido wenig gefunden. Sie wurde bisher vom Verfasser für eine eozäne Form von Westjapan angesehen.

Botanische Zugehörigketi: Vielleicht Cupuliferae.

\section{Tricolpopollenites sp. a.}

Taf. 39, Fig. 5a, b.

Ca. 18-21.5 $\mu$ groß. (Fig. 5: $18 \mu$ groß). Figura kugelig. Breitenlängen- 
index ca. 0.8. Polhemisphäre halbkugelig. Drei gleichmäßig schmale Colpi, die polwärts mehr oder weniger konvergieren. Exine dünn, bis $\pm 1 \mu$ dick, fein verrucat. Warzen $\pm 1 \mu$ hoch. Größere Warzen ordnen sich besonders zwischen den Colpen.

Nur ein Pollenkorn wurde im Kohlenflöz der unteren Hakobuchi-Schichtengruppe von Hatsune-Sawa, Ooyubari, Hokkaido gefunden.

Botanische Zugehörigkeit: fraglich.

Tricolpopollenites sp. b.

Taf. 39, Fig. 8.

$27.5 \mu$ groß. Figura spindelförmig oder ellipsoidisch. Breitenlängenindex ca. 0.7. Polkappenkontur zugespitzt. Drei Colpi konvergieren polwärts und erreichen die Pole. Cavernae schmal. Exine dünn, $0.7 \mu$ dick, chagrenat.

Diese Form fand sich bisher nur im Kohlenflöz der unteren HakobuchiSchichtengruppe von Hatsune-Sawa, Ooyubari, Hokkaido.

Botanische Zugehörigkeit: fraglich.

Tricolpopollenites sp. c.

Taf. 39, Fig. 9.

Ca. $21 \mu$ groß. Figura spindelförmig bis ellipsoidisch. Breitenlängenindex ca. 0.5. Polhemisphäre zugespitzt. Drei schmale Colpi konvergieren polwärts. Exine $1 \mu$ dick, chagrenat.

Diese Form wurde bisher im Kohlenflöz der unteren Hakobuchi-Schichten von Hatsune-Sawa, Ooyubari, Hokkaido gefunden. Sie ist der japanischen tertiären Spezies, Tricolpopollenites vulgaris TAKAHASHI (1957, S. 218, Taf. 38, Fig. 44-45; Taf. 39, Fig. 38; 1961, S. 312, Taf. 22, Fig. 32-37), ähnlich, aber der Verfasser kann sie nur als Tricolpopollenites sp. c. zeigen.

Botanische Zugehörigkeit: Cupliferae?

\section{Tricolpopollenites sp. d.}

Taf. 39, Fig. 12.

Ca. $38.5 \mu$ groß. Figura breit-ellipsoidisch. Breitenlängenindex ca. 0.75 . Polhemisphäre unterhalbkugelig bis halbkugelig. Drei schmale Colpi konvergieren polwärts. Cavernae sehr schmal. Exine dünn, chagrenat.

Die vorlieegnde Art wurde bisher nur im Kohlenflöz der unteren HakobuchiSchichtengruppe von Hatsune-Sawa, Ooyubari, Hokkaido gefunden.

Botanische Zugehörigkeit: fraglich.

\section{Tricolpopollenites sp. e. \\ Taf. 41, Fig. 18.}

Ca. $16.5 \mu$ groß. Figura kugelig. Breitenlängenindex ca. 0.85 . Drei schmale und kurze Colpi verlaufen \pm parallel. Exine dünn, $1 \mu$ dick, chagrenat bis schwach glatt. 
Diese Form wurde bisher im Sandstein der oberen Hakobuchi-Schichtengruppe (Maastricht) bei der Talspere von Ooyubari, Hokkaido gefunden.

Botanische Zugehörigkeit: fraglich.

\section{Tricolpopollenites sp. $\mathrm{f}$.}

Taf. 41, Fig. 19.

$20 \mu$ lang, $12.6 \mu$ breit. Figura spindelförmig. Breitenlängenindex ca. 0.6. Polhemisphäre zugespitzt. Drei schmale Colpi konvergieren polwärts. Exine dünn, schwach striat.

Die betreffende Spezies fand sich im Sandstein der oberen HakobuchiSchichtengruppe (Maastricht) bei der Talspere von Ooyubari, Hokkaido. Sie ist der japanischen Art, Tricolpopollenites striatellus TAKAHASHI (1961, S. 319, Taf. 23, Fig. 50-51), ähnlich, aber der Verfasser kann sie hier nur als Tricolpopollenites sp. f. zeigen.

Botanische Zugehörigkeit: fraglich.

\section{Tricolpopollenites sp. g. \\ Taf. 39, Fig. 13.}

$19 \mu(\times 12.7 \mu)$ groß. Kontur elliptisch. Figura spindelförmig. Breitenlängenindex ca. 0.67. Polhemisphäre zugespitzt. Drei schmale Colpi konvergieren polwärts. Exine dünn, fein striat.

Diese Form fand sich bisher im Kohlenflöz der unteren Hakobuchi-Schichtengruppe von Hatsune-Sawa, Ooyubari, Hokkaido.

Botanische Zugehörigkeit: fraglich.

\section{Tricolpopollenites sp. $\mathrm{h}$. \\ Taf. 39, Fig. 14.}

Ca. $23 \mu$ groß. Kontur elliptisch. Figura ellipsoidisch. Breitenlängenindex ca. 0.76. Drei schmale Colpi konvergieren polwärts. Exine punctat; Punkte ordnen sich striat.

Diese Form wurde nur im Kohlenflöz der unteren Hakobuchi-Schichtengruppe von Hatsune-Sawa, Ooyubari, Hokkaido gefunden.

Botanische Zugehörigkeit: fraglich.

\section{Tricolpopollenites sp. i.}

Taf. 39, Fig. 15.

Ca. 20.5 $\mu$ groß. Kontur etwas rundlich. Figura etwas kugelig. Breitenlängenindex ca. 0.8. Polhemisphäre unterhalbkugelig. Drei Colpi schmal. Exine fein punctat.

Nur ein Pollenkorn wurde bisher im Kohlenflöz der unteren HakobuchiSchichtengruppe von Hatsune-Sawa, Ooyubari, Hokkaido gefunden. 
Botanische Zugehörigkeit: fraglich.

\section{Formgattung: Rhoipites WoDEHouse}

1933. Rhoipites WodeHouse, S. 513.

1960. Rhoipites WodeHouse in R. PotoniE, S. 100-101.

Rhoipites ? minutireticulatus n. sp.

Taf. 39, Fig. 21-26; Taf. 41, Fig. 21.

D i a g nos e : Ca. 20.5-26.5 $\mu$ groß. Kontur kreisförmig bis elliptisch. Figura kugelig bis ellipsoidisch. Breitenlängenindex 0.7 bis 0.9. Polhemisphäre halbkugelig bis zugespitzt. Drei schmale Colpi konvergieren polwärts. Cavernae schmal. Alle Poren rundlich, seitlich über die Cavernae hinausgreifend. Exine verhältnismäßig dick ( 1 bis $2 \mu$ dick), intrabaculat. Oberfläche der Exine fein reticulat; Netzlumen unter $0.5 \mu$.

H o l o ty p u s : Ca. $26 \mu$ groß; Taf. 39, Fig. 26 ; Präparat GK-V 3143.

Locus typicus: Hatsune-Sawa, Ooyubari, Yubari-Kohlenfeld, Hokkaido.

Stratigraphisches Verhalten: Diese Art wurde in der unteren und oberen Hakobuchi-Schichtengruppe von Ooyubari im Yubari-Kohlenfeld gefunden.

Vergle i ch: Ob diese Form zu Rhoipites oder zu Tricolporopollenites gehört, ist noch nicht klar, aber der Verfasser nimmt vorläufig Rhoipites auf.

Die betreffende Form ist der Art, Tricolpopollenites minutiretiformis TAKAHASHI (in dieser Arbeit, S. 238-239, Taf. 39, Fig. 4-27; Taf. 41, Fig. 16, 17), morphologisch ähnlich, aber die letztere besitzt keine Pore.

Die minutireticulatus-Form ist der mitteleuropäischen tertiären Spezies, Tricolporopollenites microreticulatus THomson \& Pflug (1953, S. 106, Taf. 14, Fig. 27-42), ähnlich. Die erstere kann nach Skulptur der Exine und nach gegenseitiges Verhältnis der Poren und Cavernae von der letzteren unterschieden werden.

Botanische Zugehörigkeit: fraglich.

\section{Formgattung: Tricolporopollenites THOMSON \& PFLUG}

1953. Tricolporopollenites Thомson \& PfLuG, S. 98.

Tricolporopollenites minor TAKAHASHI

Taf. 39, Fig. 16-18; Taf. 41, Fig. 22-25; Taf. 42, Fig. 10.

1961. Tricolporopollenites minor, K. TAKahashI, S. 320, Taf. 24, Fig. 18-31.

Kontur rundlich. Figura kugelig bis breit-ellipsoidisch. Breitenlängenindex ca. 0.8-1. Polhemisphäre halbkugelig. Drei Colpi konvergieren polwärts. Schmale Cavernae erreichen die Pole. Poren seitlich über die Cavernae hinaus- 
greifend. Kurze Ruge. Exine dünn, unter $1 \mu$ dick, schwach glatt bis chagrenat.

Diese Form wurde bisher im Alttertiär und Miozän von Westjapan häufig gefunden. Sie fand sich hier im Kohlenflöz der unteren Hakobuchi-Schichtengruppe von Hatsune-Sawa und in den Sandsteinen der oberen Hakobuchi-Schichten bei der Talspere von Ooyubari, Hokkaido.

Die Figur 25 der Tafel 19 gehört zu dieser Spezies sehr zweifelhaft.

Botanische Zugehörigkeit: Vielleicht Cyrillaceae.

\section{Tricolporopollenites sp. a. \\ Taf. 39, Fig. 19.}

Diese Spezies fand sich bisher nur im Kohlenflöz der unteren HakobuchiSchichten von Hatsune-Sawa, Ooyubari, Hokkaido.

Ca. $26.8 \mu$ groß. Kontur elliptisch. Figura spindelförmig. Breitenlängenindex ca. 0.65. Drei Colpi konvergieren polwärts. Cavernae schmal. Poren rundlich, seitlich über die Cavernae hinausgreifend. Exine dünn, fein striat.

Botanische Zugehörigkeit: fraglich.

\section{Tricolporopollenites sp. b.}

Taf. 39, Fig. 20.

$26.5 \mu(\times 21.3 \mu)$ groß. Kontur breit elliptisch. Figura breit ellipsoidisch. Breitenlängenindex ca. 0.8. Drei schmale Colpi konvergieren polwärts. Poren verhältnismäßig groß, etwas meridional gestreckt, seitlich über die Cavernae hinausgreifend. Exine dünn, $0.9 \mu$ dick, fein intrarugulat.

Diese Form wurde bisher nur im Kohlenflöz der unteren HakobuchiSchichten von Hatsune-Sawa, Ooyubari, Hokkaido gefunden.

Botanische Zugehörigkeit: fraglich.

\section{Tricolporopollenites sp. c.}

Taf. 39, Fig. 27.

$30.5 \mu(\times 21.8 \mu)$ groß. Kontur elliptisch. Figura ellipsoidisch. Polhemisphäre etwas zugespitzt. Breitenlängenindex ca. 0.7. Drei Colpi konvergieren polwärts. Cavernae schmal. Porus rundlich, seitlich über die Cavernae hinausgreifend. Exine $1.4 \mu$ dick, fein intrabaculat.

Die vorliegende Art trat bisher nur im Kohlenflöz der unteren HakobuchiSchichten von Hatsune-Sawa, Ooyubari, Hokkaido auf.

Botanische Zugehörigkeit: fraglich.

\section{Tricolporopollenites sp. d.}

Taf. 39, Fig. 29.

Ca. $34.5 \mu$ groß. Kontur rundlich. Figura kugelig. Polhemisphäre halbkugelig. Breitenlängenindex ca. 0.85 . Drei Colpi konvergieren polwärts. Ca- 
vernae verhältnismäßig schmal. Porus klein, rundlich, von der Caverna umschrieben. Exine dünn, fein rugulat.

Diese Form wurde bisher nur im Kohlenflöz der unteren Hakobuchi-Schichtengruppe von Hatsune-Sawa, Ooyubari, Hokkaido gefunden.

Botanische Zugehörigkeit: fraglich.

Ptychopolyporines

Formgattung: Tetracolporopollenites THOMsON \& PFLUG

1953. Tetracolporopollenites Thomson \& PfLUG, S. 108.

Tetracolporopollenites cretaceus $n$. sp.

Fig. 39, Fig. 30-33.

D i a g n o s e : Tetracolporat. Ca. $37.5-47.5 \mu$ groß. Kontur rundlich bis breit elliptisch. Figura eiförmig bis breit ellipsoidisch. Polhemisphäre unterhalbkugelig bis halbkugelig. Breitenlängenindex 0.76-0.9. Vier Colpi verlaufen fast parallel und konvergieren schwach polwärts. Schmale Caverane erreichen nicht den Pol. Porus groß, rundlich, seitlich über die Cavernae hinausgreifend. Exine 1 bis $2 \mu$ dick, chagrenat.

Die Figur 30 gehört zu dieser Spezies sehr zweifelhaft, da sie nur die kleinen und von den Cavernae umschriebenen Poren besitzt.

H o l o t y p u s : $40 \mu$ groß; Taf. 39, Fig. 33a, b; Präparat GK-V 3152.

Locus typicus: Hatsune-Sawa, Ooyubari, Yubari-Kohlenfeld, Hokkaido.

Stratigraphisches Verhalten: Diese Form wurde bisher nur im Kohlenflöz der unteren Hakobuchi-Schichtengruppe von Hatsune-Sawa, Ooyubari, Hokkaido gefunden.

Verglei ch: Die betreffende Form ist einigen Pollen, die von S. Sato (1961) aus den Osoushinai-Schichten der Hakobuchi-Schichtengruppe als unbestimmbare Pollen (Sapotaceae?) abgebildet wurden, morphologisch ähnlich, aber er hat keine Beschriebung dieser Pollen gegeben (S. SATo, 1961, Taf. 1, Fig. 10, 11, 12a, b, 15). Deshalb kann der Verfasser die vorliegende Form mit den Pollen SATo's nicht vergleichen.

Die mitteleuropäische alt- und mitteltertiäre Spezies Tetracolporopollenites sapotoides Thomson \& Pflug (1953, S. 110, Taf. 15, Fig. 6-12), kann man mit der cretaceus-Form vergleichen. Die japanische Form kann nach Form der Cavernae und Struktur der Exine von der sapotoides-Form unterschieden werden.

Botanische Zugehörigkeit: Sapotaceae.

\section{Monocolpates}

Formgattung: Weylandispollis n. gen.

Di a g nose: Ein Colpus. Figura kugelig bis ellipsoidisch. Äquatorkontur rundlich bis elliptisch. Polkappenkontur unterhalbkugelig bis zugespitzt. 
Colpus den Umriß erreichend, dort beiderseits erweitert, in der Mitte enger werdend. Exine reticulat.

Genotypus: Weylandipollis retiformis n. sp.

Weylanripollis retiformis $\mathrm{n}$. $\mathrm{sp}$.

Taf. 32, Fig. 1-17.

D i a $\mathrm{g}$ n o s e : $36-46 \mu$ groß. Figura kugelig bis ellipsoidisch. Umrißlinie rundlich bis ellipsoidisch. Reticulate Skulptur. Netzlumen 1 bis $6 \mu$ breit. Colpus den Umriß erreichend, dort beiderseits erwaitert, in der Mitte eng oder wenig erweitert. Colpus immer von Randwulst umrahmt. Netz entlang den Colpenwulst kleiner ( $\pm 1 \mu$ breit).

H o loty pu s : $40 \mu$ groß; Taf. 32, Fig. 2a, b; Präparat GK-V 3143.

Locus typicuus: Hatsune-Sawa, Ooyubari, Yubari-Kohlenfeld, Hokkaido.

Stratigraphisches Verhalten: Diese neue Form wurde bisher nur im Kohlenflöz der unteren Hakobuchi-Schichtengruppe (Campan) von Hatsune-Sawa, Yubari-Kohlenfeld (Hokkaido) gefunden.

Vergleich: Die vorliegende Form ist mit der Form der Gattung Liliacidites COUPER (1953) morphologisch näher verwandtschaftlich: Liliacidites kaitangataensis Couper (1953, S. 56, Taf. 7, Fig. 97) aus den oberkretazeischen Schichten im Kaitangata-Kohlenfeld, Neuseeland, Liliacidites variegatus CoUPER (1953, S. 56, Taf. 7, Fig. 98-99) aus den oligozänen Schichten von dem MatauraTal, Südland (Neuseeland) und Liliacidites intermedius CoUPER (1953, S. 56, Taf. 7, Fig. 100) aus den oberkretazeischen Schichten im Morley Gebiet, Neuseeland. Die Gattung Weylandipoliis kann hauptsächlich nach Form des Pollens und der Colpen von der Gattung Liliacidites unterschieden werden.

Botanische Zugehörigkeit: Liliaceae, Araceae u. a. kommen in Frage.

Formgattung: Monocolpopollenites Thomson \& PFLUG

1953. Monocolpopollenites Thomson \& Pflug, S. 62.

1958. Palmaepollenites R. Potoní, S. 97-98.

Wie S. MANum (1962, S. 47) schon geschrieben hat, war die erste gültige Beschreibung von Palmaepollenites in R. PотоNiÉ (1958, S. 97). Die Gattung Monocolpopollenites wurde schon 1953 von THOMson und PFLUG gültig beschrieben. Deshalb hat Monocolpopollenites die Priorität.

\section{Monocolpopollenites shiynparoensis n. sp. \\ Taf. 33, Fig. 1-5.}

D i a g nos e : Ca. 30-42 $\mu$ groß. Figura linsenförmig. Der Äquator hat eine elliptische Kontur. Polkappenkontur zugespitzt bis halbkugelig. Reticulate Skulptur, polyeckig. Netzlumen unter 5 bis $6 \mu$ breit. Muri sind nicht immer vorhanden, mit schwach warzenförmigem Querschnitt. Colpus schmal, bogig 
verlaufend. Colpus klafft selten.

H o l o ty pu s: $40 \mu$ groß; Taf. 33, Fig. 2; Präparat GK-V 3143.

Locus typicus: Hatsune-Sawa, Ooyubari, Yubari-Kohlenfeld, Hokkaido.

Stratigraphisches Verhalten: Diese Spezies wurde im Kohlenflöz der unteren Hakobuchi-Schichtengruppe (Campan) von Hatsune-Sawa, Yubari-Kohlenfeld (Hokkaido) gefunden.

Vergle i ch: Die betreffende Spezies ist den mitteleuropäischen paläogenen Formen, Monocolpopollenites observatus PfLUG (P. W. Thomson \& H. D. PFlug, 1953, S. 62, Taf. 4, Fig. 17) und Monocolpopoilenites areolatus (R. Pot.) Thomson \& Pflug subsp. retareolatus Pflug (P. W. Thomson \& H. D. Pflug, 1953, S. 63, Taf. 4, Fig. 50-60), morphologisch ähnlich. Die shiyuparoensisForm ist kleiner als die observatus-Form und das Netz der ersteren Exine ist gröber als das der letzteren. Die Exine der retareolatus-Form ist vielmehr rugulat bis gemmat.

R. A. COUPER (1953) hat morphologisch ähnliche Formen, Liliacidites variegatus CouPER (S. 56, Taf. 7, Fig. 98-99) aus den oligozänen Schichten im Mataura-Tal, Südland, Neuseeland, Liliacidites intermedius CouPER (S. 56, Taf. 7, Fig. 100) aus den oberkretazeischen Schichten im Morley Gebiet, Neuseeland und Liliacidites kaitangataensis CouPER (S. 56, Taf. 7, Fig. 97) aus den oberkretazeischen Schichten im Kaitangata-Kohlenfeld, Neuseeland beschrieben. Das Netz der shiyuparoensis-Form ist gröber als das der variegatus-Form. Die intermedius-Form ist etwas gröber als die japanische Form und das Netz der ersteren ist etwas kleiner als das der letzteren. Die kaitangataensis-Form ist viel größer als die japanische Form.

Botanische Zugehörigkeit: fraglich.

\section{Monocolpopollenites kyushuensis TAKAHASHI \\ Taf. 33, Fig. 6-8; Taf. 41, Fig. 13, 14.}

1961. Monocolpopollenites kyushuensis, K. TAKaHASHI, S. 292, Taf. 16, Fig. 17-23.

Die monocolpate kyushuensis-Form wurde 1961 vom Verfasser aus dem Paläogen und Miozän Westjapans erstmals beschrieben.

Die betreffenden Figuren wurden aus den oberen und unteren HakobuchiSchichtengruppe (Campan-Maastricht) von Ooyubari, Yubari-Kohlenfeld (Hokkaido) gefunden.

Sie sind der mitteleuropäischen tertiären Form, Monocolpopollenites tranquillus (R. Рот.) Thomson \& Pflug (Pollenites tranquillus R. Рот., 1934, S. 51, Tab. 2, Fig. 3, 8; P. W. Thomson \& H. D. Pflug, 1953, S. 62-63, Taf. 4, Fig. 24-37, 39-47) ähnlich, aber die erstere hat meistens linsenförmige Äquatorkontur und Figura und die letztere hat meistens vier- bis sechseckige Kontur und tafelförmige Figur.

Botanische Zugehörigkeit: Neben Palmae wäre am ehesten an Ginkgoinae zu denken. 


\section{Monocolpopollenites pfugii TAKAHASHI}

Taf. 33, Fig. 12-15.

1961. Monocolpopollenites pfugii, K. TAKahashi, S. 294, Taf. 16, Fig. 31-32.

Monocolpopollenites pflugii TAKAHASHI wurde 1961 vom Verfasser aus dem Paläogen Westjapans erstmals beschrieben.

Die betreffenden Figuren wurden aus dem Kohlenflöz der unteren HakobuchiSchichtengruppe (Campan) von Hatsune-Sawa, Yubari-Kohlenfeld (Hokkaido) abgebildet.

Sie sind Liliacidites variegatus Couper (1953, S. 56, Taf. 7, Fig. 98-99) aus den oligozänen Schichten im Mataura-Tal, Südland (Neuseeland) sehr ähnlich. Die letztere besitzt feineres Netz (meistens $1 \mu$ breit) als die erstere.

Botanische Zugehörigkeit: Palmae?

Monocolpopollenites asymmetricus n. sp.

Taf. 33, Fig. 10.

1961. Monocolpopollenites kyushuensis, K. TaKahashi, Taf. 16, Fig. 22.

D i a g n o s e : Ca. $23-29 \mu$ groß. Äquatorkontur breit-linsenförmig oder viereckig mit verhältnismäßig gespitzten Ecken. Zwei Ecken liegen an den Schnittpunkten mit der Colpus-Ebene. Colpus nicht symmetrisch, meistens mehr oder weniger bogig, immer wulstig. Colpus klafft nie. Exine dünn, stets chagrenat. Der Halbkreis, der von dem ausgebogenen Colpus geteilt wurde, ist mehr dunkel als der andere sichtbar.

H ol ot y p u s : Ca. $26 \mu$ lang, $17 \mu$ breit; Taf. 33, Fig. 10; Präparat GK-V 3171.

Locus typicus: Hatsune-Sawa, Ooyubari, Yubari-Kohlenfeld, Hokkaido.

Stratigraphisches Verhalten: Diese Form wurde bisher in der unteren Hakobuchi-Schichtengruppe (Campan) von Hatsune-Sawa, YubariKohlenfeld (Hokkaido) und den tertiären Schichten von Westjapan gefunden.

Vergle i ch: Bisher wurde diese Form als Monocolpopollenites kyushuensis TAKAHASHi umfasst, aber der Verfasser hat sie als die neue Spezies Monocolpopollenites asymmetricus beschrieben. Die asymmetricus-Form ist Monocolpopollenites kyushuensis TAKAHASHI (1961, S. 292, Taf. 16, Fig. 17-21, 23) sehr ähnlich, aber die erstere kann nach Eigentümlichkeiten der Exine und Colpen von der letzteren morphologisch unterschieden werden.

Botanische Zugehörigkeit: Cycadaceae oder Ginkgoaceae kommen in Frage.

\section{Monocolpopollenites sp. a}

Taf. 33, Fig. 9.

Monocolpater Pollen. Ca. $11 \mu$ lang und $6.3 \mu$ breit. Äquatorkontur elliptisch. Exine dünn, schwach chagrenat. Colpus geradlinig verlaufend. 
Dieser Pollen wurde bisher nur im Kohlenflöz der unteren Hakobuchi-Schichtengruppe (Campan) von Hatsune-Sawa, Yubari-Kohlenfeld (Hokkaido) gefunden.

Botanische Zugehörigkeit: Palmae?

\section{Monocolpopollenites sp. $\mathrm{b}$}

Taf. 33, Fig. 11.

Monocolpater Pollen. $33.7 \mu$ lang und $27.5 \mu$ breit. Äquatorkontur breitelliptisch. Polkappenkontur halbkugelig. Exine dünn, chagrenat. Colpus schmal, bogig.

Diese große Form wurde bisher nur im Kohlenflöz der unteren HakobuchiSchichtengruppe (Campan) von Hatsune-Sawa, Yubari-Kohlenfeld (Hokkaido) gefunden.

Botanische Zugehörigkeit: Palmae?

Incertae Sedis

Formgattung: Aquilapollenites (Rouse) FunkHoUser

1957. Aquilapollenites Rouse, S. 370-371.

1961. Aquilapollenites (ROUSE) FunkHOUSER, S. 193-194.

\section{Aquilapollenites triauritus n. sp.}

Taf. 37, Fig. 1a-c, 2a-c.

D i a g n o s e : Isopolar. Längster Durchmesser des Körpers (der Polachse) 38.5-40 $\mu$. Figura des Körpers ellipsoidisch. Kontur des Hauptkörpers elliptisch. Drei ohrenförmige Vorsprünge mit etwas abgerundeter Spitze befinden sich je entsprechend zwischen zwei meridionalen Halbcolpi. Polachse ist eine dreizählige Symmetrieachse. Sechs Halbcolpi linienförmig oder lang-elliptisch aufgespalten, etwa $14.5 \mu$ lang. Ohrenförmige Vorsprünge 11.5 bis $12.5 \mu$ lang. Exine bis $2 \mu$ dick, fein reticulat. Netzlumen $\pm 1 \mu$ breit.

H o l o ty p u s : $38.5 \mu$ groß; Taf. 37, Fig. 2a-c; Präparat GK-V 3132.

Locus typicus: Hatsune-Sawa, Ooyubari, Yubari-Kohlenfeld, Hokkaido.

Stratigraphisches Verhalten: Diese neue Spezies fand sich bisher nur im Kohlenflöz der unteren Hakobuchi-Schichtengruppe (Campan) von Hatsune-Sawa, Yubari-Kohlenfeld, Hokkaido. Die Gattung Aquilapollenites wurde von G. E. Rouse (1957) für die Pollenkörner aus den campanischen Schichten von Westkanada neu vorgeschlagen und später von J. W. FUNKHOUSER (1961) emendiert. Aquilapollenites scheint ein charakteristischer Pollen der Oberkreide und des tiefen Paläogens in den pazifischen Randgebieten zu sein.

Vergleich: Die vorliegende Spezies ist Aquilapollenites trialatus Rouse (1957, S. 371, Taf. 2, Fig. 14, 15) aus den oberkretazeischen OldmanSchichten von Südalberta (Westkanada) ähnlich. Die erstere kann nach Form 

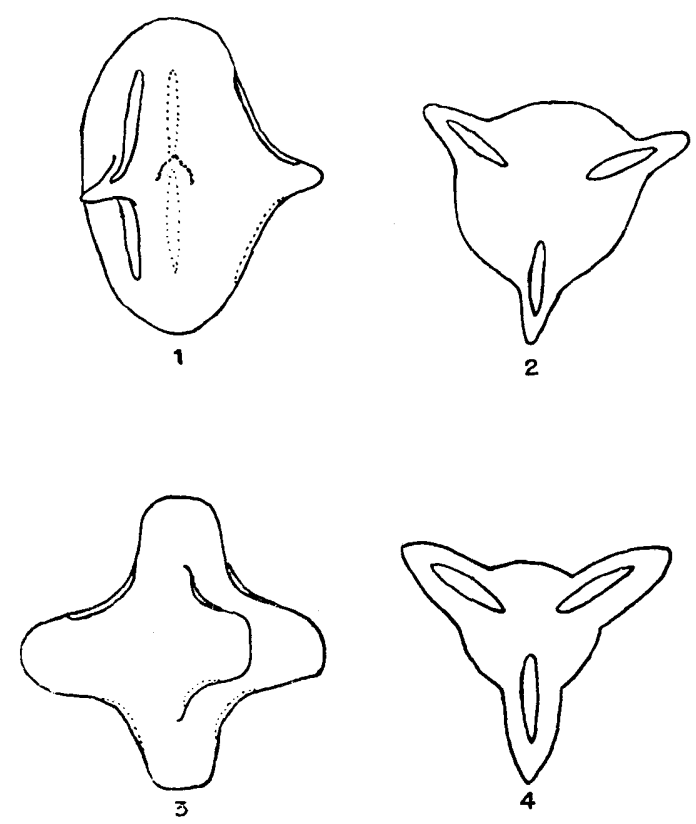

Abb. 12. Schematische Form von Aquilapollenites und die Anordnung der Colpi.

1 \& 2: Aquilapollenites triauritus- und Aquilapollenites bortalis-Form 1: Äquatoransicht, 2: Polansicht.

3 \& 4: Aquilapollenites matsumoloi- und Aquilapollenites quadrinusForm

3: ̈̈quatoransicht, 4: Polansicht.

des Körpers und der Skulptur der Exine von der letzteren unterschieden werden.

S. SAto (1961) hat Aquilapollenites hakobuchiensis SAto (S. 91-92, Taf. 1, Fig. 13-14), Aquilapollenites sp. (S. 92, Taf. 1, Fig. 20) und Aquilapollenites quadrilobus Rouse (Taf. 1, Fig. 19) aus den oberkretazeischen Schichten in Hokkaido beschrieben und abgebildet.

Die morphologisch ähnliche Form Aquilapollenites novacolpites FunkHousER (1961, S. 196, Taf. 2, Fig. 2-3) hat J. W. Funkhouser aus den Mesa Verde Schichten (Campan) von Rawlins, Wyoming (USA) beschrieben. Die novacolpites-Form besitzt drei paarige Halbcolpi und drei äquatoriale Rinnen.

E. A. Stanley (1961) hat morphologisch sehr ähnliche Spezies, Aquilapollenites reticulatus STANLEY (S. 348-349, Taf. 8, Fig. 1-12), aus den maastrichtischen Hell Creek Schichten von Crow Butte, South Dakota, USA beschrieben. Die reticulatus-Form besitzt dünnere Exine und hohe Muri.

Botanische Zugehörigkeit: fraglich.

Aquilapollenites borealis $\mathrm{n}$. sp.

Taf. 37, Fig. 3a-d, 4a-b.

D i a g nos e : Isopolar. 28.5-33 $\mu$ groß. Figura des Hauptkörpers kuge- 
lig. Kontur des Hauptkörpers kreisförmig. Drei gespitzte und ohrenförmige Vorsprünge befinden sich entsprechend in der Mitte der ein paarigen meridionalen Halbcolpi. Drei paarige Halbcolpi linienförmig oder lang-elliptisch, 6-10 $\mu$ lang. Vorsprünge dreieckig, $\pm 10 \mu$ lang. Exine 2.5 bis $3 \mu$ dick, fein punctat, wie Fingerabdrücke angeordnet.

H o l o ty pu s : Ca. $33 \mu$ groß; Taf. 37, Fig. 3a-d; Präparat GK-V 3131.

Locus typicus: Hatsune-Sawa, Ooyubari, Yubari-Kohlenfeld, Hokkaido.

Stratigraphisches Verhalten: Diese neue Spezies wurde bisher nur im Kohlenflöz der unteren Hakobuchi-Schichtengruppe (Campan) von Hatsune-Sawa, Yubari-Kohlenfeld, Hokkaido gefunden.

Vergleich: Die borealis-Form ist Aquilapollenites triauritus TAKAHASHI (in dieser Arbeit, Taf. 37, Fig. 1a-c, 2a-c) morphologisch ähnlich, aber die erstere kann nach Form des Körpers und Skulptur der Exine von der letzteren unterschieden werden.

Die vorliegende Form ist auch Aquilapollenites trialatus Rouse (1957, S. 371, Taf. 2, Fig. 14-15) aus den oberkretazeischen Oldman-Schichten von Südalberta, Westkanada ähnlich, aber die erstere kann nach Form und Größe des Hauptkörpers von der letzteren unterschieden werden.

Botanische Zugehörigkeit: fraglich.

Aquilapollenites matsumotoi n. sp.

Taf. 38, Fig. 1a-b, 3a-b.

Diagnose: Isopolar. Polachse $46-50 \mu$ lang, dreizählige Symmetrieachse. Drei subrechteckige, flügelförmige Vorsprünge 15 bis $20 \mu$ lang. Zwischengebiete der Pole und der Spitzen der äquatorialen Vorsprünge tief konkav; dort drei paarige Halbcolpi vorhanden. Sechs Halbcolpi linienförmig oder lang-elliptisch aufgespalten, etwa 11 bis $13 \mu$ lang. Von den Spitzen der Vorsprünge bis zu der Polachse 30 bis $31 \mu$ lang. Drei Vorsprünge besitzen grob-papillate Skulptur. Ihre papillate Warzen ordnen sich an den Spitzen der Flügel und in der der Seitenlinie parallel verlaufenden Mittelzone. Bei den flügelförmigen Vorsprüngen der Pole breiten sich feinere oder etwas gespitzte Warzen unregelmäßig aus. Sonstige Skulptur der Exine fein reticulat.

H oloty pus: Polachse $50 \mu$ lang (56 $\mu$ groß) ; Taf. 38, Fig. 1a, b; Präparat GK-V 3181.

Locus typicus: Hatsune-Sawa, Ooyubari, Yubari-Kohlenfeld, Hokkaido.

Stratigraphisches Verhalten: Diese neue Form wurde bisher nur im Kohlenflöz der unteren Hakobuchi-Schichtengruppe (Campan) von Hatsune-Sawa, Yubari-Kohlenfeld, Hokkaido gefunden.

Vergleich: Die vorliegende Spezies ist der kanadischen oberkretazeischen Spezies, Aquilapollenites quadrilobus Rouse (1957, S. 371, Taf. 2, Fig. 8-9), morphologisch ähnlich. Die matsumotoi-Form kann nach dem Ornament 
der Exine von der letzteren unterschieden werden.

Die matsumotoi-Form ist der amerikanischen Spezies, Aquilapollenites attenuatus Funkhouser (1961, S. 195-196, Taf. 2, Fig. 1a-c), aus den LanceSchichten (Maastricht) von Rawlins, Wyoming sehr ähnlich. Die erstere kann nur nach der feinen Skulptur von der letzteren unterschieden werden. Die Exine der ersteren ist fein reticulat.

Die matsumtoi- und attenuatus-Form ist der Spezies Aquilapollenites amplus StANLEY (1961, S. 342-346, Taf. 2, Fig. 1-4; Taf. 3, Fig. 1-5) aus den maastrichtischen Hell Creek Schichten von Crow Butte, South Dakota, USA nahestehend. Die matsumotoi-Form kann nach Anordnung und Zahl der papillaten Warzen von der amplus-Form unterschieden werden.

Botanische Zugehörigkeit: fraglich.

\section{Aquilapollenites quadrinus n. sp.}

Taf. 41, Fig. 31a-c.

D i a g n os e : Isopolar. Polachse $47 \mu$ lung, dreizählige Symmetrieachse. Drei flügelförmige Vorsprünge ca. $19 \mu$ lang, ca. $13.5 \mu$ breit, nach der Spitze allmählich schmal werdend und ihre Spitze abgerundet. Von der Spitzen der Vorsprünge bis zu der Polachse ca. $27.5 \mu$ lang. Zwischengebiete der Pole und der Spitzen der äquatorialen Vorsprünge tief konkav; dort paarige Halbcolpi vorhanden. Halbcolpi linienförmig, ca. $16 \mu$ lang. Striate Linien der Exinenskulptur verlaufen nach den Polen.

$\mathrm{H}$ o l oty pus : Polachse $47 \mu$ lang (ca. $57.5 \mu$ groß); Taf. 41, Fig. 31a-c; Präparat GK-V 3216.

L ocus typicus: Bei der Talspere von Ooyubari, Yubari-Kohlenfeld, Hokkaido.

Stratigraphisches Verhalten: Diese Form wurde bisher nur im Feinsandstein der oberen Hakobuchi-Schichtengruppe (Maastricht) bei der Talspere von Ooyubari im Yubari-Kohlenfeld, Hokkaido gefunden. Diese Spezies wurde in der unteren Hakobuchi-Schichtengruppe (Campan) nicht gefunden.

Vergle i ch: Die quadrinus-Form ist der quadrilobus-Form (G. E. Rouse, 1957, S. 371, Taf. 2, Fig. 8-9) aus den oberkretazeischen Brazeau-Schichten von Alberta (Kanada) ähnlich. Die erstere ist auch der matsumotoi-Form (in dieser Arbeit, Taf. 41, Fig. 1-3) aus den oberkretazeischen Unter-HakobuchiSchichten von Hatsune-Sawa im Yubari-Kohlenfeld morphologisch ähnlich. Die quadrinus-Form kann nach der Skulptur der Exine und der Form des Vorsprungs von den zwei letzteren leicht unterschieden werden.

Die quadrinus-Form ist Aquilapollenites sp. bund c in dieser Arbeit (Taf. 15, Fig. 7a-c, 8a-c) ähnlich, aber die erstere kann nach Form des Vorsprungs und Skulptur de rExine von der Aquilapollenites sp. b-Form und der Aquilapollenites sp. c-Form unterschieden werden.

Aquilapollenites murus StANley (1961, S. 347, Taf. 6, Fig. 1-9) besitzt striate Exine. Diese Form hat E. A. STANLEY aus den maastrichtischen Hell 
Creek Schichten von Crow Butte, South Dakota, USA beschrieben. Sie ist kleiner als die quadrinus-Form. Ihre striate Skulptur ist etwas anderere Form als bei der quadrinus-Form.

Botanische Zugehörigkeit: fraglich.

\section{Aquilapollenites sp. a}

Taf. 37, Fig. 5a-b.

Isopolar. Körper $40 \mu$ groß. Figura wie Frucht der Wassernuß. Polachse ist eine dreizählige Symmetrieachse. Drei gespitzte, ohrenförmige Vorsprünge befinden sich je entsprechend in der Mitte der paarigen Halbcolpi, die nach den Pollen meridional verlaufen. Vorsprung etwas $10 \mu$ lang, dreieckig. Zwischen jedem Vorsprung verlaufen drei linienförmige Colpi äquatorial. Exine fein reticulat.

Die Form wurde nur im Kohlenflöz der unteren Hakobuchi-Schichtengruppe (Campan) von Hatsune-Sawa im Yubari-Kohlenfeld, Hokkaido gefunden.

Sie ist der triauritus- und borealis-Form morphologisch ähnlich, aber die erstere besitzt drei paarige Halbcolpi und drei äquatoriale Colpi. Die amerikanische Spezies mit den gleichen Eigentümlichkeiten, Aquilapollenites novacolpites Funkhouser (1961, S. 196, Taf. 2, Fig. 2a-c, 3) aus den Lance-Schichten (Maastricht) von Rawlins, Wyoming (USA), besitzt größere und mehr rundliche Vorsprünge als die vorliegende Form.

Botanische Zugehörigkeit: fraglich.

\section{Aquilapollenites sp. b}

Taf. 37, Fig. 7a-c.

Isopolar. Polachse vermutlich $\pm 50 \mu$ lang. Drei flügelförmige Vorsprünge ca. 15-16 $\mu$ lang, ca. $14 \mu$ breit, einer davon von etwas anderer, dreieckiger Form. Zwei subrechteckige Vorsprünge mit der rundlichen oder etwas truncaten Spitze besitzen je paarige, den Seiten fast parallel verlaufende lang-elliptische Halbcolpi, die ca. $9-10 \mu$ lang sind. Exine striat, wie Fingerabdrücke.

Diese Spezies fand sich nur im Kohlenflöz der unteren Hakobuchi-Schichtengruppe (Camapn) von Hatsune-Sawa im Yubari-Kohlenfeld, Hokkaido. Sie ist der quadrinus-Form und Aquilapollenites sp. c in dieser Arbeit ähnlich.

Botanische Zugehörigkeit: fraglich.

\section{Aquilapollenites sp. c \\ Taf. 37, Fig. 8a-c.}

Isopolar. Polachse vermutlich $\pm 46 \mu$ lang, dreizählige Symmetrieachse. Drei subrechteckige und flügelförmige Vorsprünge mit der etwas truncaten Spitze sind ca. $16.4 \mu$ lang und besitzen paarige den Seiten fast parallel verlaufende Halbcolpi. Exine striat, wie Fingerabdrücke.

Diese Form fand sich bisher nur im Kohlenflöz der unteren Hakobuchi- 
Schichtengruppe (Campan) von Hatsune-Sawa im Yubari-Kohlenfeld, Hokkaido. Sie ist Aquilapollenites sp. b morphologisch ähnlich, aber die erstere kann nach der Form eines Vorsprungs von der letzteren unterschieden werden.

Botanische Zugehörigkeit: fraglich.

\section{Aquilapollenites sp. d}

Taf. 38, Fig. 2a-b.

Isopolar. Polachse $52.5 \mu$ lang, dreizählige Symmetrieachse. Drei subrechteckige und flügelförmige Vorsprünge $19 \mu \mathrm{lang}, 17 \mu$ breit. Zwischengebiete der Pole und der Spitzen der äquatorialen Vorsprünge tief konkav; dort drei paarige Halbcolpi vorhanden. Sechs Halbcolpi linienförmig oder lang-elliptisch, etwa $11 \mu$ lang. Die drei Vorsprünge besitzen eine klein-papillate Skulptur (ewas $2 \mu$ lang). Ihre papillaten Warzen ordnen sich an den Spitzen der Vorsprünge und in der der Seitenlinie parallel verlaufenden Mittelzone an. Exine der Pol-Vorsprünge rugulat. Skulptur der sonstigen Exine fein punctat.

Diese Form wurde bisher nur im Kohlenflöz der unteren Hakobuchi-Schichtengruppe (Campan) von Hatsune-Sawa im Yubari-Kohlenfeld, Hokkaido gefunden. Sie ist den Arten Aquilapollenites matsumotoi TAKahashi (in dieser Arbeit, Taf. 38, Fig. 1a-b, 3a-b) und Aquilapollenites attenuatus FunkHouser (1961, S. 195-196, Taf. 2, Fig. 1a-c) aus den Lance-Schichten (Maastricht) von Rawlins, Wyoming (USA), sehr ähnlich. Die vorliegende Form kann nach der feinen Skulptur der Exine und dem Ornament des Pol-Vorsprungs von der matsumoto-Form und nach dem Ornament des Pol-Vorsprungs von der attenuatusForm untercshieden werden.

Botanische Zugehörigkeit: fraglich.

\section{? Aquilapollenites sp.}

Taf. 37, Fig. 6.

Es ist zweifelhaft, ob die vorliegende Form zu Aquilapollenites gehört oder nicht.

Ca. $22 \mu$ groß. Nur zwei kleine und rundliche Vorsprünge beobachtet. Exine schwach glatt.

Diese Form fand sich nur im Kohlenflöz der unteren Hakobuchi-Schichten von Hatsune-Sawa im Yubari-Kohlenfeld, Hokkaido.

Sie ist Aquilapollenites triauritus Takahashi (in dieser Arbeit, Taf. 37, Fig. 1a-c, 2a-c) morphologisch ähnlich, aber die erstere kann nach Größe und Skulptur von der letzteren leicht unterschieden werden.

Botanische Zugehörigkeit: fraglich.

Formgattung: Pentapollenites KRUTzscH

1957. Pentapollenites KrUTzSch, S. 520 . 


\section{Pentapollenites yezoensis n. sp. \\ Taf. 36, Fig. 28-31.}

D i a g n o s e : Ca. 22.3-25 $\mu$ groß. Isopolar. Polansicht dreieckig mit schwach konvexen Seiten. Beide Pole etwas vorgestülpt. Polachse kurz, dreizählige Symmetrieachse. Drei Germinalien an den Ecken nur klein, 2 bis $2.7 \mu$ tief. Atrium erkennbar. Linienförmiger Colpus bis fast $\mathrm{zu}$ den Polen reichend. Exine dünn, 0.5 bis $1 \mu$ dick, chagrenat.

H o l o ty p u s : Ca. $25 \mu$ groß; Taf. 36, Fig. 29 ; Präparat GK-V 3132.

L ocus typicus: Hatsune-Sawa, Ooyubari, Yubari-Kohlenfeld, Hokkaido.

Stratigraphisches Verhalten: Diese merkwürdige Form wurde nur im Kohlenflöz der unteren Hakobuchi-Schichtengruppe (Campan) von Hatsune-Sawa im Yubari-Kohlenfeld, Hokkaido gefunden. Sie tritt sehr selten auf.

Vergle i ch: Die vorliegende Form ist der Gattung Cupanieidites oberflächlich ähnlich, aber sie ist auch der mitteleuropäischen Form, Pentapollenites KRUTZSCH (1957, S. 520, Taf. 10, Fig. 1-4, 8, 9), morphologisch ähnlich. Die erstere weicht von der letzteren in einigen Merkmalen ab.

Botanische Zugehörigkeit: fraglich.

Formgattung: Pistillipollenites Rouse

1962. Pistillipollenites Rouse, S. 206.

\section{? Pistillipollenites sp.}

Taf. 41, Fig. 20a, b.

$21 \mu$ groß. Kontur rundlich. Figura kugelig. Breitenlängenindex ca. 0.87 . Colpus(?). Porus(?). Warzen (verrucat bis clavat) in lichter und unregelmäßiger Anordnung, $\pm 2 \mu$ hoch. Exine dünn.

Es ist zweifelhaft, ob die vorliegende Form zu Pistillipollenites gehört oder nicht; der Verfasser kann sie daher hier nur als ? Pistillipollenites sp. bezeichnen.

Sie wurde nur im Sandstein der oberen Hakobuchi-Schichtengruppe bei der Talspere von Ooyubari, Hokkaido gefunden.

Botanische Zugehörigkeit: Fraglich.

\section{B. Sporen und Pollen der Noborikawa-Schichten.}

Hier werden nur einige Formen, die in der Hakobuchi-Schichtengruppe nicht gefunden wurden, beschrieben.

\section{Sporites}

Triletes

Formgattung: Microreticulatisporites (KNOX) R. PotoniÉ \& KREMP

1950. Microreticulatisporites KNox, S. 320 .

1955. Microreticulatisporites (KNox) ex Potonít \& KREMP, S. 96. 


\title{
Microreticulatisporites sp. \\ Taf. 43, Fig. 1.
}

Trilete Spore. $33.4 \mu$ groß. Äquatorkontur dreieckig mit konvexen Seiten und abgerundeten Ecken. Y-Leisten schmal, den Äquator fast erreichend. Torus. Exine reticulat mit kleinen Lumina, deren Durchmesser $\pm 1 \mu$.

Die vorliegende Form wurde nur im Kohlenflöz der Noborikawa-Schichten bei der Talspere von Ooyubari im Yubari-Kohlenfeld, Hokkaido gefunden.

Botanische Zugehörigkeit: fraglich.

\section{Pollenites}

Aletes

Formgattung: Inaperturopollenites THomson \& PFLUG

1953. Inaperturopollenites THOMSON \& PFLUG, S. 64.

\author{
Inaperturopollenites shikokuensis TAKAHASHI \\ Taf. 43, Fig. 16.
}

1962. Inaperturopollenites shikokuensis, K. TAKAHASHI, S. 14, Taf. 3, Fig. 11-13.

Die vorliegende Figur ist $30 \mu$ groß. Form ursprünglich kugelig. Exine dünn, grob punctat. Skulptur stärker als bei der pseudodubius-Form. Exine einschichtig.

Sie fand sich nur im Kohlenflöz der Noborikawa-Schichten bei der Talspere von Ooyubari im Yubari-Kohlenfeld, Hokkaido.

Sie ist der pseudodubius-Form sehr ähnlich.

Botanische Zugehörigkeit: Salicaceae oder Triuridaceae kommen in Frage.

\section{Saccites}

Formgattung: Piceaepollenites R. PoToNIÉ

1958. Piceaepollenites R. Potoní, S. 64.

Piceaepollenites sp.

Taf. 43, Fig. 23a, b.

Ca. $100 \mu$ groß. Luftsäcke halbkugelig. Kamm nicht erkennbar. Stäbchen in den Luftsäcken chagrenat.

Die vorliegende Form ist nicht gut erhalten. Sie fand sich im Kohlenflöz der Noborikawa-Schichten bei der Talspere von Ooyubari im Yubari-Kohlenfeld, Hokkaido.

Botanische Zugehörigkeit: Picea. 


\section{Poroces}

Formgattung: Triporopollenites Thomson \& PfLug

1953. Triporopollenites Thomson \& PfLUG, S. 82.

\section{Triporopollenites shimensis TAKAHASHI}

Taf. 43, Fig. 9-11.

1961. Triporopollenites shimensis, K. TAKAHASHI, S. 301-302, Taf. 20, Fig. 5-14.

Ca. $33-40 \mu$ groß. Kontur abgerundet dreieckig. Keine Anulusbildung. Kein oder schwaches Labrum. Keine Tumeszenz. Eine Pore liegt meist subäquatorial. Kreisrunde oder schwach äquatorial gestreckte Pore. Exine dünn, chagrenat.

Die vorliegenden Figuren können von der Form des westjapanischen Alttertiärs und Miozäns nicht unterschieden werden.

Sie wurden im Kohlenflöz der Noborikawa-Schichten bei der Talspere von Ooyubari im Yubari-Kohlenfeld, Hokkaido gefunden.

Botanische Zugehörigkeit: Betulaceae.

\section{? Triporopollenites sp. \\ Taf. 43, Fig. 5.}

Ca. $24.5 \mu$ grof. Kontur dreieckig mit abgerundeten Ecken. Keine Anulusbildung und Tumeszenz. Kein Labrum. Pore klein. Exine dünn, chagrenat.

Die betreffende Form fand sich nur im Kohlenflöz der Noborikawa-Schichten bei der Talspere von Ooyubari im Yubari-Kohlenfeld, Hokkaido.

Botanische Zugehörigkeit: fraglich.

Formgattung: Betulaepollenites R. PoTONIÉ

1934. Betulaepollenites R. Potoní, S. 58.

1960. Betulaepollenites R. РотоNIE, S. 115.

\section{Betulaepollenites sp.}

Taf. 43, Fig. 4a, b.

$30 \mu$ groß. Kontur rundlich-dreieckig mit stark konvexen Seiten. Poren kreisrund, meridional nicht so tief. Anulus mit dem tropfenförmigen Querschnitt. Arci stark ausgeprägt; an der Polansicht verlaufen die Arci etwas bogenlinig von Exitus zu Exitus. Exine $1 \mu$ dick, chagrenat.

Die betreffende Form hat man nur im Kohlenflöz der Noborikawa-Schichten bei der Talspere von Ooyubari im Yubari-Kohlenfeld, Hokkaido gefunden.

Botanisch Zugehörigkeit: Betulaceae.

\section{Formgattung: Ulmipollenites WoLFF}

1934. Ulmipollenites WoLFF, S. 75. 


\section{Ulmipollenites undulosus WoLFF}

Taf. 43, Fig. 13.

1934. Ulmi-pollenites undulosus, WolfF, S. 75, Taf. 5, Fig. 25.

1953. Polyporopollenites undulosus, Thomson \& Pflug, S. 91, Taf. 10, Fig. 52-58.

1961. Polyporopollenites undulosus, K. TAKahashi, S. 308, Taf. 20, Fig. 15-18.

H. WOLFF (1934) hat Ulmi-pollenites undulosus WoLfF monotypisch beschrieben. Daher ist die Formgattung Ulmipollenites monenklatorisch gültig.

Die vorliegende Form kann von dem originalen Material von WoLfF und der Form aus dem Alttertiär und Miozän Westjapans nicht unterschieden werden.

Sie wurde im Kohlenflöz der Noborikawa-Schichten bei der Talspere von Ooyubari im Yubari-Kohlenfeld, Hokkaido gefunden.

Botanische Zugehörigkeit: Ulmus und Zelkova.

\section{Formgattung: Periporopollenites THomson \& PFLUG}

1937. Liquidambar-pollenites RAATZ, S. 17.

1953. Periporopollenites Thomson \& PFLug, S. 111.

1960. Liquidambarpollenites RAatz in R. Potonie, S. 134.

R. Potonié (1931) hat Pollenites stigmosus Potoniś nur mit einigen Bezeichnungen mitgeteilt. Er hat 1934 diese stigmosus-Form beschrieben. G. V. RAATZ (1937) hat keine neue Spezies unter der Gattung Liquidambarpollenites beschriebn. Die Gattung Periporopollenites wurde von P. W. THOMsoN und H. D. PFLUG nomenklatorisch gültig beschrieben.

\section{Periporopollenites asiaticus TAKAHASHI}

Taf. 44, Fig. 43-44.

1961. Periporopollenites asiaticus, K. TAKAHASHI, S. 332-333, Taf. 26, Fig. 34-36; Taf. 27, Fig. 1-9.

1962. Liquidambar asiatica, K. TAKAHASHI, S. 20, Taf. 5, Fig. 10-15.

Die Figuren sind sehr stark verfaltet. Figura ursprünglich kugelig. Kreisrunde oder elliptische foveenartige Exoporen sind in lichter Anordnung auf der Oberfläche verteilt. Exine sehr fein reticulat.

Die vorliegende Form ist mit der Form des westjapanischen Alttertiärs und Miozäns gleich. Sie fand sich im Kohlenflöz der Noborikawa-Schichten bei der Talspere von Ooyubari im Yubari-Kohlenfeld, Hokkaido.

Botanische Zugehörigkeit: Liquidambar.

\section{Plicates}

Triptyches

\section{Formgattung: Tricolpopollenites THOMsoN \& PFLUG}

1953. Tricolpopollenites Thomson \& Pflug, S. 95.

1960. Cornaceoipollenites R. РотоNIE, S. 93. 


\section{Tricolpopollenites vulgaris TAKAHASHI \\ Taf. 44, Fig. 22, 40.}

1957. Tricolpopollenites vulgaris, K. TAKahashI, S. 218, Taf. 38, Fig. 44-45; Taf. 39, Fig. 38.

1961. Tricolpopollenites vulgaris, K. Takahashi, S. 312, Taf. 22, Fig. 32-37.

Figura spindleförmig. Polkappenkontur zugespitzt. Drei Colpi verlaufen von Pol zu Pol. Schmale Cavernae konvergieren polwärts. Exine dünn, schwach chagrenat.

Diese Form wurde bisher im Alttertiär von Westjapan häufig gefunden. Sie fand sich im Kohlenflöz der Noborikawa-Schichten bei der Talspere von Ooyubari im Yubari-Kohlenfeld, Hokkaido.

Botanische Zugehörigkeit: Vielleicht Cupuliferen.

\section{Tricolpopollenites inamoenus TAKAHASHI \\ Taf. 44, Fig. 23.}

1961. Tricolpopollenites inamoenus, K. TAKaHASHI, S. 313, Taf. 22, Fig. 42-49.

$26.6 \mu$ groß. Kontur elliptisch. Figur ellipsoidisch. Breitenlängenindex ca. 0.7. Exine schwach verrucat, $\pm 0.5 \mu$ hoch, in verhältnismäßig dichter Anordnung. Polkappenkontur etwas zugespitzt.

Diese Spezies konnte man im Kohlenflöz der Noborikawa-Schichten bei der Talspere von Ooyubari im Yubari-Kohlenfeld, Hokkaido finden. Sie ist eine sehr stratigraphisch wichtige Art, die im westjapanischen Alttertiär, besonders im Obereozän (Noogata-Pollenbild) häufig aufgetreten ist.

Botanische Zugehörigkeit: Cupuliferen (?).

\section{Tricolpopollenites reticulatus TAKAHASHI}

Taf. 44, Fig. 34-36.

1961. Tricolpopollenites reticulatus, K. TAKaHASHI, S. 318-319, Taf. 23, Fig. 44-49.

Figura kugelig. Breitenlängenindex ca. 1. Polhemisphäre halbkugelig. Drei Colpi konvergieren polwärts. Fein baculate Skulptur. Die Stäbchen stehen locker innerhalb reticulater Zwischenräume geordnet. Vieleckiges Netz über $1 \mu$ breit.

Diese Art wurde im Kohlenflöz der Noborikawa-Schichten bei der Talspere von Ooyubari im Yubari-Kohlenfeld, Hokkaido gefunden. Man konnte bisher sie im Alttertiär von Westjapan häufig finden.

Botanische Zugehörigkeit: Salix, Platanus u. a.

\section{Tricolpopollenites rudis TAKAHASHI}

Taf. 44, Fig. 1-4.

1961. Tricolpopollenites rudis, K. TAKAHASHI, S. 319-320, Taf. 24, Fig. 1-13. 
33-42 $\mu$ groß. Figura kugelig. Breitenlängenindex \pm 1 . Polhemisphäre halbkugelig bis unterhalbkugelig. Sehr schmale, nahezu parallel verlaufende Cavernae. Exine verhältnismäßig fein reticulat.

Diese rudis-Form ist eine paläogene Spezies in Westjapan.

Sie wurde in der Sasebo-Schichtengruppe von Nordkyushu gar nicht gefunden.

Hier in Hokkaido wurde sie im Kohlenflöz der Noborikawa-Schichten bei der Talspere von Ooyubari im Yubari-Kohlenfeld verhältnismäßig häufig gefunden.

Botanische Zugehörigkeit: fraglich.

Tricolpopollenites minutissmus n. sp.

Taf. 44, Fig. 5-10.

D i a $\mathrm{g}$ n o s e : Sehr kleiner tricolpater Pollen. Ca. 10-11 $\mu$ groß. Figura \pm kugelig. Breitenlängenindex 0.8 bis 0.9 . Polhemisphäre halbkugelig bis unterhalbkugelig. Drei Colpi verlaufen \pm parallel und konvergieren sehr schwach polwärts. Drei Cavernae sehr schmal. Exine sehr dünn, unter $0.5 \mu$ dick, chagrenat.

H o l ot y p u s : $10.5 \mu$ groß; Taf. 44, Fig. 7; Präparat GK-V 3203.

Locus typicus: Kohlenflöz (J) der Noborikawa-Schichten, bei der Talspere von Ooyubari im Yubari-Kohlenfeld, Hokkaido.

Stratigraphisches Verhalten: Diese Form wurde nur im Kohlenflöz der Noborikawa-Schichten bei der Talspere von Ooyubari im YubariKohlenfeld gefunden.

Vergle i ch: Die minutissmus-Form ist kleiner und mehr kreisrundlich als die liblarensis fallax-Form (R. PotonıÉ, 1934, S. 70, Taf. 3, Fig. 10; P. W. Thomson \& H. D. Pflug, 1953, S. 97, Taf. 11, Fig. 133-151; K. Takahashi, 1961, S. 314, Taf. 23, Fig. 5-11) . Die erstere ist auch der Spezies, Tricolpopollenites subasper Takahashi (1957, S. 217-218, Taf. 38, Fig. 42-43; 1961, S. 314, Taf. 23, Fig. 12-14), ähnlich, aber die letztere ist größer.

Botanische Zugehörigkeit: fraglich.

Tricolpopollenites sp.

Taf. 44, Fig. 33.

Tricolpater Pollen. $21.5 \mu$ groß. Figura spindelförmig. Breitenlängenindex ca. 0.7. Drei Colpi konvergieren polwärts. Caverna schmal. Exine sehr dünn, fein punctat.

Diese Spezies wurde nur im Kohlenflöz der Noborikawa-Schichten bei der Talspere von Ooyubari im Yubari-Kohlenfeld gefunden.

Botanische Zugehörigkeit: fraglich. 


\title{
Formgattung: Rhoipites WoDEHOUSE
}

1933. Rhoipites WodEHOUSE, S. 513.

1960. Rhoipites WodeHouse in R. Potonie, S. 100-101.

\author{
Rhoipites ? hoshuyamaensis (TAKAHASHI) n. comb. \\ subsp. hoshuyamaensis n. emend. \\ Taf. 44, Fig. 41.
}

Synonym: Tricolporopollenites hoshuyamaensis TAKAHASHI subsp. fossulatus TAKAHASHI, 1961, S. 325, Taf. 25, Fig. 5-9.

Der Verfasser (1961) hat Tricolporopollenites hoshuyamaensis beschrieben. Dabei hat er zwei Subspezies, fossulatus und foveolatus, vorgeschlagen. Nach dem Internationalen Code (Artikel 24 und 26) muß der Name Rhoipites ? hoshuyamaensis (TAKAHASHI) subsp. hoshuyamaensis statt des erstmaligen Namens Tricolporopollenites hoshuyamaensis TAKAHASHI subsp. fossulatus TAKAHASH als gültig gegeben werden.

Die Figur 41 ist nicht gut erhalten. Sie kann mit der hoshuyamaensis-Form, die im eozänen Schichten von Westjapan gefunden wurde, verglichen werden.

Tricolporater Pollen. Figur ellipsoidisch (?). Polkappenkontur zugespitzt. Drei Colpi verlaufen von Pol zu Pol. und konvergieren polwärts. Cavernae schmal. Undeutlicher Porus, wenig über die Caverna hinausgreifend (?). Exine fossulat.

Die hoshuyamaensis-Form ist in den eozänen Schichten Westjapans beschränkt aufgetreten. Sie ist eine stratigraphisch wichtige Art.

Nur ein Pollenkorn dieser Form wurde im Kohlenflöz der NoborikawaSchichten bei der Talspere von Ooyubari im Yubari-Kohlenfeld, Hokkaido gefunden.

Botanische Zugehörmigkeit: fraglich.

Formgattung: Tricolporopollenites Thomson \& PfLUG

1953. Tricolporopollenites Thomson \& PfLug, S. 98.

Tricolporopollenites incertus TAKAHASHI

Taf. 44, Fig. 29.

1961. Tricolporopollenites incertus, K. TAKahashi, S. 322-323, Taf. 24, Fig. 50-52.

Tricolporater Pollen. Figur ellipsoidisch. Polhemisphäre etwa halbkugelig. Drei Colpi erreichen fast die Pole. Schmale Cavernae verlaufen polwärts. Der Hauptporus ist meridional gestreckt. Der Porus wird von den Caverna umschrieben. Exine sehr dünn, chagrenat.

Diese Form wurde im Kohlenflöz der Noborikawa-Schichten bei der Talspere von Ooyubari im Yubari-Kohlenfeld, Hokkaido gefunden.

Botanische Zugehörmigkeit: fraglich. 
Tricolporopollenites cf. cingulum (R. Pот.) THOMson \& PFLUG subsp. fusus (R. Pот.) ThOMson \& PfLUg

Taf. 44, Fig. 24-25.

1931. Pollenites fusus, R. Potonie, S. 332, Tab. 1, Fig. 13.

1953. Tricolporopollenites cingulum fusus, P. W. Thomson \& H. D. Pflug, S. 100, Taf. 12, Fig. 15-27.

1961. Tricolporopollenites cingulum fusus, K. TAKAHASHI, S. 321-322, Taf. 24, Fig. $42-43$.

Figur ellipsoidisch. Polhemisphäre zugespitzt. Cavernae \pm parallel, bis zu den Polen verlaufend. Exine dünn, fein intrarugulat (?). Hauptporus ist eine kleine Ruge.

Die fusus-Form ist eine europäische tertiäre Form, aber sie tritt den paläogen und miozänen Schichten von Westjapan auf. Der Verfasser hat im Kohlenflöz der Noborikawa-Schichten bei der Talspere von Ooyubari im Yubari-Kohlenfeld gefunden.

Botanische Zugehörigkeit: Castanea?

\section{Tricolporopollenites castaneoides TAKAHASHI}

Taf. 44, Fig. 26-28.

1961. Tricolporopollenites castaneoides, K. TAKAHASHI, S. 321, Taf. 24, Fig. 34-40.

Tricolporater Pollen. Figura ellipsoiidsch oder spindelförmig. Polhemisphäre zugespitzt. Colpen erreichen fast die Pole. Cavernae \pm parallel. Hauptporus ist eine kleine Ruge. Exine dünn, unter $1 \mu$ dick, schwach glatt bis chagrenat.

Die castaneoides-Form fand sich bisher in den westjapanischen paläogenen und miozänen Schichten selten. Man kann sie im Kohlenflöz der NoborikawaSchichten bei der Talspere von Ooyubari im Yubari-Kohlenfeld, Hokkaido wenig finden.

Botanische Zugehörigkeit: Castanea.

Formgattung: Ilexpollenites (ThIERGART) R. POTONIÉ

1937. Ilexpollenites THIERGART, S. 321 .

1960. Ilexpollenites (ThieRgart) ex R. Potonie, S. 99-100.

Ilexpollenites excellens (TAKAHASHI) n. comb.

Taf. 43, Fig. 14.

1961. Tricolporopollenites excellens, K. TAKAhaShI, S. 331-332, Taf. 26, Fig. 24, 26, 28.

Ca. $29 \mu$ groß. Figur kugelig. Die Höhe der Keulchen zwischen 1/12 und $1 / 10$ des maximalen Korndurchmessers. Clavae seitlich verdickt. Der Durchmesser der Clavae bis $2 \mu$. Keulchen $2.8 \mu$ hoch. Exine gleichmäßig mit Keulchen besetzt. Endoporus undeutlich. 
Die vorliegende Figur wurde im Kohlenflöz der Noborikawa-Schichten bei der Talspere von Ooyubari im Yubari-Kohlenfeld, Hokkaido gefunden. Die excellens-Form hat man bisher in den alttertiären und miozänen Schichten von Westjapan wenig bemerkt.

Botanische Zugehörigkeit: Ilex.

Ilexpollenites clavatus (TAKAHASHI) n. comb.

Taf. 43, Fig. 15.

1961. Tricolporopollenites clavatus, K. TAKahashi, S. 331, Taf. 26, Fig. 12-15, 25, 27.

Tircolporater Pollen. $29 \mu$ groß. Figur breit-ellipsoidisch. Die Höhe der Keulchen mehr als $1 / 10$ des maximalen Korndurchmessers. Clavae $\pm 3 \mu$. Keulenhöhe $4.5 \mu$. Exine gleichmäßig mit Keulchen besetzt. Endoporus undeutlich.

Die clavatus-Form wurde bisher in den westjapanischen alttertiären und miozänen Schichten spärlich gefunden. Hier in Hokkaido ist sie aud dem Kohlenflöz der Noborikawa-Schichten bei der Talspere von Ooyubari im YubariKohlenfeld aufgetreten.

Botanische Zugehörigkeit: Ilex.

Jugates

Formgattung: Ericipites WoDEHouse

1933. Ericipites WodeHouse, S. 516.

1953. Tetradopollenites Thomson \& PfLug, S. 112.

1960. Ericipites Wodehouse in R. PotonIE, S. 137-138.

\section{Ericipites sp.}

Taf. 44, Fig. 42.

Tetrade. Ca. $31.3 \mu$ groß. Exine dünn. Ekt- und Endexine gleichstark. Jedes Korn mit kurzem Colpus.

Die Pollenform, die zu den Ericaceae gehört, wurde bisher in den alttertiären und miozänen Schichten von Westjapan spärlich bemerkt.

Dit vorliegende Form fand sich im Kohlenflöz der Noborikawa-Schichten bei der Talspere von Ooyubari im Yubari-Kohlenfeld, Hokkaido.

Botanisch Zugehörigkeit: Ericaceae.

\section{Literaturverzeichnis}

Balme, B. E. (1957): Spores and pollen grains from the Mesozoic of western Australia (Catalog of fossil spores and pollen, vol. 16). Commonwealth Sci. Ind. Res. Organization, coal Res. Sect., T. C. 25, (48), pls. 11, figs. 128, tabs. 4.

Balme, B. E. and Hemelly, J. P. F. (1955): Bisaccate sporomorphs from Australian Permian coals. (Catalog of fossil spores and pollen, vol. 13). Aust. Jour. Bot., 3, (1), 89-98, pls. 1-6.

BERrY, W. (1937): Spores from the Penuington coal, Rhea county, Tennessee. (Catalog of 
fossil spores and pollen, vol. 7). Am. Midland Naturalist, 18, 155-160, figs. 14.

BhARDwAJ, D. C. (1957): The palynological investigations of the Saar coals (Part I-Morphography of Sporae dispesae). Palaeontographica, B, 101, 73-125, pls. 22-31, text-fig. 7 , tab. 1 .

-, (1957): The spore flora of Velener Schichten (Lower Westphalian D) in the Ruhr coal measures. Palaeontographica, B, 102, 110-138, pls. 23-26, text-figs. 9, tab. 3.

Bolkhovitina, N. A. (1953): Spores and pollen characteristic of Cretaceous deposits of central regions of the U. S. S. R. (Catalog of fossil spores and pollen, vol. 8). Trans. Inst. Geol. Acad. Sci. USSR, 145, Geol. Ser. (61), 1-184, pls. 16, figs. 10, tabs. 10.

- (1956): Atlas of spores and pollen from Jurassic and lower Cretaceous deposits of the Vilyui depression. (Catalog of fossil spores and pollen, vol. 10). Trans. Geol. Inst. Acad. Sci. USSR, (2), 1-188, pls. 25, text-figs. 9, tabs. 4.

-, (1961): Fossil and recent spores of the family Schizaeaceae. Trans. Geol. Inst. Acad. Sci. USSR, (40), 1-176, pls. 41, text-figs. 10.

BRADley, W. H. (1931): Origin and microfossils of the oil shale of the Green River formation of Colorado and Utah. U. S. Geol. Surv. Prof. Paper, 168, 1-58.

Cookson, I. C. (1947): Plant microfossils from the lignites of Kerguelen Archipelago. (Catalog of fossil spores and pollen, vol. 15), B.A. N.Z. Antarctic Research Expedition 19291931, Rpts.-Series $A$, 2, (8), 127-142, pls. 13-17.

- , (1947): On fossil leaves (Oleaceae) and a new type of fossil pollen grain from Australian brown coal deposits. (Catalog of fossil spores and pollen, vol. 15). Proc. Limn. Soc. New South Wales, 72, (3-4), 183-197, pls. 8-10, text-figs. 4.

(Catalog of fossil spores and pollen, vol. 15). Australian Jour. Sci., Research Ser. B-Biol. Sci., 3, (2), 166-177, pls. 1-3, text-figs. 2 .

- (1953): Difference in microspore composition of some samples from a bore at Comaum, south Australia. (Catatog of fossil spores and pollen, vol. 15). Austr. Jour. Bot., 1, (3), 462-473, pls. 1-2, tab. 1 .

, (1954): The Cainozoic occurrence of Acacia in Australia. (Catalog of fossil spores and pollen, vol. 15). Austr. Jour. Bot., 2, (1), 52-59, pl. 1, tab. 1.

-, (1957): On some Australian Tertiary spores and pollen grains that extend the geological and geographical distribution of living genera. (Catalog of fossil spores and pollen, vol. 15). Proc. Roy. Soc. Victoria, 69, 41-53, pls. 8-10.

Cookson, I. C. and PIKE, K. M. (1953): A contribution to the Tertiary occurrence of the genus Dacrydium in the Australian region. (Catalog of fossil spores and pollen, vol. 15). Austr. Jour. Bot., 1, (3), 474-484, pls. 1-3, Tab. 1.

and - (1954): Some dicotyledonous pollen from Cainozoic deposits in the Australian region. (Catalog of fossil spores and pollen, vol. 15). Australian Jous. Bot., 2, (2), 197-219, pls. 1-2, text-fig. 1, tabs. 2 .

$\longrightarrow$ and $-(1954)$ : The fossil occurrence of Phyllocladus and two other Podocarpaceous types in Australia. (Catalog of fossil spores and pollen, vol. 15). Australian Jour. Bot., 2, (1), 60-68, pls. 2.

Cookson, I. C. and Dettmann, M. E. (1958): Some trilete spores from upper Mesozoic deposits in the eastern Australian region. (Catalog of fossil spores and pollen, vol. 15). Proc. Roy. Soc. Victoria, New Ser., 70, (2), 95-128, pls. 14-19, fig. 1, tab. 1.

- and,$-(1958)$ : Cretaceous "Megaspores" and a closely associated microspore from the Australian region. Micropaleontology, 4, (1), 39-49, pls. 1-2, text-figs. 3, tab. 1.

$\longrightarrow$ and - (1959): On Schizosporis a new form genus from Australian Cretaceous deposists. Micropaleontology, 5, (2), 213-216, pl. 1.

Couper, R. A. (1953): Upper Mesozoic and Cainozoic spores and pollen grains from New Zealand. (Catalog of fossil spores and pollen, vol. 2). New Zealand Geol. Surv., Paleont, Bull., 22, 1-77, pls. 9, text-figs, 3, tabs. 3.

- (1954): Plant microfossils from New Zealand No. I. (Catalog of fossil spores and pollen, vol. 4). Trans. Roy. Soc. New Zealand, 81, (4), 479-483, figs. 9, text-fig. 1. 
Couper, R. A. (1958): British Mesozoic microspores and pollen grains. A systematic and stratigraphic study. Palaeontographica, B, 103, 75-179, pls. 15-31, figs. 11, tabs. 12 .

Daugherty, L. H. (1941): The upper Triassic flora of Arizona. (Catalog of fossil spores and pollen, vol. 8). Carnegie Inst. of Washington, Contr. Paleont. Pub., 526, 1-108, pls. 34.

Delcourt, A. et Sprumont, G. (1955): Les spores et grains de pollen Wealdien du Hainaut. (Catalog of fossil spores and pollen, vol. 4). Soc. Belge de Géol. de Paléont. Hydrolog., Mém. Nouv., Sér. in 4, (5), pl. 4, text-figs. 15, tabs. 3.

Endo, Seido (195): Nilssonia-bed of Hokkaido and its flora. Sci. Rep. Tohoku Imp. Univ., 2nd. ser. (Geol.), 7, (3), 57-72, pls. 11-17.

Erdtman, G. (1952): Pollen morphology and plant iaxonomy, Angiosperms. Chronica Botanica Co., Waltham, Mass.

- (1954): An introduction to pollen analysis. Chronica Botanica Co., Waltham, Mass. , (1948): Did dicotyledonous plants exist in early Jurassic times? (Catolog of fossil spores and pollen, vol. 9). Geol. Fören. I Stockholm Förh., 70, (2), 265-271, figs. 15.

, (1958): Palynology-development and growth. 4th sir Albert Charles Seward memorial lecture, 14 Nov. 1956. Birbal Sahni Inst. Palaeobotany.

- , (1960): Pollen walls and angiosperm phylogeny. Botaniska Notiser, 113, (1), 41-45.

- $(1960)$ : On three new genera from the lower Headon beds, Berkshire. Botaniska Notiser, 113, (1), 46-48, pls. 1-2.

FAegri, K. and Iversen, J. (1950): Text-book of modern pollen analysis. Copenhagen.

Funkhouser, J. W. (1961): Pollen of the genus Aquilapollenites. Micropaleontology, 7, (2), 193-198, pls. 1-2, text-fig. 1.

Groot, J. J. and PenNy, J. S. (1960): Plant microfossils and age of non marine Cretaceous sediments of Maryland Delaware. Micropaleontology, 6, (2), 225-236.

Groot, J. J., PEnNy, J. S. and GRoot, C. R. (1961): Plant microfossils and age of the Raritan, Tuscaloosa and Magothy formations of the eastern United States. Palaeontographica, B, 108, 121-140, pls. 24-26, fig. 1, tabs. 2.

Groot, J. J. and GROOT, C. R. (1962): Some plant microfossils from the Brightseat formation (Paleocene) of Maryland. Palaeontographica, B, 111, 161-171. pls. 29-31.

HiRMER, Max (1927): Handbuch der Paläobotanik. 622.

HoffMeister, W. S. (1959): Lower Silurian pland spores from Libya. Micropaleontology, 5, (3), 331-334, pl. 1, text-fig. 1.

IBRAHIM, A. C. (1933): Sporenformen des Aegirhorizontes des Ruhrreviers. (Catalog of fossil spores and pollen, vol. 6). Triltsch, Würzburg, 46 S. Taf. 8, Tab. 1.

IKUSE, Masa (1956): Pollen grains of Japan. (in Japanese), 303, pls. 1-76. Hirokawa Publishing Co., Tokyo.

Ishchenko, A. M. (1956): Spores and pollen of the lower Carboniferous deposits of the western extension of the Donets basin and their stratigraphic importance. (Catalog of fossil spores and pollen, vol. 7). Izd. A. N. Ukrainian SSR, stratigraphic and plaeontologic ser., Contr. 11, 1-185, pls. 20.

JoNAs, Fr. (1952): Atlas zur Bestimmung rezenter und fossiler Pollen und Sparen. Feddes Repert, Beiheft 133 .

Just, Theodor (1951): Mesozoic plant microfossils and their geological significance. Jour. Paleont., 25, (6), 729-735.

Kirchheimer, F. (1950): Mikrofossilien aus Salzablagerungen des Tertiärs. Palaeontographica, B, 90, 127-160.

KLAUS, W. (1955): Über die Sporendiagnose des deutschen Zechsteinsalzes und des alpinen Salzgebirges. Deutsch. Geol. Gesell. Zeitschrift, 105, 776-788, Taf. 33-34, Abb. 3.

KosANKe, R. (1950): Pennsylvanian spores of Illinois and their use in correlation. (Catalog of fossil spores and pollen, vol. 5). Illinois State Geol. Surv. Bull., 74, 128, pls. 18, text-figs. 7.

Kräusel, R. und Leschik, G. (1956): Die Keuperflora von Neuewelt bei Basel. II. Die Isound Mikrosporen. Schweizerische Paläont. Abh., 72, 1-70, Taf. 10, Abb. 1. 
KREMP, G. (1952): Sporen-Vergesellschaftungen und Mikrofaunen-Horizonte im Ruhrkarbon, III. (Catalog of fossil spores and pollen, vol. 6). Congrès Pour L'Avancement des Etudes de Stratigraphie et de Géologie du Carbonifére, Heerlen, 25-30 juin 1951, compte Rendue, Tome 1, 347-357, text-fig. 1-8, Taf. 15b, fig. 1-20.

KRUTzScH, Wilfried (1956): Zur Kenntnis und Bedeutung abnorm entwickelter fossiler Pollen und Sporen. Geologie, 5, (1), 30-41.

, (1956): Normapolles im sächsischen Turon. Jb. staatl. Mus. Min. Geol., 2, 125-131.

, (1957): Sporen- und Pollengruppen aus der Oberkreide und dem Tertiär Mitteleuropas und ihre stratigraphische Verteilung. Z. angewandte Geologie, Heft 11/12, 509-548, Taf. 1-16, Tab. 1-2.

, (1957): Sporenpaläontologische Untersuchungen in der sächsisch-böhmischen Kreide und die Gliederung der Oberkreide auf mikrobotanischer Grundlage. (2. Mitt.). Ber. Geol. Gas. DDR, 2, (2), 123-129.

- (1959): Einige neue Formgattungen und -arten von Sporen und Pollen aus der mitteleuropäischen Oberkreide und dem Tertiär. Palaeontographica, 105, 125-157, Taf. 31-35, Abb. 18.

Bemerkungen zur regionalen Verbreitung einiger Pollengruppen im älteren Paläogen. Freiberger Forschungshefte, Heft C 86, 54-65.

Kuyl, O. S., Muller, J. and Waterbolk, H. T. (1955): The application of palynology to oil geology, with special reference to western Venezuela. Geol. en Mijnbouw, NW. S., $17 \mathrm{e}$ Jg., 3, 49-75.

LANTz, J. (1958): Étude des Spores et pollens dún Échantillon Purbeckien de L'clle d'Oléron. Revue de Micropaléontologie, 1, (1), 33-37, pl. 1, figs. 21.

LeschiK, G. (1956): Sporen aus dem Salzton des Zechsteins von Neuhof (bei Fulda). Palaeontographica, B, 100, 122-142, Taf. 3.

Loose, F. (1934): Sporenformen aus dem Flöz Bismarck des Ruhrbegietes, (Catalog of fossil spores and pollen, vol. 6). Petrogr. Brennst., 4, 128-164, Taf. 7, text-figs. 2.

MANum, S. (1954): Pollen og sporer i tertiaere kull fra Vestspitsbergen. (Pollen and spores in Tertiary coal from West Spitsbergen). Meddelelser, (79), 1-9.

- (1960): On the genus Pityosporites Seward 1914 with a new description of Pityosporites antarcticus Seward. Nytt Magasin for Botanikk, 8, 11-15, pl. 1.

- (1962): Studies in the Tertiary flora of Spitsbergen, with notes on Tertiary floras of Ellesmere Island, Greenland and Iceland. Norsk Polarinstitutt, Skrifter Nr. 125, 1127, pls. $1-20$.

Matsumoto, Tatsuro (Editor) (1953): The Cretaceous system in the Japanese islands. 324, pls. 1-20, The Japan Society for the Promotion of Science, Tokyo.

- (1959): Zonation of the upper Cretaceous in Japan. Mem. Fac. Sci., Kyushu Univ., Ser. D, Geol, 9, (2), 55-93, pls. 6-11.

MEYer, B. L. (1956): Mikrofloristische Untersuchungen an jungtertiären Braunkohlen im östlichen Bayern. Geologica Bavarica, (25), 100-128, Taf. 5, Diagr. 2, Beilag 2.

Miner, E. L. (1935): Paleobotanical examination of Cretaceous and Tertiary coals. (Catalog of fossil spores and pollen, vol. 8). Amm. Midland Naturalist., 16, (4), 616-625, pls. 23-24, figs. 1-20.

MrtTre, Vishnu (1955): Sporojuglandoidites jurassicus gen. et sp. nov. A sporomorph from the Jurassic of the Rajmahal Hills, Bihar. The Palaeobotanist, 4, 151-152, figs. 3.

Mürriger, F. und Pflanzl, G. (1955): Pollenanalytische Datierung einiger hessischer Braunkohlen. Notizbl. hess. L-Amt Bodenforschung, 83, 71-89.

NAGAO, S., OSANAI, H. and SAKo, S. (1954): Explanatory text of the geological map of Japan. Scale 1:50.000 Oyubari. (in Japanese with English resumé). Hokkaido-Kaihatsu-Cho.

OKAzAKI, Y. (1957): Palynological and stratigraphical studies on the Paleogene coals in the Kushiro coal field, eastern Hokkaido. Geology of the Beppo formation and its pollenanalysis (pt. 1). Jour. Hokkaido Gakugei Univ., 8, (1), 91-97.

OLDFIELD, Frank (1959): The pollen morphology of some of the west european Ericales. 
Preliminary descriptions and a tentative key to their identification. Pollen et spores, 1, (1), 19-48, pls. 1-2, fig. 1, tab. 1.

Pflanzl, G. (1956): Das Alter der Braunkohlen des Meißners, der Flöze 2 und 3 des Hirschbergen und eines benachbarten Kohlenlagers bei Landenbach. Notizbl. hess. L-Amt Bodenforsch., 84, 232-244.

Pflug, H. D. (1952): Palynologie und Stratigraphie der eozänen Braunkohlen von Helmstedt. Paläont. Z., 26, 112-137, Taf. 6-8, Abb. 1-8.

-, (1953): Zur Entstehung und Entwicklung des angiospermiden Pollens in der Erdgeschichte. Palaeontographica, B, 95, 60-171.

, (1956): Beiträge zur Klimageschichte Islands II. Sporen und Pollen von Tröllatunga (Island) und ihre Stellung zu den pollenstratigraphischen Bildern Mitteleuropas. N. Jb. Geol. Paläont., Abh. 102, (3), 409-430, Taf. 17-19.

- (1959): Beiträge zur Klimageschichte Islands VIII. Sporenbilder aus Island und ihre stratigraphische Deutung. N. Jb. Geol. Paläont., Abh. 107, (2), 141-172, Taf. 12-16. Tab. 1-2.

Pocock, S. A. J. (1962): Microfloral analysis and age determination of strata at the JurassicCretaceous boundary in the western Canada plains. Pataeontographica, B, 111, 1-95, pls. 1-15, figs. 21 , tabs. 3 .

Pokrovskaya, I. M. ane Stel'MAK, N. K. (1960): Atlas of upper Cretaceous, Paleocene and Eocene spore-pollen complexes of several regions of the USSR. (Catalog of fossil spores and pollen, vol. 16). Trans. All-Soviet Scientific Research Geol. Inst. Ministry Geol. and Petroleum Conservation USSR, new ser., 30, 1-575, 87 pls.

Potoní́, R. (1931): Zur Mikroskopie der Braunkohlen. Tertiäre Blütenstaubformen. (1. Mitt.). (Catalog of fossil spores and pollen, vol. 2). Z. Braunkohle, 16, 30, 325-333, Taf. 2 .

—, (1931): Pollenformen der miozänen Braunkohle. (2. Mitt.). (Catalog of fossil spores and pollen, vol. 4). Sitz. Ber. Ges. Naturf. Fr., (1-3), 24-28.

- (1931): Pollenformen aus tertiären Braunkohlen. (3. Mitt.). (Catalog of fossil spores and pollen, vol. 1). Jb. Preuss. Geol. L-Amt Bodenf., 52, 1-7, fig. 34.

- (1931): Zur Mikroskopie der Braunkohlen Tertiäre Sporen- und Blütenstaubformen. (4. Mitt.). (Catalog of fossil spores and pollen, vol. 4). Z. Braunkohle, 27, 30, 554556, Abb. 16.

- - (1934): Zur Mikrobotanik des eozänen Humodils des Geiseltals. (Catalog of fossil spores and pollen, vol. 1). Arb. Inst. Palaeobot. u. Petrogr. Brennst. Preuss. Geol. $L-A m t, 4,25-125$, Taf. 1-6.

- (1947): Zum Stand der mikropaläobotanischen Tertiärstratigraphie. Geol. Rundschau, 37, 112-113.

- , (1950): Stand der mikropaläobotanischen Tertiärstratigraphie. Z. deutsch. Geol. Ges., 100, 366-378.

- (1951): Die Mikrobotanik der Kohle auf dem internationalen Botanikerkongress zu Stockholm im Juli, 1950. Geol. Rundschau, 39, (1), 247-249.

- (1951): Revision stratigraphisch wichtiger Sporomorphen des mitteleuropäischen Tertiärs. Palaeontographica, B, 91, 131-151.

- (1956): Synopsis der Gattungen der Sporae dispersae. I. Teil. Beih. Geol. Jb., 23, 1103, Taf. 1-11.

- (1958): Synopsis der Gattungen der Sporae dispersae. II. Teil. Beih. Geol. Jb., 31, 1-114, Taf. 11.

- , (1960): Synopsis der Gattungen der Sporae dispersae. III. Teil. Beih. Geol. Jb., 39, 1-189, Taf. 9 .

- (1960): Sporologie der eozänen Kohle von Kalewa in Burma, Senckenbergiana Lethaea, 41, (1/6), 451-481, Taf. 2, Abb. 12.

Potonié, R., Ibrahim, A., und Loose, F. (1932): Sporenformen aus den Flözen Ägir und Bismarck des Ruhrgebietes. (Catalog fo fossil spores and pollen, vol. 5). N. Jb. Minerl. Beil.-Bd. 67, B, 438-454, Taf. 14-20, text-fig. 1. 
Potonié, R. und Venitz, H. (1934): Zur Mikrobotanik des miozänen Humodils der niederrheinischen Bucht. (Catalog fo fossil spores and pollen, vol. 4). Arb. Inst. Paleobot. u. Petrogr. Bennst., 5, 5-54, Taf. 1-4, Abb. 2.

Potoní́, R. und Klaus, W. (1954): Einige Sporengattungen des alpinen Salzgebirges. Geol. $J b ., 68,517-546$, Taf. 10, Abb. 11.

Potoní, R. und Kremp, G. (1954): Die Gattungen der paläozoischen Sporae dispersae und ihre Stratigraphie. Geol. Jb., 69, 111-194, Taf. 17, Abb. 5.

- und $\longrightarrow,(1955):$ Die Sporae dispersae des Ruhrkarbons. Teil I. Palaeontographica, B, 98, 1-136, Taf. 1-16, Abb. 37 .

- und - (1956): Die Sporae dispersae des Ruhrkarbons. Teil II. Palaeontographica, B, 99, 85-191, Taf. 17-22, Abb. 38-88.

- und $-(1956):$ Die Sporae dispersae des Ruhrkarbons. Teil III. Paraeontographica, B, 100, 65-121, Tab. 3.

RAATZ, G. V. (1937): Mikrobotanisch-stratigraphische Untersuchung der Braunkohle des Muskauer Bogens. Abh. preuss. Geol. L-A. N. F., 183, 1-48, Taf. 1, Abb. 5.

RAUKopF, K. (1959): Pollenanalytische Untersuchungen zur Feinstratigraphie der Tertiärkohlen von Mecklenburg, Berlin und Lausitz. Abh. deutsch. Akademie Wissenschaften Berlin, (8), 3-24.

Reissinger, A. (1939): Die „, Pollenanalyse “ ausgedehnt auf alle Sedimentgesteine der geologischen Vergangenheit. Palaeontographica, B, 84, 1-20.

- , (1950): Die , Pollenanalyse" ausgedehnt auf alle Sedimentgesteine der geologischen Vergangenheit. Palaeontographica, B, 90, 99-126.

Ross, N. E. (1949): Investigations of the Senonian of the Kristianstad district, S. Sweden. I. On a Cretaceous pollen and spore bearing clay deposit of Scania. A preliminary report. Bull. Geol. Inst. Univ. Upsala, 34, 25-43, pls. 3, text-figs. 3.

Rouse, G. (1957): The application of a new nomenclatural approach to upper Cretaceous plant microfossils from western Canada. Can. Jour. Bot., 35, 349-375, pls. 3, figs. 133.

-, (1959): Plant microfossils from Kootenay coal-measures strata of British Columbia. Micropaleontology, 5, (3), 303-324, pls. 1-2.

- (1962): Plant microfossils from the Burrard formation of western British Columbia. Micropaleontology, 8, (2), 187-218, pls. 1-5.

SAH, S. C. D. (1953): Spores and other microremains from a carbonaceous shale (Jurassic) in andigama, Ceylon. Spolia Zeylanica, 27, (1), 1-12.

- (1955): Plant microfossils from a Jurassic shale of salt range, west Punjab (Pakistan). The Palaeobotanist, 4, 60-71.

SATo, Seiji (1961): Pollen analysis of carbonaceous matter from the Hakobuchi group in the Enbetsu district, northern Hokkaido, Japan. Palynological study on Cretaceous sediments (I). Jour. Fac. Sci., Hokkaido Univ., Ser. IV, Geol. \& Minerl., 11, (1), 77-93, pls. $1-2$.

Schemel, M. P. (1951): Small spores of the Mystic coal of Iowa. (Catalog of fossil spores and pollen, vol. 7). Am. Midland Naturalist, 46, (3), 743-750, figs. 1-4.

Schwarzbach, M. und Pflug, H. D. (1957): Beiträge zur Klimageschichte Islands VI. Das Klima des jüngeren Tertiärs in Island. N. Jb. Geol. u. Paläont., Abh. 104, (3), 279298.

ScotT, R. A. (1960): Pollen of Ephedra from the Chinle formation (upper Triassic) and the genus Equisetosporites. Micropaleontology, 6, (3), 271-276, pl. 1, text-figs. 1-2.

Sears, P. B. (1950): Pollen analyse in Old and New Mexico. Bull. Geol. Soc. Am., 61, (10), 1171.

SEWARD, A. C. (1919): Fossil plants IV.

Sitholey, R. V., Sah, S. C. D., and Dube, S. N. (1954): Plant microfossils from a carbonaceous shale (Krols) near Naini Tal. Jour. Sci. \& Indus. Research, 13, (6), 450451.

Stanley, E. A. (1961): The fossil pollen genus Aquilapollenites. Pollen et Spores, 3, (2), 329352 , pls. $1-8$. 
Stanley, E. A. and Kremp, G. O. W. (1959): Some observations on the geniculus in the pollen of Quercus prinoides. Micropalentology, 5, (3), 351-354. text-fig. 1.

TAKAHASHI, Kiyoshi (1957): Palynologisch-stratigraphische Untersuchung der tertiären Schichten im Kasuya und Fukuoka Kohlenfeld von Nordkyushu, Japan. Mem. Fac. Sci., Kyushu Univ., Ser. D, Geol., 5, (4), 199-221, Taf. 38-39, Abb. 1-6, Tab. 1-3.

- (1957): Pollen und Sporen aus den Hauptflözen im Miike und Asakura Kohlenfeld und ihre stratigraphische Bedeutung. (Jap. mit deutsch. Zusammenfassung). Jour. Mining Inst. Kyushu, 25, (12), 508-516.

- (1960): Vergleich der japanischen und mitteleuropäischen tertiären Pollen und Sporen. (Abstrakt). (jap.), Jour. Geol. Soc. Japan, 66, (778), 471.

- (1961): Pollen und Sporen des westjapanischen Alttertiärs und Miozäns. (I. Teil). Mem. Fac. Sci., Kyushu Univ., Ser. D, Geol., 11, (2), 151-255, Abb. 1-40, Tab. 1-15.

-- (1961): Pollen und Sporen des wastjapanischen Alttertiärs und Miozäns. (II. Teil). Mem. Fac. Sci., Kyushu Univ., Ser. D, Geol., 11, (3), 279-345, Taf. 13-27.

- (1962): Pollenformen aus den eozänen Kohlenflözen von Ishizuchi. Mem. Fac. Sci., Kyushu Univ., Ser. D, Geol., 12, (1), 1-26, Taf. 1-5, Abb. 1-2, Tab. 1.

- (1962): Zur Kenntnis der alttertiären Pflanzenwelt Westjapans. Jap. Jour. Geol. Geogr., 33, (2-4), 191-203, Tab. 2.

-_. (1963): Palynologisch-stratigraphische Untersuchung im Jooban-Kohlenfeld. (jap.). Sci. Rep. Fac. Sci., Kyushu Univ., Geol., 6, (2), 77-94, Taf. 9-12, Abb. 1-8, Tab. 1-6.

-_, (1963): Sporenpalaontologische Untersuchungen der Hioki-Schichtengruppe von Waku und Kiwado. Mem. Fac. Sci., Kyushu Univ., Ser. D, Geol., 14, (2), 143-157, Taf. 20-22, Abb. 1-5, Tab. 1-2.

- (1963): Pollenformen aus den Hitomaru-Schichten in der Yuya-wan Gegend. Trans. Proc. Palaeont. Soc. Japan, N. S. (51), 120-127, Taf. 18.

- (1963): Pollen und Sporen aus dem Schieferton von Ashiya, Nordkyushu. Jap. Jour. Geol. Geogr.

Thiergart, F. (1940): Die Mikropaläontologie als Pollenanalyse im Dienst der Braunkohlenforschung. Brennstoff-Geol., (13), 1-82, Taf. 1-14, Tab. 3.

- (1947): Leitpollen der untermiozänen und oberoligozänen Braunkohle und ihre systematische Stellung. Z. deutsch. Geol. Ges., 97, (1), 54-65, Taf. 1.

- (1953): Über einige Sporen und Pollen der Perutzer Schichten (Böhmen). Palaeontographica, B, 95, 53-59.

Thomson, P. W. (1949): Alttertiäre Elemente in der Pollenflora der rheinischen Braunkohle und einige stratigraphisch wichtige Pollenformen derselben. Palaeontographica, B, 90, 94-96.

Thomson, P. W. und Pflug, H. D. (1952): Die alttertiäre Braunkohle der Tongrube Zievel im Antweiler Gruben bei Satzvey/Bl. Euskirchen. N. Jb. Geol. Paläont., Abh. 96, 126.

- und - (1953): Pollen und Sporen des mitteleuropäischen Tertiärs. Palaeontographica, B, 94, 1-138, Taf. 1-15, Abb. 20, Tab. 4.

ToKUnAGa, Shigemoto (1958): Palynological study on Japanese coal II. Pollen stratigraphical investigations in the coal fields, middle Hokkaido. (in Japanese with English abstract). Report no. 181, Geol. Surv. Japan, 1-50, pls. 1-8, figs. 1-40, tab. 1-4.

Traverse, A. (1955): Pollen analysis of the Brandon lignite of Vermont. Bureau of Mines, Rept. Invest. 5151, U. S. Dept. of Interior, Washington, D. C., 1-107, pls. 8-13, text-figs. 1-7.

- (1956): Systematic methode for Mesozoic and Cenozoic plant microfossils. Micropaleontology, 2, (4), 396-398.

Ueno, J. (1952): Morphology of pollen of Metasequoia, Sciadopitys und Taiwania. Jour. Inst. Polyt. Osaka city Univ., D, Biol., 2, 23-26.

- (1959): Some palynological observations of Taxaceae, Cupressaceae and Araucariaceae. Jour. Inst Polyt. Osaka city Univ., Ser. D, 10, 75-86, Tab. 1-2, Fig. 1-4. 
USHER, J. L. (1952): Ammonite faunas of the upper Cretaceous rocks of Vancouver island, British Columbia. Canada Geol. Surv. Bull., 21, 1-119, pls. 30.

Weyland, H. und Krieger, W. (1953): Die Sporen und Pollen der Aachener Kreide und ihre Bedeutung für die Charakterisierung des mittleren Senons. Palaeontographica, B, 95, 6-29, Taf. 1-5.

WEYLAND, H. und GREIFELD, G. (1953): Über strukturbietende Blätter und pflanzliche Mikrofossilien aus den untersenonen Tonen der Gegend von Quedlinburg. Palaeontographica, B, 95, 30-52, Taf. 6-13, Abb. 4.

Weyland, H. und Pflug, H. D. (1957): Die Pflanzenreste der pliozänen Braunkohle von Ptolemais in Nordgriechenland I. Palaeontographica, B, 102, 96-109, Taf. 21-22, Abb. 1.

Weyland, H., Pflug, H. D. und JäHnichen, H. (1958): Celtoidauthus pseudorobustus n.g. n. sp., eine Ulmaceen-Blüte aus der Braunkohle der Niederlausitz. Palaeontographica, B, 105, 67-74, Taf. 11-12.

Weyland, H., Pflug, H. D. und Pantic, N. (1958): Untersuchungen über die Sporen- und Pollen-Flora einiger Jugoslawischer und Griechischer Braunkohlen. Palaeontographica, B, 105, 75-99, Taf. 13-18, Tab. 1, Kartensk. 1.

Weyland, H., Pflug, H. D. und Mueller, H. (1960): Die Pflanzenreste der pliozänen Braunkohle von Ptolemais in Nordgriechenland II. Palaeontographica, B, 106, 71-98, Taf. 8-14, Abb. 1, Diagr. 3.

WEYLAND, H. und TAKAHASHI, K. (1961): Pflanzenreste aus der Braunkohlengrube „Herman“ bei Heerlen, Holländisch Limburg. Palaeontographica, B, 109, 93-107, Taf. 42-44, Tab. 1.

WICHER, C. A. (1934): Sporenformen der Flammkohle des Ruhrgebietes. Systematische Untersuchung eines Flammkohlenpartie (Westfal. C) auf seinen Sporeninhalt und kritischer Ausblick auf den Wert der Sporen für die Stratigraphie des Karbons. (Catalog of fossil spores and pollen, vol. 5). Arb. Inst. Paläont. u. Petrogr. Brennst., Preuss. Geol. L-A., 4, 165-212, Taf. 8, Abb. 3.

Wilson, L. R. and COE, E. A. (1940): Descriptions of some unassigned plant microfossils from the Des Moines series of Iowa. Am. Midland Naturalist, 23, (1), 182-186, pl. 1.

Wilson, L. R. and Webster, R. M. (1946): Plant microfossils from a Fort Union coal of Montana. Am. Jour. Botany, 33, (4), 271-278.

Wodehouse, R. P. (1933): Tertiary pollen-II. The oil shales of the Eocene Green River formation. (Catalog of fossil spores and pollen, vol. 1). Bull. Torr. Bot. Club, 60, 479-524, text-figs. 56 .

- (1935): Pollen grains. 574, Mcgraw-Hill book Co. New York and London.

WolfF, H. (1934): Mikrofossilien des pliozänen Humodils der Grube Freigericht bei Dettingen a. M. und Vergleich mit älteren Schichten des Tertiärs so wie posttertiären Ablagerungen. (Catalog of fossil spores and pollen, vol. 4). Arb. Inst. Palaeobot. u. Petrogr. Brennst. Preuss. Geol. L.-A., 5, 55-86, Taf. 5, Abb. 3.

Yamasaki, Tsugio (1933): Die Morphologie der Pollen und Sporen. (jap.). Mitteil. Versuchspflanz. Univ. Kyoto (Enshurin-Hokoku), 5, 1-27, Taf. 1-59.

ZAKLINSKAJA, E. D. (1957): Stratigraphic significance of pollen grains of gymnosperms of the Cenozoic deposits of the Irtysh basin and of the northern Aral basin. (Catalog of fossil spores and pollen, vol. 4). Acad. Sci. USSR, works of Geol. Inst. Contr. 6, 1--184, pls. 17. 
Kiyoshi TaKahashi

Sporen und Pollen der oberkretazeischen Hakobuchi-

Schichtengruppe, Hokkaido

Tafeln 23-44 
Tafel 23 


\section{Erklärung zu Tafel 23}

(Untere Hakobuchi-Schichtengruppe von Hatsune-Sawa; ca. 833 fach vergr.)

Fig. 1-3. Cicatricosisporites cf. dorogensis R. Potoní \& Gelletich

Fig. 1: Präparat GK-V 3131; Fig. 2: Präparat GK-V 3143; Fig. 3: Präparat GK-V 3133.

Fig. 4-17. Gleicheniidites marginatus n. sp.

Fig. 4, 8, 10, 13, 14: Präparat GK-V 3141; Fig. 7: Präparat GK-V 3142, Holotypus; Fig. 12, 15: Präparat GK-V 3142; Fig. 6, 11: Präparat GK-V 3143; Fig. 5, 9, 16, 17: Präparat GK-V 3161.

Fig. 18,19. Concavisporites macellus $\mathrm{n}$. sp.

Fig. 18: Präparat GK-V 3131, Holotypus; Fig. 19: Präparat GK-V 3131.

Fig. 20. Concavisporites sp. a

Präparat GK-V 3181.

Fig. 21a, b. ? Divisisporites sp.

Präparat GK-V 3131.

Fig. 22-24. Verrucosisporites permirus n. sp.

Fig. 22: Präparat GK-V 3172, Holotypus; Fig. 23a, b: Präparat GK-V 3172; Fig. 24: Präparat GK-V 3182. 

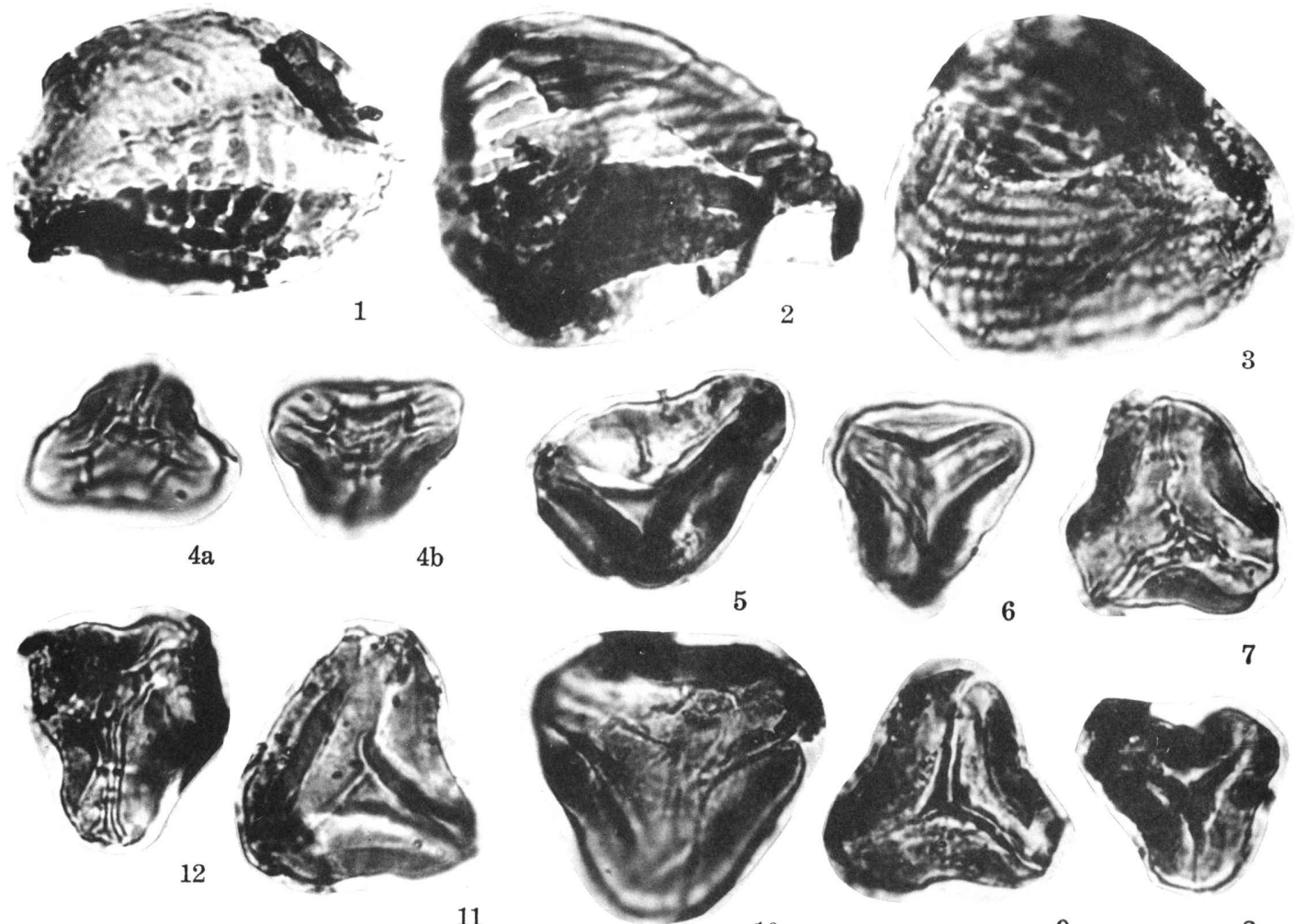

10

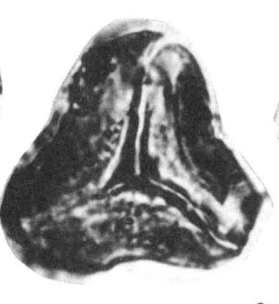

7
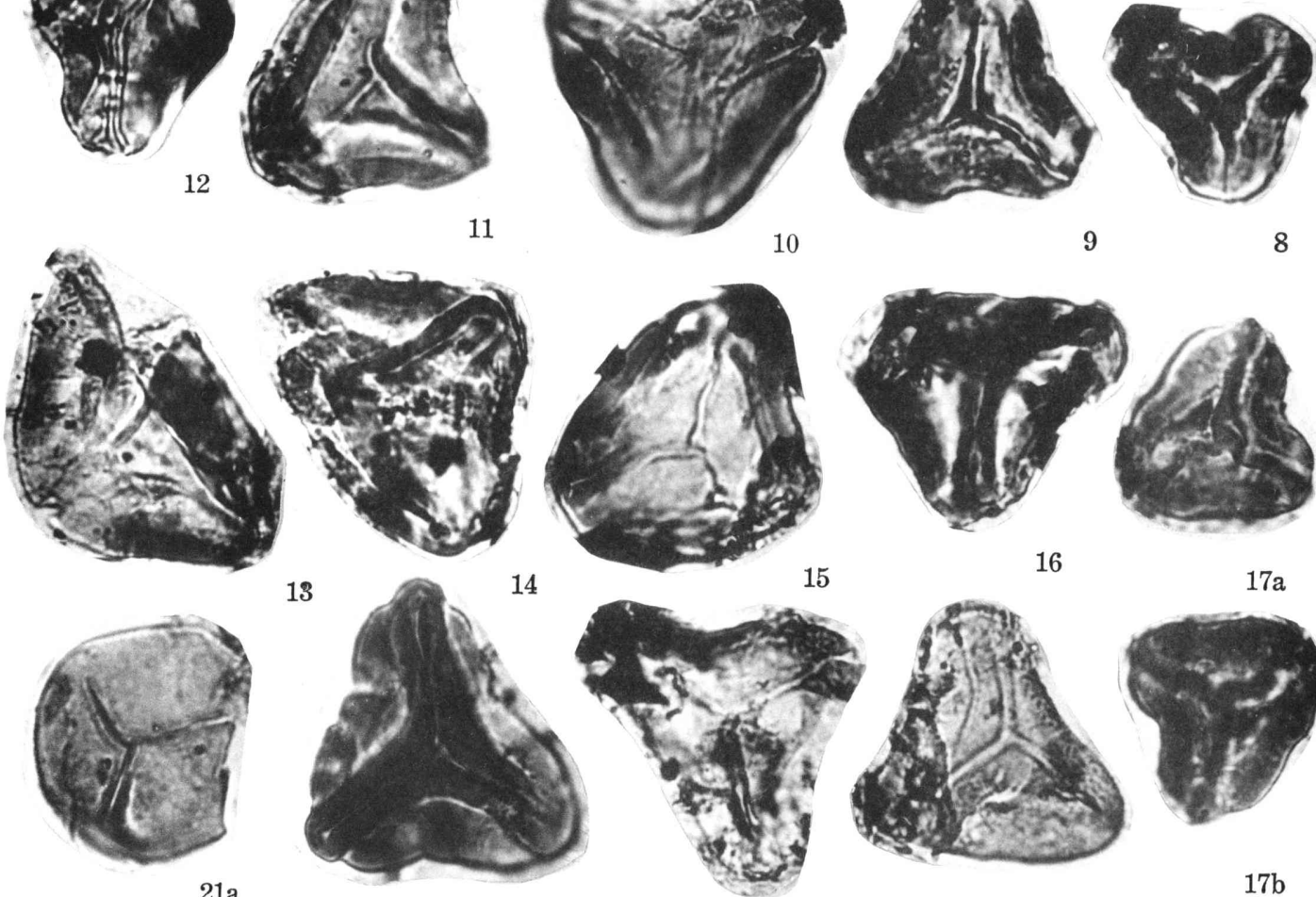

9

$21 a$

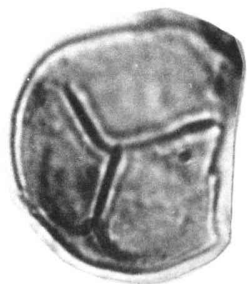

$21 b$
20

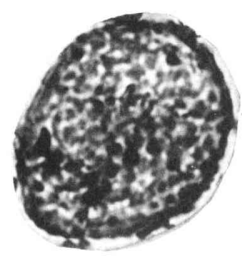

22

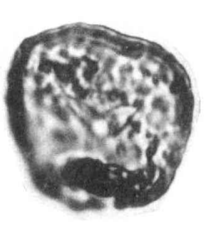

$23 a$
19

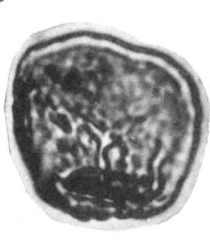

$23 \mathrm{~b}$
16

$17 a$
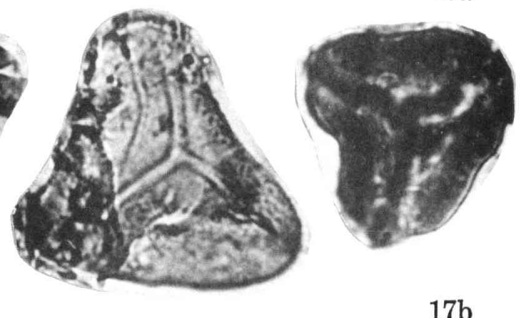

$17 \mathrm{~b}$

K. TAkahashi: Sporen und Pollen der Hakobuchi-Schichtengruppe. 


\section{Tafel 24}




\section{Erklärung zu Tafel 24}

(Untere Hakobuchi-Schichtengruppe von Hatsune-Sawa; ca. 833 fach vergr.)

Fig. 1-12. Stereisporites limbatus n. sp.

Fig. 5, 7, 8: Präparat GK-V 3131; Fig. 4a, b: Präparat GK-V 3132, Holotypus; Fig. 11: Präparat GK-V 3132; Fig. 1: Präparat GK-V 3133; Fig. 3, 6, 10: Präparat GK-V 3141; Fig. 2: Präparat GK-V 3143; Fig. 9: Präparat GK-V 3154; Fig. 12: Präparat GK-V 3171.

Fig. 13-15. Stereisporites grossus n. sp.

Fig. 13, 14: Präparat GK-V 3172; Fig. 13: Holotypus; Fig. 15: Präparat GK-V 3181.

Fig. 16-23. Stereisporites pseudostereoides n. sp.

Fig. 17, 22: Präparat GK-V 3131; Fig. 16, 19, 20: Präparat GK-V 3132;

Fig. 16: Holotypus; Fig. 21: Präparat GK-V 3133; Fig. 18: Präparat GK-V 3161; Fig. 23: Präparat 3172.

Fig. 24,25. Stereisporites ? tenuiculus n. sp.

Fig. 24: Präparat GK-V 3131; Fig. 25: Holotypus, Präparat GK-V 3132. 

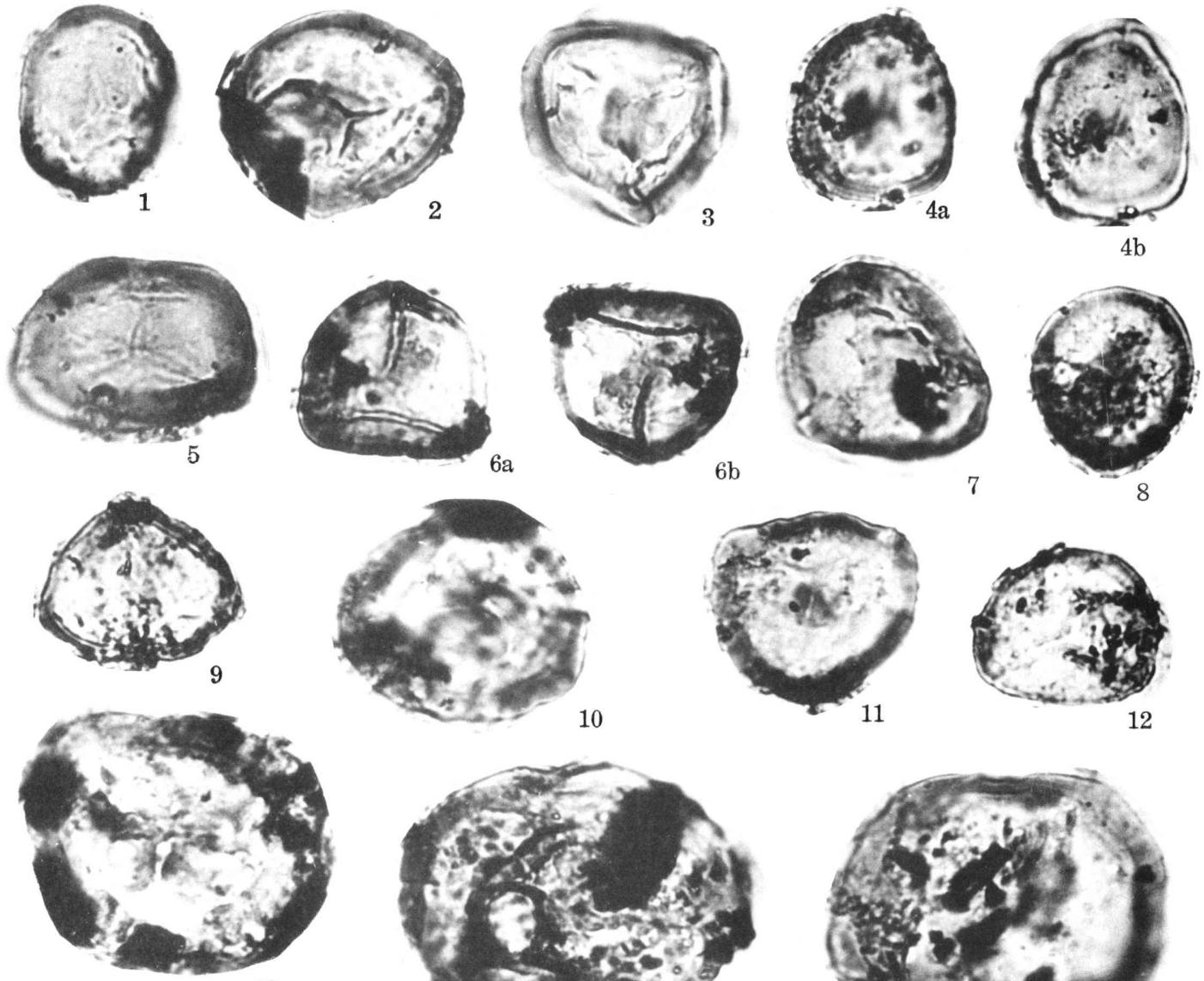

10
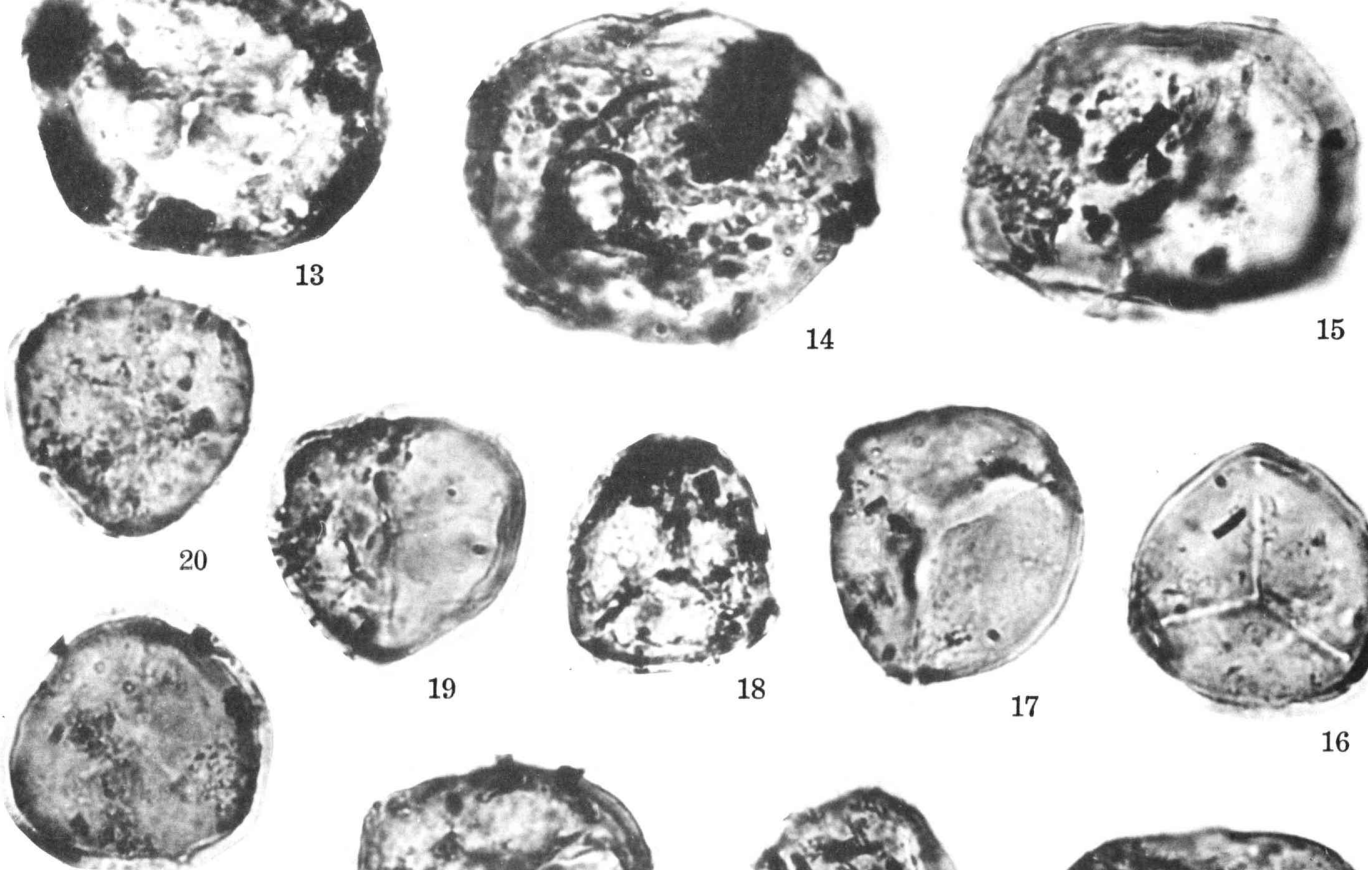

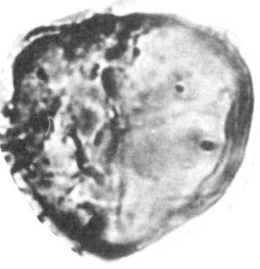

19

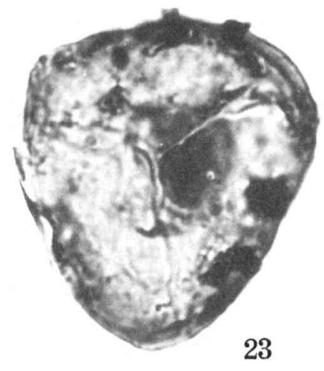

18

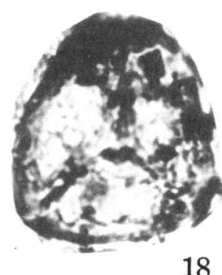

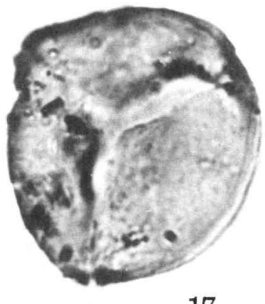

17

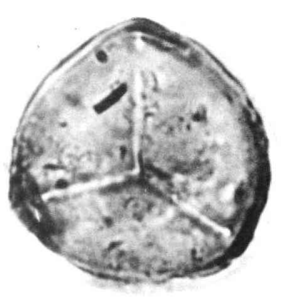

16

21
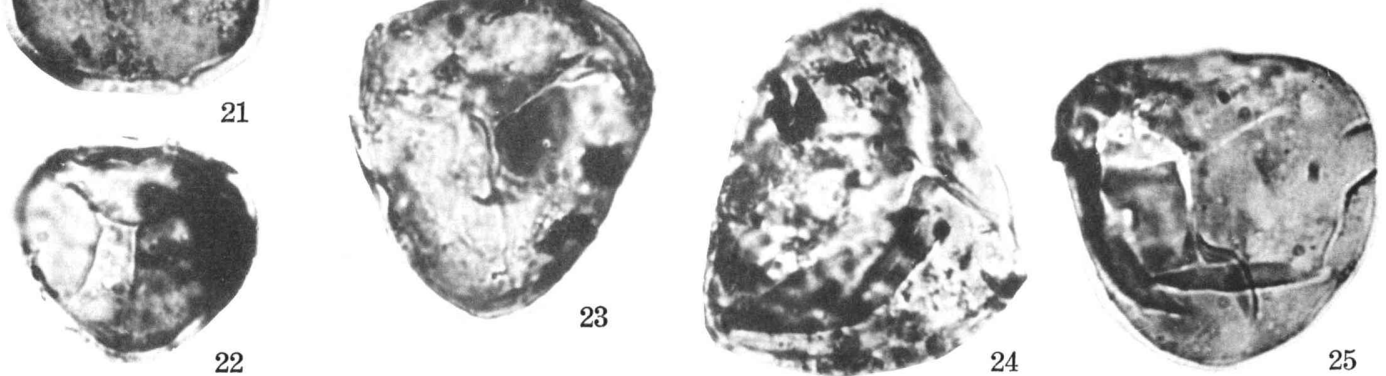

K. Takahashi: Sporen und Pollen der Hakobuchi-Schichtengruppe. 
Tafel 25 


\section{Erklärung zu Tafel 25}

(Untere Hakobuchi-Schichtengruppe von Hatsune-Sawa; ca. 833 fach vergr.)

Fig. 1-4. Laevigatisporites hokkaidoensis n. sp.

Fig. 1: Präparat GK-V 3142; Fig. 2: Präparat GK-V 3143, Holotypus;

Fig. 3: Präparat GK-V 3153; Fig. 4: Präparat GK-V 3141.

Fig. 5, 6. Laevigatisporites magnus n. sp.

Fig. 5a, b: Präparat GK-V 3133, Holotypus; Fig. 6: Präparat GK-V 3141.

Fig. 7-15. Punctatisporites punctulatus n. sp.

Fig. 7: Präparat GK-V 3131, Holotypus; Fig. 8-10: Präparat GK-V 3132; Fig. 11, 12: Präparat GK-V 3141; Fig. 13: Präparat GK-V 3142;

Fig. 14: Präparat GK-V 3143; Fig. 15: Präparat GK-V 3161.

Fig. 16,17. Punctatisporites hatsunesawaensis $\mathrm{n}$. sp.

Fig. 16: Präparat GK-V 3141; Fig. 17: Präparat GK-V 3142, Holotypus.

Fig. 18,19. Dictyophyllidites divergens (SATO) n. comb.

Fig. 18: Präparat GK-V 3132; Fig. 19: Präparat GK-V 3133. 

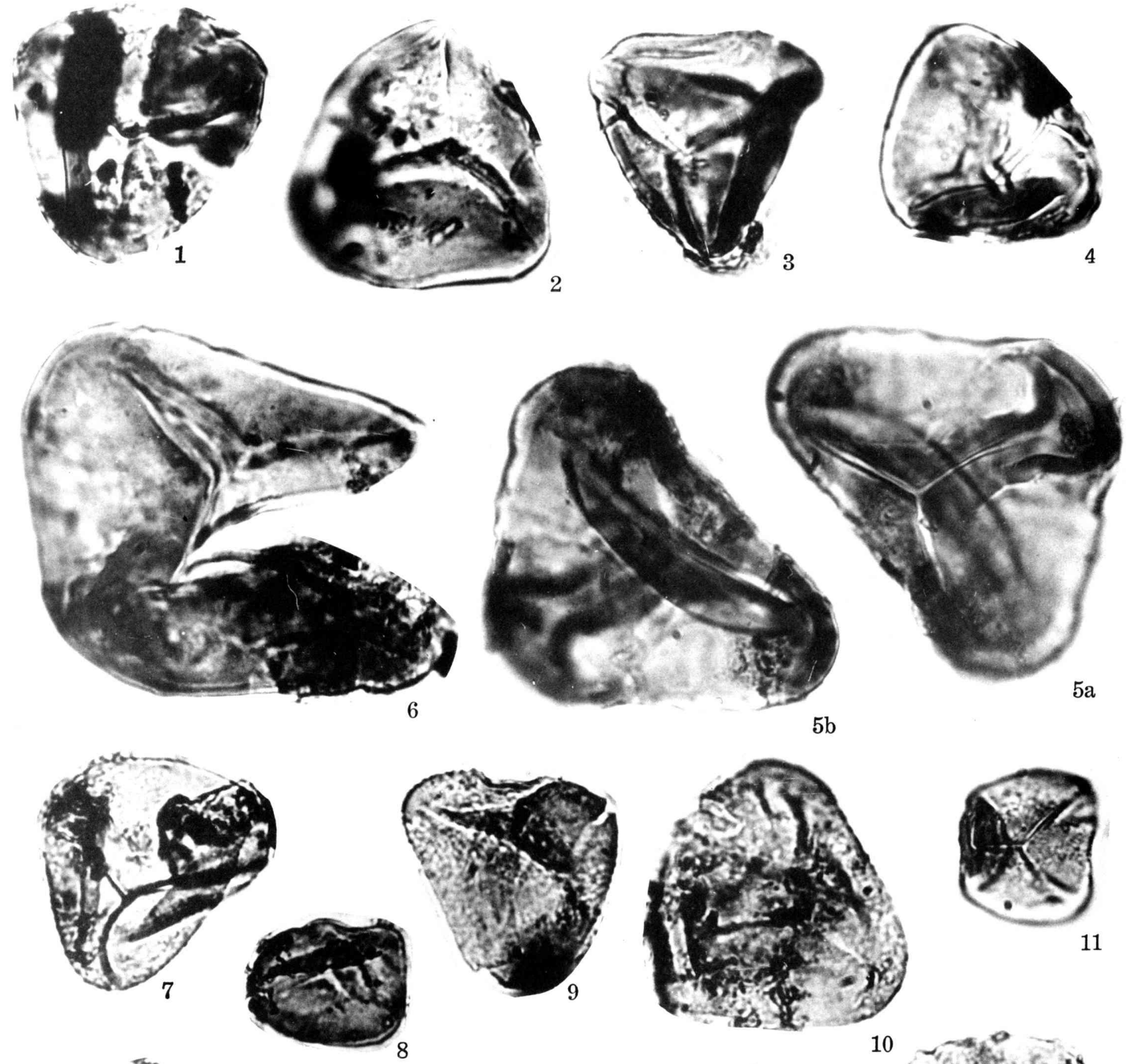

$5 \mathrm{~b}$
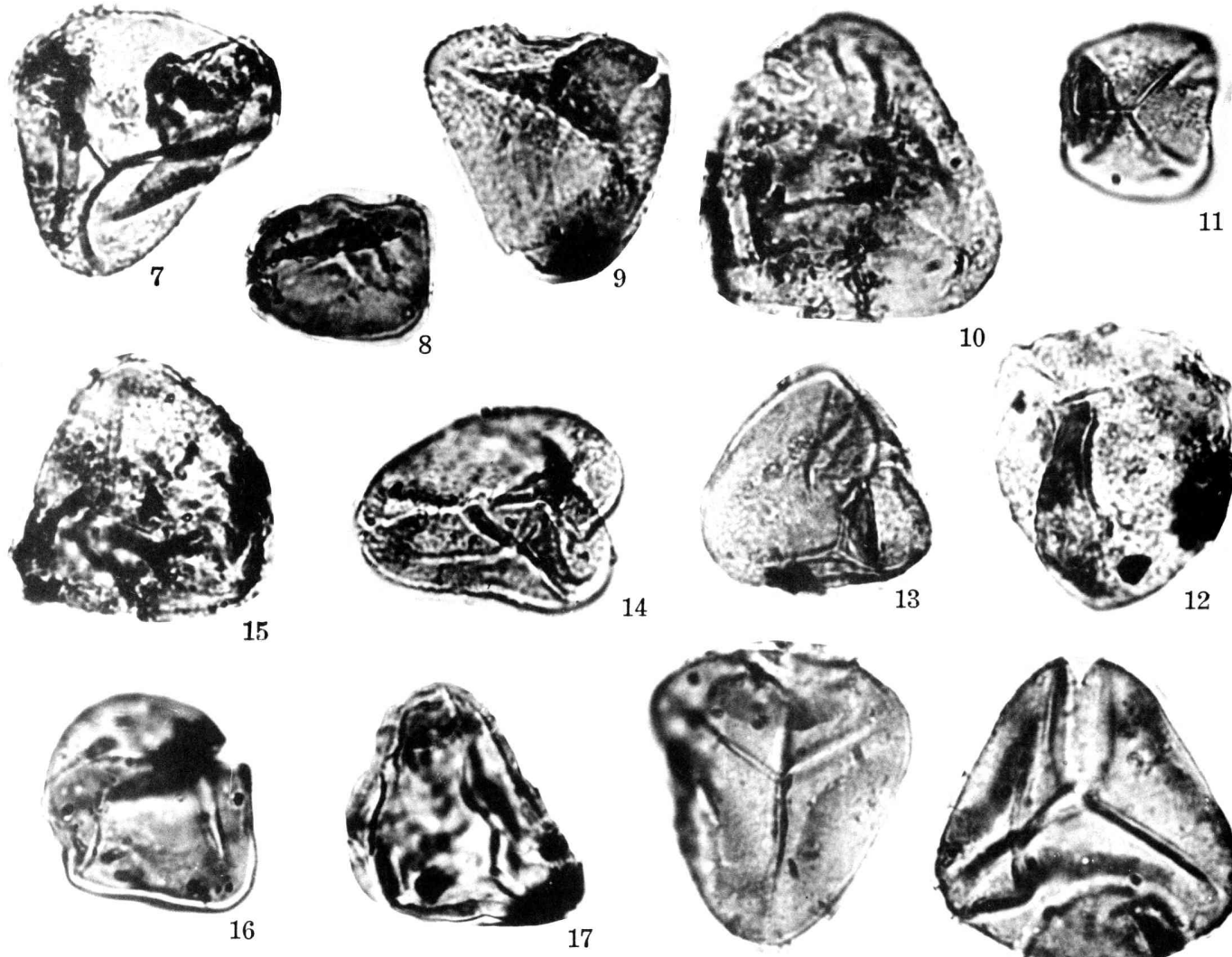

18
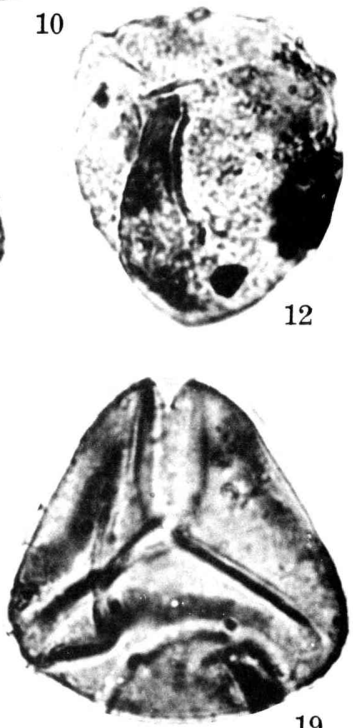

K. Takahashi: Sporen und Pollen der Hakobuchi-Schichtengruppe. 
Tafel 26 


\section{Erklärung zu Tafel 26}

(Untere Hakobuchi-Schichtengruppe von Hatsune-Sawa; ca. 833 fach vergr.)

Fig. 1. Punctatisporites sp.

Präparat GK-V 3142.

Fig. 2a, b. Corrugatisporites sp.

Präparat GK-V 3133.

Fig. 3-5. Rugulatisporites salebrosus $\mathrm{n}$. sp.

Fig. 3a-b, 5a-b: Präparat GK-V 3132; Fig. 4a-b: Präparat GK-V 3133, Holotypus.

Fig. 6-8. Rugulatisporites parvirugulatus n. sp.

Fig. 6: Präparat GK-V 3143, Holotypus; Fig. 7, 8: Präparat GK-V 3142 .

Fig. 9. Biretisporites ? minus n. sp.

Präparat GK-V 3181, Holotypus.

Fig. 10. ? Microreticulatisporites sp.

Präparat GK-V 3181.

Fig. 11. Microreticulatisporites sp.

Präparat GK-V 3154. 

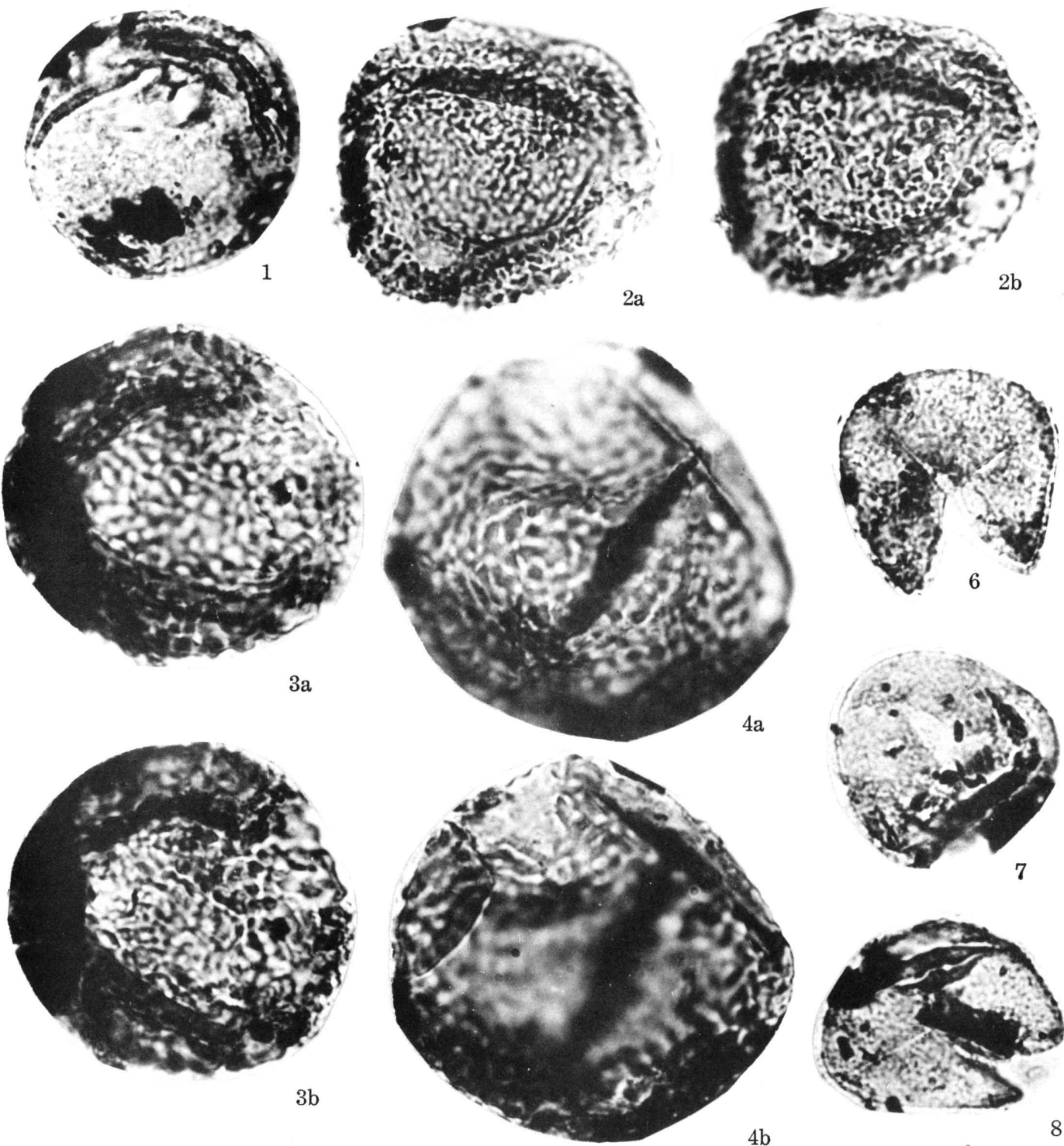

$3 b$
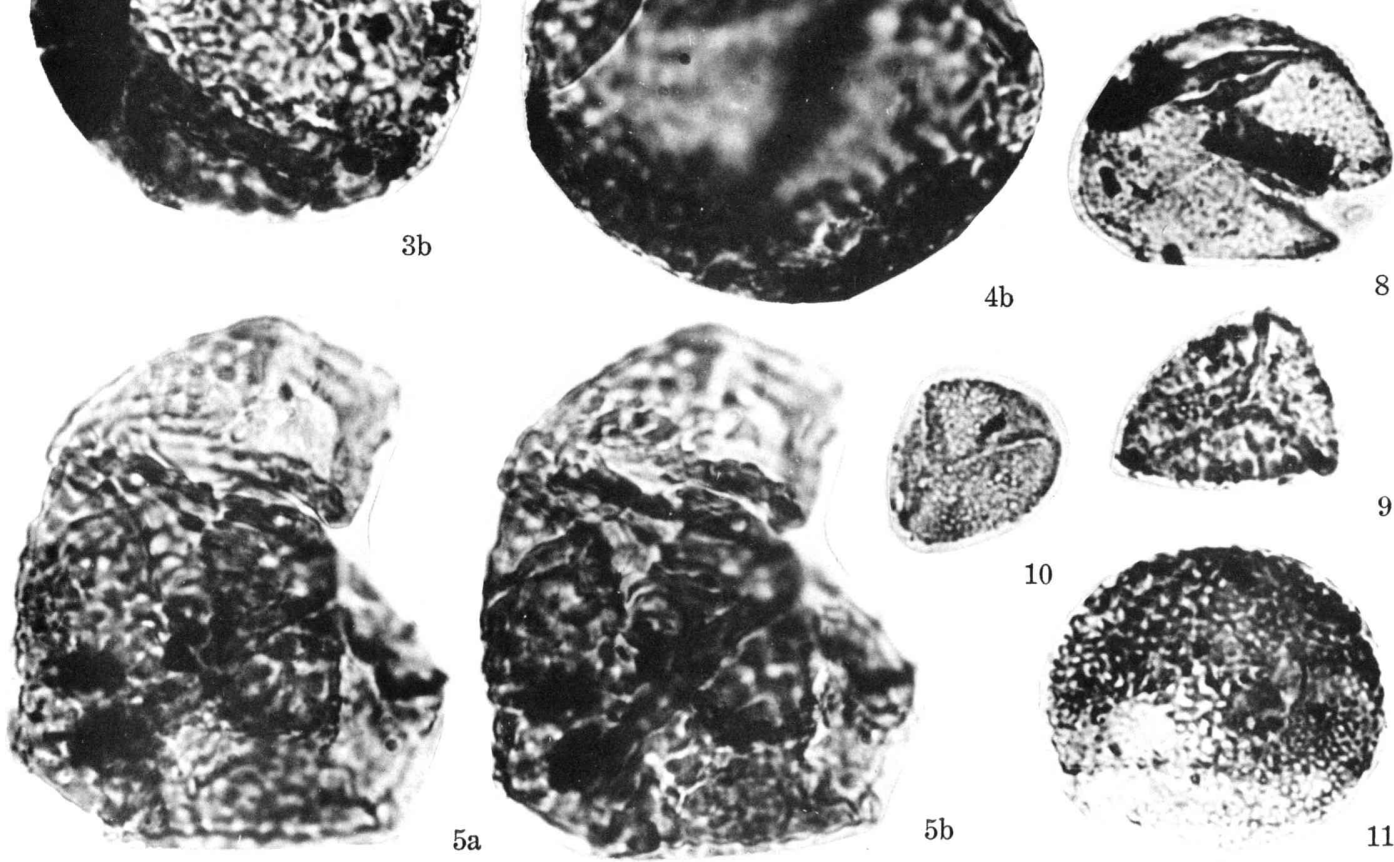

K. Takahashi : Sporen und Pollen der Hakobuchi-Schichtengruppe. 
Tafel 27 


\section{Erklärung zu Tafel 27}

(Untere Hakobuchi-Schichtengruppe von Hatsune-Sawa; ca. 833 fach vergr.)

Fig. 1. Punctatisporites punctulatus n. sp.

Präparat GK-V 3131.

Fig. 2-9. Apiculatisporis inouei n. sp.

Fig. 2, 8: Präparat GK-V 3131; Fig. 3, 4: Präparat GK-V 3132; Fig. 9: Präparat GK-V 3133; Fig. 5: Präparat GK-V 3141; Fig. 6: Präparat GK-V 3152, Holotypus; Fig. 7: Präparat GK-V 3173.

Fig. 10-15. Baculatisporites papillosus n. sp.

Fig. 13: Präparat GK-V 3131; Fig. 12, 15: Präparat GK-V 3132; Fig. 12: Holotypus; Fig. 10: Präparat GK-V 3141; Fig. 11: Präparat GKV 3143; Fig. 14: Präparat GK-V 3153. 

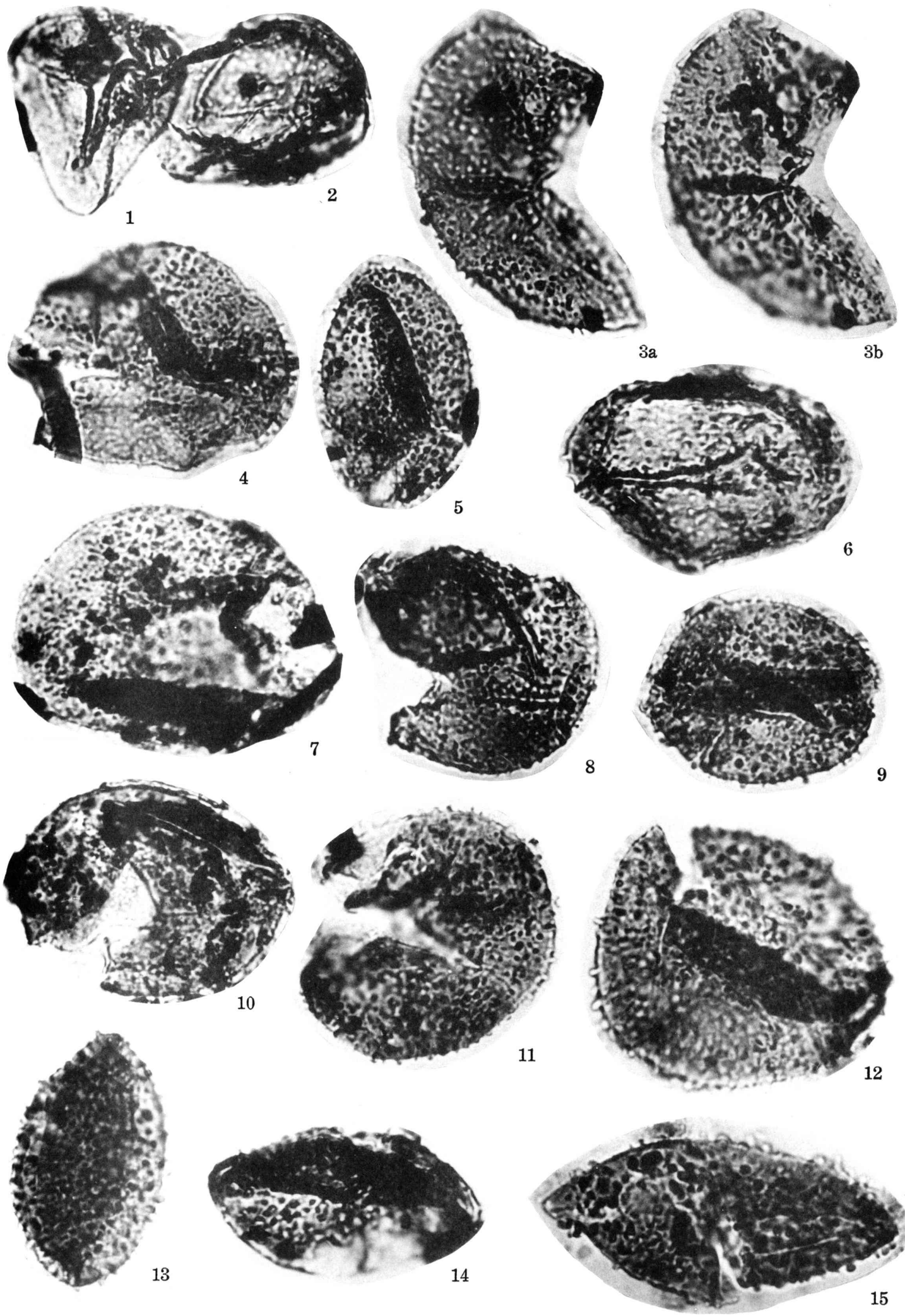

K. Takahashi : Sporen und Pollen der Hakobuchi-Schichtengruppe. 


\section{Tafel 28}




\section{Erklärung zu Tafel 28}

(Untere Hakobuchi-Schichtengruppe von Hatsune-Sawa; ca. 833 fach vergr.)

Fig. 1-3. Baculatisporites papillosus n. sp.

Fig. 1: Präparat GK-V 3172; Fig. 2: Präparat GK-V 3154; Fig. 3?: Präparat GK-V 3142.

Fig. 4, 5. Apiculatisporis micracanthus n. sp.

Fig. 4a, b: Präparat GK-V 3155, Holotypus: Fig. 5: Präparat KG-V 3161 .

Fig. 6-11. Baculatisporites validus n. sp.

Fig. 6: Präparat GK-V 3155; Fig. 7: Präparat GK-V 3131; Fig. 8, 9: Präparat GK-V 3141; Fig. 9: Präparat GK-V 3143, Holotypus; Fig. 10: Präparat GK-V 3171.

Fig. 12-14. Triplanosporites sinuatus n. sp.

Fig. 12, 13: Präparat GK-V 3181; Fig. 14: Präparat GK-V 3161, Holotypus. 

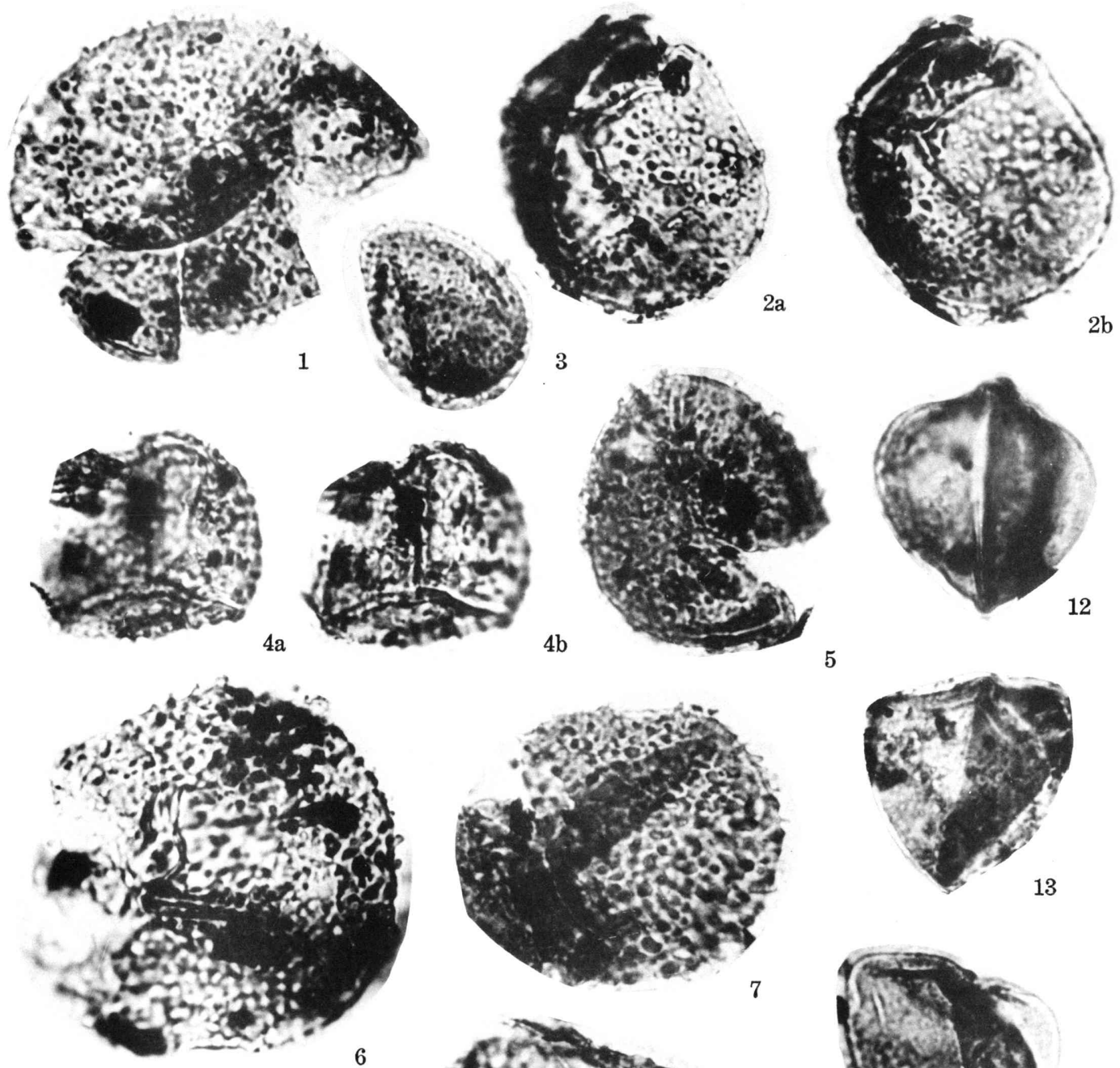

5


9
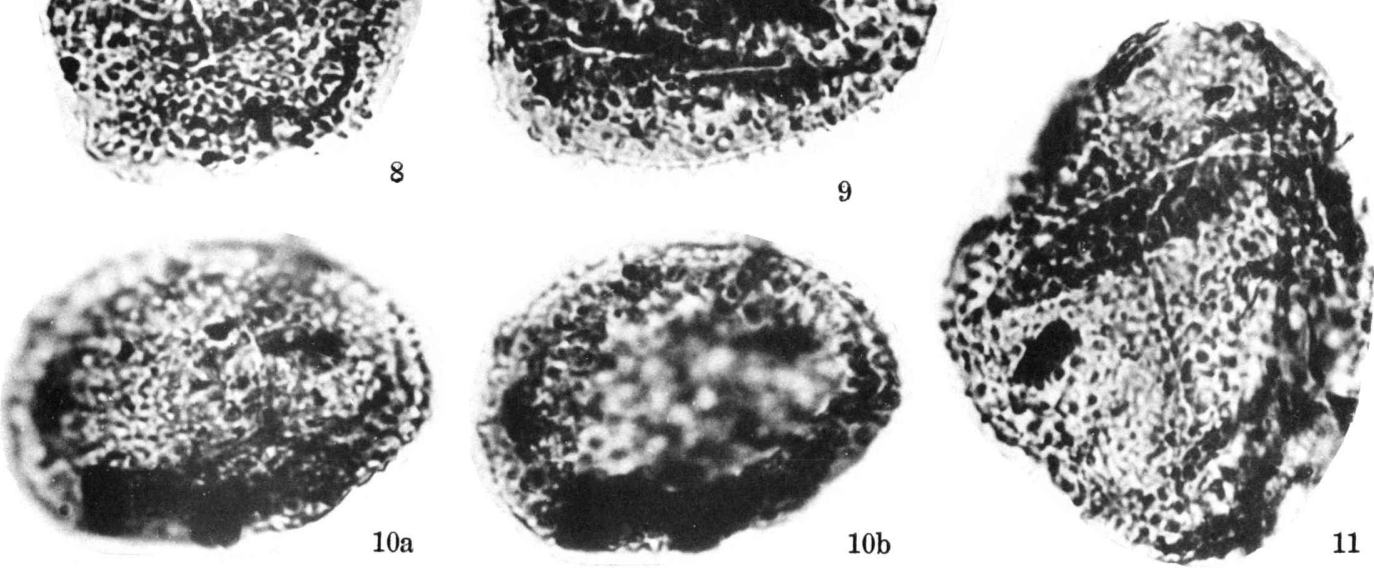

K. Takahashi: Sporen und Pollen der Hakobuchi-Schichtengruppe. 
Tafel 29 


\section{Erklärung zu Tafel 29}

(Untere Hakobuchi-Schichtengruppe von Hatsune-Sawa; ca. 833 fach vergr.)

Fig. 1-7. Laevigatosporites prominens $\mathrm{n}$. sp.

Fig. 1-3: Präparat GK-V 3131; Fig. 4: Präparat GK-V 3142; Fig. 5:

Präparat GK-V 3141, Holotypus; Fig. 6, 7: Präparat GK-V 3161.

Fig. 8. Laevigatosporites probatus $\mathrm{n}$. sp.

Präparat GK-V 3131, Holotypus.

Fig. 8-13. Laevigatosporites dehiscens TAKAHASHI

Fig. 9: Präparat GK-V 3132; Fig. 10, 11: Präparat GK-V 3131; Fig. 12: Präparat GK-V 3153; Fig. 13: Präparat GK-V 3154.

Fig. 14-19. Laevigatosporites senonicus $\mathrm{n}$. sp.

Fig. 14, 15: Präparat GK-V 3141; Fig. 14: Holotypus; Fig. 16: Präparat GK-V 3142; Fig. 17: Präparat GK-V 3153; Fig. 18: Präparat GK-V 3161; Fig. 19: Präparat GK-V 3181.

Fig. 20. Laevigatosporites ovatus WILSON \& WEBSER

Präparat GK-V 3161.

Fig. 21. Laevigatosporites sp.

Präparat GK-V 3161. 

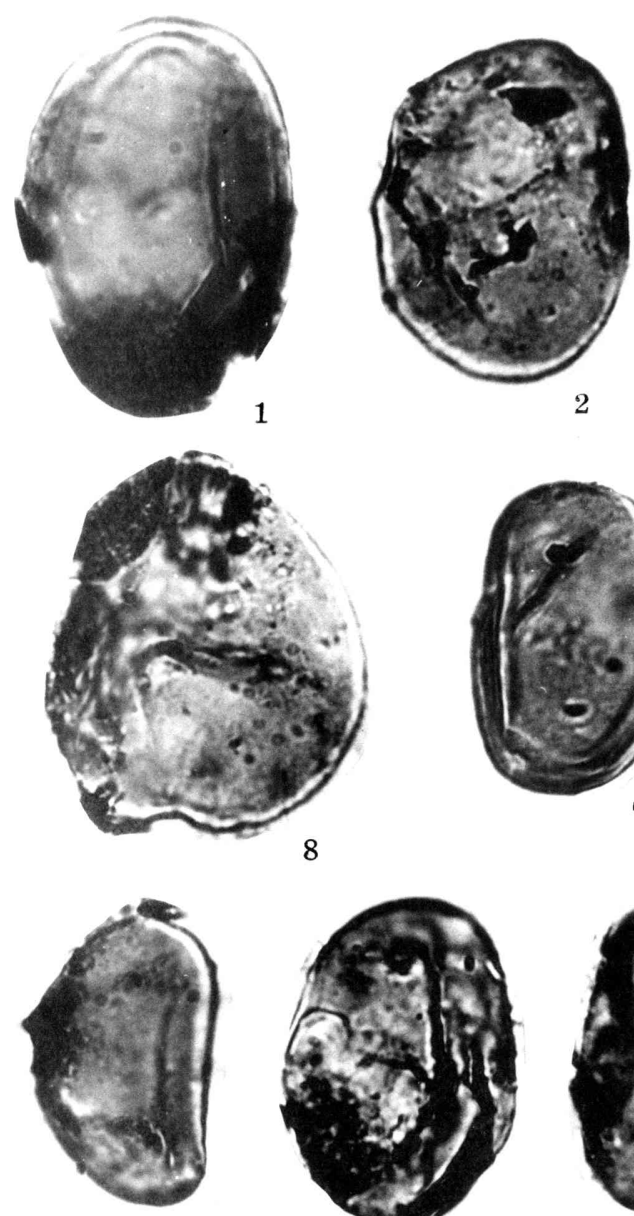

9

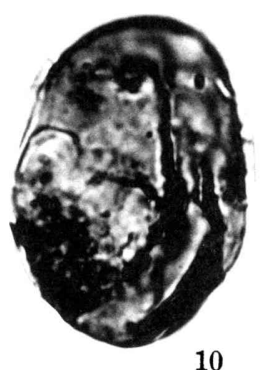

10

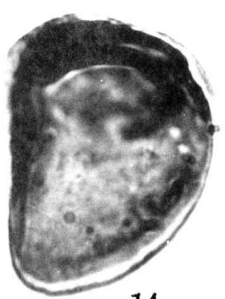

14
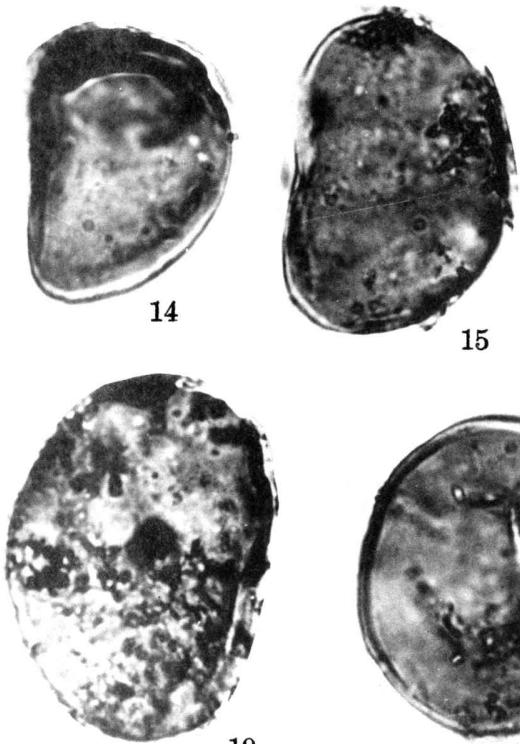

19

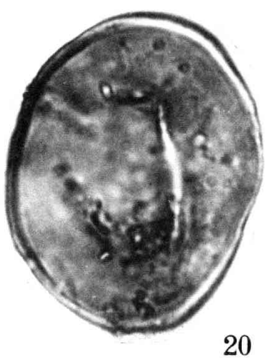

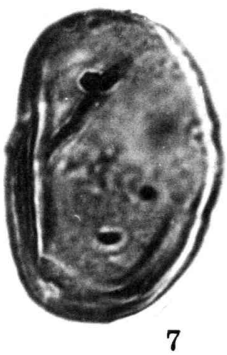

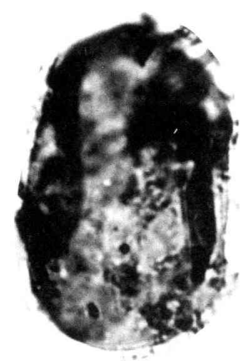

11

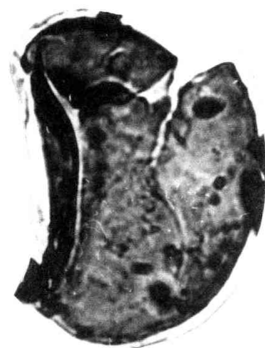

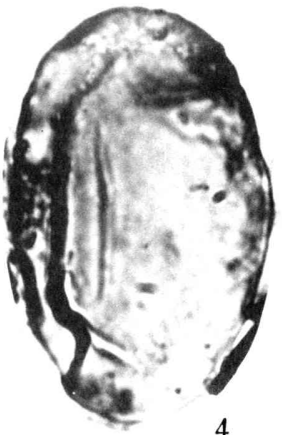
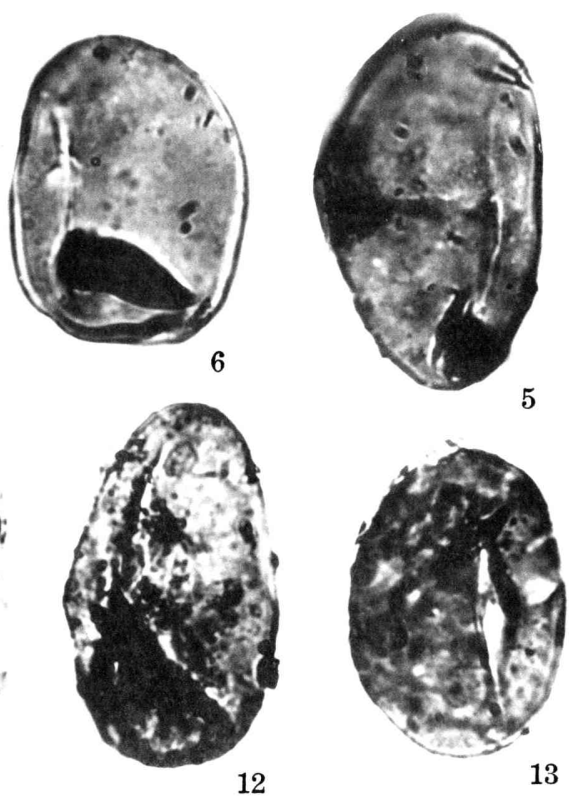

13

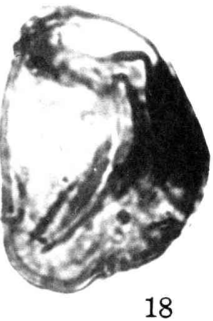

17

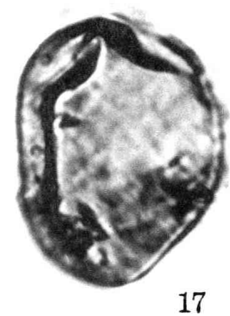

17

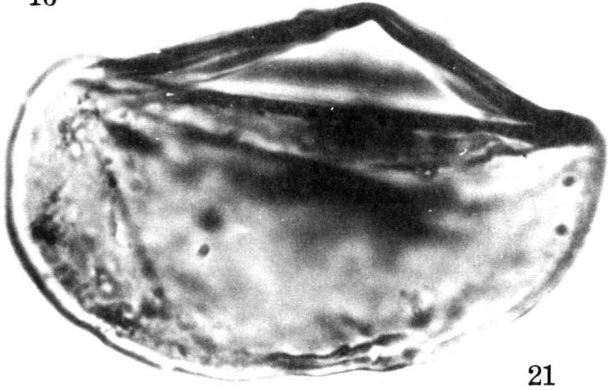

K. Takahashi : Sporen und Pollen der Hakobuchi-Schichtengruppe. 
Tafel 30 
Erklärung zu Tafel 30

(Untere Hakobuchi-Schichtengruppe von Hatsune-Sawa; ca. 833 fach vergr.)

Fig. 1-11. Polypodiisporites repandus $\mathrm{n}$. sp.

Fig. 1, 2, 8, 10: Präparat GK-V 3131; Fig. 3, 4: Präparat GK-V 3132;

Fig. 5a, b: Präparat GK-V 3133, Holotypus; Fig. 6, 9: Präparat GK-V

3154; Fig. 7: Präparat GK-V 3143; Fig. 11: Präparat GK-V 3152. 


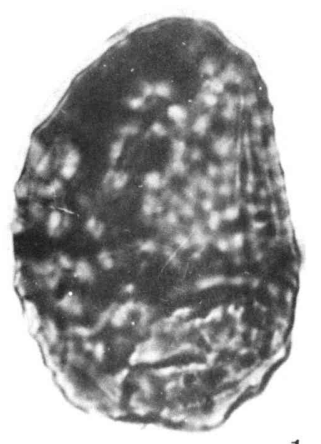

$1 \mathrm{a}$

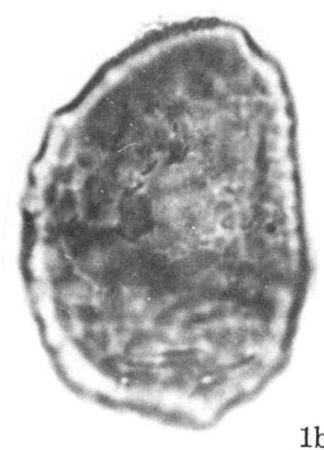

$1 b$
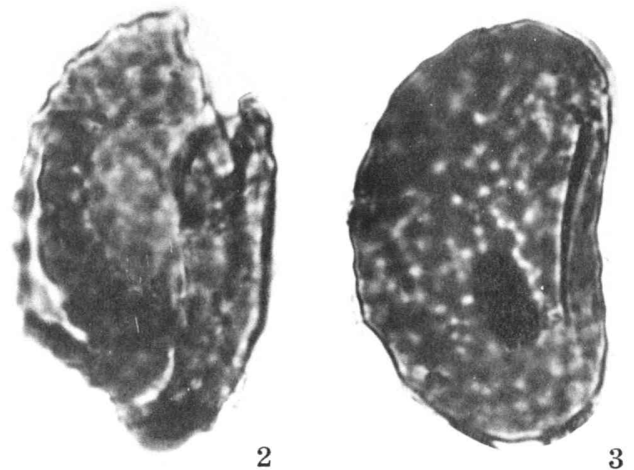

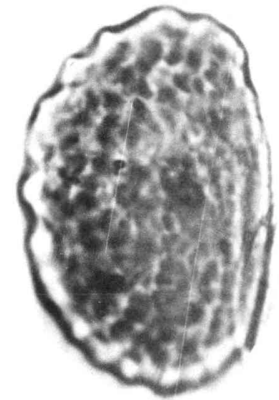

$5 a$
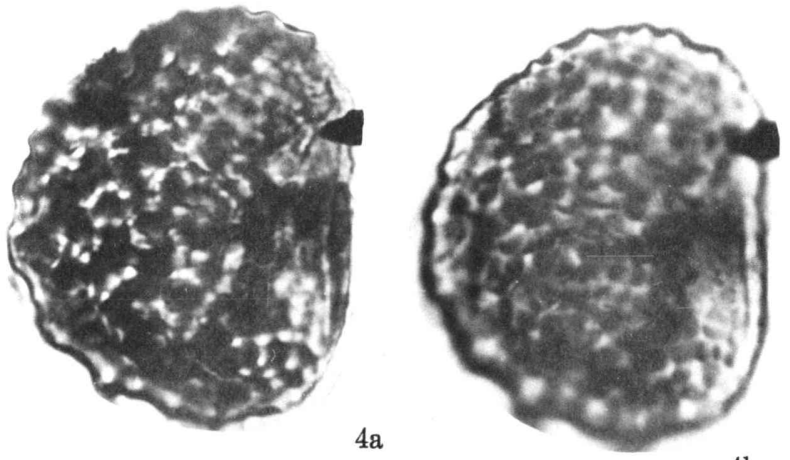

$4 \mathrm{~b}$
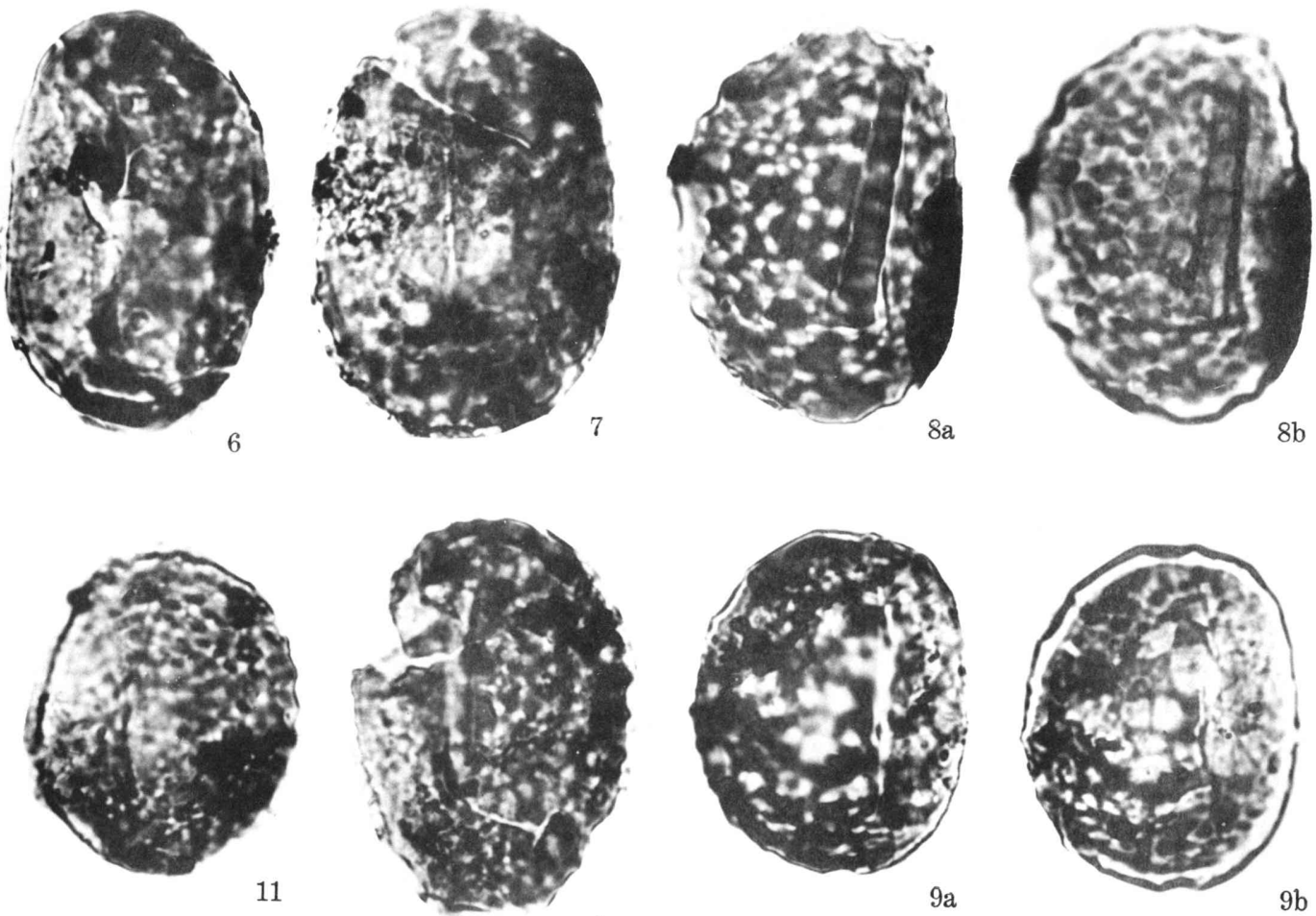

10

K. TAkahashi: Sporen und Pollen der Hakobuchi-Schichtengruppe. 
Tafel 31 


\section{Erklärung zu Tafel 31}

(Untere Hakobuchi-Schichtengruppe von Hatsune-Sawa; ca. 833 fach vergr.)

Fig. 1. Polypodiisporites repandus n. sp.

Präparat GK-V 3131.

Fig. 2. Polypodiisporites invisus n. sp.

Präparat GK-V 3152, Holotypus.

Fig. 3. Verrucatosporites sp. a

Präparat GK-V 3131.

Fig. 4, 5. Undulatosporites rugulatus n. sp.

Fig. 4: Präparat GK-V 3173, Holotypus; Fig. 5: Präparat GK-V 3172.

Fig. 6-9. Cicatricososporites ? ellipsoideus n. sp.

Fig. 6: Präparat GK-V 3153; Fig. 7: Präparat GK-V 3143, Holotypus;

Fig. 8: Präparat GK-V 3181; Fig. 9: Präparat GK-V 3171.

Fig. 10. ? Cicatricososporites sp.

Präparat GK-V 3131.

Fig. 11a, b. Schizosporis sp.

Präparat GK-V 3172.

Fig. 12. Inaperturopollenites magnus (R. РотоNIE) Thomson \& PFLUG

Präparat GK-V 3132. 
Mem. Fac. Sci., Kyushu Univ., Ser. D, Geology, Vol. XIV

Tafel 31
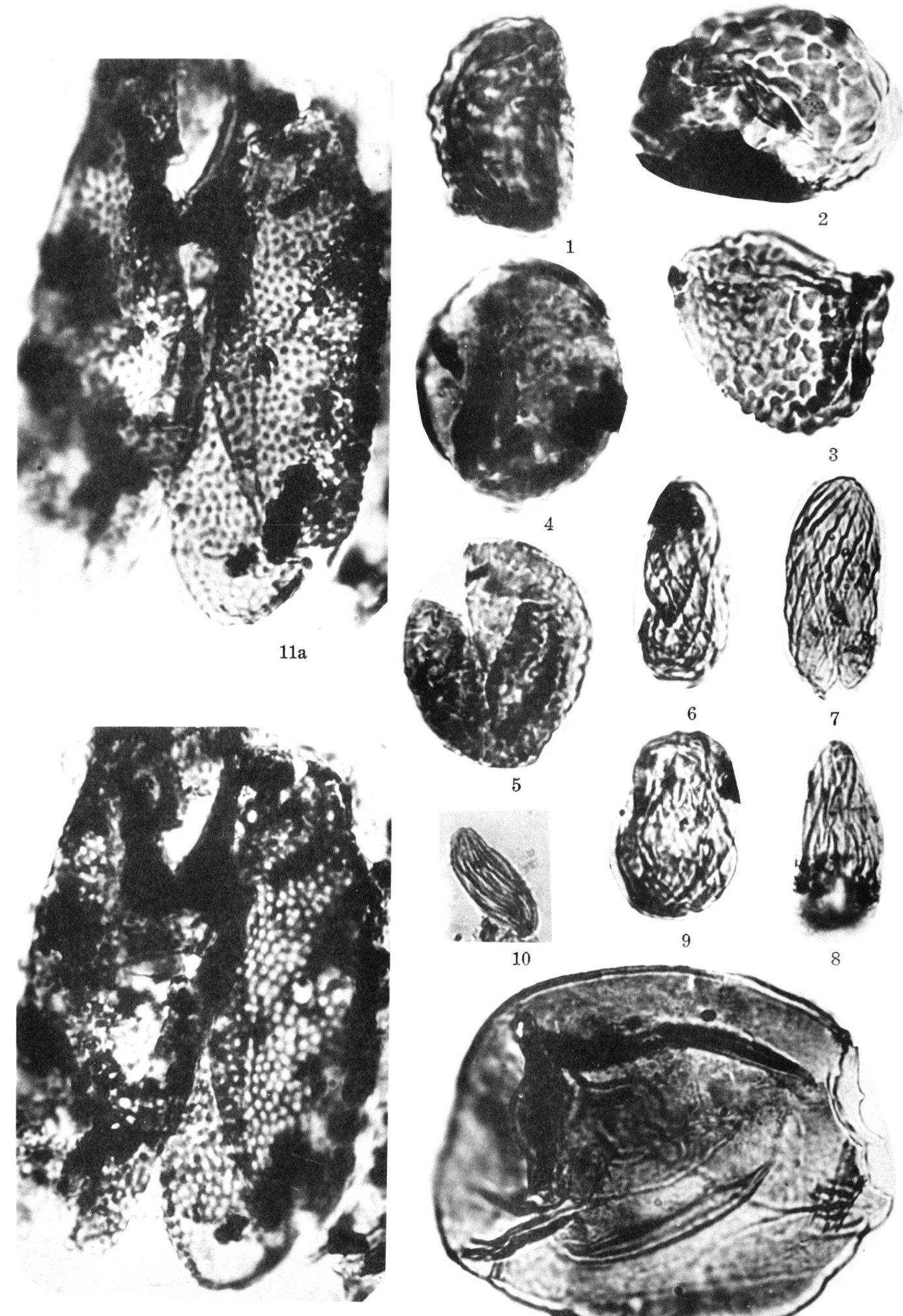

$11 b$

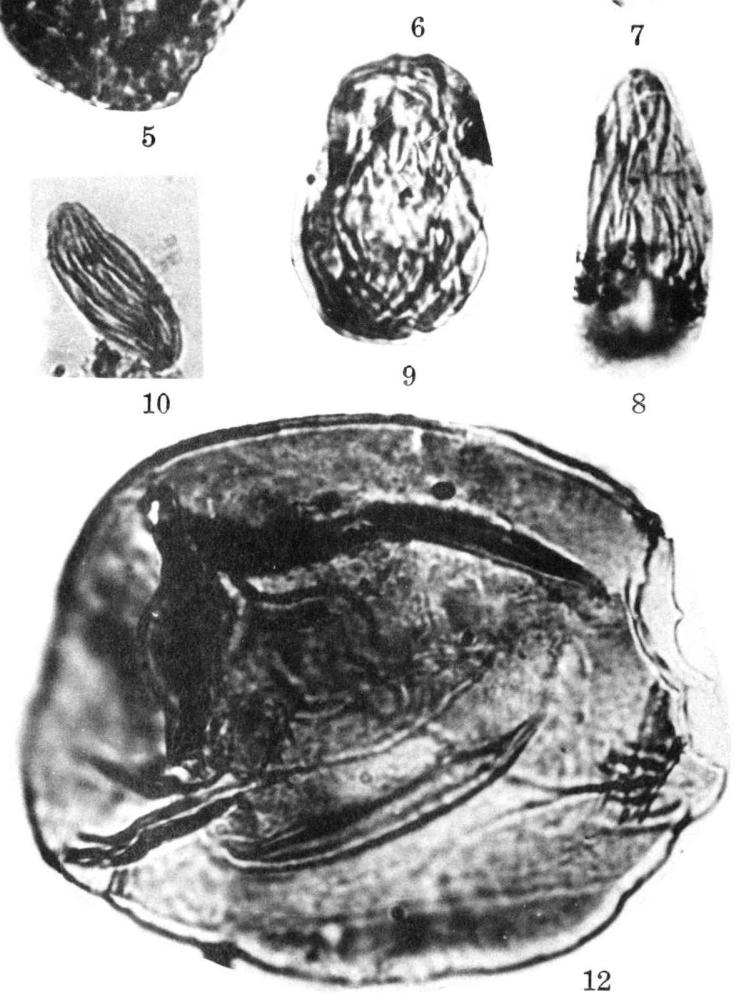

K. Takahashi: Sporen und Pollen der Hakobuchi-Schichtengruppe. 


\section{Tafel 32}




\section{Erklärung zu Tafel 32}

(Untere Hakobuchi-Schichtengruppe von Hatsune-Sawa; ca. 833 fach vergr.)

Fig. 1-17. Weylandipollis retiformis n. gen., n. sp.

Fig. 1, 11, 12, 16: Präparat GK-V 3132; Fig. 2, 4: Präparat GK-V 3143;

Fig. 2a, b: Holotypus; Fig. 3, 5, 8-10, 13-15: Präparat GK-V 3131; Fig.

4, 6: Präparat GK-V 3141; Fig. 17: Präparat GK-V 3133. 

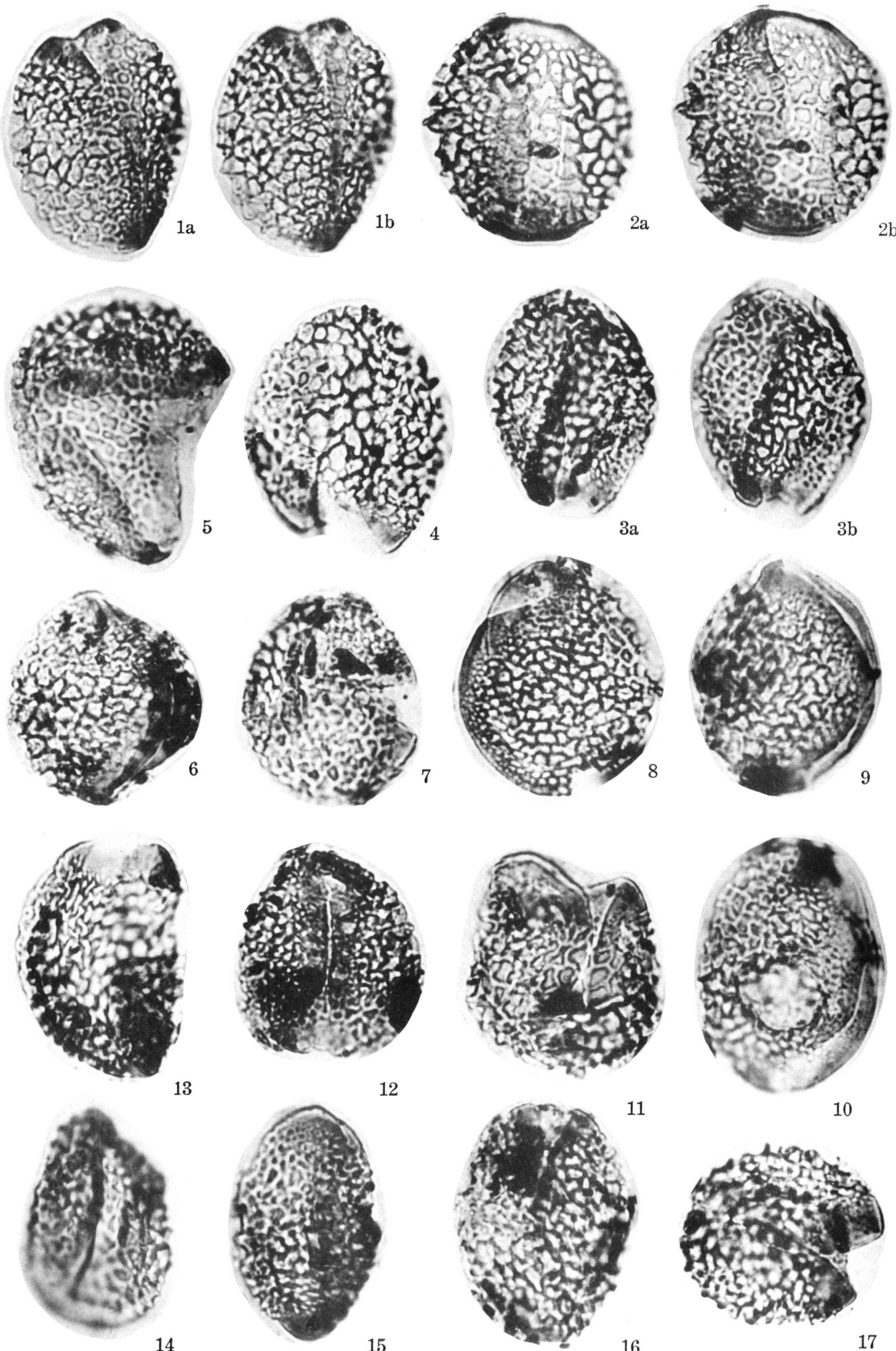

10

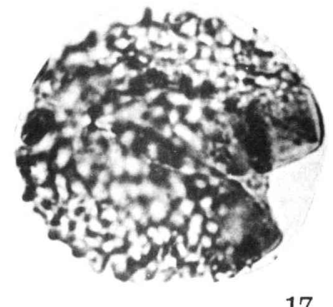

K. Takahashi : Sporen und Pollen der Hakobuchi-Schichtengruppe. 
Tafel 33 


\section{Erklärung zu Tafel 33}

(Untere Hakobuchi-Schichtengruppe von Hatsune-Sawa; ca. 833 fach vergr.)

Fig. 1-5. Monocolpopollenites shiyuparoensis $\mathrm{n}$. sp.

Fig. 1, 3, 4: Präparat GK-V 3142; Fig. 2: Präparat GK-V 3143, Holotypus; Fig. 5: Präparat GK-V 3153.

Fig. 6-8. Monocolpopollenites kyushuensis TAKaHASHI

Fig. 6: Präparat GK-V 3171; Fig. 7: Präparat GK-V 3152; Fig. 8:

Präparat GK-V 3131.

Fig. 9. Monocolpopollenites sp. a

Präparat GK-V 3131.

Fig. 10. Monocolpopollenites asymmetricus n. sp.

Präparat GK-V 3171, Holotypus.

Fig. 11. Monocolpopollenites sp. b

Präparat GK-V 3131.

Fig. 12-15. Monocolpopollenites pflugii TAKAHASHI

Fig. 12: Präparat GK-V 3141; Fig. 13: Präparat GK-V 3161; Fig. 14: Präparat GK-V 3153; Fig. 15: Präparat GK-V 3181.

Fig. 16-24. Inaperturopollenites pseudodubius TAKAHASHI

Fig. 16-19: Präparat GK-V 3131; Fig. 20: Präparat GK-V 3133; Fig. 21: Präparat GK-V 3141; Fig. 22, 23: Präparat GK-V 3142; Fig. 24: Präparat GK-V 3181.

Fig. 25. Graminidites microapertus n. sp.

Präparat GK-V 3131, Holotypus. 

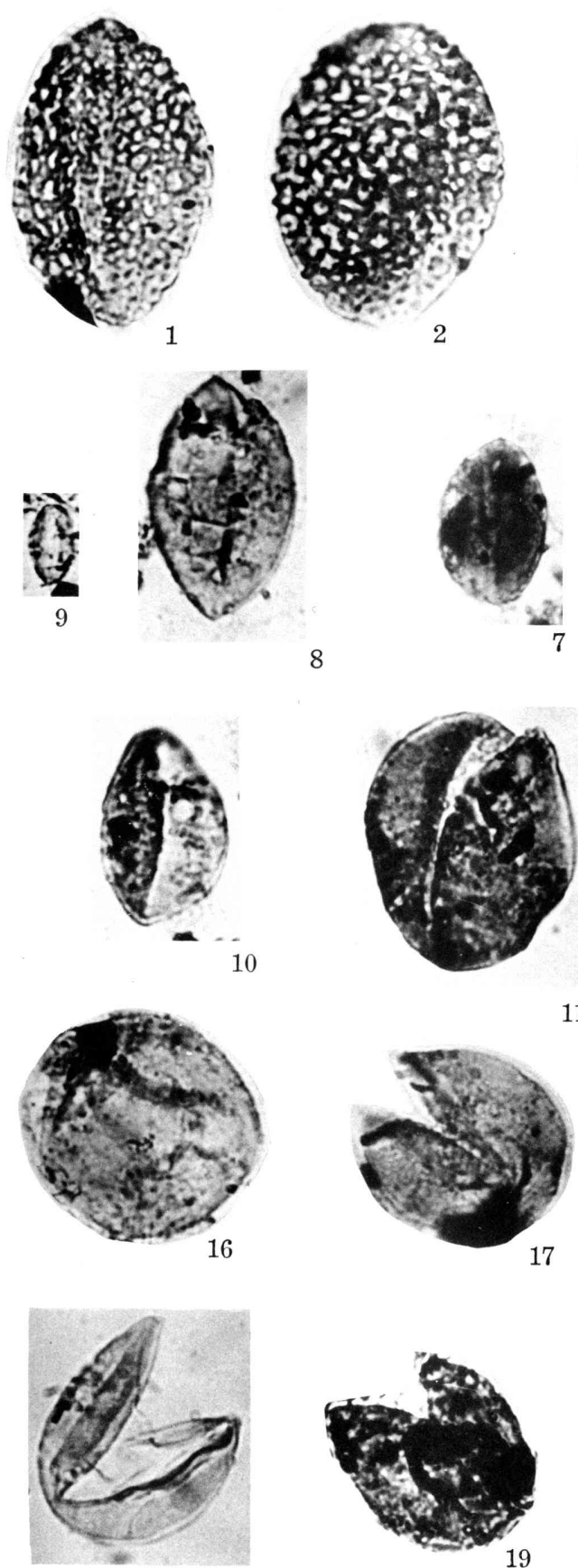

11
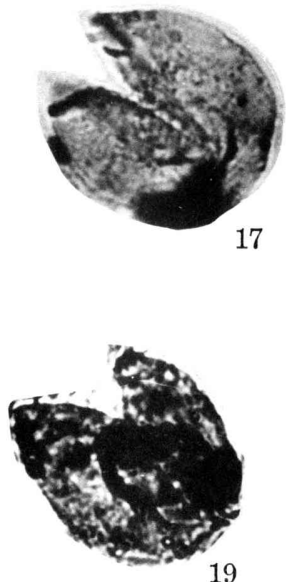

18
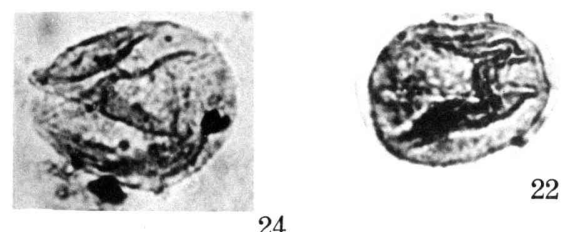

24
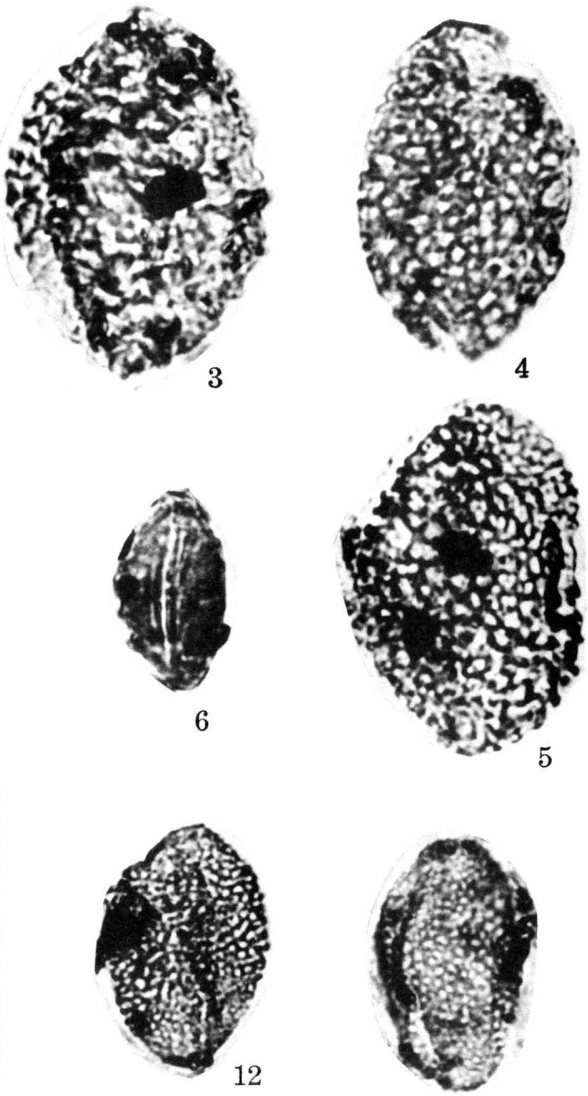

13
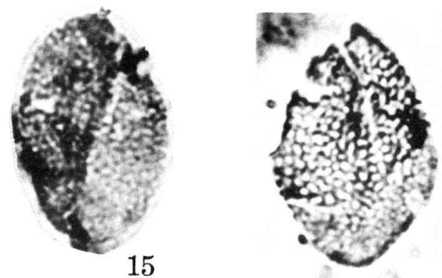

14
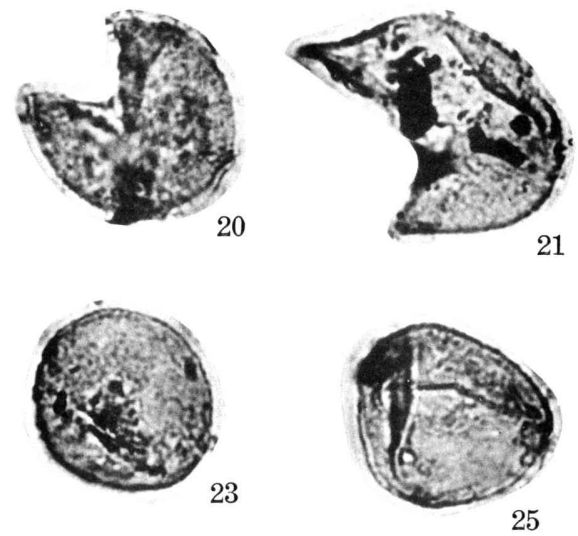

K. Takahashi : Sporen und Pollen der Hakobuchi-Schichtengruppe. 
Tafel 34 


\section{Erklärung zu Tafel 34}

(Untere Hakobuchi-Schichtengruppe von Hatsune-Sawa; ca. 833 fach vergr.)

Fig. 1, 2, 4. Pityosporites aliformis $\mathrm{n}$. sp.

Fig. 1: Präparat GK-V 3132; Fig. 2: Präparat GK-V 3131; Fig. 4:

Präparat GK-V 3143, Holotypus.

Fig. 3. Piceaepollenites saccellus $\mathrm{n}$. sp.

Präparat GK-V 3132.

Fig. 5a, b. Pityosporites sp.

Präparat GK-V 3131. 

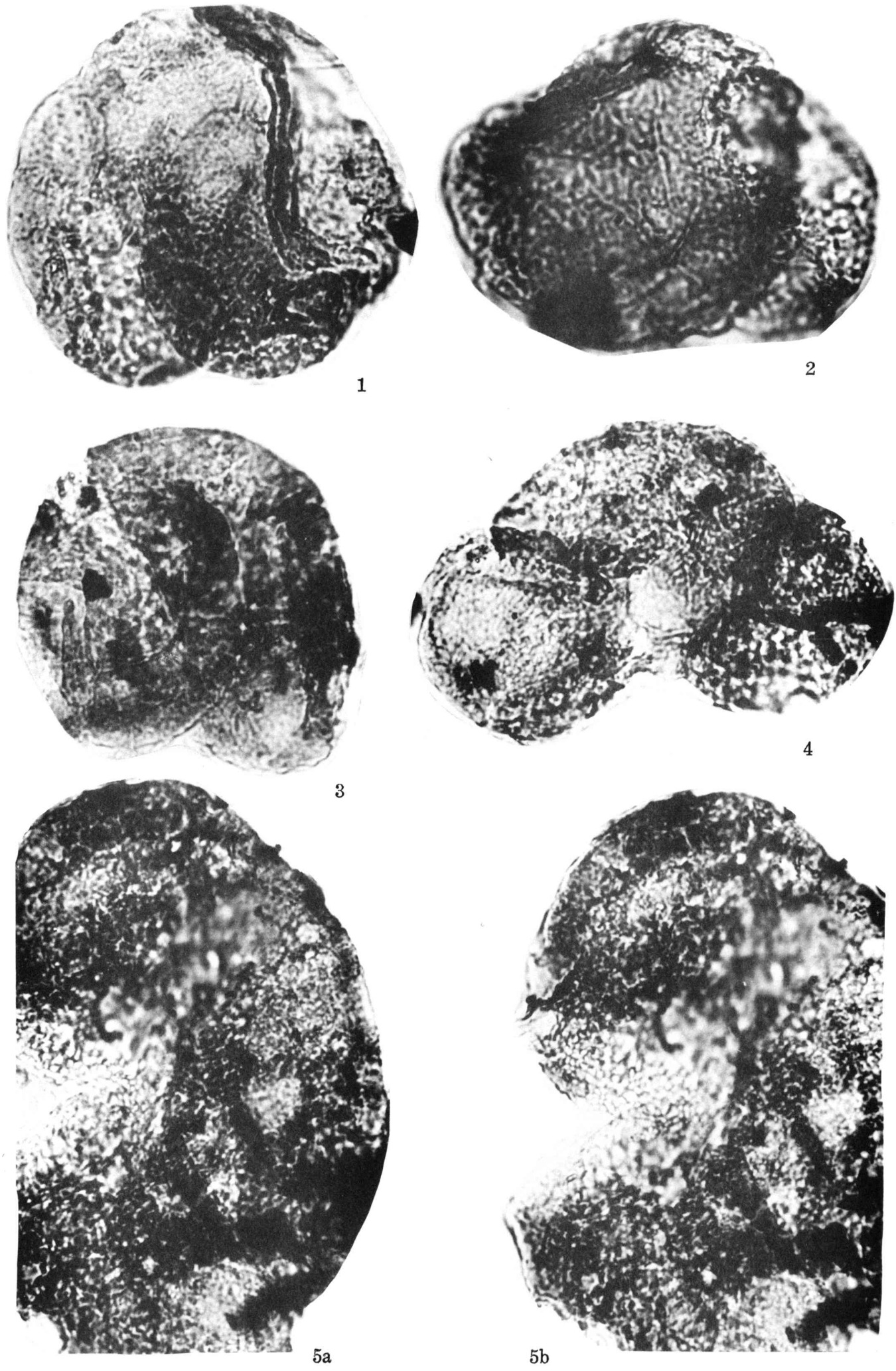

K. Takahashi: Sporen und Pollen der Hakobuchi-Schichtengruppe. 
Tafel 35 


\section{Erklärung zu Tafel 35}

(Untere Hakobuchi-Schichtengruppe von Hatsune-Sawa; ca. 833 fach vergr.)

Fig. 1, 2, 7. Piceaepollenites saccellus n. sp.

Fig. 1, 2: Präparat GK-V 3142; Fig. 1: Holotypus; Fig. 7: Präparat GK-V 3132.

Fig. 3, 5, 6. Pityosporites microaliformis n. sp.

Fig. 3: Präparat GK-V 3132, Holotypus; Fig. 5: Präparat GK-V 3161;

Fig. 4. Podocarpidites sp. a

Präparat GK-V 3131.

Fig. 8. Podocarpidites sp. b

Präparat GK-V 3152.

Fig. 9, 10, 14. Phyllocladidistes brachypterus n. sp.

Fig. 9: Präparat GK-V 3132, Holotypus; Fig. 10: Präparat GK-V 3141; Fig. 14?: Präparat GK-V 3133.

Fig. 11-13. Phyllocladidites mirandus n. sp.

Fig. 11: Präparat GK-V 3155; Fig. 12: Präparat GK-V 3161; Fig. 13: Präparat GK-V 3152, Holotypus. 

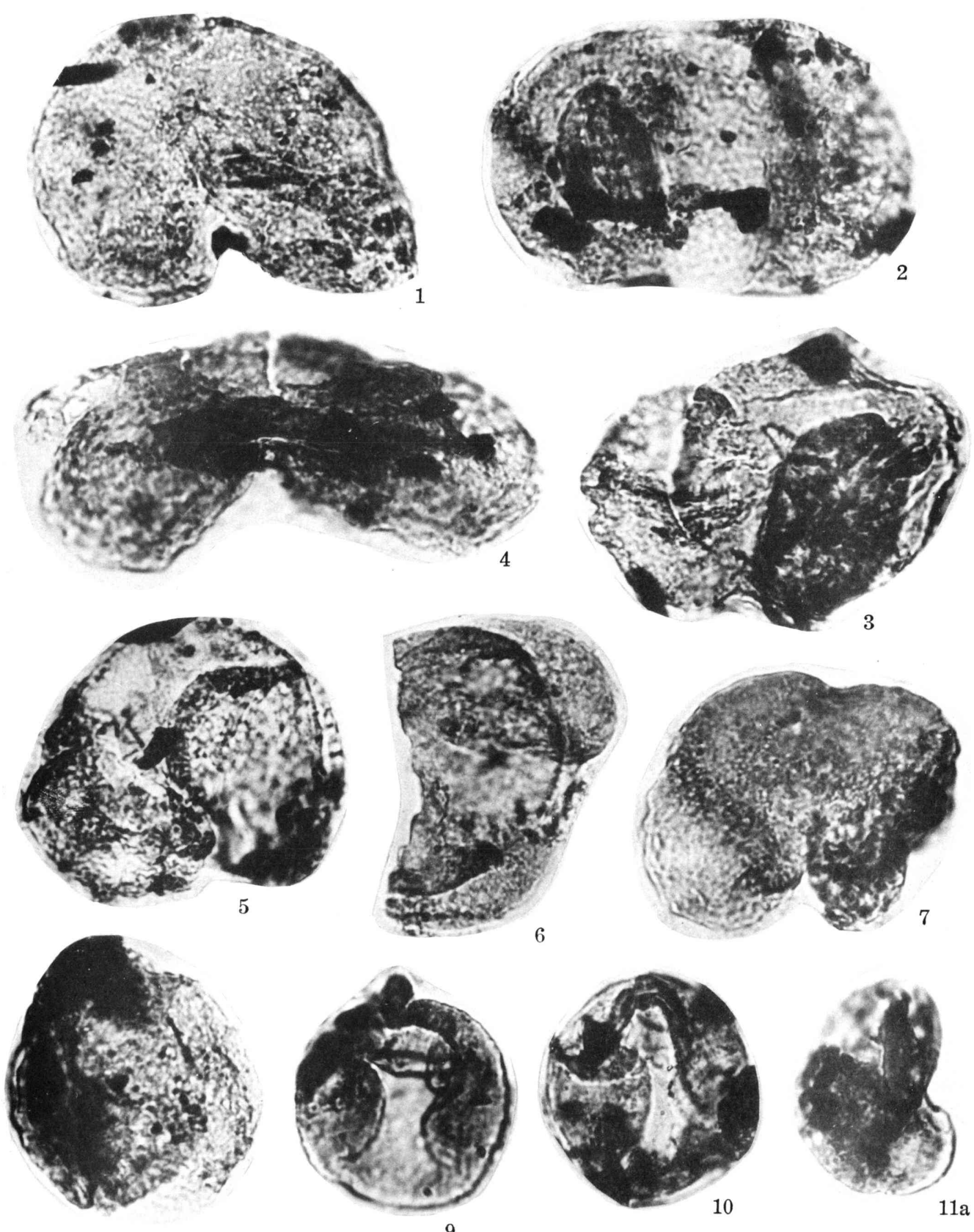

8
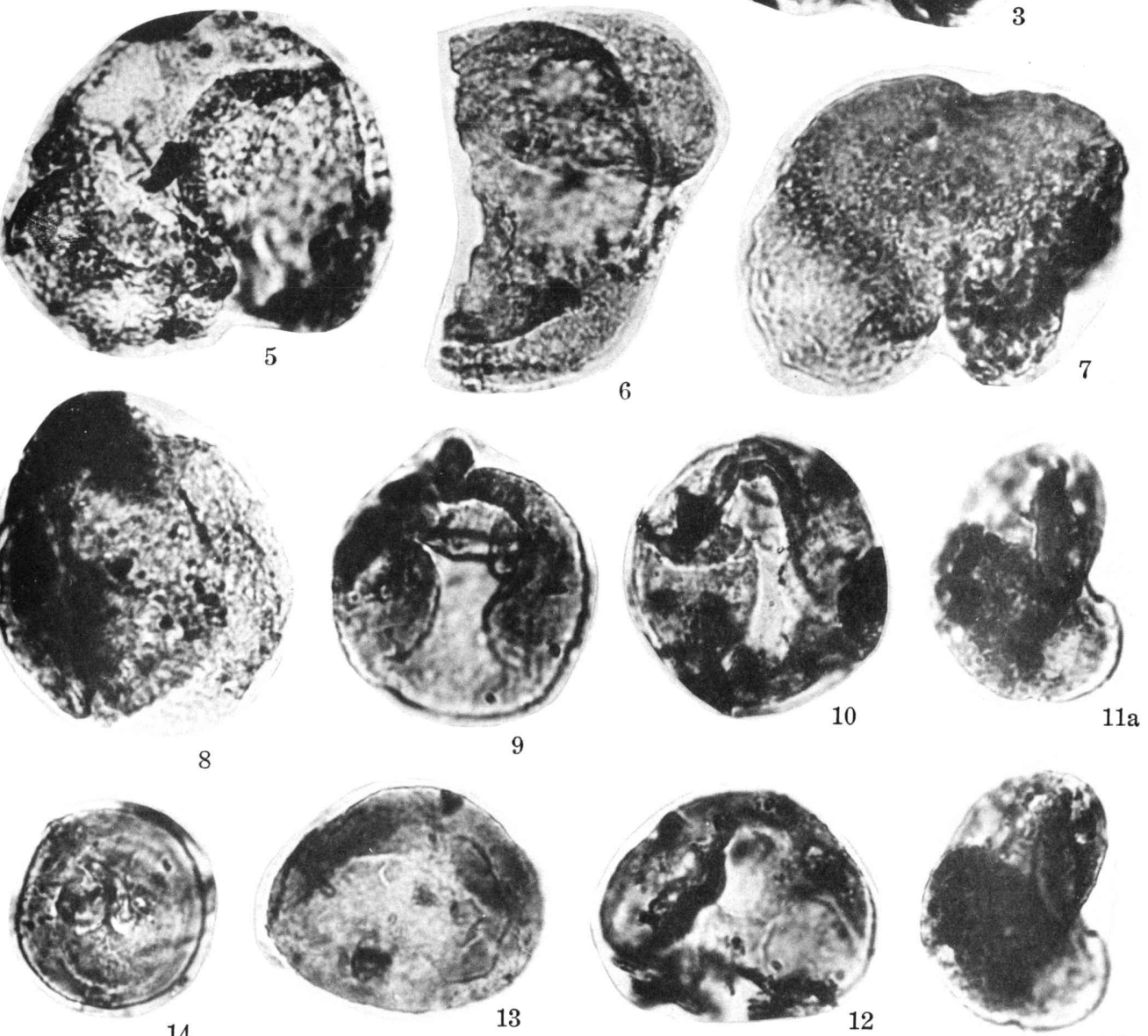

K. Takahashi : Sporen und Pollen der Hakobuchi-Schichtengruppe. 
Tafel 36 


\section{Erklärung zu Tasel 36}

(Untere Hakobuchi-Schichtengruppe von Hatsune-Sawa; ca. 833 fach vergr.)

Fig. 1-14. Alnipollenites eminens (TAKAHASHI) n. comb.

Fig. 1: Präparat GK-V 3152; Fig. 2, 3, 11, 14: Präparat GK-V 3131;

Fig. 4-7, 12, 13: Präparat GK-V 3141; Fig. 8, 9: Präparat GK-V 3142;

Fig. 10: Präparat GK-V 3143.

Fig. 15,16. Momipites constatus (TAKAHASHI) n. comb.

Fig. 15: Präparat GK-V 3181; Fig. 16: Präparat GK-V 3142.

Fig. 17. Triporopollenites festatus TAKAHASHI

Präparat GK-V 3154.

Fig. 18. Triporopollenites kasuyaensis TAKAHASHI

Präparat GK-V 3181.

Fig. 19. Subtriporopollenites kyushuensis TAKAHASHI Präparat GK-V 3153.

Fig. 20-27. Betulaepollenites normalis n. sp.

Fig. 20, 21: Präparat GK-V 3161; Fig. 22, 27?: Präparat GK-V 3131; Fig. 22a, b: Holotypus; Fig. 23: Präparat GK-V 3172; Fig. 24: Präparat GK-V 3133; Fig. 25: Präparat GK-V 3181; Fig. 26: Präparat GJi$\mathrm{V} 3155$.

Fig. 28-31. Pentapollenites yezoensis n. sp.

Fig. 28, 29: Präparat GK-V 3132; Fig. 29: Holotypus; Fig. 30a, b: Präparat GK-V 3131; Fig. 31: Präparat GK-V 3141. 

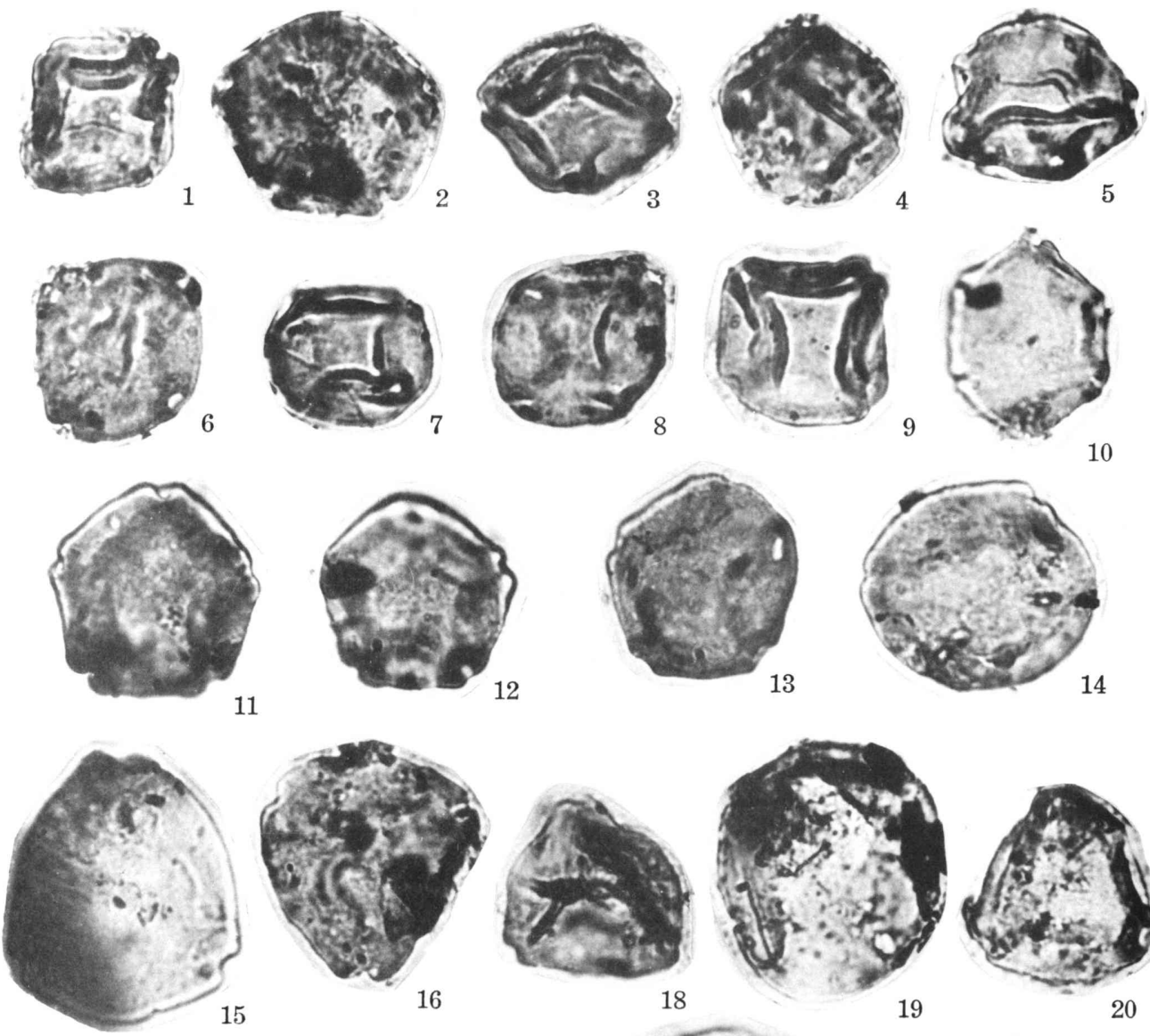

16
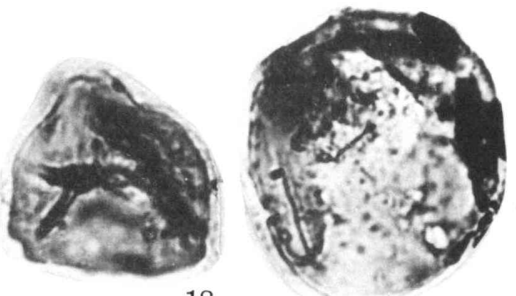

18

19
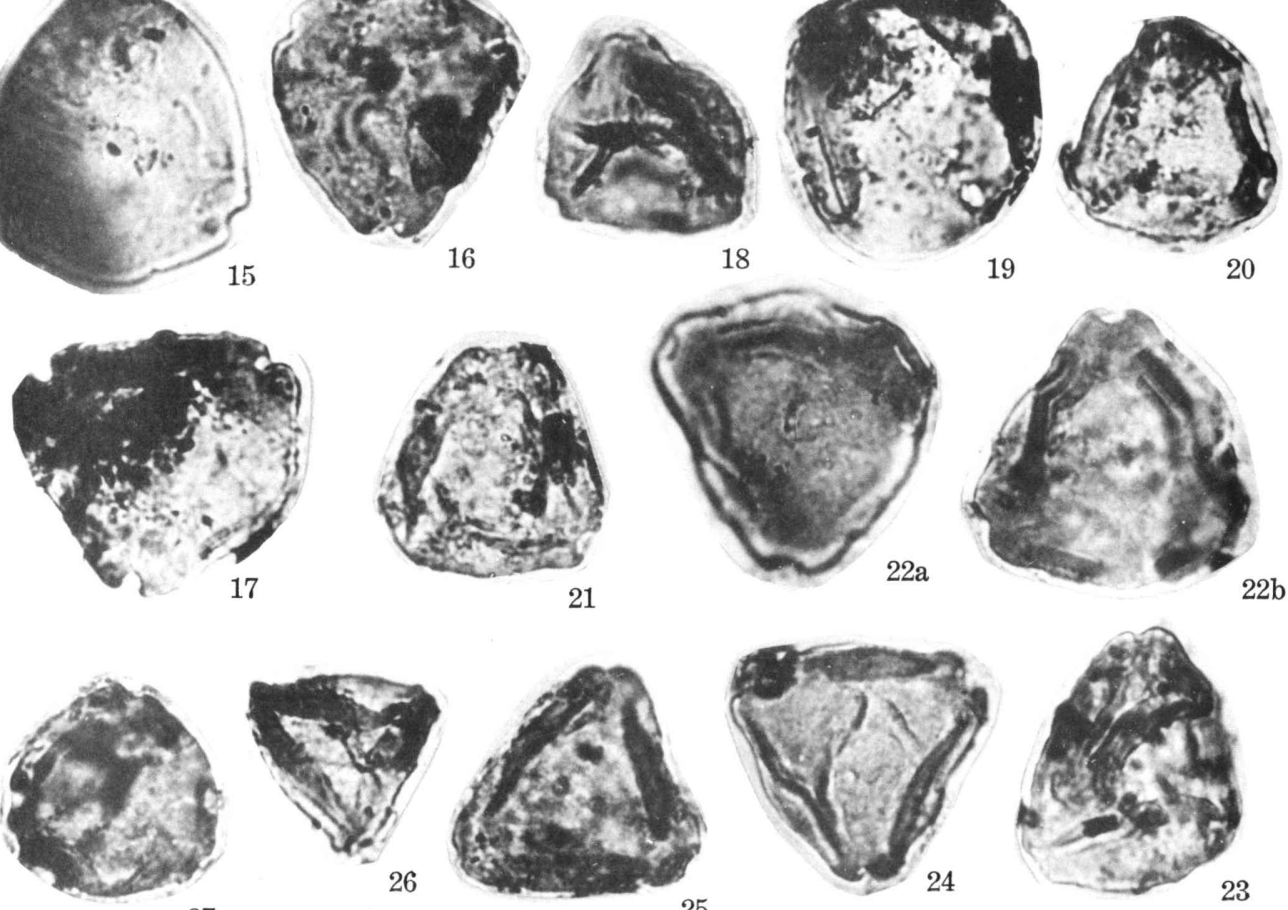

27

25
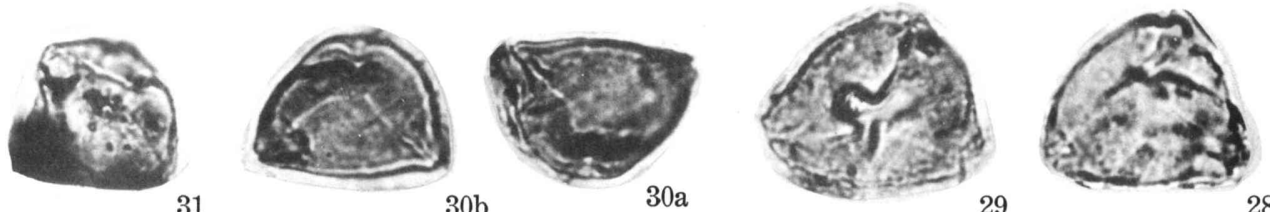

K. Takahashi: Sopren und Pollen der Hakobuchi-Schichtengruppe. 
Tafel 37 


\section{Erklärung zu Tafel 37}

(Untere Hakobuchi-Schichtengruppe von Hatsune-Sawa; ca. 833 fach vergr.)

Fig. 1a-c, 2a-c. Aquilapollenites triauritus n. sp.

Fig. 1a-c: Präparat GK-V 3131; Fig. 2a-c: Präparat GK-V 3132, Holotypus.

Fig. 3a-d, 4a-b. Aquilapollenites borealis n. sp.

Präparat GK-V 3131; Fig. 3a-d: Holotypus.

Fig. 5a, b. Aquilapollenites sp. a

Präparat GK-V 3131.

Fig. 6. ? Aquilapollenites sp. Präparat GK-V 3142.

Fig. 7a-c. Aquilapollenites sp. b Präparat GK-V 3152.

Fig. 8a-c. Aquilapollenites sp. c Präparat GK-V 3153. 

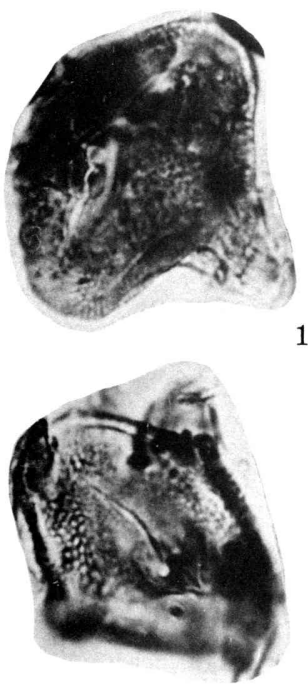

$2 \mathrm{a}$
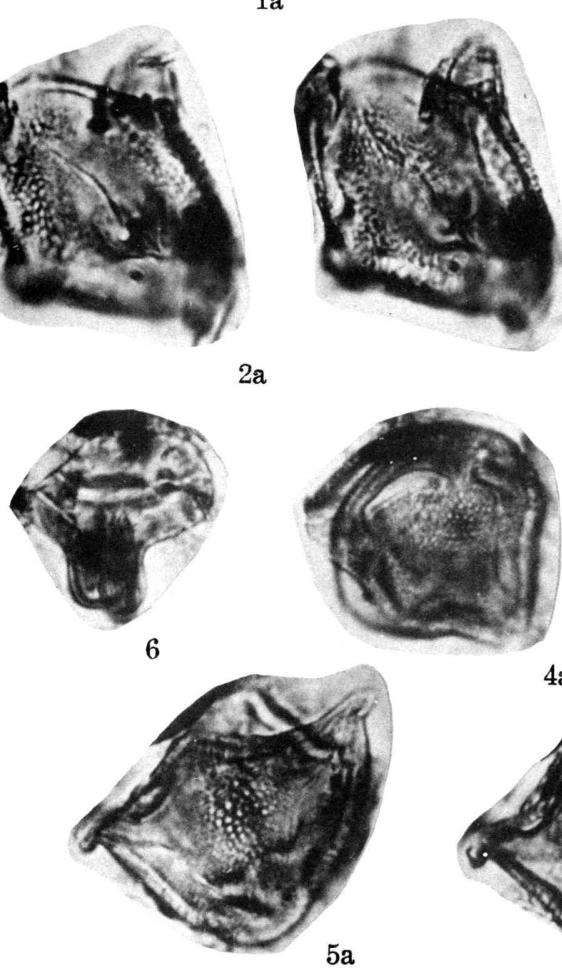

$1 b$
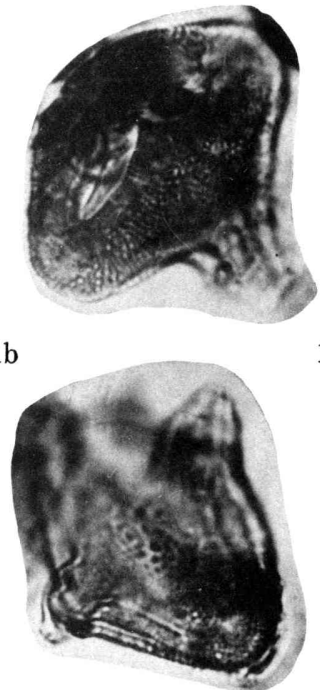

$2 b$

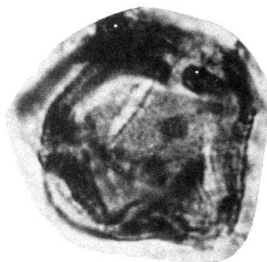

(1)

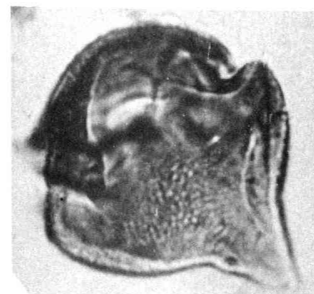

$3 a$

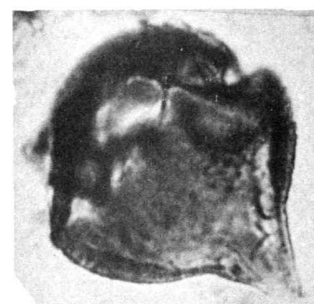

$3 b$

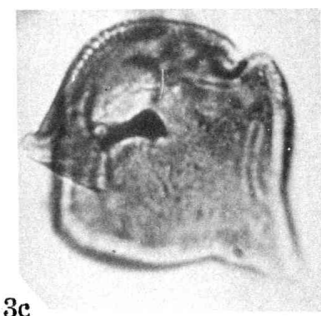

$3 c$

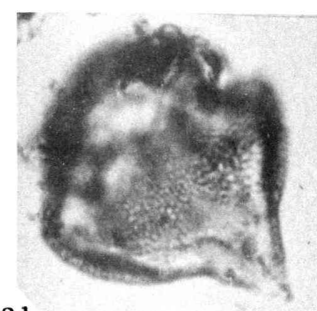

3d

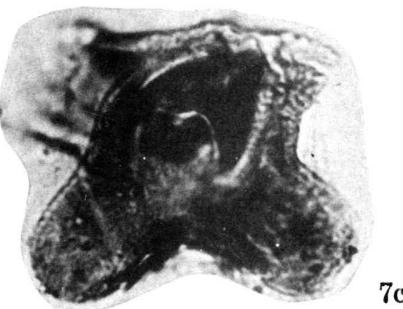

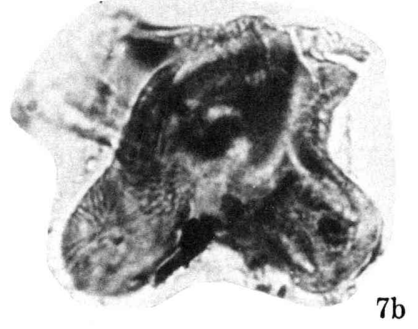
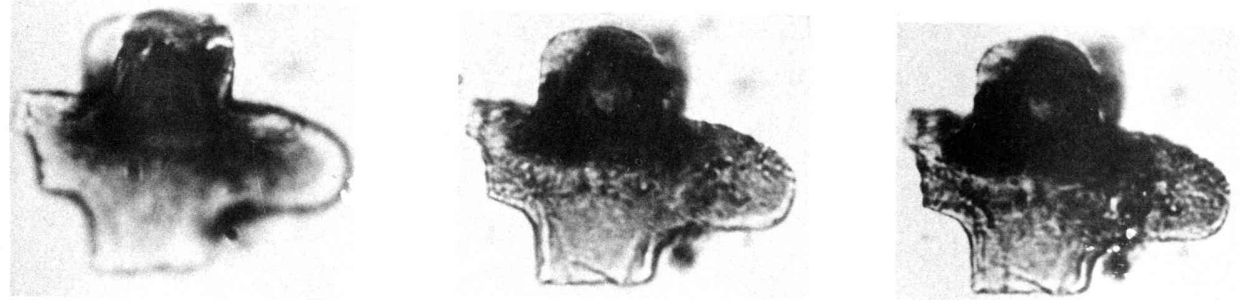

$8 \mathrm{~b}$

$8 c$

K. Takahashi: Sporen und Pollen der Hakobuchi-Schichtengruppe. 
Tafel 38 


\section{Erklärung zu Tafel 38}

(Untere Hakobuchi-Schichtengruppe von Hatsune-Sawa; ca. 833 fach vergr.)

Fig. 1a-b, 3a-b. Aquilapollenites matsumotoi n. sp.

Fig. 1a, b: Präparat GK-V 3181, Holotypus; Fig. 3a, b: Präparat GK-V 3132 .

Fig. 2a, b. Aquilapollenites sp. d

Präparat GK-V 3181.

Fig. 4-27. Tricolpopollenites minutiretiformis n. sp.

Fig. 4-8, 21, 22, 24, 25: Präparat GK-V 3131; Fig. 9-13: Präparat GKV 3141; Fig. 14-17: Präparat GK-V 3142; Fig. 17: Holotypus; Fig. 18: Präparat GK-V 3143; Fig. 19: Präparat GK-V 3152; Fig. 20: Präparat GK-V 3153; Fig. 23: Präparat GK-V 3172; Fig. 26: Präparat GK-V 3161; Fig. 27: Präparat GK-V 3132. 

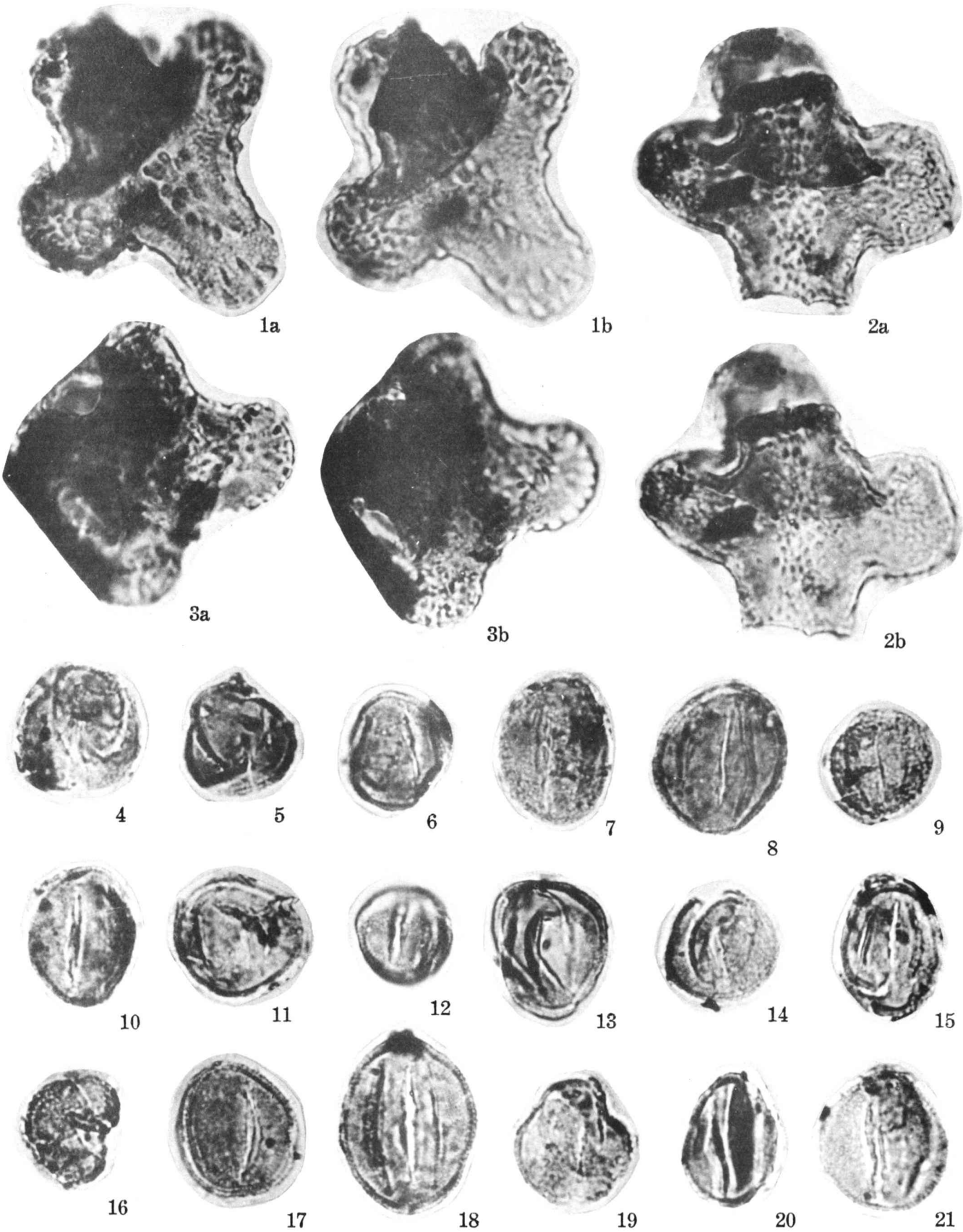

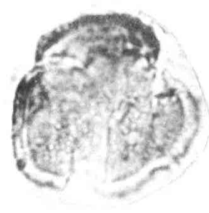

22

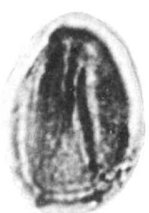

23

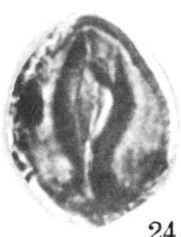

24

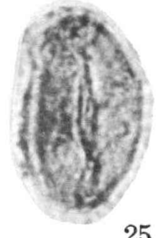

25

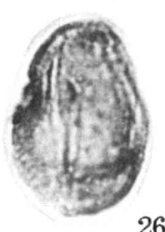

26

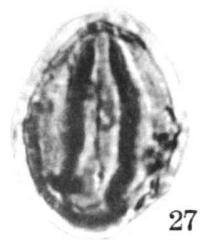

K. Takahashi: Sporen und Pollen der Hakobuchi-Schichtengruppe. 
Tafel 39 


\section{Erklärung zu Tafel 39}

(Untere Hakobuchi-Schichtengruppe von Hatsune-Sawa; ca. 833 fach vergr.)

Fig. 1. Tricolpopollenites umiensis TAKAHASHI

Präparat GK-V 3131.

Fig. 2. Tricolpopollenites cf. ditis TAKAHASHI

Präparat GK-V 3161.

Fig. 3, 6. Tricolpopollenites meinohamensis TAKAHASHI subsp. rotundus TAKAHASHI Präparat GK-V 3131; Fig. 6 ?

Fig. 4. Tricolpopollenites ef. microretsculatus TAKAHASH Präparat GK-V 3141.

Fig. 5a, b. Tricolpopollenites sp. a Präparat GK-V 3131.

Fig. 7. Tricolpopollenites cf. subasper TAKAHASHI Präparat GK-V 3153.

Fig. 8. Tricolpopollenites sp. b Präparat GK-V 3131.

Fig. 9. Tricolpopollenites sp. c Präparat GK-V 3152.

Fig. 10, 11. Tricolpopollenites conicus n. sp. Fig. 10: Präparat GK-V 3131, Holotypus; Fig. 11: Präparat GK-V 3153.

Fig. 12. Tricolpopollenites sp. d Präparat GK-V 3161.

Fig. 13. Tricolpopollenites sp. $\mathrm{g}$ Präparat GK-V 3142.

Fig. 14. Tricolpopollenites sp. h Präparat GK-V 3161.

Fig. 15. Tricolpopollenites sp. i Präparat GK-V 3131.

Fig. 16-18. Tricolporopollenites minor TAKAHASHI

Fig. 16: Präparat GK-V 3142; Fig. 17: Präparat GK-V 3161; Fig. 18: Präparat GK-V 3171.

Fig. 19. Tricolporopollenites sp. a Präparat GK-V 3161.

Fig. 20. Tricolporopollenites sp. b Präparat GK-V 3131.

Fig. 21-26. Rhoipites ? minutireticulatus n. sp.

Fig. 21, 23: Präparat GK-V 3131; Fig. 22, 24: Präparat GK-V 3141;

Fig. 25: Präparat GK-V 3161; Fig. 26: Präparat GK-V 3143, Holotypus.

Fig. 27. Tricolporopollenites sp. c Präparat GK-V 3131.

Fig. 28. Unbestimmbarer Pollen

Präparat GK-V 3152.

Fig. 29. Tricolporopollenites sp. d Präparat GK-V 3131.

Fig. 30-33. Tetracolporopollenites cretaceus n. sp. Fig. 30?: Präparat GK-V 3132; Fig. 31a, b: Präparat GK-V 3161; Fig. 32: Präparat GK-V 3142; Fig. 33a, b: Präparat GK-V 3152, Holotypus. 

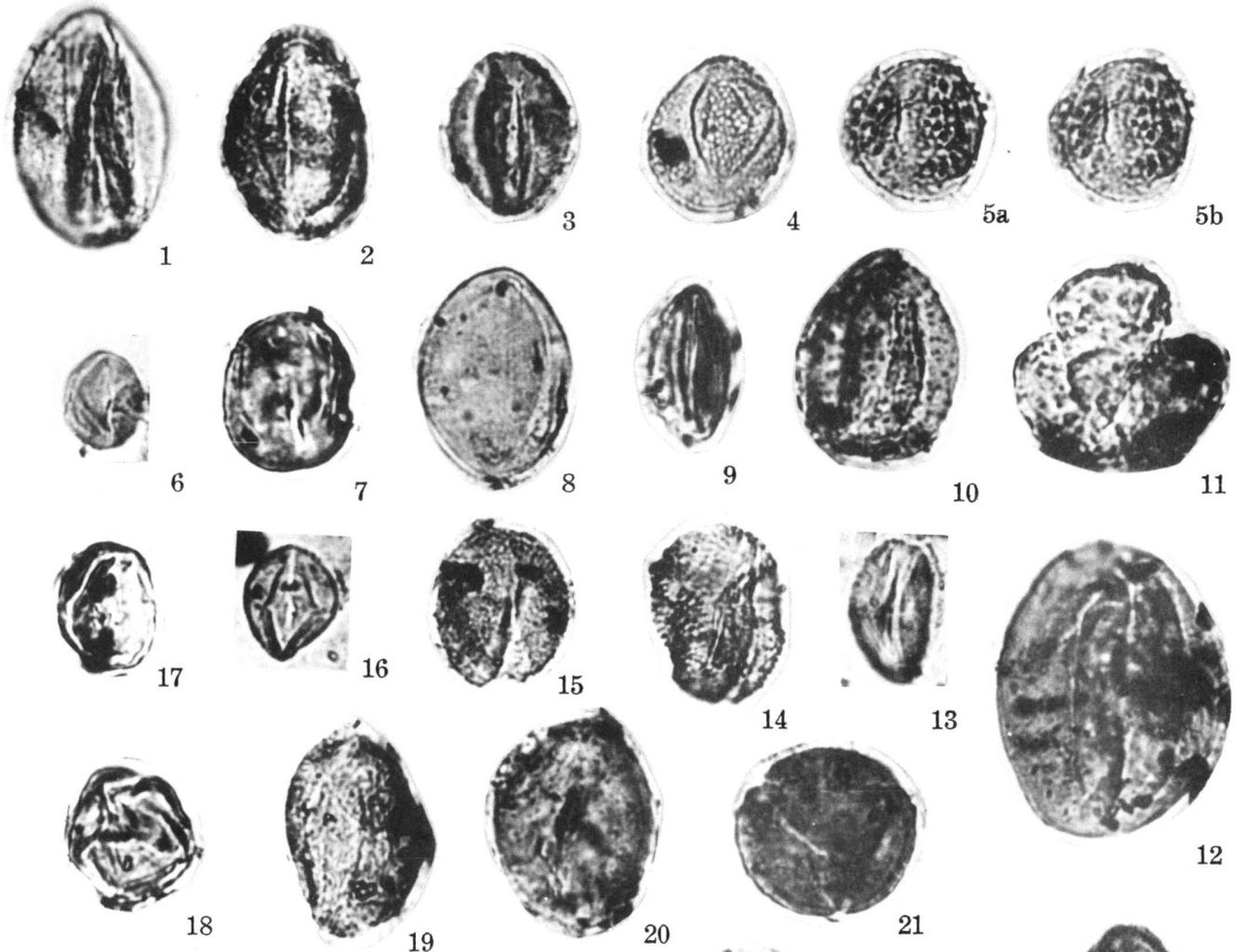

13
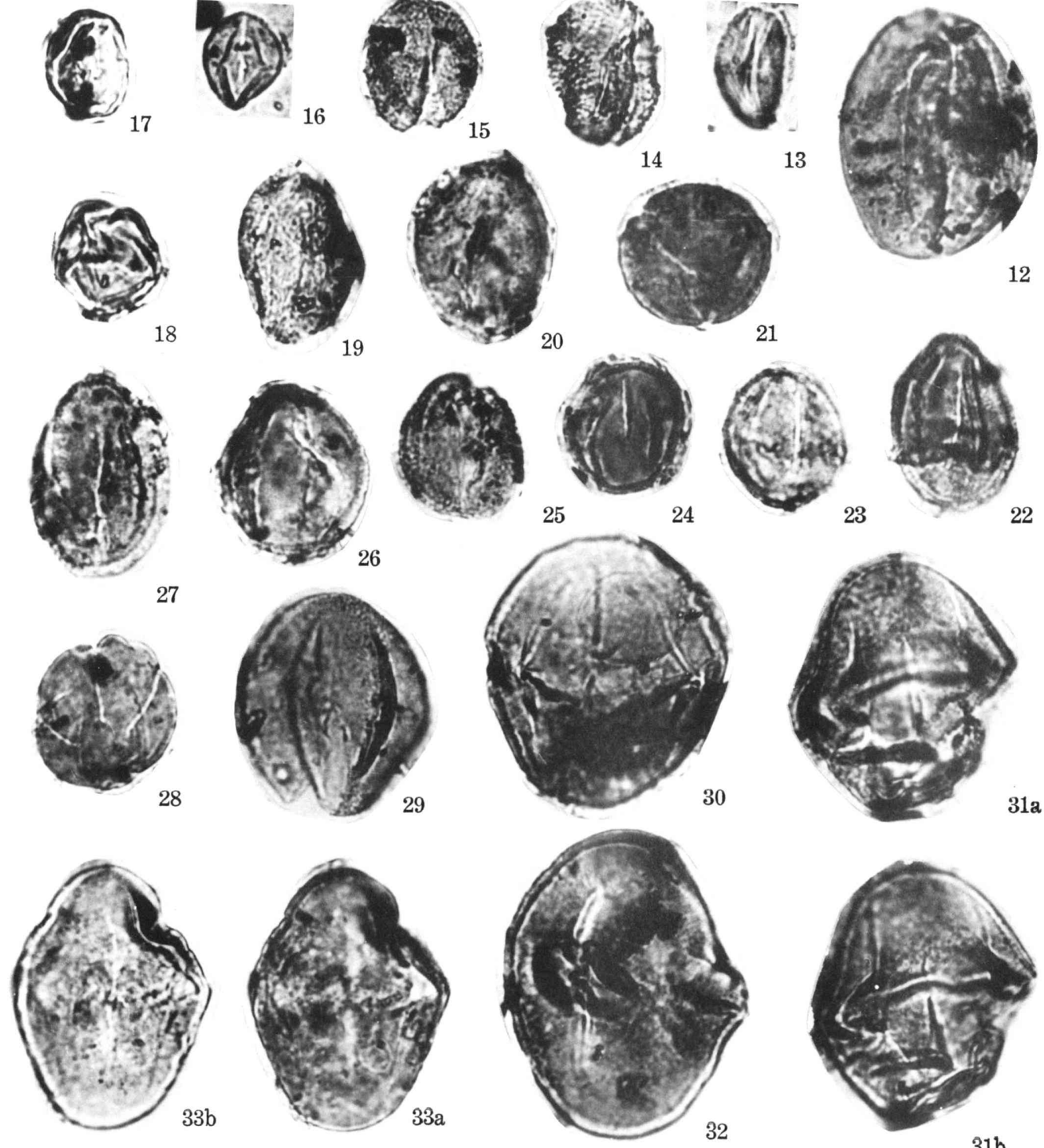

K. Takahashi : Sporen und Pollen der Hakobuchi-Schichtengruppe. 
Tafel 40 


\section{Erklärung zu Tafel 40}

(Obere Hakobuchi-Schichtengruppe bei der Talspere von Ooyubari; ca. 833 fach vergr.)

Fig. 1. Gleicheniidites marginatus n. sp.

Präparat GK-V 3213.

Fig. 2. ? Concavisporites sp.

Präparat GK-V 3245.

Fig. 3a, b. Concavisporites sp. b

Präparat GK-V 3214.

Fig. 4a, b. Biretisporites? minus n. sp.

Präparat GK-V 3219.

Fig. 5. Corrugatisporites cf. solidus (R. Рот.) Thomson \& Pflug Präparat GK-V 3211.

Fig. 6, 7a, b. Lycopodiumsporites yubariensis n. sp.

Fig. 6: Präparat GK-V 3213; Fig. 7a, b: Präparat GK-V 3212, Holotypus.

Fig. 8a, b. Neoraistrickia sp.

Präparat GK-V 3220.

Fig. 9a, b. Tuberculatisporites sp.

Präparat GK-V 3211.

Fig. 10. Verrucosisporites sp. Präparat GK-V 3248.

Fig. 11a, b. Rugulatisporites cf. salebrosus n. sp. Präparat GK-V 3248.

Fig. 12,18. Laevigatosporites prominens $\mathrm{n}$. sp.

Fig. 12: Präparat GK-V 3216; Fig. 18: Präparat GK-V 3215.

Fig. 13-15. Laevigatosporites dehiscens TAKAHASHI

Fig. 13: Präparat GK-V 3248; Fig. 14, 15: Präparat GK-V 3217.

Fig. 16,17. Laevigatosporites senonicus $\mathrm{n}$. sp.

Fig. 19. Punctatosporites sp.

Präparat GK-V 3216.

Fig. 20. Polypodiisporites invisus n. sp. Präparat GK-V 3212.

Fig. 21a, b. Verrucatosporites sp. b Präparat GK-V 3249.

Fig. 22a, b. Polypodiisporites repandus n. sp. Präparat GK-V 3214. 

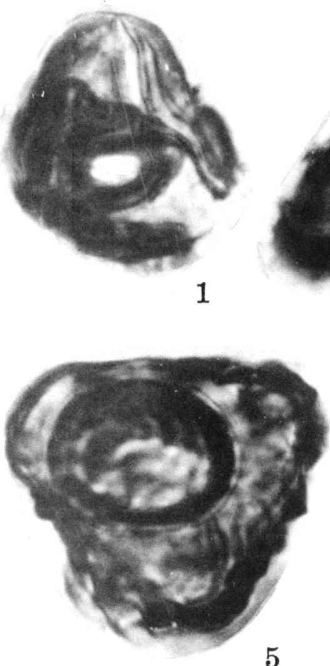

5

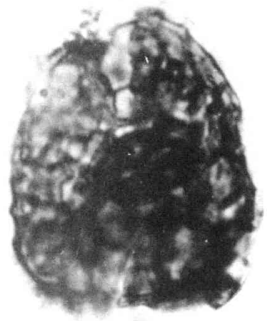

$7 \mathrm{a}$

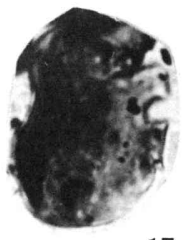

17

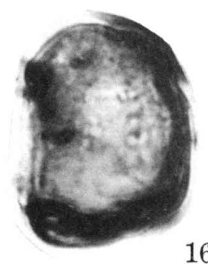

16

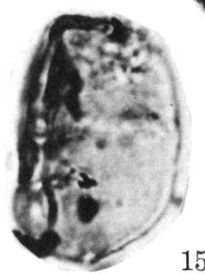

15

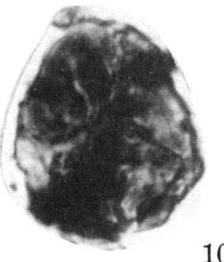

$4 \mathrm{a}$

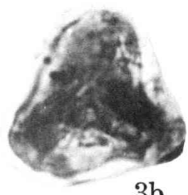

$3 b$
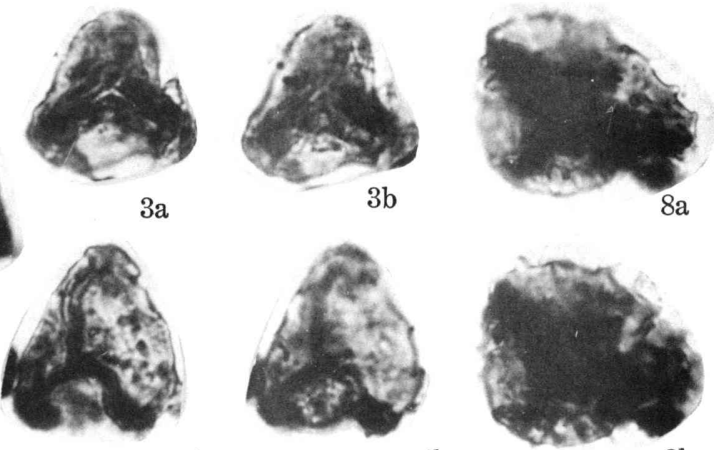

$4 \mathrm{~b}$
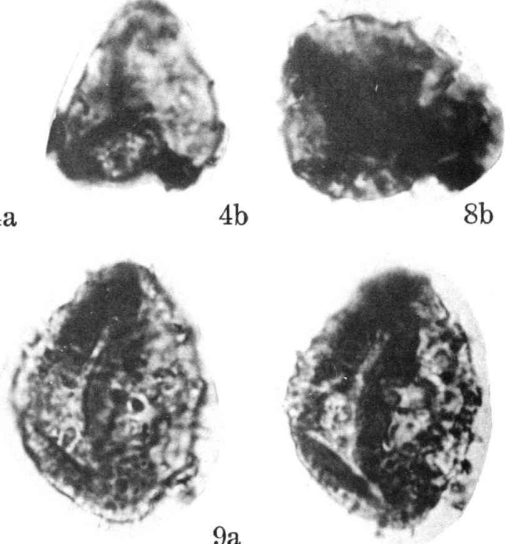

$9 \mathrm{a}$

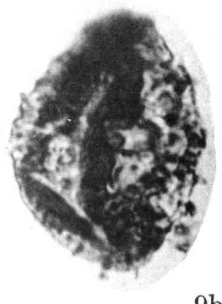

$9 \mathrm{~b}$
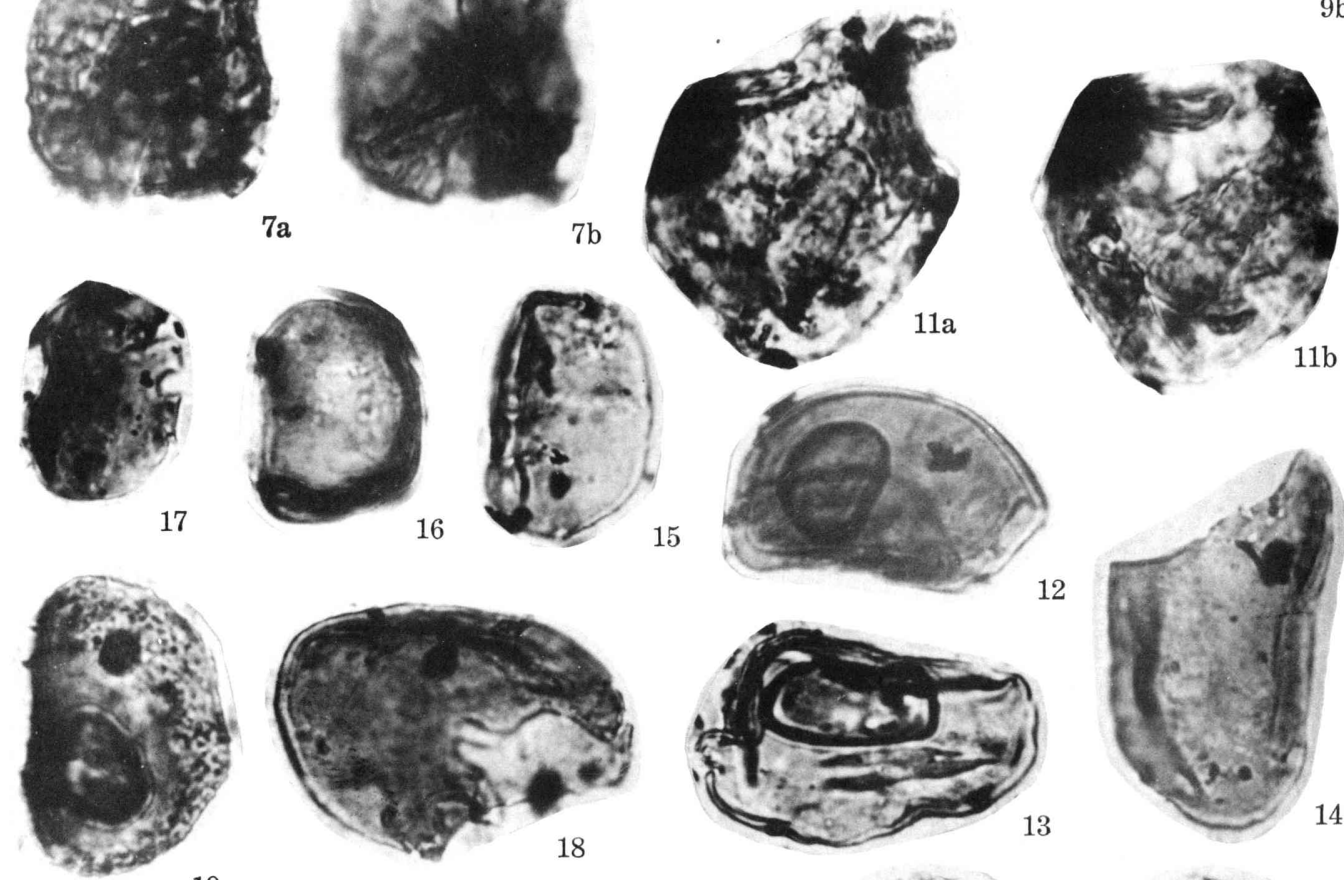

12
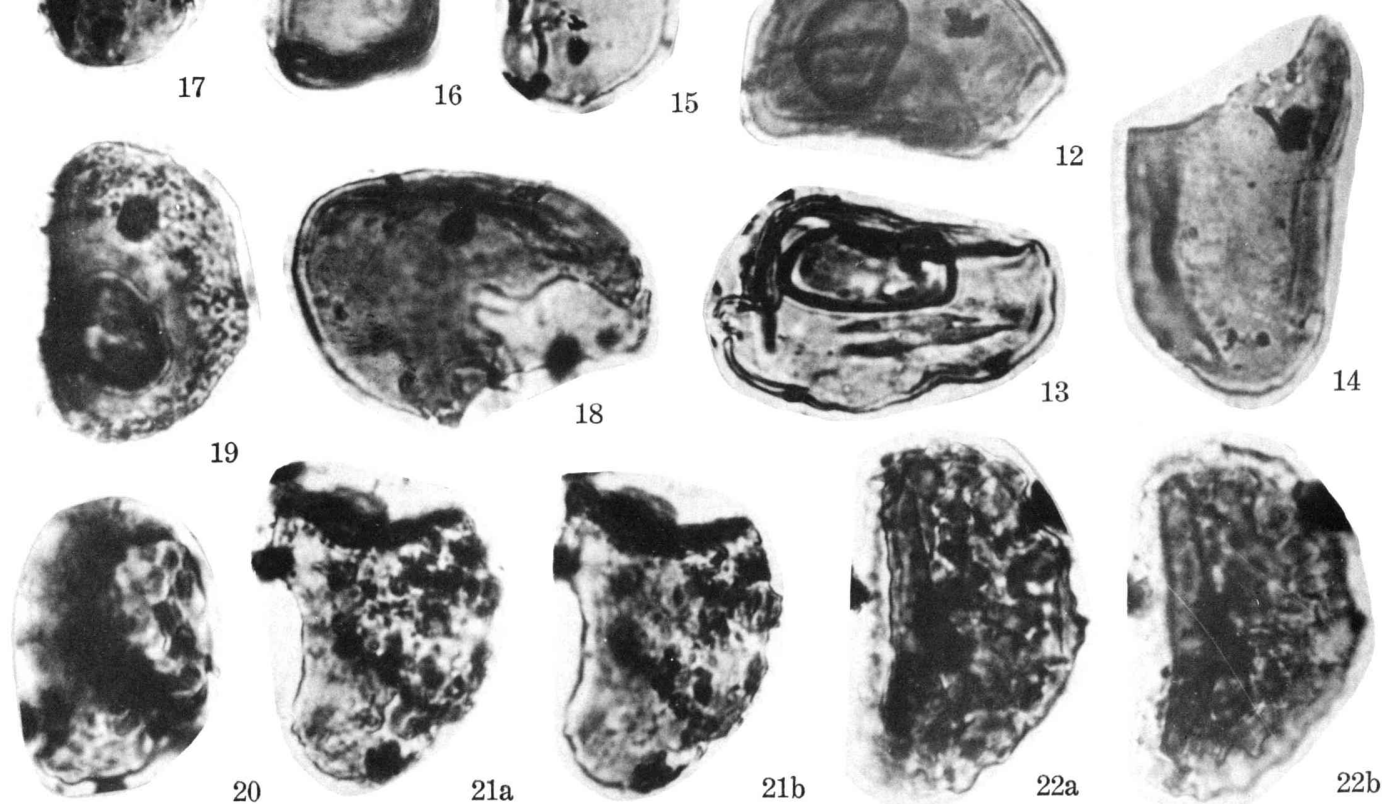

K. Takahashi : Sporen und Pollen der Hakobuchi-Schichtengruppe. 
Tafel 41 


\section{Erklärung zu Tafel 41}

(Obere Hakobuchi-Schichtengruppe bei der Talspere von Ooyubari; ca. 833 fach vergr.)

Fig. 1. Inaperturopollenites magnus (R. PotoniÉ) THomson \& PFLUG Präparat GK-V 3243.

Fig. 2, 3. Inaperturopollenites (?) falsus n. sp.

Fig. 2: Präparat GK-V 3216; Fig. 3: Präparat GK-V 3249, Holotypus.

Fig. 4. ? Tuberculatisporites sp. Präparat GK-V 3218.

Fig. 5. Inaperturopollenites pseudodubius TAKAHASHI Präparat GK-V 3212.

Fig. 6-8. Inaperturopollenites sp. (Tetradenverband) Präparat GK-V 3212.

Fig. 9-12. Inaperturopollenites parviundulatus $\mathrm{n} . \mathrm{sp}$.

Fig. 9, 10: Präparat GK-V 3245; Fig. 11a, b, 12: Präparat GK-V 3247; Fig. 11a, b: Holotypus.

Fig. 13,14: Monocolpopollenites kyushuensis TAKAHASHI

Fig. 15. Tricolpopollenites cf. ditis TAKAHASH Präparat GK-V 3214.

Fig. 16,17. Tricolpopollenites minutiretiformis $\mathrm{n}$. sp. Fig. 16: Präparat GK-V 3248; Fig. 17: Präparat GK-V 3244.

Fig. 18. Tricolpopollenites sp. e Präparat GK-V 3213.

Fig. 19. Tricolpopollenites sp. $\mathrm{f}$ Präparat GK-V 3220.

Fig. 20a, b. ? Pistillipollenites sp. Präparat GK-V 3214.

Fig. 21. Rhaipites? minutireticulatus $\mathrm{n}$. sp. Präparat GK-V 3213.

Fig. 22-25. Tricolporopollenites cf. minor TAKAHASHI

Fig. 22: Präparat GK-V 3244; Fig. 23: Präparat GK-V 3214; Fig. 24: Präparat GK-V 3212; Fig. 25?: Präparat 3211.

Fig. 26,27. Unbestimmbare Pollen Fig. 26: Präparat GK-V 3242; Fig. 27: Präparat GK-V 3216.

Fig. 28-30. Alnipollenites eminens (TAKAHASHI) n. comb. Fig. 28: Präparat GK-V 3218; Fig. 29, 30: Präparat GK-V 3212.

Fig. 31a, b. Aquilapollenites quadrinus n. sp. Präparat GK-V 3216, Holotypus. 
Mem. Fac. Sci., Kyushu Univ., Ser. D, Geology, Vol. XIV
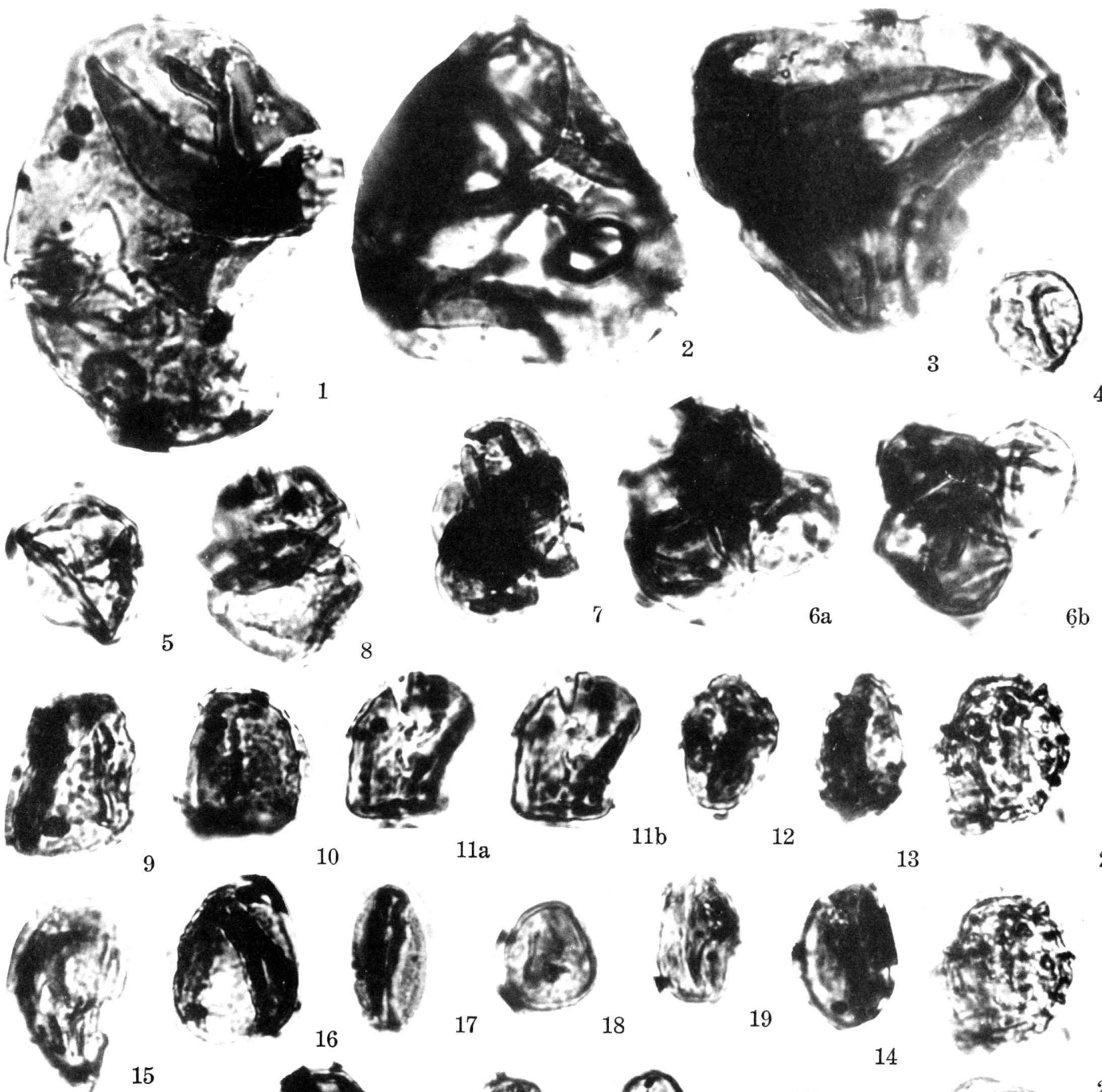

$11 \mathrm{a}$

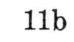

12

13

$20 \mathrm{a}$

15

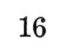

17
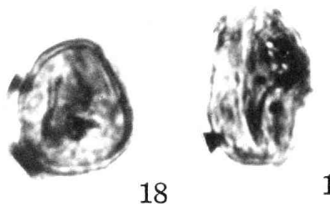

18
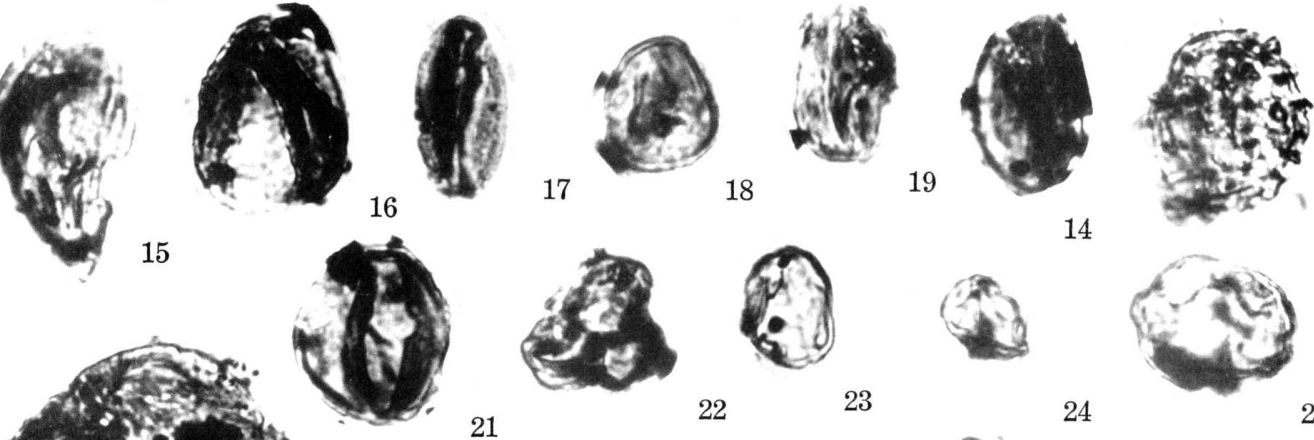

(at)

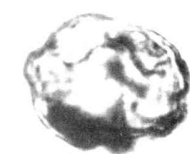

24
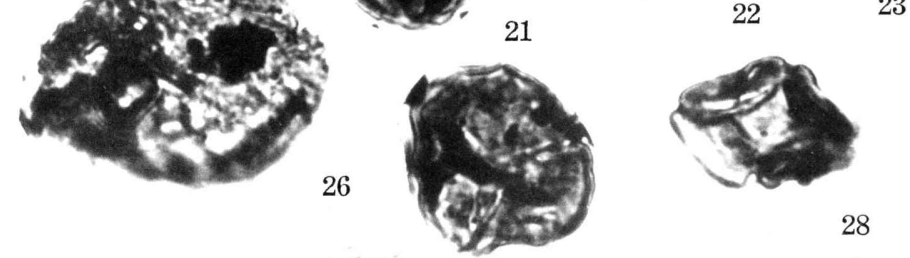

28
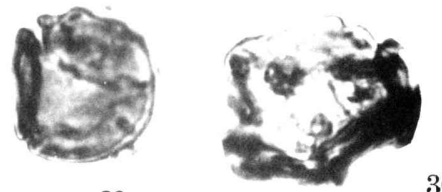

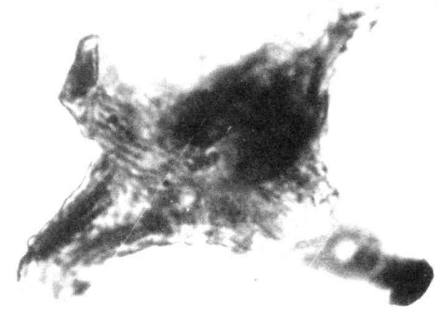

31a

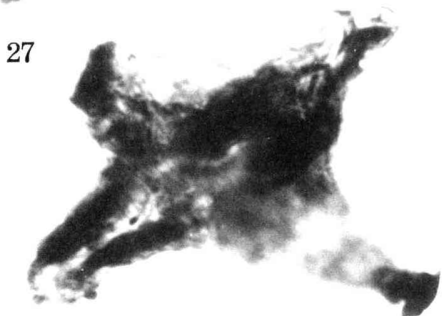

$31 b$

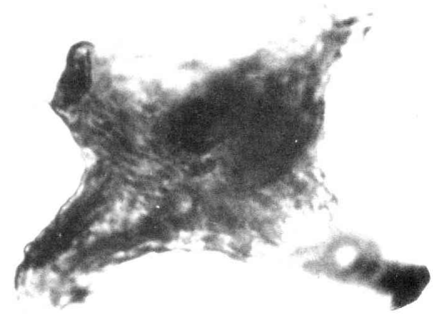

$31 \mathrm{c}$

K. Takahashi : Sporen und Pollen der Hakobuchi-Schichtengruppe. 
Tafel 42 


\section{Erklärung zu Tafel 42}

(Obere Hakobuchi-Schichtengruppe bei der Talspere von Ooyubari; ca. 833 fach vergr.)

Fig. 1-7. Betulaepollenites minutulus n. sp.

Fig. 1a, b: Präparat GK-V 3215, Holotypus; Fig. 2: Präparat GK-V 3213; Fig. 3: Präparat GK-V 3211; Fig. 4: Präparat GK-V 3243; Fig. 5a, b, 7?: Präparat GK-V 3244; Fig. 6a, b: Präparat GK-V 3251.

Fig. 8. Betulaepollenites sp. Präparat GK-V 3220.

Fig. 9a, b. Trivestibulopollenites sp. Präparat GK-V 3212.

Fig. 10. Tricolporopollenites cf. minor TAKAHASHI Präparat GK-V 3249.

Fig. 11. Momipites sp. Präparat GK-V 3213.

Fig. 12. Graminidites microapertus n. sp. Präparat GK-V 3213.

Fig. 13. Polyporopollenites sp. a Präparat GK-V 3250.

Fig. 14. Betulaepollenites normalis $\mathrm{n}$. sp. Präparat GK-V 3250.

Fig. 15. ? Trudopollis sp. Präparat GK-V 3219.

Fig. 16. ? Triporopollenites sp. Präparat GK-V 3250.

Fig. 17. Polyporopollenites $\mathrm{sp} . \mathrm{b}$ Präparat GK-V 3246.

Fig. 18. Polyporopollenites sp. c Präparat GK-V 3243.

Fig. 19, 23, 24: Polyporopollenites punctatus n. sp. Fig. 19: Präparat GK-V 3216; Fig. 23a, b: Präparat GK-V 3250; Fig.

Fig. 20. Polyporopollenites sp. $\mathrm{d}$ Präparat GK-V 3214.

Fig. 21, 22. Ulmipollenites sp.

Fig. 21: Präparat GK-V 3211; Fig. 22: Präparat GK-V 3242.

Fig. 25. Polyporopollenites sp. e Präparat GK-V 3243.

Fig. 26. Polyporopollenites sp. f Präparat GK-V 3246.

Fig. 27-30. Unbestimmbare Reste

Fig. 31-32. Unbestimmbare Reste 
Mem. Fac. Sci., Kyushu Univ., Ser. D, Geology, Vol. XIV

Tafel 42

3in

- 1 -
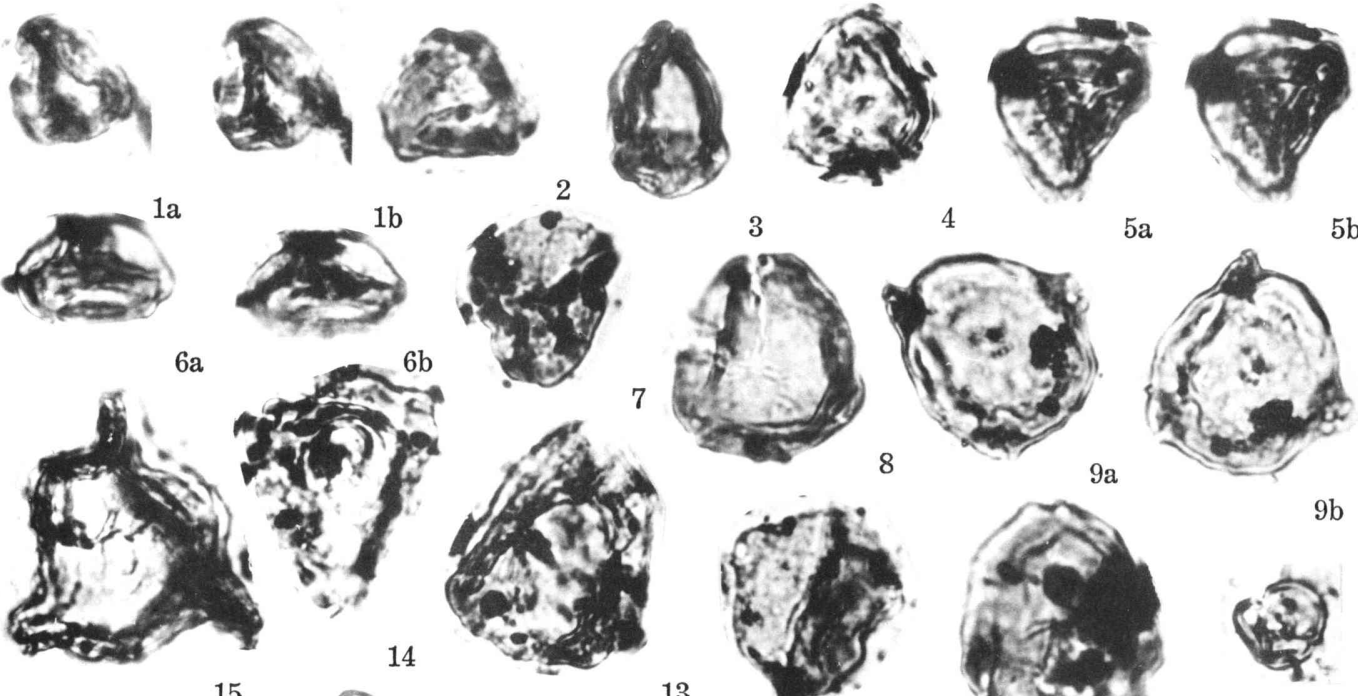

$5 \mathrm{a} \quad 5 \mathrm{~b}$
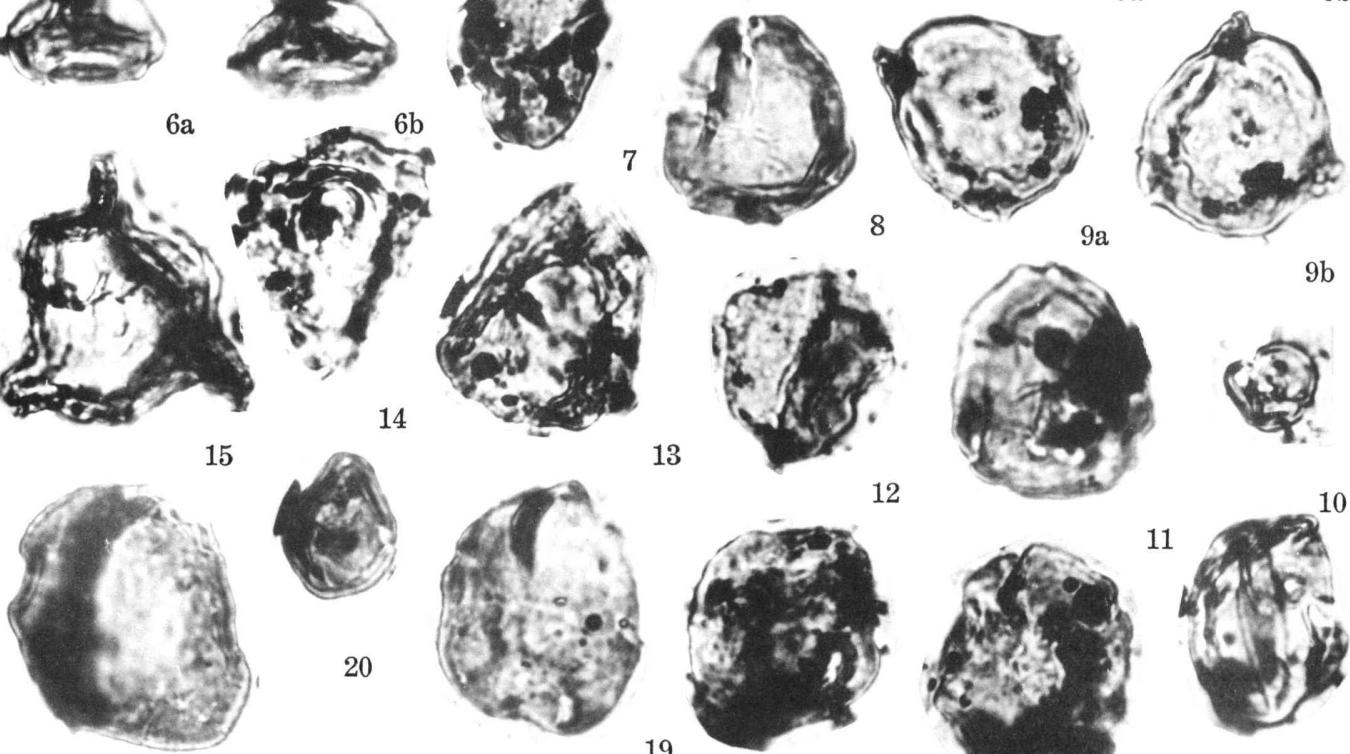

$9 \mathrm{a}$
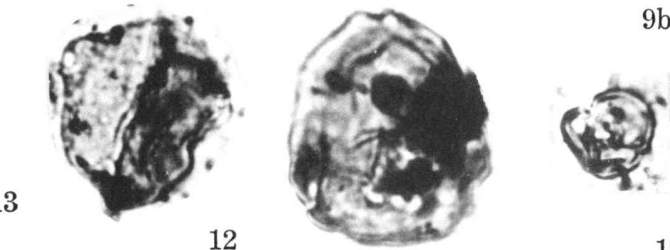

12 valiak

$9 \mathrm{~b}$
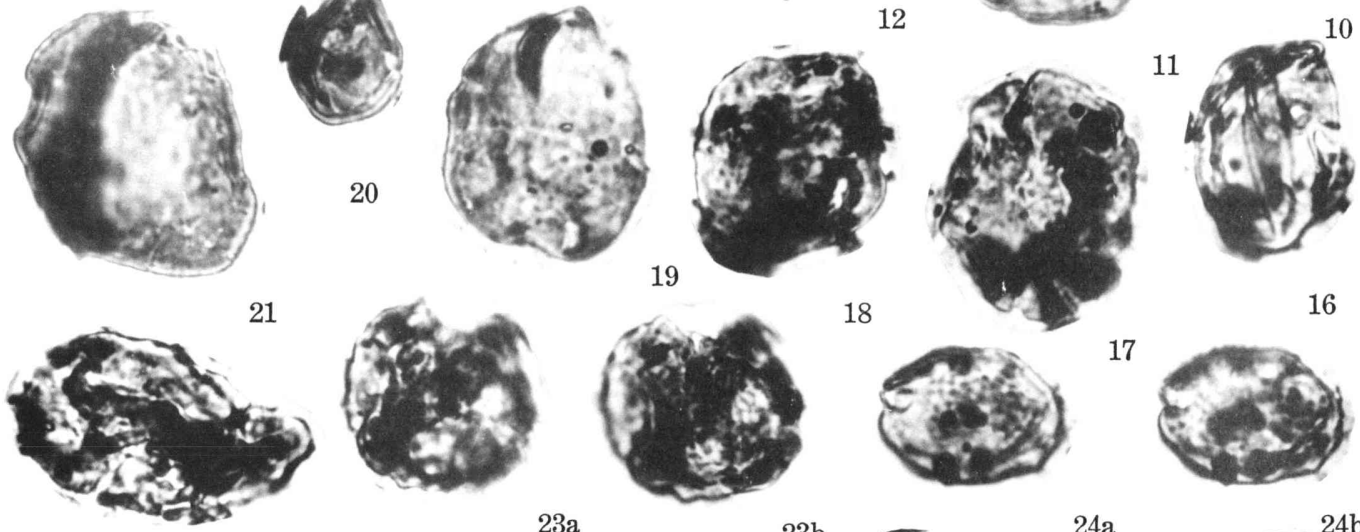

22

$23 \mathrm{a}$

$23 \mathrm{~b}$
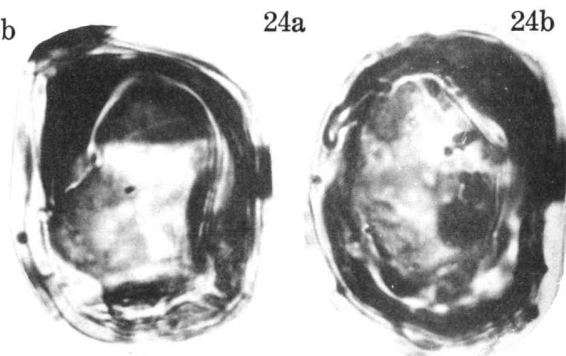

$25 a$

$25 \mathrm{~b}$

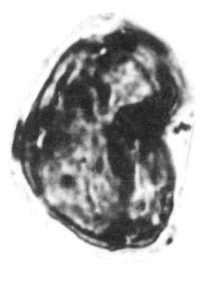

26
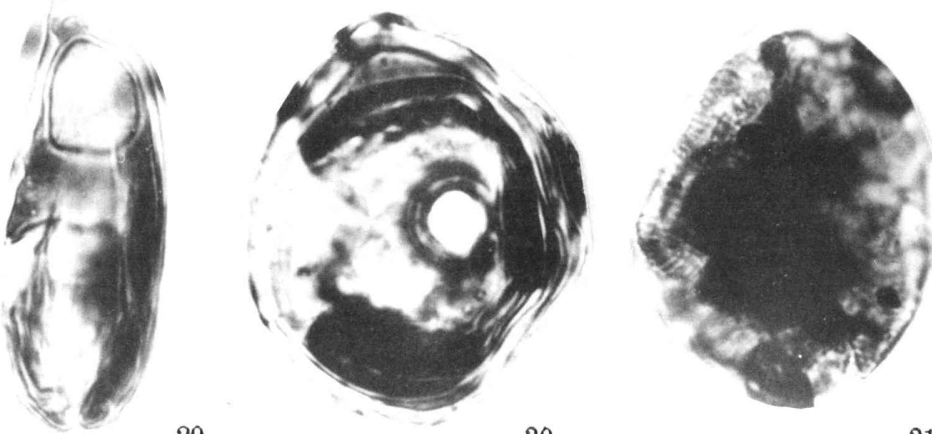

27

28

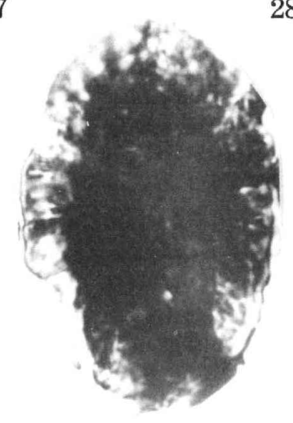

31

K. Takahashi : Sporen und Pollen der Hakobuchi-Schichtengruppe. 
Tafel 43 


\section{Erklärung zu Tafel 43}

(Noborikawa-Schichten bei der Talspere von Ooyubari; Fig. 1-22: ca. 833 fach vergr.; Fig. 23a: ca. 333 fach vergr.; Fig. 23b: ca. 533 fach vergr.)

Fig. 1. Microreticulatisporites sp. Präparat GK-V 3202.

Fig. 2, 3a, b. Subtriporopollenites kyushuensis TAKAHASHI

Fig. 2: Präparat GK-V 3201; Fig. 3a, b (cf.): Präparat GK-V 3204.

Fig. 4a, b. Betulaepollenites sp.

Präparat GK-V 3204.

Fig. 5. ? Triporopollenites sp.

Präparat GK-V 3204.

Fig. 6-8. Alnipollenites eminens (TAKAHASHI) n. comb.

Fig. 6: Präparat GK-V 3202; Fig. 7: Präparat GK-V 3204; Fig. 8:

Präparat GK-V 3203.

Fig. 9-11. Triporopollenites shimensis TAKahashI

Fig. 9 (cf.): Präparat GK-V 3203; Fig. 10, 11: Präparat GK-V 3204.

Fig. 12. Momipites constatus (TAKAHASHI) n. comb.

Präparat GK-V 3204.

Fig. 13. Ulmipollenites undulosus WoLFF

Präparat GK-V 3202.

Fig. 14. Ilexpollenites excellens (TAKAHASHI) n. comb. Präparat GK-V 3204.

Fig. 15. Ilexpollenites clavatus (TAKAHASHI) n. comb. Präparat GK-V 3202.

Fig. 16. Inaperturopollenites shikokuensis TAKAHASHI Präparat GK-V 3202.

Fig. 17-20. Inaperturopollenites pseudodubius TAKAHASHI

Fig. 17, 19, 20: Präparat GK-V 3203; Fig. 18: Präparat GK-V 3202.

Fig. 21. Tricolpopollenites minutiretiformis n. sp. Präparat GK-V 3203.

Fig. 22. Monocolpopollenites pfugii TAKAHASH Präparat GK-V 3201.

Fig. 23a, b. Piceaepollenites sp.

Präparat GK-V 3203. 

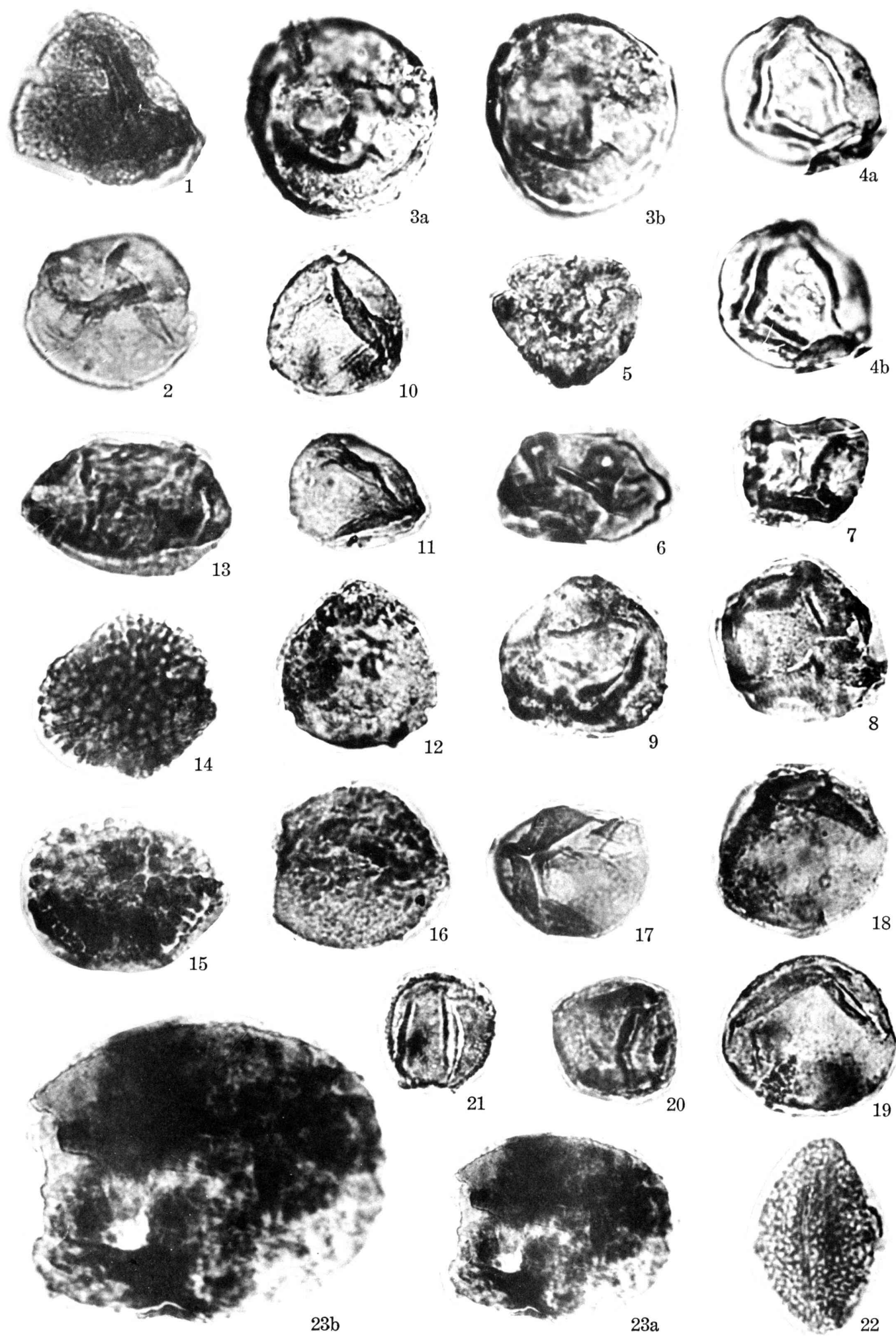

K. Takahashi: Sporen und Pollen der Hakobuchi-Schichtengruppe. 


\section{Tafel 44}




\section{Erklärung zu Tafel 44}

(Noborikawa-Schichten bei der Talspere von Ooyubari; ca. 833 fach vergr.)

Fig. 1-4. Tricolpopollenites rudis TAKAHASHI

Präparat GK-V 3202.

Fig. 5-10. Tricolpopollenites minutissmus n. sp.

Fig. 5, 7: Präparat GK-V 3203; Fig. 7: Holotypus; Fig. 6, 8-10:

Präparat GK-V 3202 .

Fig. 11-20. Tricolpopollenites ditis TAKAHASHI

Fig. 11, 15: Präparat GK-V 3203; Fig. 12: Präparat GK-V 3204; Fig. 13, 14, 16-20: Präparat GK-V 3202.

Fig. 21. Tricolpopollenites umiensis TAKAHASHI Präparat GK-V 3203.

Fig. 22,40. Tricolpopollenites vulgaris TAKAHASHI

Präparat GK-V 3202.

Fig. 23. Tricolpopollenites inamoenus TAKAHASHI Präparat GK-V 3203.

Fig. 24,25. Tricolporopollenites cf. cingulum (R. Рот.) Thомson \& Pflug subsp. fusus (R. Рот.) Tн. \& PF.

Fig. 24: Präparat GK-V 3204; Fig. 25: Präparat GK-V 3203.

Fig. 26-28. Tricolporopollenites castaneoides TAKAHASHI

Fig. 26: Präparat GK-V 3204; Fig. 27 (cf.): Präparat GK-V 3201； Fig. 28: Präparat GK-V 3202.

Fig. 29. Tricolporopollenites incertus TAKAHASHI Präparat GK-V 3203.

Fig. 30. Tricolpopollenites subasper TAKAHASHI Präparat GK-V 3203.

Fig. 31,32. Tricolpopollenites meinohamensis TAKAHASHI subsp. rotundus TAKAHASHI Präparat GK-V 3202.

Fig. 33. Tricolpopollenites sp. Präparat GK-V 3203.

Fig. 34-36. Tricolpopollenitss reticulatus TAKAHASHI Fig. 34, 35: Präparat GK-V 3203; Fig. 36 (cf.): Präparat GK-V 3202.

Fig. 37. Tricolpopollenites microreticulatus TAKAHASH Präparat GK-V 3203.

Fig. 38,39. Monocolpopollenites kyushuensis TAKAHASHI Präparat GK-V 3203.

Fig. 41. Rhoipites? hoshuyamaensis (TAKAHASHI) subsp. hoshuyamaensis n. emend. Präparat GK-V 3203.

Fig. 42. Ericipites sp. Präparat GK-V 3202.

Fig. 43,44. Periporopollenites asiaticus TAKAHASHI

Fig. 43: Präparat GK-V 3203; Fig. 44a, b: Präparat GK-V 3202. 

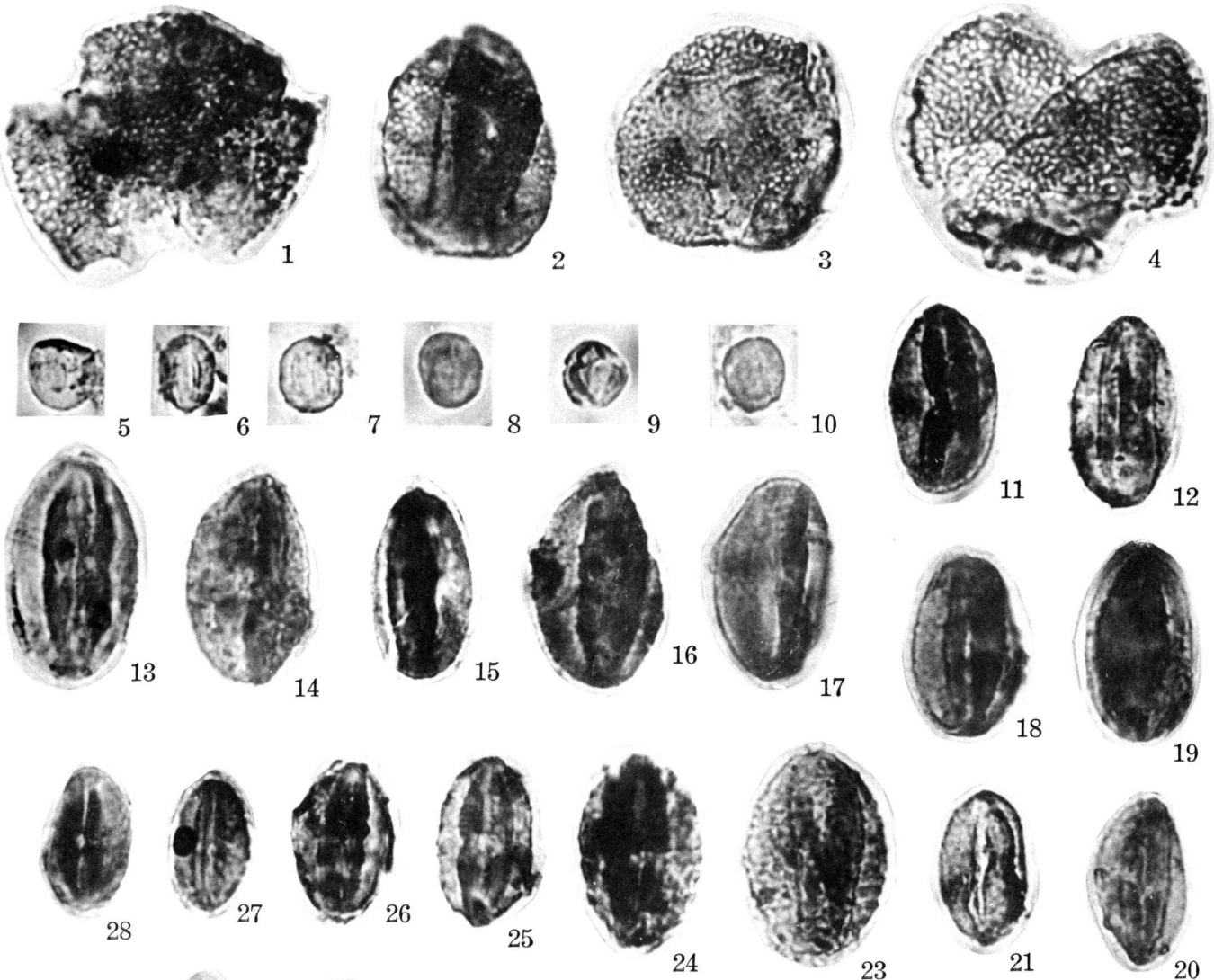

19
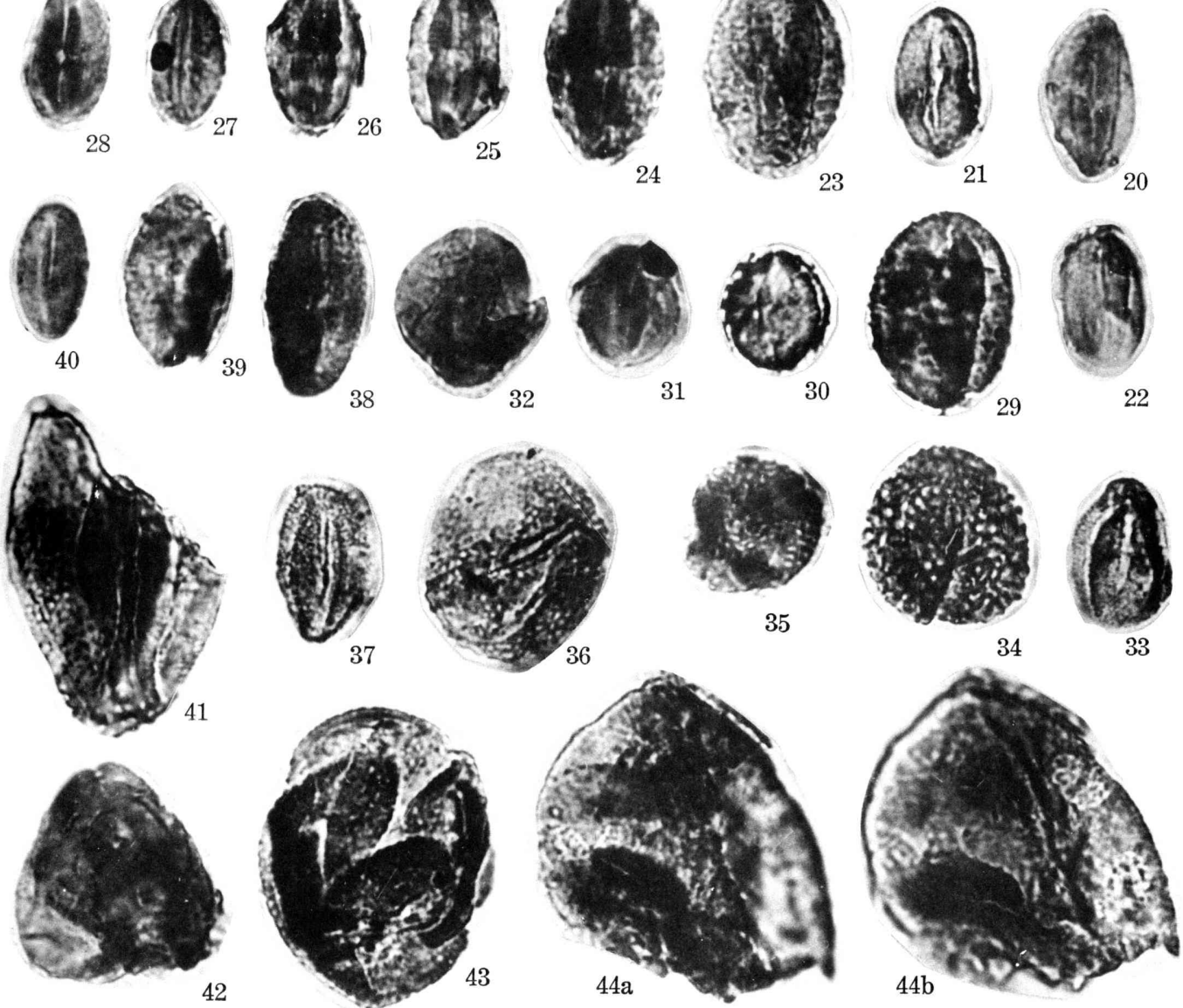

K. TaKahashi : Sporen und Pollen der Hakobuchi-Schichtengruppe. 